\title{
Halophytenvegetation im Chaco Boreal, Paraguay - Pflanzengeographie, Ökologie und Dynamik in Zeiten des Klima- und Landnutzungswandels
}

\author{
Dissertation \\ zur Erlangung des mathematisch-naturwissenschaftlichen Doktorgrades \\ "Doctor rerum naturalium" \\ der Georg-August-Universität Göttingen \\ im Promotionsprogramm Biologie \\ der Georg-August University School of Science (GAUSS)
}

vorgelegt von

M.Sc. Christian Vogt

aus Filadelfia, Paraguay

Göttingen, März 2014 


\section{Betreuungsausschuss}

Prof. Dr. Erwin Bergmeier, Abteilung Vegetationsanalyse und Phytodiversität, Albrecht-vonHaller-Institut für Pflanzenwissenschaften

Prof. Dr. Hermann Behling, Abteilung Palynologie und Klimadynamik, Albrecht-von-HallerInstitut für Pflanzenwissenschaften

\section{Mitglieder der Prüfungskommission}

Referent: Prof. Dr. Erwin Bergmeier, Abteilung Vegetationsanalyse und Phytodiversität, Albrecht-von-Haller-Institut für Pflanzenwissenschaften

Korreferent: Prof. Dr. Hermann Behling, Abteilung Palynologie und Klimadynamik, Albrecht-von-Haller Institut für Pflanzenwissenschaften

Prof. Dr. Ralph Mitlöhner, Abteilung Waldbau und Waldökologie der Tropen, BurckhardtInstitut, Fakultät für Forstwissenschaften und Waldökologie

Prof. Dr. Christoph Leuschner, Abteilung Pflanzenökologie und Ökosystemforschung, Albrecht-von-Haller-Institut für Pflanzenwissenschaften

Prof. Dr. Markus Hauck, Abteilung Pflanzenökologie und Ökosystemforschung, Albrechtvon-Haller-Institut für Pflanzenwissenschaften

Prof. Dr. Holger Kreft, Free Floater Nachwuchsgruppe - Biodiversität, Makroökologie und Biogeographie 
Meinen Eltern gewidmet 


\section{Inhaltsverzeichnis}

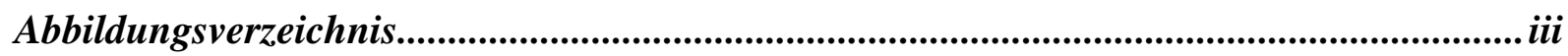

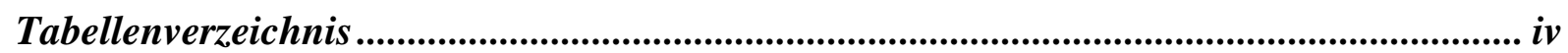

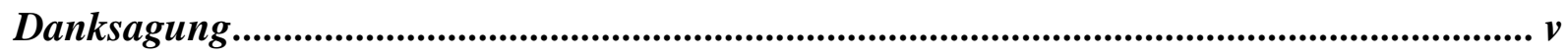

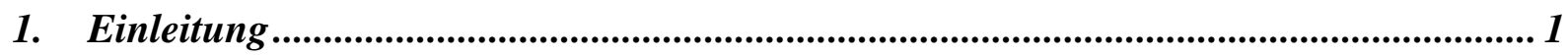

1.1. Problemstellung und Motivation.................................................................................... 1

1.2. Aktueller vegetationskundlicher Kenntnisstand im Untersuchungsgebiet ....................... 2

1.3. Zielsetzung und Aufbau der Arbeit .......................................................................................... 4

2. Naturräumliche Charakterisierung des Untersuchungsgebietes..................................... 5

2.1. Geografische Lage des Gran Chaco und des Chaco Boreal ........................................... 5

2.2. Geologische Verhältnisse und Böden im Chaco Boreal ........................................................... 5

2.3. Hydrologie ................................................................................................................................................. 6

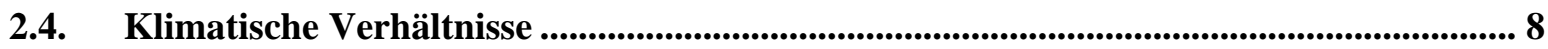

2.5. Engeres Untersuchungsgebiet ............................................................................................... 11

2.5.1. Untersuchungsorte im Departamento Alto Paraguay ............................................................ 13

2.5.2. Untersuchungsorte im Departamento Presidente Hayes ............................................................... 13

3. Phytogeographische Charakterisierung und Lebensformen-spektrum der Flora salzbeeinflusster Habitate im Chaco Boreal, Paraguay ........................................................ 16

3.1. Einleitung .......................................................................................................................................... 16

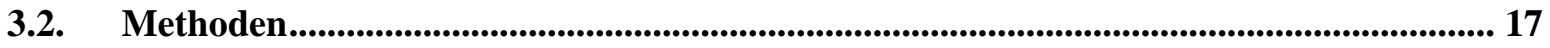

3.2.1. Checkliste der Gefäßpflanzen im paraguayischen Chaco ………………….......................... 17

3.2.2. Artenliste der salzbeeinflussten Habitate im paraguayischen Chaco ........................................... 17

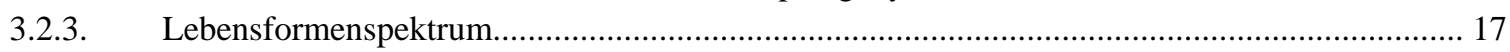

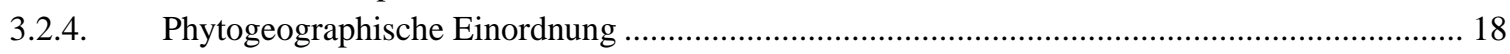

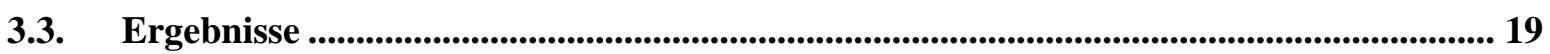

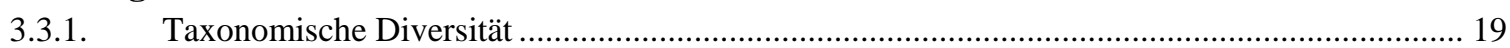

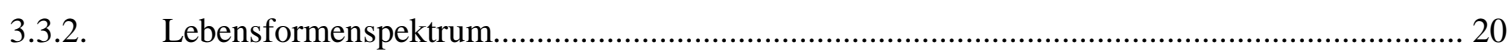

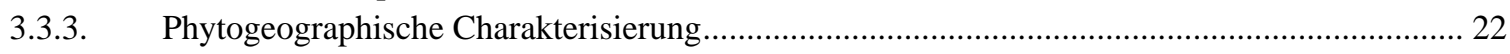

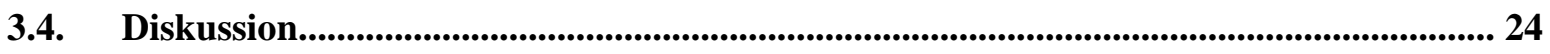

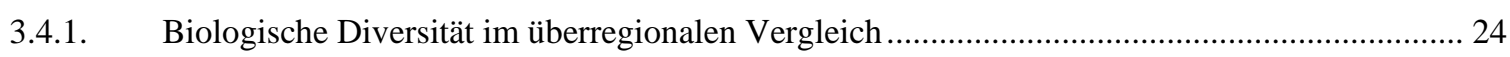

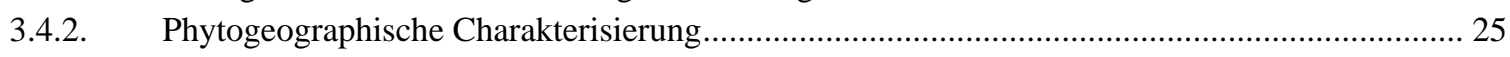

4. Klassifikation und ökologische Charakterisierung der Salz-marschen im Chaco von

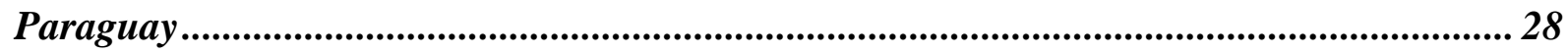

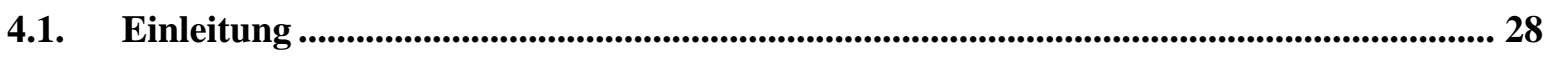

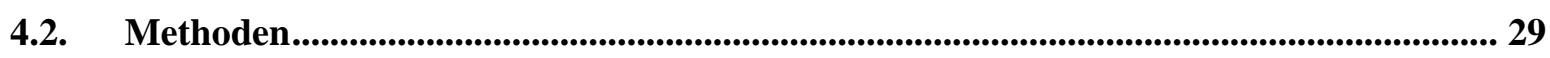

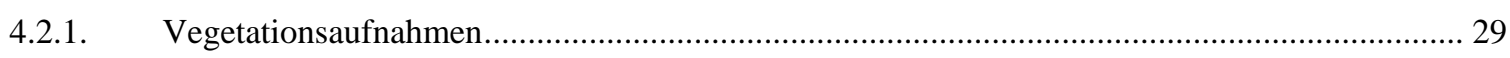

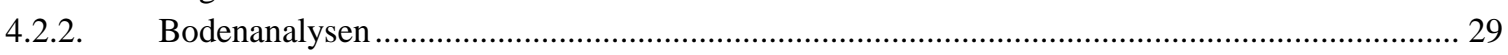

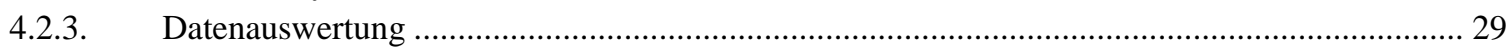

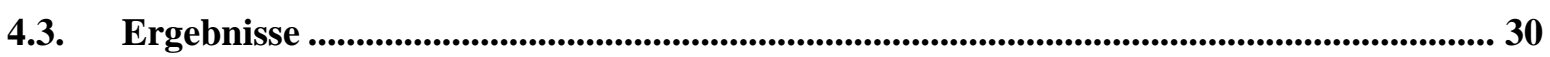

4.3.1. Klassifikation und Beschreibung der Gesellschaften......................................................... 30 
4.3.2. Ökologie und Dynamik der Salzmarschen............................................................... 37

4.4. Diskussion.........................................................................................................4 41

4.4.1. Halophytengesellschaften im überregionalen Vergleich....................................................... 41

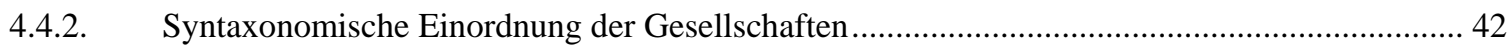

4.4.3. Ökologische Eigenschaften der Salzsteppen.................................................................. 43

5. Gesellschaften und strukturelle Diversität der Gehölzvegetation salzhaltiger Standorte im paraguayischen Chaco............................................................................................................... 46

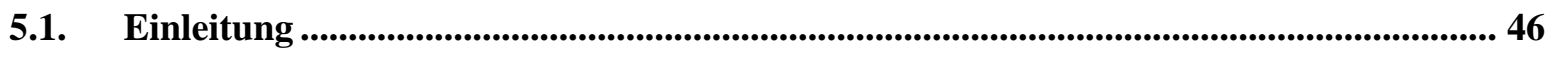

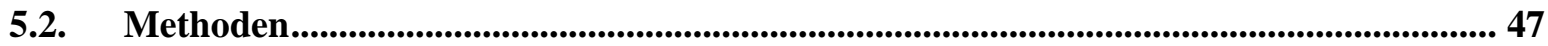

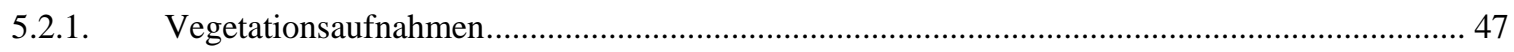

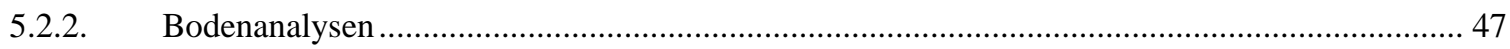

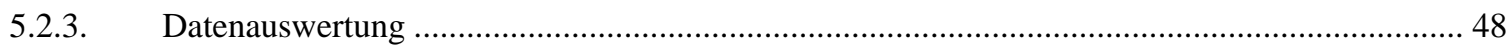

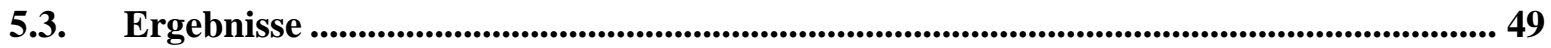

5.3.1. Klassifikation und Beschreibung der Gesellschaften.......................................................... 49

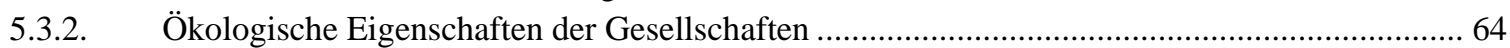

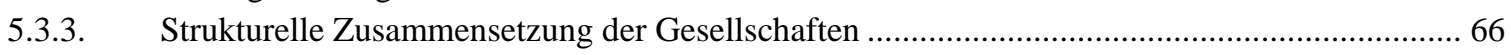

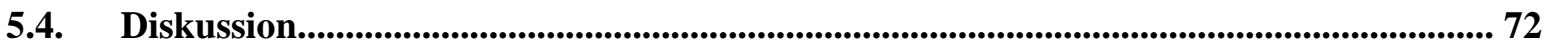

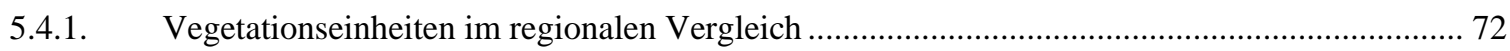

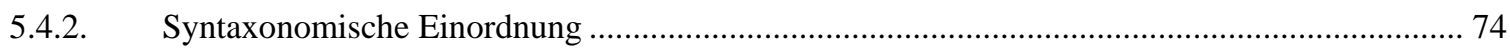

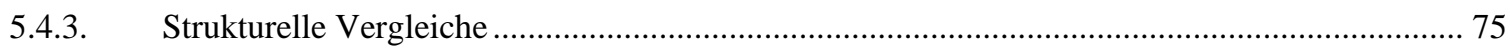

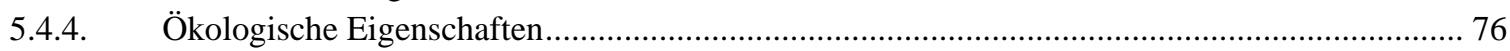

6. Abschließende Diskussion und Empfehlungsansätze ........................................................ 78

6.1. Diskussion der wichtigsten Forschungsergebnisse .................................................................. 78

6.1.1. Pflanzengeographische Stellung und Artenzusammensetzung der Vegetation salzbeeinflusster

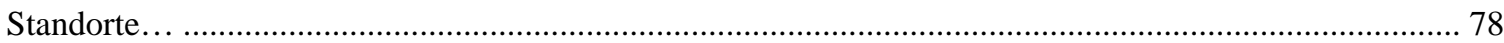

6.1.2. Pflanzensoziologische, ökologische und strukturelle Eigenschaften der salzbe-einflussten

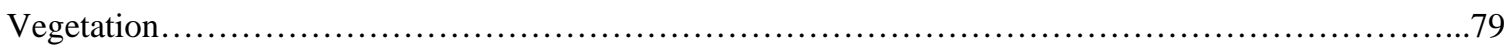

6.2. Offene Fragen und zukünftige Forschungsansätze ...................................................... 80

6.3. Entwicklungsprognosen der salzbeeinflussten Habitate im paraguayischen Chaco..... 81

6.3.1. Klimatische Veränderungen und ihre Auswirkung auf die Salzgebiete.................................... 81

6.3.2. Auswirkungen des immer stärker werdenden Landnutzungswandels..................................... 82

6.3.3. Alternative Nutzungsmethoden und notwendige Naturschutzmaßnahmen ............................. 83

7. Zusammenfassungen ............................................................................................................... 85

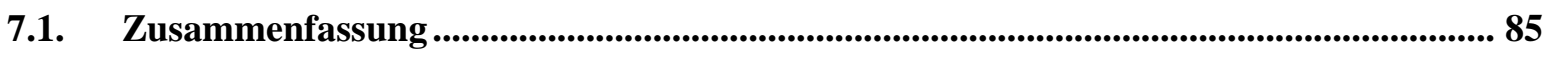

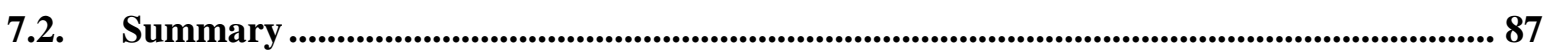

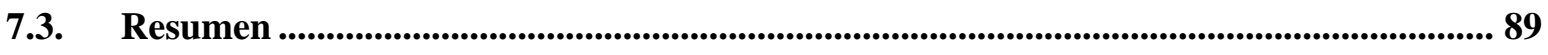

8. Abkürzungsverzeichnis und Glossar .................................................................................. 91

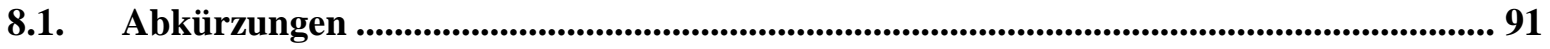

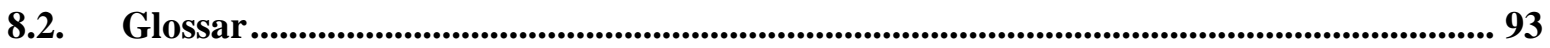

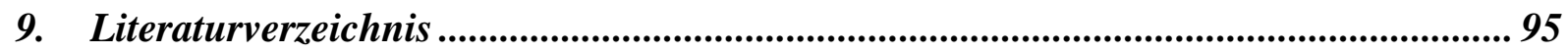

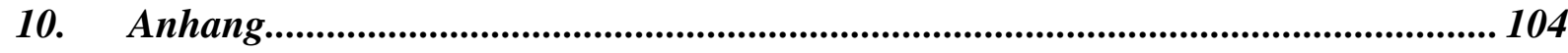




\section{Abbildungsverzeichnis}

Abb. 2-1: Der Gran Chaco Americano

Abb. 2-2: $\quad$ Landstreifen mit Bodenversalzungsphänomenen im Chaco Boreal............................. 7

Abb. 2-3: $\quad$ Mittlerer Jahresniederschlag im paraguayischen Chaco.......................................... 8

Abb. 2-4: Klimadiagramm für Loma Plata, Zentraler Chaco............................................. 9

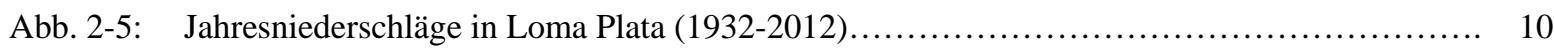

Abb. 2-6: $\quad$ Untersuchungsgebiet mit den einzelnen Untersuchungsorten............................... 12

Abb. 2-7: Salzhaltiges Flussbett des Riacho Curupayty............................................ 13

Abb. 2-8: Salzstandorte in der Umgebung der Laguna Ganso....................................... 14

Abb. 2-9: Riacho Yakaré Sur im Naturschutzreservat Campo Maria....................................................... 14

Abb. 2-10: Die Laguna Flamenco im Ramsargebiet Chaco Lodge..................................... 14

Abb. 3-1: Verteilung der Arten nach mittleren Jahresniederschlag im parag. Chaco und den Saladares... 20

Abb. 3-2: Lebensformenspektrum nach Raunkiaer im paraguayischen Chaco und im

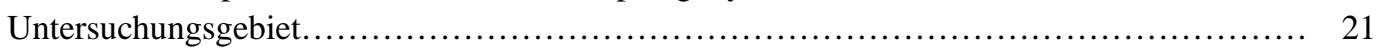

Abb. 3-3: Lebensformenspektrum der Arten im Untersuchungsgebiet................................ 21

Abb. 4-1: Ruppia maritima im Riacho Yakaré Sur............................................................................. 32

Abb. 4-2: Setaria geminata-Gesellschaft im Naturreservat Chaco Lodge.............................. 32

Abb. 4-3: DCA - Ordinationsdiagramm der krautigen Halophytengesellschaften......................... 33

Abb. 4-4: $\quad$ PCA - Ordinationsdiagramm des Leptochloa-Sarcocornia-Sesuvium Komplexes............... 33

Abb. 4-5: Vegetationsprofil am Ufer einer Salzlagune......................................... 34

Abb. 4-6: Sarcocornia ambigua-Gesellschaft im Naturreservat Chaco Lodge........................... 35

Abb. 4-7: Spartina spartinae-Gesellschaft am Riacho González................................ 35

Abb. 4-8: Vegetationsprofil einer typischen Salzpfanne..................................... 36

Abb. 4-9: Sesuvium portulacastrum-Sporobolus pyramidatus-Gesellschaft auf Sedimentaufschüttungen 36

Abb. 4-10: Heterostachys ritteriana-Gesellschaft in einem salzhaltigen und ausgetrockneten Flussbett.... 36

Abb. 4-11: Abgestorbene Heterostachys ritteriana-Population................................... 37

Abb. 4-12: Ordinationsdiagramm der häufigsten Salzmarsch-Gesellschaften in einer indirekten Gradientenanalyse mit den Bodenarten............................................... 38

Abb. 4-13: Ordinationsdiagramm der häufigsten Salzmarsch-Gesellschaften in einer indirekten Gradientenanalyse mit den chemischen Bodenparametern.............................. 38

Abb. 4-14: CCA - Ordinationsdiagramm der signifkanten Bodenparameter........................... 39

Abb. 4-15: Charakterisierung der Standorte einiger Halophyten.................................. 40

Abb. 5-1: Parkinsonia aculeata-Copernicia alba-Gesellschaft an der Laguna Rojas Silva................ 49

Abb. 5-2: DCA - Ordinationsdiagramm der Gehölzgesellschaften auf salzhaltigen und periodisch überfluteten Böden......................................................................... 50

Abb. 5-3: Prosopis ruscifolia-Sporobolus pyramidatus-Gesell-schaft am Riacho González............... 51

Abb. 5-4: Prosopis ruscifolia-Evolvulus convolvuloides-Gesell-schaft in der Reserva Yaguarete Porã $\quad 51$

Abb. 5-5: Lycium americanum-Cyclolepis genistoides-Gesell-schaft am Rand der Salzpfannen im Naturreservat Chaco Lodge............................................................... 52

Abb. 5-6: Prosopis ruscifolia-Tabebuia nodosa-Gesellschaft in der Reserva Natural Fortín Salazar...... 53

Abb. 5-7: PCA - Ordinationsdiagramm der Waldgesellschaften auf schwach bis mäßig dränierten

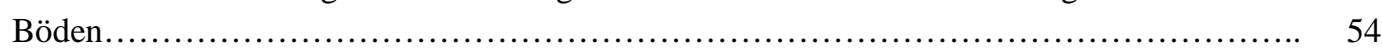

Abb. 5-8: Bulnesia sarmientoi-Gouinia paraguayensis-Gesellschaft................................ 55

Abb. 5-9: Schinopsis cornuta-Elionurus muticus-Gesellschaft im Ramsargebiet Laguna Rojas Silva...... 56

Abb. 5-10: Stark verarmte Schinopsis cornuta-Elionurus muticus-Gesellschaft bei Laguna Ganso........ 56

Abb. 5-11: Vegetationsprofil am Riacho Yakaré Sur...................................................... 57

Abb. 5-12: DCA - Ordinationsdiagramm der Gehölzgesellschaften 10 bis 18 in einer indirekten Gradientenanalyse mit den Bodenparametern.............................................. 64

Abb. 5-13: CCA - Ordinationsdiagramme der signifikanten Bodenparameter............................. 65 
Abb. 5-14: Absolute Frequenz der Höhen-Verteilung in den jeweiligen Gesellschaften....

\section{Tabellenverzeichnis}

Tab. 2-1: Chemische Eigenschaften des Oberflächenwassers einiger periodisch wasserführenden Flusssysteme im Untersuchungsgebiet. ..

Tab. 3-1: Einteilung des Niederschlagsgradienten im paraguayischen Chaco....................... 17

Tab. 3-2: Phytogeographische Elemente.................................................. 18

Tab. 3-3: Die wichtigsten Familien im paraguayischen Chaco und den salzbeeinflussten Standorten..... 19

Tab. 3-4: Floristischer Status der Arten im paraguayischen Chaco und den salzbeeinflussten Standorten ............................................................ 20

Tab. 3-5: Phytogeographische Einordnung der salzbeeinflussten Standorte und der Salzmarschen....... 22

Tab. 3-6: Lebensformenspektrum für die jeweiligen phytogeographischen Elemente 23

Tab. 3-7: Verteilung der phytogeographischen Elemente unter den am häufigsten vorkommenden Lebensformen............................................................ 23

Tab. 4-1: Synoptische Tabelle der krautigen Vegetationseinheiten mit Stetigkeitsangaben.............. 31

Tab. 5-1: Synoptische Tabelle der Gehölzvegetation mit Stetigkeitsangaben....................... 58

Tab. 5-2: Absolute Abundanz und absolute Dominanz der Gehölzarten mit einem BHD $\geq 10 \mathrm{~cm}$ in den jeweiligen Vegetationseinheiten............................................... 69

Tab. 5-3: Bedeutungswert-Index der häufigsten 20 Gehölzarten im Untersuchungsgebiet................ 71 


\section{Danksagung}

Mein größter Dank gebührt meinem Betreuer Prof. Dr. Erwin Bergmeier für sein persönliches Engagement in der Betreuung meiner Arbeit. Er hat mir in meinem Forschungsvorhaben Freiheit und Vertrauen geschenkt und mich in allen Phasen meiner Arbeit mit wertvollen Ratschlägen unterstützt.

Bedanken möchte ich mich bei meinem Zweitbetreuer Prof. Dr. Hermann Behling und bei Prof. Dr. Ralph Mitlöhner für Unterstützung und wertvolle Ratschläge. Außerdem danke ich Prof. i.R. Hartmut Dierschke, mit dem ich ein Büro teilen durfte, für interessante Hinweise und das angenehme Arbeitsklima.

Ein besonderer Dank gilt dem Deutschen Akademischen Austauschdienst (DAAD) für das Promotionsstipendium, welches mir den Aufenthalt und das Studium in Deutschland ermöglicht hat.

Des Weiteren will ich mich beim Hochschulrat der Universidad Nacional de Asunción und beim Fakultätssrat der Facultad de Ciencias Químicas bedanken, die für meinen Studienaufenthalt eine Beurlaubung von meinen Aufgaben als Wissenschaftlicher Mitarbeiter bewilligt haben.

An dieser Stelle sei den Dekanen der Facultad de Ciencias Químicas (FCQ), Prof. Dr. Andrés Amarilla, der Facultad de Ciencias Exactas y Naturales (FaCEN), Prof. Lic. Constantino Guefos und der Facultad de Ciencias Agrarias (FCA), Prof. Ing. Agr. Lorenzo Meza López für die vielseitige Unterstützung gedankt. Von der Facultad de Ciencias Químicas wurden die Kosten der Laboranalysen der Wasserproben übernommen. Die Facultad de Ciencias Exactas y Naturales stellte mir einen Geländewagen für mehrere Feldforschungsreisen ins Untersuchungsgebiet zur Verfügung und übernahm die gesamten Kosten dieser Reisen. Außerdem wurde ein großer Anteil der Kosten für die Laboranalysen der Bodenproben von der Facultad de Ciencias Agrarias übernommen.

Besonderer Dank gilt den Kollegen und Studenten, die mir bei den Geländearbeiten geholfen haben: Ana Rivarola, Marcelo Dujak, Maria Vera, Renata Araújo und Bernardo Cañiza.

Für den Zugang zu den verschiedenen Untersuchungsorten und Unterstützung während der Feldarbeiten möchte ich mich bei folgenden Personen bedanken: Ewald Friesen und Albert Unrau der Kooperative Chortizer Komitee (Reserva Natural Campo María und Laguna Capitán), Peter Dürksen und Wilfried Giesbrecht (Sitio Ramsar Chaco Lodge), Jakob Derksen sowie Heinz Theodor und Judith Friesen (Laguna Ganso), Heinz Löwen (Reserva Natural Riacho Florida), Marcelo Bastos Feraz (Reserva Natural Yaguareté Porá) und Roberto Eaton (Reserva Natural Fortín Salazar).

Ein großer Dank gilt ebenfalls Lic. Ana Maria Macedo der Red Paraguaya de Conservación en Tierras Privadas, für die Bereitstellung von Informationen und Kontaktaufnahmen mit den Landeigentümern. Bei Ing. For. Clara Echeverría bedanke ich mich für die Anfertigung der Satellitenkarten.

Des Weiteren bedanke ich mich bei meinen Kollegen vom Departamento de Botánica, insbesondere bei Gloria Delmás und Rosa Degen. Gloria war für mich die rechte Hand an der 
Universität in Paraguay und hat mich auf vieler Art und Weise unterstützt. Bei ihr möchte ich mich außerdem für die Profilzeichnungen bedanken.

Allen Mitarbeitern und Mitarbeiterinnen der Abteilung Vegetationanalyse und Phytodiversität danke ich herzlich! Die Kaffeepausen, Austauschrunden und netten Mensabesuche haben ganz sicher zum Gelingen dieser Arbeit beigetragen. In diesem Zusammenhang möchte ich mich ganz besonders bei Florian, Inga, Charlotte, Parastoo, Oliver, Naveed, Brigitte und Ute für die Unterstützung und Hilfestellungen bedanken.

Darüber hinaus möchte ich mich ganz besonders bei meinen Freunden und meinem Hauskreis für die gemeinsame Zeit, Gebete und mutmachende Worte bedanken. Auch meinem Onkel Adolfo gilt ein großer Dank für die vielseitige Unterstützung.

Nicht zuletzt danke ich ganz herzlich meinen Eltern, die mich in meinem Vorhaben immer unterstützt haben. Ohne ihre mentale, finanzielle und logistische Unterstützung wäre die Durchführung dieser Arbeit nicht möglich gewesen. 


\section{Einleitung}

\subsection{Problemstellung und Motivation}

Regionen mit hohen Salzgehalten in den Böden sind weltweit verbreitet und liegen einerseits in Meeresküstennähe und andererseits insbesondere in ariden Gebieten und Beckenlandschaften (Breckle 1976). Der Gran Chaco, das ausgedehnteste Trockengebiet in Südamerika, ist mit seinen besonderen geologischen und klimatischen Verhältnissen sehr anfällig für Boden- und Oberflächenversalzung. Die geringen Niederschläge einerseits und die hohen Verdunstungsraten andererseits verhindern die Auswaschung der leichtlöslichen Salze und fördern so die Entstehung von Salzböden. In Verbindung mit den klimatischen Verhältnissen werden in einigen Gebieten extreme Salzanreicherungen im Oberboden durch hohe Grundwasserstände und kapillar aufsteigendes, salzhaltiges Wasser begünstigt. Hinzu kommt das sehr geringe Gefälle der Chaco-Ebene, das den Oberflächenabfluss des Wassers und der Salze hemmt und bei Überschwemmungen zu lokalen Neuverteilungen der Salze führt.

Im Chaco Boreal von Paraguay sind laut Abrol et al. (1988) 20.000.000 ha der Böden salzbeeinflusst. In der Übergangszone von den regelmäßig überschwemmten Flächen des Bajo Chaco zum trockenen Zentralen Chaco kommen großflächige Salzstandorte vor, die man als „saladares“ oder „salitrales“ bezeichnet. Versalzungen, die in dieser Region auf natürliche Weise entstehen, treten hier im Bereich von Niederungen und Unterläufen periodisch wasserführender Flüsse auf und stehen in enger Verbindung zum hochanstehenden salzigen Grundwasser (Bender 1961). Diese Standorte werden von einer halophilen bis halotoleranten Vegetation besiedelt, die eine klare Zonierung entlang eines Salz- und Feuchtigkeitsgradienten von salzsteppenartiger Offenland-Vegetation über halophile Gebüschvegetation bis zum xeromorphen Trockenwald aufweist.

Die Vegetation der salzbeeinflussten Standorte im paraguayischen Chaco wurde bisher nur auf Formationsebene untersucht. Die floristische und strukturelle Zusammensetzung der Vegetation und die ökologische Anpassung einzelner Arten und der vorkommenden Pflanzengesellschaften an diese Standorte wurden bisher nicht untersucht. Vor dem Hintergrund massiver anthropogener Eingriffe und der sichtbaren Zunahme von Versalzungserscheinungen fehlten bisher Erkenntnisse bezüglich der ökologischen Aussagekraft und der Dynamik der Halophytenvegetation.

Der Mangel an ökologisch-pflanzensoziologischer Grundlagenforschung im paraguayischen Chaco und die daher fehlenden Erkenntnisse ökologischer Zusammenhänge an den Salzstandorten haben den Verfasser motiviert, diese Arbeit anzufertigen. Die Salzvegetation soll geobotanisch mit ihren floristischen, strukturellen und ökologischen Merkmalen charakterisiert und die Anpassung einzelner Arten und der vorkommenden Pflanzengesellschaften an den Standort herausgearbeitet werden. Eine Voraussetzung für dieses Vorhaben war eine kommentierte Liste der Pflanzenarten des paraguayischen Chaco, die der Verfasser in den letzten Jahren zusammengestellt und publiziert hat, die jedoch nicht in diese Dissertation integriert worden ist. 


\subsection{Aktueller vegetationskundlicher Kenntnisstand im Untersuchungsgebiet}

$\mathrm{Zu}$ Beginn dieser Arbeit lagen für die Salzstandorte im paraguayischen Chaco keine vegetationskundlichen Untersuchungen auf floristisch-pflanzensoziologischer Grundlage vor. Es standen nur grobe Vegetationsgliederungen der Formationen nach physiognomischökologischen Gesichtspunkten zur Verfügung. Insgesamt gibt es in Paraguay, und insbesondere im paraguayischen Chaco, bisher nur einige wenige pflanzensoziologische Untersuchungen nach der Methode von Braun-Blanquet (1964).

Pflanzengeographisch gehört der paraguayische Chaco zum nördlichen Teil des Gran Chaco, der von Cabrera (1970) und Cabrera \& Willink (1973) als Florenprovinz („Provincia Chaqueña“) innerhalb der Chaco-Florenregion („Dominio Chaqueño“) beschrieben wurde. Diese Florenprovinz entspricht auch der „Chacoan Province“ nach Takhtajan (1986) und den „Waldregionen des Chaco und seiner Randgebiete“ nach Hueck (1966).

López et al. (2006) untersuchte die Zusammenhänge zwischen den Trockenfloren Südamerikas und fand, dass der Chaco eine große floristische Ähnlichkeit mit der südlichen Trockenflora aufweist und zugleich eine gewisse Verbindung zwischen der südlichen und der nördlichen Flora zu bilden scheint.

In neueren biogeographischen Ansätzen von Prado \& Gibbs (1993) und Prado (2000) werden die Neotropischen Saisontrockenwälder („Dominio de los Bosques Secos Estacionales Neotropicales“), die als disjunkte Reliktvorkommen des Pleistozäns verstanden werden können, als separate Florenregion für die Neotropis genannt. Laut Prado (2000) und Oakley \& Prado (2011) sind diese Wälder in Paraguay hauptsächlich durch das „Misiones-Kerngebiet“ in Ostparaguay und teilweise auch im feuchten Chaco vertreten.

Untersuchungen zur Diversität der paraguayischen Dendroflora sind unter anderen von Bernardi (1984, 1985) und Spichiger et al. (1995) durchgeführt worden. Spichiger et al. (2004, 2005) haben die typischen Chacogehölze und -landschaftskomplexe anhand ihrer Verbreitung mit dem laubabwerfenden Paranáwaldes einerseits und den Neotropischen Saisontrockenwäldern andererseits verglichen und so biogeographisch charakterisiert.

Im Gran Chaco insgesamt wurden in einer ökoregionalen Auswertung (The Nature Conservancy et al. 2005) 53 terrestrische ökologische Systeme definiert, die in 44 ökologische Systemkomplexe eingeordnet wurden.

Laut Esser (1982) kann der paraguayische Chaco in drei Vegetationszonen geteilt werden: eine Überschwemmungsvegetation („Várzea“) in der Tal-Aue des Río Paraguay, einen regengrünen Wald und einen Trockenwald. Ramella \& Spichiger (1989) und Spichiger et. al. (1991) teilen die Vegetation entlang des Niederschlagsgradienten in ein WaldPalmensavannen-Mosaik im feuchten Ostchaco und einen Trockenwald im trockenen Westchaco. Aufgrund des Übergangscharakters von mesophiler zu xerophiler Vegetation und zur Abtrennung von den xerophilen Wäldern im trockenen Chaco verwenden Spichiger et al. (1991) für die Wälder im feuchten Chaco den Begriff „xeromesophil“. Laut Spichiger et. al. (2005) sind die Arten dieser xeromesophilen Wälder weniger frosttolerant als die des trockenen Chaco; jedoch sind mehr Generalisten vertreten. Die Benennung „xeromesophil“ wird von Mereles \& Degen (1998) und Mereles (2005) aufgegriffen und für die großräumige 
Landschaftseinteilung verwendet. So teilen diese Autoren den paraguayischen Chaco in einen xeromesophilen und einen xerophilen Landschaftskomplex.

In einem neuen pflanzengeographischen Ansatz unterscheiden Spichiger et al. (2006) im paraguayischen Chaco den trockenen Chaco und den trockenen Chaco mit psammophiler Fazies als homogene Landschaften und den feuchten Chaco als ökotonale Landschaft.

Auf Formationsebene wurde die Chacovegetation von Tortorelli (1966), Esser (1982), Spichiger et al. (1991), Huespe et al. (1991), Prado (1993b), Mereles \& Degen (1998) und Mereles (2005) untersucht. Spichiger et al. (1991) nennen sowohl für den trockenen als auch für den feuchten Chaco jeweils zonale, extrazonale und azonale Formationen. Von Huespe et al. (1991) werden zwölf Vegetationsformationen unterschieden und jeweils der Flächenanteil angegeben. In Anlehnung an Spichiger et al. (1991) und Mereles \& Degen (1998) klassifiziert Mereles (2005) für den paraguayischen Chaco 24 Vegetationsformationen und unterscheidet diese nach ihren hydrologischen und edaphischen Eigenschaften.

Einzelne Untersuchungen haben sich mit der Struktur und Artenzusammensetzung spezifischer Vegetationsformationen auseinandergesetzt. So untersuchten Pérez de Molas (1994) die Baumsavannen auf fossilen mit Sand gefüllten Flusssystemen im zentralen Chaco und Mereles (1998) das Wald-Palmensavannen-Mosaik im feuchten Ostchaco.

Ökologische Untersuchungen der Vegetation im paraguayischen Chaco sind auf einige wenige beschränkt. Von Mitlöhner (1990) wurde die Konkurrenz der Holzgewächse entlang des Niederschlagsgradienten untersucht. $\mathrm{Zu}$ den ersten Untersuchungen bezüglich der Beziehung zwischen Bodeneigenschaften und einzelner Gesellschaften entlang des Niederschlagsgradienten gehört die Arbeit von Navarro et al. (2011) im Biosphärenreservat im nördlichen Teil des Chaco.

Pflanzensoziologische Studien sind im paraguayischen Chaco bis heute ebenfalls nur einige wenige durchgeführt worden. Von Galán de Mera \& Navarro (1992) wurden erstmals die Wasserpflanzengesellschaften beschrieben und syntaxonomisch klassifiziert. Zur Gehölzvegetation insgesamt wurden einzelne Untersuchungen von Galán de Mera (2001) sowohl im trockenen als auch im feuchten Chaco und von Navarro (2005) und Navarro et al. (2006) entlang des Niederschlagsgradienten im Biosphärenreservat des nördlichen Teil des paraguayischen Chaco durchgeführt.

Im Gran Chaco insgesamt beschränken sich die pflanzensoziologischen Studien meist auf einzelne Landschaftsabschnitte und Gebiete oder weit verbreitete Vegetationsformationen. Der bolivianische Chaco ist diesbezüglich begünstigt da von Navarro \& Fuentes (1999) und Fuentes \& Navarro (2000) flächendeckende pflanzensoziologische Studien durchgeführt wurden. Außerdem wurde von Navarro \& Maldonado (2002) eine syntaxonomische Synopsis für den bolivianischen Chaco angefertigt. Im argentinischen Chaco gehen die ersten pflanzensoziologischen Studien zwar auf die Mitte des 20. Jahrhunderts zurück, doch ist bis heute keine flächendeckende Übersicht der vorkommenden Gesellschaften bekannt. So wurden von Cabido et al. (1991) die Wälder im Chaco Serrano und von Zak \& Cabido (2002) die Chacovegetation im Zentrum Argentiniens untersucht. Pflanzensoziologische Untersuchungen in salzbeeinflussten Gebieten im argentinischen Chaco wurden in den Salinas Grandes (Ragonese 1951, Ruiz Posse et al. 2007, Karlin et al. 2011) und in der Provinz Santa Fe (Lewis et al. 1990, Hilgert et al. 2003) durchgeführt. 


\subsection{Zielsetzung und Aufbau der Arbeit}

Das Ziel der vorliegenden Arbeit besteht darin, die Vegetation salzbeeinflusster Standorte im Chaco Boreal pflanzengeographisch, pflanzensoziologisch und ökologisch zu charakterisieren und ihre Stellung im südamerikanischen Kontext zu untersuchen. Im Mittelpunkt der Betrachtungen stehen die Artenzusammensetzung und Struktur der Vegetation entlang eines Gradienten von stark salzhaltigen bis zu mäßig salzbeeinflussten Standorten und die daraus resultierende vegetationsökologische Charakterisierung. Des Weiteren soll diese Arbeit eine Entwicklungsprognose der Binnenlandsalzstellen und ihrer Vegetation unter aktuellen Klimawandel- und Landnutzungsszenarien geben und so eine Hilfestellung für den angewandten Naturschutz leisten.

Die Arbeit besteht aus drei Ergebniskapiteln, die in unterschiedlichen Ansätzen die Vegetation der salzbeeinflussten Standorte im Chaco Boreal charakterisieren und beschreiben:

> Phytogeographische Charakterisierung und Lebensformenspektrum (Kapitel 3): Ziel dieses Kapitels ist es die Artenzusammensetzung der untersuchten Salzstandorte pflanzengeographisch zu charakterisieren und mit den im paraguayischen Chaco vorkommenden Arten bezüglich des Lebensformenspektrums und der geographischen Verbreitung zu vergleichen. Grundlage für diese Vergleiche ist die vorab vom Verfasser angefertigte Checkliste der Gefäßpflanzen im paraguayischen Chaco (Vogt 2011, 2012, 2013).

> Klassifikation und ökologische Charakterisierung der Salzmarschen (Kapitel 4): In diesem Kapitel wird die in den Salzpfannen und angrenzenden salzhaltigen Randbereichen dominierende Offenland-Vegetation mit halophilen Zwergsträuchern und Kräutern pflanzensoziologisch und ökologisch charakterisiert.

> Gesellschaften und strukturelle Diversität der Gehölzvegetation (Kapitel 5): Das Ziel dieses Kapitels besteht darin, die im Untersuchungsgebiet dominierende salzbeeinflusste Gehölzvegetation anhand pflanzensoziologischer, ökologischer und struktureller Untersuchungen zu klassifizieren und die Dynamik ansatzweise zu beschreiben.

Abschließend werden im 6. Kapitel themenübergreifende Diskussionspunkte aufgegriffen und einige Schlussfolgerungen gezogen. Die Empfehlungsansätze und Entwicklungsprognosen sollen als Denkanstöße für zukünftige Nutzungs- und Naturschutzmaßnahmen sowie für weitere Untersuchungen verstanden werden. 


\section{Naturräumliche Charakterisierung des Untersuchungsgebietes}

\subsection{Geografische Lage des Gran Chaco und des Chaco Boreal}

Der Gran Chaco als geographischer Großraum (Abb. 2-1) mit $1.066 .000 \mathrm{~km}^{2}$ liegt im Zentrum des südamerikanischen Kontinents und ist auf vier Länder verteilt: Argentinien (62,2\%), Paraguay (25,4\%), Bolivien (11,6\%) und Brasilien (0,8\%). Mit einer Nord-SüdAusdehnung von tropischen Breitengraden $\left(18^{\circ} \mathrm{S}\right)$ bis $\mathrm{zu}$ subtropischen Gegenden $\left(31^{\circ} \mathrm{S}\right)$ weist diese Ökoregion eine breite Vielfalt von Naturräumen auf (The Nature Conservancy et al. 2005). Im Westen wird der Gran Chaco von den Anden, im Osten vom Río Paraguay, im Norden vom bolivianischen ChiquitosBergland und im Süden vom Río Salado (Argentinien) begrenzt.

Paraguay wird durch den Río Paraguay in eine östliche und eine westliche Region geteilt. Mit $246.925 \mathrm{~km}^{2}$ hat die westliche Region (Chaco) eine Flächenanteil von $61 \%$ des Territoriums von Paraguay. Die Bevölkerungs-dichte beträgt nur 0,7 Einwohnern $/ \mathrm{km}^{2} \quad$ (DGEEC 2013). Politisch-administrativ ist der paraguayische Chaco in drei

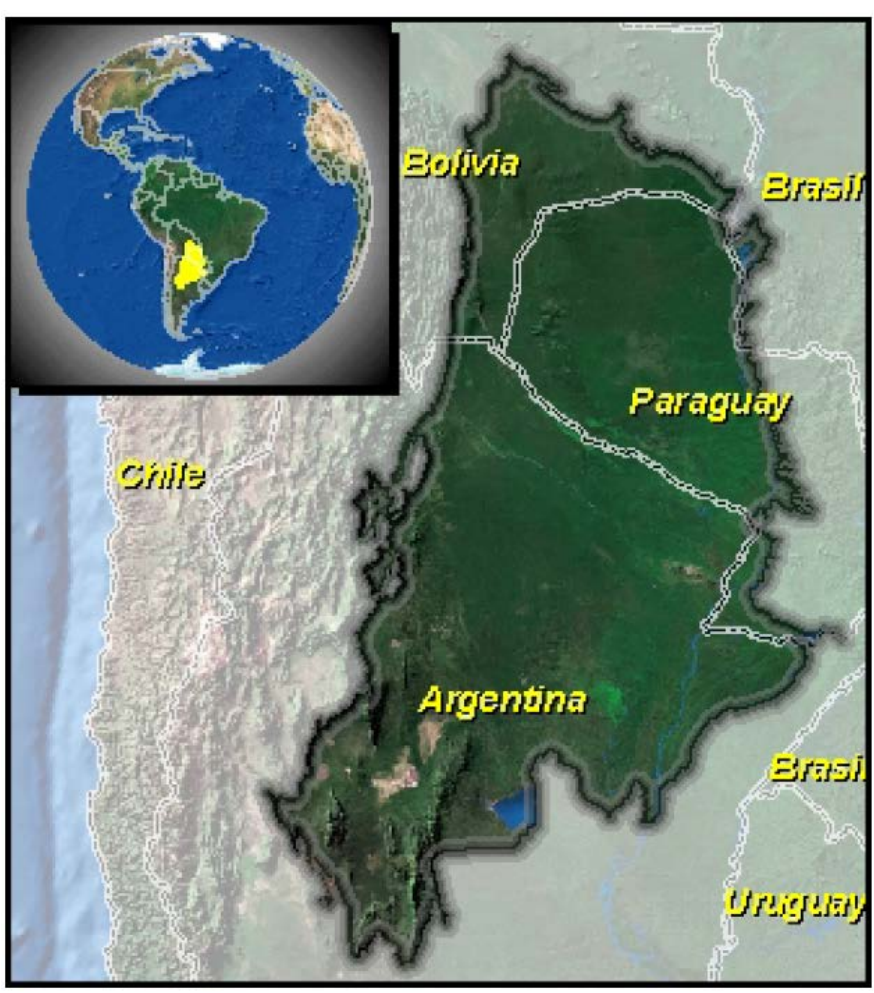

Abb. 2-1: Der Gran Chaco Americano. (entnommen aus The Nature Conservancy et al. 2005) Departamentos geteilt: Alto Paraguay, Boquerón und Presidente Hayes.

\subsection{Geologische Verhältnisse und Böden im Chaco Boreal}

Das Chaco-Becken ist ein epikontinentaler Trog, der zwischen dem Brasilianischen Schild und den Kordilleren liegt und regional-tektonisch das „Vorland“ der gefalteten Ketten der bolivianischen und argentinischen Anden darstellt (Putzer 1962).

Der tiefere Untergrund des Chaco-Trogs besteht aus über $2000 \mathrm{~m}$ mächtigen marinen Sedimenten in geosynklinaler Fazies, die im Silur und Devon (Paläozoikum) im Chaco abgelagert worden sind. Das marine Paläozoikum wird von 500 m bis 2500 m mächtigen rötlichen kontinentalen Sedimenten, den sogenannten Red Beds, überlagert. Sie werden mit Vorbehalt dem großen Zeitabschnitt vom Ober-Karbon bis zur Trias zugerechnet. Über den Red Beds folgen bis 500 m mächtige junge, halb- bis nichtverfestigte festländische Gesteine 
des Känozoikum (Tertiär und Quartiär), die das nach Korngrößen geordnete Abtragsmaterial aus den Anden darstellen (Putzer 1962).

Die heutige Ebene des Chacos dürfte ihren Ursprung im späten Tertiär mit der Heraushebung der Anden sowie einer intensiven Abtragung des Materials in das östliche Vorland, den Chaco, haben. Im westlichen gebirgsnahen Teil befindet sich das gröbere Abtragsmaterial aus den Anden, während Feinsand, Löss und Ton entsprechend der Transportenergie der Flüsse und des Windes weiter über die Ebene verteilt sind (Bender 1961).

Die steinlose Ebene des paraguayischen Chaco steigt vom Ufer des Río Paraguay - im Norden bei 120 m, im Süden bei 55 m über dem Meeresspiegel beginnend - nach Westen an und erreicht an der bolivianischen Grenze eine Höhe von über 300 m.

Die Böden im Chaco Boreal sind im allgemeinen sehr jung und tiefgründig durchwurzelbar. Die Bodenentwicklung hängt sehr stark vom Ausgangsmaterial und von den Niederschlägen bzw. von der Bodendurchfeuchtung ab (Kruck et al. 1998). Die Bodenausgangsgesteine sind schluffige Tone sowie tonige bis schluffige Feinsande fluviatilen und äolischen Ursprungs (Lüders 1962).

Während im semiariden Westen des Chacos die Böden nur sehr schwach entwickelt sind (Arenosole, Regosole), überwiegen im östlichen Teil tonreiche und staunasse Böden (Gleye, Vertisole) (Kruck et al. 1998, González Erico 2007). Im zentralen Chaco sind die Böden grundwasserferner Standorte überwiegend den Luvisolen und Cambisolen zuzuordnen. Sie zeichnen sich durch eine schluffig-tonige Textur, einen tonreichen B-Horizont, eine neutrale bis schwach alkalische Bodenreaktion sowie eine hohe Basensättigung aus. Grundwassernahe Standorte dagegen zeichnen sich durch hohe Salzgehalte im Bodenkörper aus. Diese Böden lassen sich den Salz-Natrium-Böden (Solonetz, Solonchak) zuordnen, die neben hohen Salzgehalten auch eine hohe Natriumsättigung am Austauscherkomplex aufweisen. Die tonigschluffige Textur, die im Tiefenverlauf zunehmend toniger wird, sowie die geringe Ausbildung an Grobporen verhindern eine ausreichende Dränage bei starken Niederschlägen, so dass es zu Überstauungen des Oberbodens kommen kann (Wiebe 2003).

\subsection{Hydrologie}

Die Landschaften der Chaco-Ebene sind nach Ramella \& Spichiger (1989) Ausdruck des Wassers, das sowohl als klimatischer als auch als morphologischer Faktor angesehen werden kann. Nach ihren Ursprüngen können drei verschiedene oberirdische hydrographische Systeme im Chaco unterschieden werden:

a) Andiner Ursprung: Die drei in den Anden entspringenden Flüsse, die große Teile des andinen Regenwassers in die Chaco-Ebene bringen, sind der Río Bermejo (Argentinien), Río Pilcomayo (Bolivien, Argentinien und Paraguay) und Río Parapití (Bolivien). Nur die ersten beiden durchqueren den Gran Chaco von Westen nach Osten. Für die Landschaftsbildung im Chaco Boreal sind hauptsächlich der Río Pilcomayo und der Río Parapití verantwortlich.

b) Ursprung im Pantanal: Obwohl der Río Paraguay nicht als Chaco-Fluss bezeichnet werden kann, bildet er die östliche Grenze der Florenprovinz und nimmt das Wasser der hydrographischen Chaco-Systeme auf. 
c) Ursprung im Chaco: Bestimmend für die hydrographischen Systeme, die im Chaco entspringen, sind die Niederschläge in den Sommermonaten. Das geringe Relief der Chaco-Ebene bedingt, dass weite Gebiete nicht oder nur wenig entwässert werden. Im trockenen Chaco kommen zahlreiche fossile Flusstäler vor, die zugeschüttet und aus der Luft als trockengelegte oder stumpfe Flussmäander (endorheische Flusssysteme) erkennbar sind. Im feuchten Chaco trifft man überwiegend langgestreckte Lagunen und ausgedehnte Sümpfe als Relikte einstiger Talauen sowie aktive und exorheische Flüsse die den Río Paraguay erreichen (wie z.B. Río Montelindo, Río Verde, Río Confuso, Río Negro). Wegen des fehlenden Gefälles dieser Region sind vor allem im zentral-östlichen Chaco zahlreiche abflusslose Mäander und Lagunen anzutreffen (Ramella \& Spichiger 1989).

Laut Junker \& von Hoyer (1998) ist der Chaco geologisch ein riesiger Schwemmfächer von Flusssedimenten (Alluvionen), die aus den Anden in das südöstliche Vorland transportiert wurden. Hydrogeologisch handelt es sich also um eine stark alternierende Wechselfolge von Wasserleitern und Wasserstauern unterschiedlicher Qualität. Das Gebiet des zentralen und westlichen Chaco bis zum Río Pilcomayo umfasst das Paläodelta des Pilcomayo. Feinsand, Schluff und Ton in lateralem wie vertikalem Wechsel wurden hier vom Fluss abgelagert. Der flachen Neigung der alten Deltafläche folgend fließt das Grundwasser langsam von West nach Ost mit Fließgeschwindigkeiten zwischen 0,6 und 1,8 m/Jahr.

Ein interessantes Problem stellt der Salzgehalt der oberen Schichten der Chaco-Sedimente dar. Salinare Ablagerungen sind in der Schichtenfolge bisher nicht bekannt, doch wurde nachgewiesen, dass alles tiefere Grundwasser versalzen ist, während im zentralen und westlichen Chaco Boreal das oberflächennahe Grundwasser auch Süßwasser-Linsen enthält (Putzer 1962). Geoelektrische Messungen (Bender 1961) zeigten große Unterschiede in den Salzgehalten des Grundwassers, so dass davon ausgegangen wird, dass es im ersten Grundwasser-Stockwerk keinen zusammenhängenden Grundwasserstrom gibt, sondern sich um Senken, Taschen und rinnenförmige Vertiefungen handelt, die Reste eines Entwässerungssystems darstellen. Unterhalb der ersten Sohlschicht befindet sich ein geschlossener zweiter Grundwasserkörper, der hochgradig versalzen ist (bis $60 \mathrm{mS} / \mathrm{cm}$ ). Im zentral-östlichen Chaco wird aufgrund der abnehmenden Mächtigkeit des Oberbodens das zweite Grundwasser-Stockwerk örtlich in Niederungen angeschnitten (Wiebe 2003). In diesem ungefähr $75 \mathrm{~km}$ breiten und $500 \mathrm{~km}$ langen Landstreifen kommen häufig Bodenversalzungsphänomene vor (Wiens 1998, Glatzle et al. 2000) (Abb. 2-2).

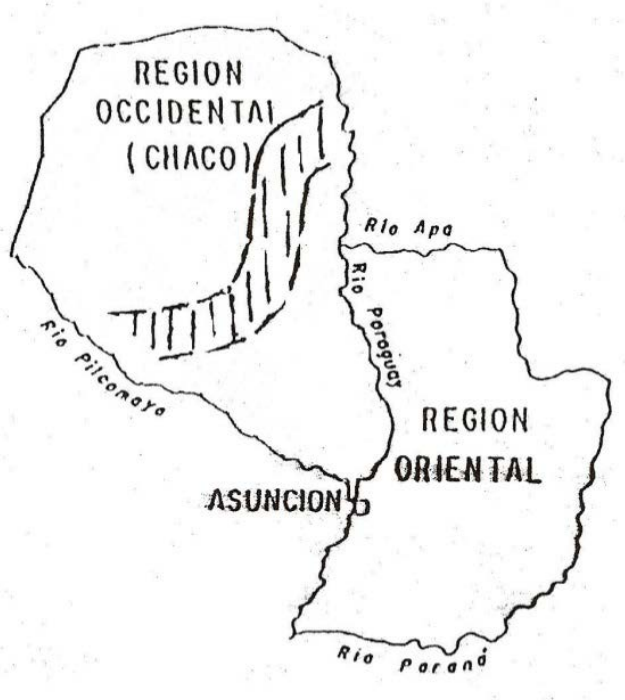

Abb. 2-2: Landstreifen mit Bodenversalzungsphänomenen im Chaco Boreal. (entnommen aus Wiens 1998). 


\subsection{Klimatische Verhältnisse}

Durch seine Lage am südlichen Wendekreis herrscht im Chaco Boreal tropisch-subtropisches Übergangsklima vor. Durch die Barriere der Anden ist Zentral-Südamerika vom pazifischen Einfluss abgeriegelt. Da die äquatoriale und subantarktische Zone durch keine Klimaschranken getrennt sind, ist das Klima durch jederzeit mögliche Wetterstürze wechselhaft (Esser 1982).

Laut Grassi et al. (2005) herrscht nach der Klimaklassifikation von Köppen im östlichen Chaco ein tropisches Savannenklima und im zentral-westlichen Teil ein tropisches Steppenklima.

Wetterbestimmend für den Chaco sind wechselnde Windrichtungen. Die im Sommer vorherrschenden Nordwinde bringen warme Luftmassen und sind häufig mit Tiefdruckgebieten verbunden. Südwinde bringen im Gegensatz dazu kalte und trockene Luftmassen und sind meist mit Hochdruckgebieten assoziiert. Wetterwirksame Ost- und Westwinde treten kaum in Erscheinung. Winde mit einer Geschwindigkeit von mehr als 22 km/h wehen nach Wiebe (2003) zu 80\% aus nördlichen und nordöstlichen Richtungen.

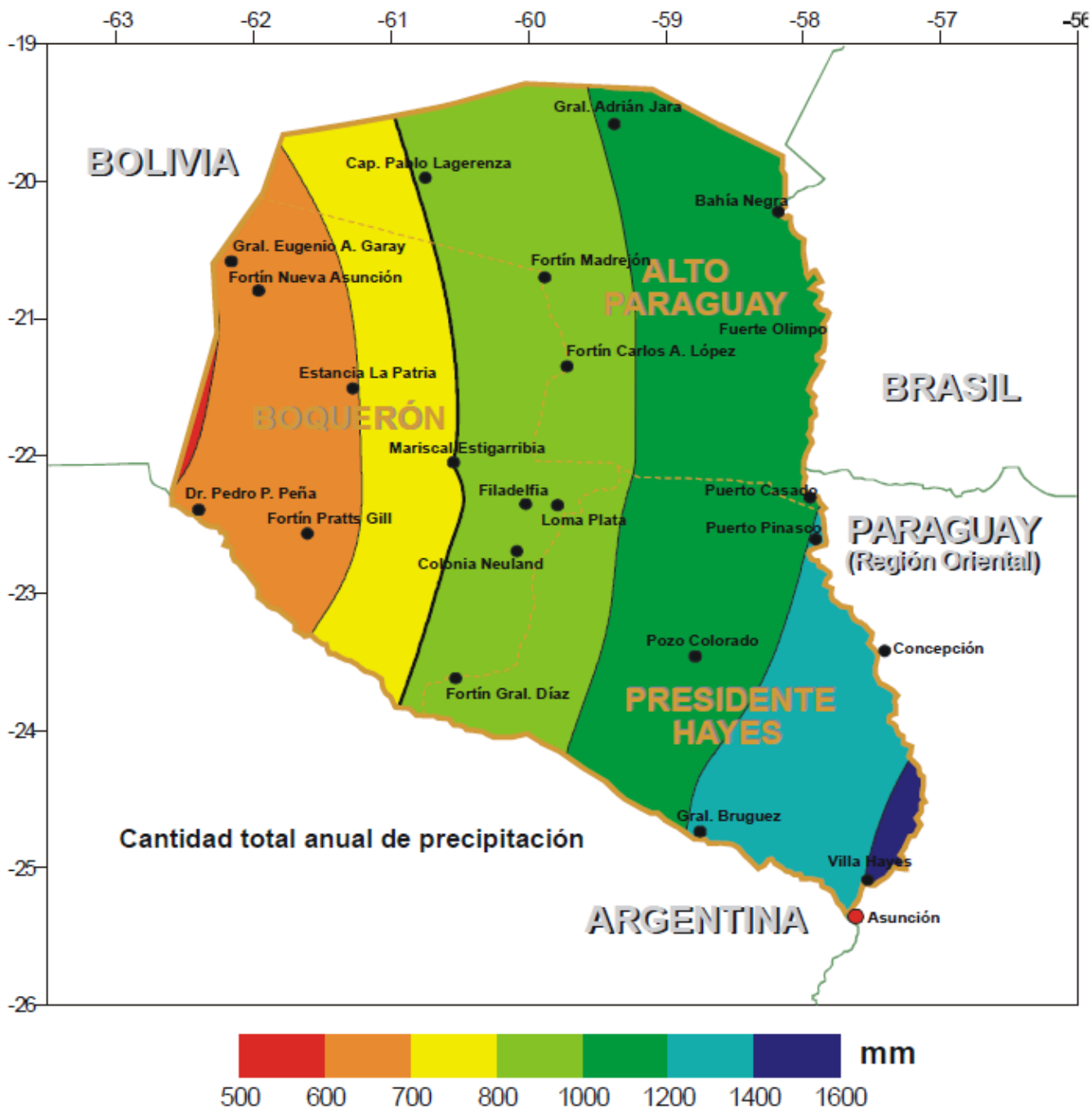

Abb. 2-3: Mittlerer Jahresniederschlag im paraguayischen Chaco (entnommen aus Grassi et al. 2005). 
Auffälligstes Merkmal des Klimas im Chaco ist ein ausgeprägter Feuchtegradient vom Río Paraguay (1400 mm mittlerer Jahresniederschlag) zum westlichen semiariden Chaco (unter $500 \mathrm{~mm}$ mittlerer Jahresniederschlag)(Abb. 2-3). Nicht nur die Niederschlagssummen nehmen ab, sondern auch die Anzahl der Trockenmonate nimmt zu. Während in der Talebene des Río Paraguay ein wirklicher Trockenmonat fehlt, hat der Zentrale Chaco bereits vier bis fünf, der westliche semiaride Chaco sogar zehn Trockenmonate (Gorham 1973, Esser 1984).

Zum Klima im Chaco gehören die extrem heißen Sommer und die gemäßigten Winter. Es gibt eine bedeutende Amplitude zwischen der mittleren Tagestemperatur im Januar $\left(28,5{ }^{\circ} \mathrm{C}\right)$ und der mittleren Tagestemperatur im Juli $\left(18,9{ }^{\circ} \mathrm{C}\right)$. Im Frühling und Sommer kann es zu Höchsttemperaturen von $46^{\circ} \mathrm{C}$ kommen und in den Wintermonaten, besonders im Juli, können Tiefsttemperaturen von $-7^{\circ} \mathrm{C}$ gemessen werden. Die Amplitude dieser Extremtemperaturen überschreitet $50^{\circ} \mathrm{C}$ (Grassi et al. 2005). Das Klimadiagramm für Loma Plata im zentralen Chaco Boreal (Abb. 2-4) zeigt die mittlere Monatstemperatur und die mittleren Monatsniederschläge und legt eine 4-5 monatige Dürrezeit nahe. Die kühleren Monate (Mai bis September) stellen eine niederschlagsarme bis niederschlagsfreie Periode dar, während in den Sommermonaten (Oktober bis April) kurzandauernde heftige Niederschläge fast den gesamten Jahresniederschlag ausmachen.

Wie die Niederschlagsmengen der letzen 81 Jahre in Loma Plata (Abb. 2-5) zeigen, gibt es eindeutige Variationen, die auf klimatische Zyklen hindeuten. Besonders hohe Jahresniederschläge, wie z. B. in den Jahren 1982/83, stehen meist mit dem El NiñoPhänomen in Verbindung. Es kann außerdem eine tendenzielle Niederschlagszunahme beobachtet werden. Die steigende Tendenz in den Niederschlägen wurde nach Grassi et al. (2005) auch für andere Orte im Chaco beobachtet.

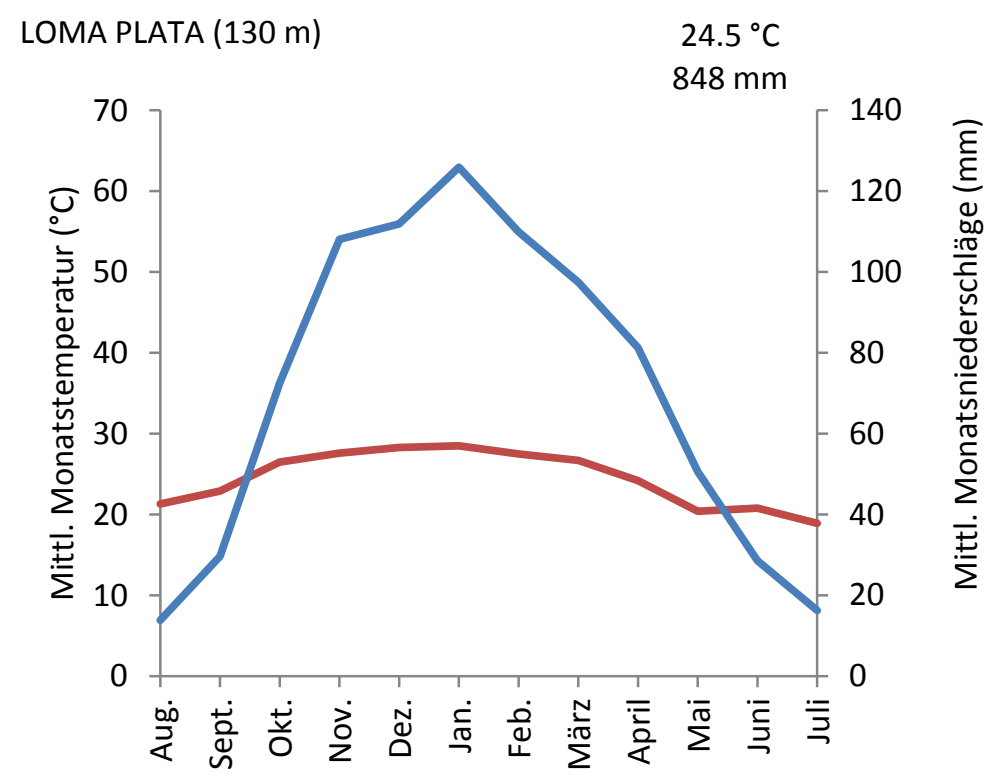

\footnotetext{
Abb. 2-4: Klimadiagramm (nach Walter 1955) für Loma Plata, Zentraler Chaco. Gezeigt werden die mittleren Monatsniederschlage (1932-2012) und die mittlere Monatstemperatur (1982-1991). Die Daten wurden vom Centro de Formación Profesional und von Herrn Johann J. Sawatzky aufgenommen.
} 


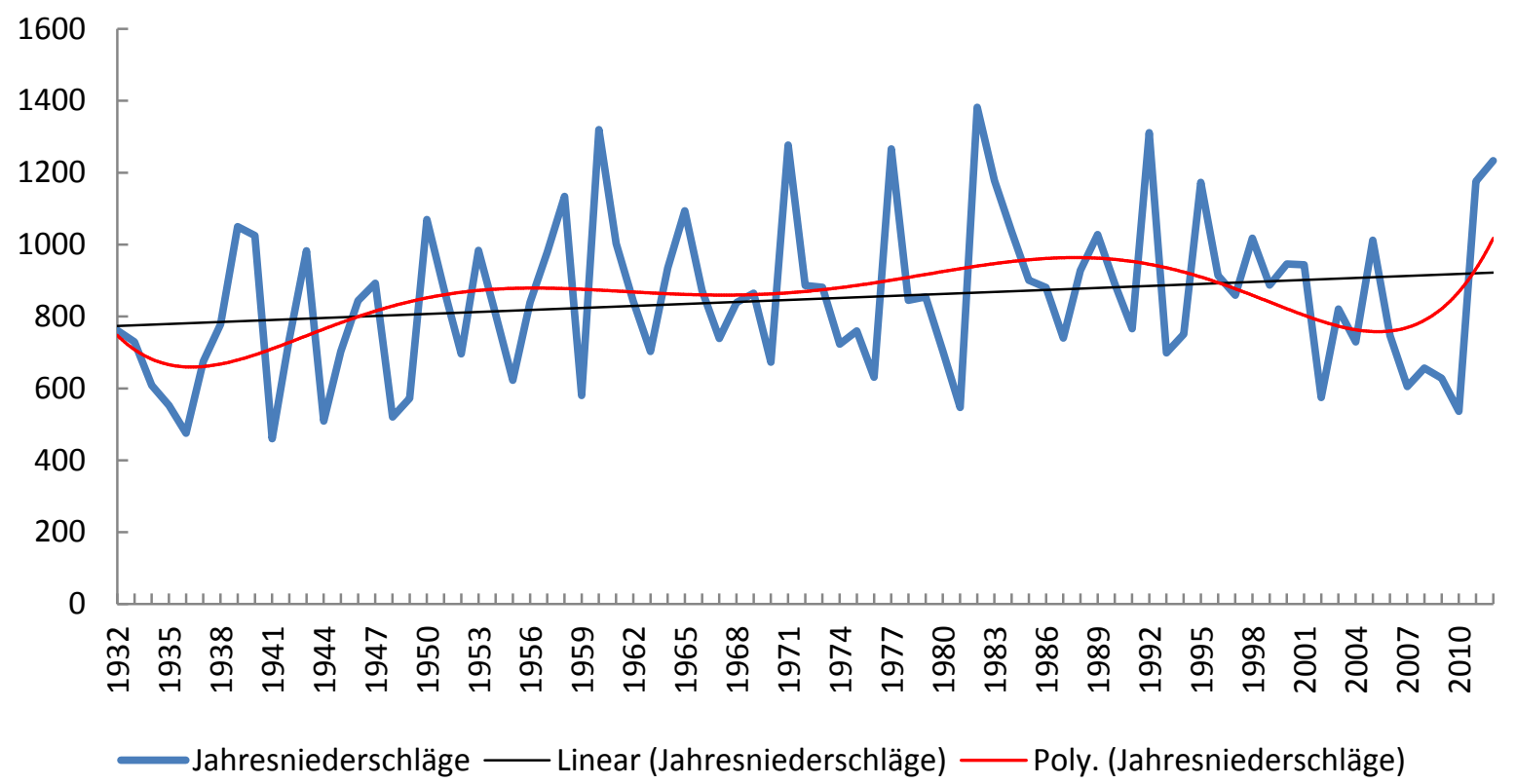

Abb. 2-5: Jahresniederschläge in Loma Plata (1932-2012) mit Niederschlagstendenzen (linear und polygonisch)

Die potentielle Evapotranspiration liegt nach Harder et al. (2004) im zentralen Chaco um $1700 \mathrm{~mm}$. Nach der Klimaklassifikation von Thornthwaite, die auf der potentiellen Evapotranspiration und den Niederschlägen basiert, kann das Klima im zentral-östlichen Chaco als trocken subhumid und im zentral-westlichen Teil als semiarid eingestuft werden. Da die potentielle Evapotranspiration im paraguayischen Chaco die $1140 \mathrm{~mm}$ übersteigt, wird das Klima zusätzlich als megathermisch charakterisiert (Grassi et al. 2005).

Globale Klimaschwankungen wie das Phänomen El Niño-Südliche Oszillation (ENSO) hat in Südamerika wiederholt zu extremen Niederschlägen und Überschwemmungen geführt (Grimm \& Tedeschi 2009). Erste Überlieferungen einer ungewöhnlichen Serie mehrerer Überschwemmungen in Südamerika, die womöglich auf das ENSO-Phänomen zurückzuführen ist, gehen laut Contreras et al. (2010) auf die Jahre 1525/26 zurück. In Paraguay sind EL Niño-Überschwemmungsphänomene meist mit extremen Hochwasserständen im Paraguay-Paraná-Becken verbunden und daher auch überliefert worden.

Die Phasen dieser Klimaschwankungen, wie „El Niño“ und das Gegenteil „La Niña“ führen nach Barros et al. (2008) zu unterschiedlichen Niederschlagstendenzen im südlichen Südamerika. Im Chaco Boreal kommt es daher einerseits zu periodischen Zunahmen an Niederschlägen und andererseits zu extrem niederschlagsarmen Jahren. Besonders intensive El Niño-Phänomene wurden im Zentralen Chaco in den Jahren 1982/83, 1992, 1998 und 2012 beobachtet. In den Jahren 1981, 2002 und 2010 wurden im Gegensatz dazu extrem niedrige Niederschlagsmengen verzeichnet.

Basierend auf Mittelwerten von vier globalen Klimamodellen (aus Solomon et al. 2007) prognostizieren Bidegain et al. (2012) für den paraguayischen Chaco einen Temperaturanstieg von $1,0^{\circ} \mathrm{C}$ bis $1,3^{\circ} \mathrm{C}$ für $2020,2,0^{\circ} \mathrm{C}$ bis $2,5^{\circ} \mathrm{C}$ für 2050 und $4,0^{\circ} \mathrm{C}$ bis $4,8^{\circ} \mathrm{C}$ für 2080 . Niederschlagsprognosen zeigen für 2020 eine Zunahme von 3\% im Süden des paraguayischen 
Chaco und eine Abnahme von 6\% im nördlichen Chaco. Für 2050 werden Änderungen im Niederschlagsregime von $+5 \%$ bis $-9 \%$ und für 2080 von $-5 \%$ bis $-20 \%$ prognostiziert. Die höchsten Temperaturanstiege und Niederschlagsreduzierugen würden jeweils im nördlichen Chaco und dem oberen Flussgebiet des Río Paraguay registriert werden.

\subsection{Engeres Untersuchungsgebiet}

Das Untersuchungsgebiet (Abb. 2-6) liegt in der Zone der Binnenlandsalzstandorte im Chaco

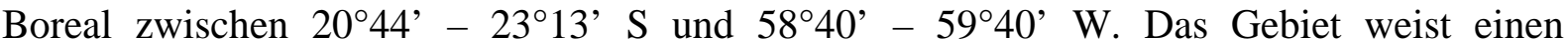
Feuchtegradienten in Ost-West-Richtung und einen Temperaturgradienten in Nord-SüdRichtung auf. Im Untersuchungsgebiet befinden sich mehrere periodisch wasserführende Flüsse mit einer großen Anzahl von Salzlagunen, Mäandern und salzreichen Einschwemmsenken. Aufgrund der vielen repräsentativen Salzstandorte und seiner Lage in der teilweise stark versalzungsgefährdeten Übergangszone (Abb. 2-2) von den regelmäßig überschwemmten Flächen des Bajo Chaco zum trockenen Zentralen Chaco wurde dieses Gebiet für die Untersuchungen ausgewählt. Zwischen den Breitengraden $22^{\circ}$ und $23^{\circ} \mathrm{S}$ werden die natürlichen Versalzungsprozesse sehr stark von anthropogener Nutzung beeinflusst, während die Versalzungsphänomene im nördlichen Gebiet größtenteils rein natürlichen Ursprungs sind.

Die Tabelle 2-1 zeigt die chemischen Eigenschaften des Oberflächenwassers einiger periodisch wasserführenden Flusssysteme im Untersuchungsgebiet. Die Wasserproben wurden während der Geländearbieten 2011 und 2012 aus den jeweiligen Gewässern entnommen und im Labor der Facultad de Ciencias Químicas der Nationalen Universität von Asunción analysiert. Laut Wiebe (2003) spiegelt die Salzzusammensetzung der Lagunenstandorte die Salze des Grundwassers wieder, wo der Gesamtanteil der Natriumsalze über 80\% steigen kann. Die Hauptsalze im Boden der höher gelegenen Flächen sind Chloride und Sulfate des Natriums, Calciums und Magnesiums, wobei auch die Natriumsalze die Hauptfraktion bilden.

Tab. 2-1: Chemische Eigenschaften des Oberflächenwassers einiger periodisch wasserführenden Flusssysteme im Untersuchungsgebiet.

\begin{tabular}{lllrrrrrrr} 
& Datum & $\mathrm{pH}$ & $\begin{array}{c}\text { Elek. Leit. } \\
(\mathrm{mS} / \mathrm{cm})\end{array}$ & $\begin{array}{r}\text { Härte } \\
(\mathrm{mg} \text { CaCO3/l) }\end{array}$ & $\begin{array}{c}\text { Calcium } \\
(\mathrm{mg} / \mathrm{l})\end{array}$ & $\begin{array}{r}\text { Magnesium } \\
(\mathrm{mg} / \mathrm{l})\end{array}$ & $\begin{array}{c}\text { Chlorid } \\
(\mathrm{mg} / \mathrm{l})\end{array}$ & $\begin{array}{c}\text { Sulfat } \\
(\mathrm{mg} / \mathrm{l})\end{array}$ & $\begin{array}{c}\text { Natrium } \\
(\mathrm{mg} / \mathrm{l})\end{array}$ \\
\hline Riacho Florida & 25.11 .2011 & 6.3 & 27.0 & 9485.0 & 3095.0 & 425.0 & 9139.0 & 8167.0 & \\
Riacho Curupayty & 17.01 .2012 & 6.5 & 6.5 & 654.5 & 148.7 & 68.8 & 1751.8 & 750.5 & 1024.6 \\
Riacho Mosquito & 17.01 .2012 & 6.7 & 7.5 & 670.6 & 155.1 & 68.8 & 1982.3 & 761.6 & 1319.4 \\
Campo Maria & 15.11 .2012 & 9.2 & 37.1 & 3604.0 & 407.0 & 629.0 & 9710.0 & 7471.8 & 5804.5 \\
Chaco Lodge & 25.11 .2012 & 9.0 & 53.7 & 5321.9 & 678.4 & 881.6 & 16021.5 & 12065.9 & 5914.3 \\
Riacho González & 22.11 .2012 & 8.9 & 101.6 & 12889.6 & 1051.5 & 2495.4 & 33985.0 & 36654.8 & 12528.2 \\
Rio Verde & 13.01 .2012 & 6.9 & 3.5 & 476.7 & 80.8 & 66.8 & 714.6 & 549.4 & 636.3 \\
\hline
\end{tabular}




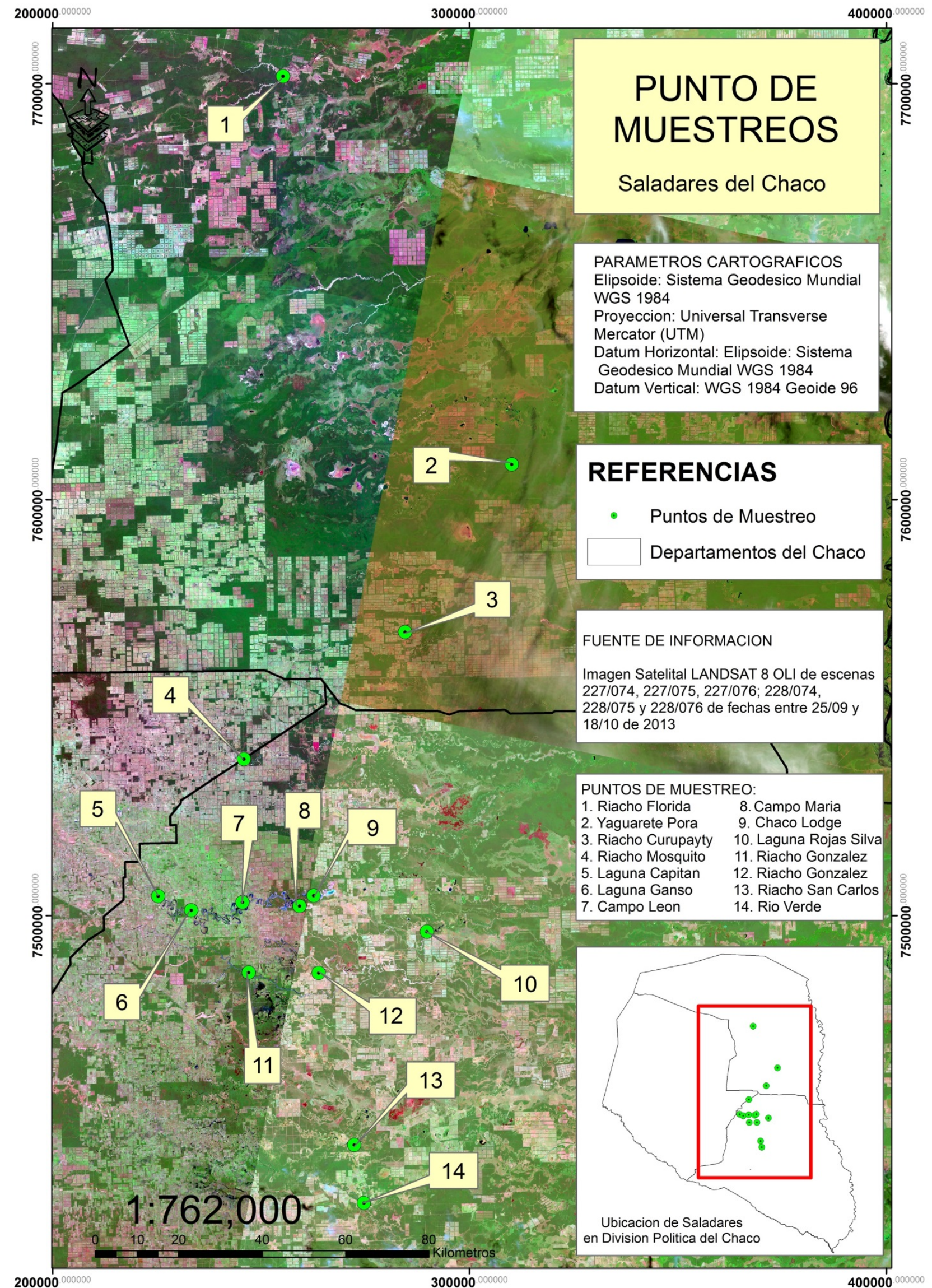

Abb. 2-6: Untersuchungsgebiet mit den einzelnen Untersuchungsorten (Satellitenkarte LANDSAT 8 OLI) 


\subsubsection{Untersuchungsorte im Departamento Alto Paraguay}

Reserva Natural Riacho Florida: Das private Naturschutzreservat, das der Fundación para el Desarrollo Sustentable del Chaco (DeSdelChaco) gehört, hat eine Größe von 1075 ha und befindet sich am südlichen Flussarm des periodisch wasserführenden Riacho Florida (2046’03’'S; 59²1'03’’W). Größtenteils aus salzbeeinflussten Standorten bestehend, repräsentiert das Naturreservat die nördliche Grenze der Binnenlandsalzstandorte im paraguayischen Chaco. Nach ersten Bestandsaufnahmen in den Jahren 2007/2008 wurde beim Nationalen Umweltsekretariat die Anerkennung des Status „Reserva Natural Privada“ beantragt.

> Reserva Natural Yaguarete Porã: Das seit 2008 staatlich anerkannte private Naturreservat der Firma Yaguarete Porã S.A. ist Teil des Biosphärenreservats des paraguayischen Chaco (21³7’00’S; 5850’03’’W). Mit einer Größe von 27.508 ha wird in diesem Gebiet eine naturbelassene und repräsentative Fläche der Binnenlandsalzstandorte unter Schutz gestellt. Aufgrund hoher Niederschläge während der Geländearbeiten konnten hier nur salzbeeinflusste Randgebiete untersucht werden.

$>$ Riacho Curupayty, Zone Centinela $\quad\left(21^{\circ} 58^{\prime} 39,74\right.$ '’'s; $\left.59^{\circ} 05^{\prime} 12,78^{\prime \prime} \mathrm{W}\right)$ : In der Umgebung des Polizeistützpunktes Centinela wurden mehrere Salzstellen des periodisch wasserführenden Riacho Curupayty untersucht (Abb 2-7).

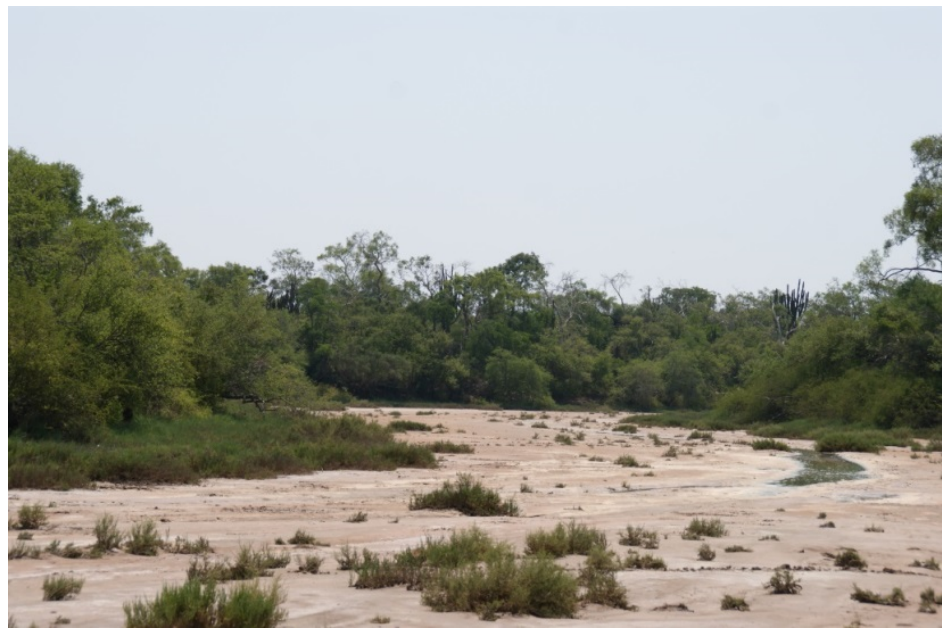

Abb. 2-7: Salzhaltiges Flussbett des Riacho Curupayty

\subsubsection{Untersuchungsorte im Departamento Presidente Hayes}

$>$ Riacho Mosquito, Zone Estancia Pozo Azul (22¹452,87’'S; 59²7’56,47’'W): am periodisch wasserführenden Riacho Mosquito wurden die Probeflächen aufgrund mangelnder Zugangsmöglichkeiten direkt an einer verkehrsreichen Straße anlegt.

$>$ Laguna Capitán (22³2'33'’S; 5940’15'’W) ist ein kleinflächiges Naturreservat der Genossenschaft Chortizer Komitee. Geprägt von starken anthropogenen Einflüssen, wie Abdämmung und großflächige Weiden, weist dieser Ort extrem versalzene Standorte auf. Laguna Capitán gehört zusammen mit Laguna Ganso, Campo León, Campo María, Chaco Lodge und Laguna Rojas Silva zum Feuchtgebiet des Riacho Yakaré Sur. 
Laguna Ganso (22³4'27''S; 59 $35^{\prime} 39^{\prime \prime}$ 'W) ist ein aus Privatinitiative geschütztes Gebiet, das den Familien Derksen und Friesen gehört. Umringt von großen Weideflächen, bietet dieses ca. 1000 ha große Reservat eine Schutzzone für die typische Flora und Fauna der salzbeeinflussten Habitate am Riacho Yakaré Sur (Abb. 2-8).

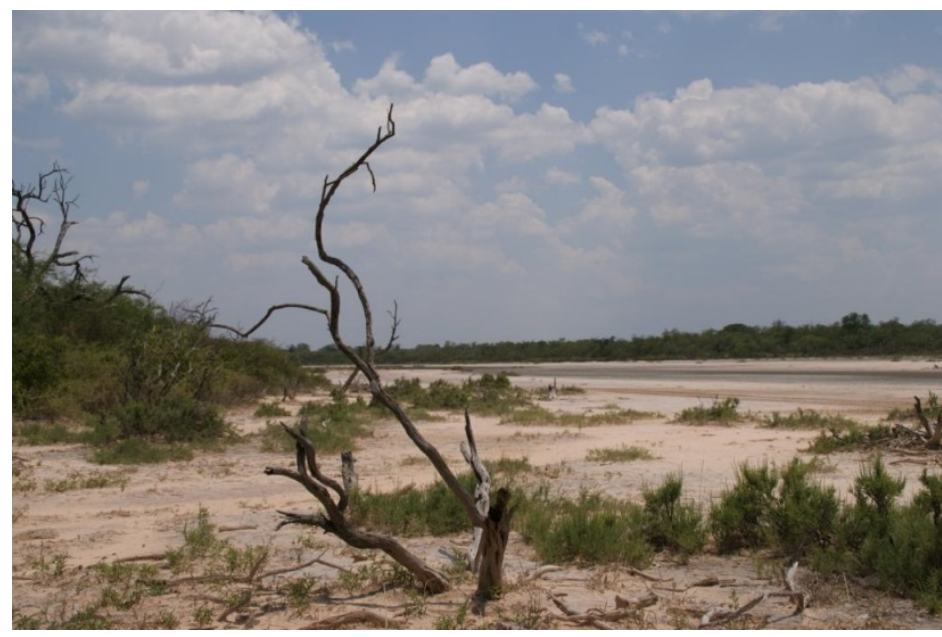

Abb. 2-8: Salzstandorte in der Umgebung der Laguna Ganso

$>$ Campo León und Umgebung (22³3’31“'S; 59²8’28“'W): hier wurden verschiedene Salzstellen untersucht, die unter starken anthropogenen Einflüssen, wie Wegebau und Weideflächgen, stehen.

Reserva Natural Campo Maria $\left(59^{\circ} 20 ' 22^{\prime \prime} \mathrm{W}, 22^{\circ} 34^{\prime} 09^{\prime \prime}\right.$ S) ist ein privates Naturschutzreservat der Genossenschaft Chortizer Komitee mit einer Größe von 3829 ha. In diesem Naturschutzgebiet befinden sich eine große Anzahl von Mäandern und kleinen Salzlagunen, die Teil des Riacho Yakaré Sur sind (Abb. 2-9)

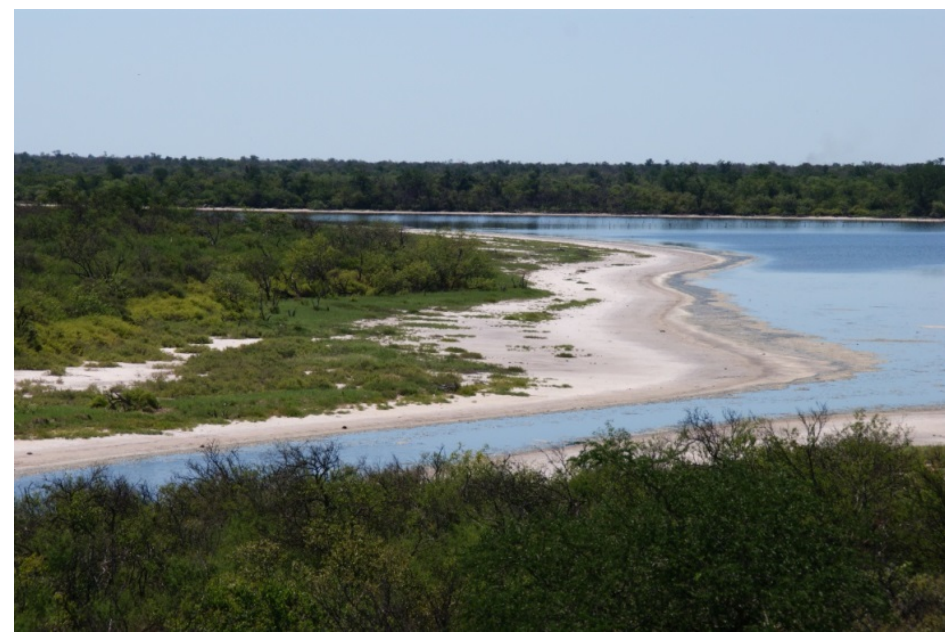

Abb. 2-9: Riacho Yakaré Sur in der Reserva Natural Campo Maria

Reserva Natural Chaco Lodge $\left(59^{\circ} 18^{\prime} 00^{\prime \prime} \mathrm{W}, 22^{\circ} 33^{\prime} 03^{\prime \prime} \mathrm{S}\right)$ ist ein 2500 ha großes Feuchtgebiet, das am 20. Oktober 2003 als Ramsar-Gebiet Nr. 1330 erklärt wurde. Die Salzlagune „Laguna Flamenco“ im Schutzgebiet zählt mit einer Größe von 500 ha zu den größten dieser Art im Chaco Boreal (Abb. 2-10).

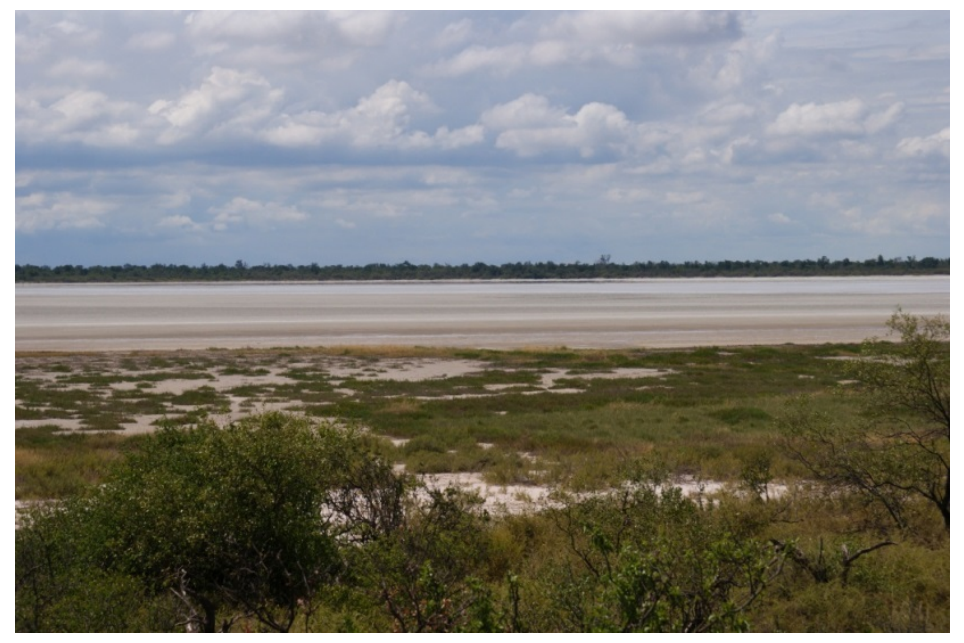

Abb. 2-10: Die Laguna Flamenco im Ramsargebiet Chaco Lodge war im Februar 2010 größtenteils ausgetrocknet. 
Laguna Teniente Rojas Silva (22³7’42'’S; 5902’44’'W) ist seit 2004 als Ramsar-Gebiet international anerkannt. Das 8.470 ha große Reservat gehört der Indianersiedlung von Pozo Amarillo.

> Der Riacho González ist ein extrem salzhaltiges Flussbett, das sich südlich des Riacho Yakaré Sur befindet. Probeflächen wurden in der Zone des Dorfes Loma Verde

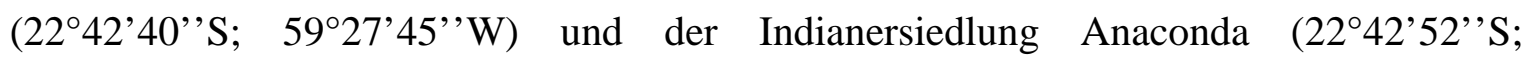
59॰17’58’’W) angelegt und untersucht.

$>$ Reserva Natural Fortín Salazar: Das seit 2008 staatlich anerkannte private Naturreservat der Firmen ARPA S.A.C.I.A und EATON \& CIA S.A, befindet sich an der Ruta Transchaco in der Zone des Kilometersteins 320 (2305'20'’S; 59¹3'22'’W). In dem 12.450 ha großen Schutzgebiet werden typische hydromorphe alkali- und salzbeeinflusste Standorte am Riacho San Carlos geschützt.

> Rio Verde, Ruta Transchaco am Kilometerstein 300 (23¹2'55'’'S; 59¹2'07' 'W): der Rio Verde sieht mit seinen steilen Uferhängen ganz anders aus als die meisten periodisch wasserführenden Flusssysteme im trockenen Chaco. Doch auch in der untersuchten Zone führt er nur zu Regenzeiten Wasser. Während im Flussbett typische SalzmarschGesellschaften auftreten, ist die Ufervegetation hauptsächlich von Xerophyten geprägt. 


\section{Phytogeographische Charakterisierung und Lebensformen- spektrum der Flora salzbeeinflusster Habitate im Chaco Boreal, Paraguay}

\subsection{Einleitung}

Die klassische biogeographische Einteilung Lateinamerikas von Cabrera \& Willink (1973) ist bis heute wohl die am häufigsten verwendete Klassifikation der Ökoregionen. Weitere klassische biogeographische Klassifikationsansätze des südamerikanischen Subkontinents wurden von Hueck (1966), Udvardy (1975), Takhtajan (1986) und Dinerstein et al. (1995) veröffentlicht. Jedoch wurden von diesen Autoren die disjunkt verbreiteten Saisontrockenwälder in der Mitte Südamerikas nicht als eigenständige Florenregion behandelt. Erst in neueren Ansätzen (Prado \& Gibbs 1993, Prado 2000) werden die Neotropischen Saisontrockenwälder, die als disjunkte Reliktvorkommen des Pleistozäns verstanden werden können, als separate Florenregion für die Neotropis genannt.

In Anlehnung an Cabrera (1970), Cabrera \& Willink (1973), Prado (2000) und Oakley \& Prado (2011) wird die Flora Paraguays von drei Florenregionen geprägt: Chaco-Florenregion („Dominio Chaqueño“), Amazonas-Florenregion („Dominio Amazónico“) und die Florenregion der Neotropischen Saisontrockenwälder („Dominio de los Bosques Secos Estacionales Neotropicales").

Laut Spichiger et al. (2005) ist Paraguay als ein Übergangsgebiet anzusehen, in dem mehrere Floren und Vegetationstypen konkurrieren. Die fehlende Isolation und die damit verbundene geringe Artbildung sind auf die biogeographische Lage des Paraguay-Paraná Beckens und das schwach ausgeprägte topographische Relief zurückzuführen. Die großen Flüsse wie der Río Paraná, der Río Paraguay oder der Río Pilcomayo und einige ihrer Nebenflüsse dienten der heutigen Flora und Fauna als Migrationswege (Bernardi 1984).

Klimatisch und floristisch wird der paraguayische Chaco meist in einen trockenen Teil, den „Chaco seco“, und einen feuchten Teil, den „Chaco húmedo“, geteilt (Ramella \& Spichiger 1989, Spichiger et al. 1991, 1995, 2005). Da die Flora und Vegetation sehr eng mit der Alkalinität, Salinität und Textur des Bodens verbunden sind, kann die Chacovegetation als edaphoklimatisch definiert werden (Spichiger et al. 1995).

Die floristische Komposition salzhaltiger Standorte ist selbst in unterschiedlichen Regionen recht ähnlich, jedoch werden die Übergangszonen, in denen die Salinität abnimmt, hauptsächlich von der jeweiligen phytogeographischen Region geprägt (Nebbia \& Zalba 2007).

Im folgenden Kapitel wird die Flora der salzbeeinflussten Standorte im paraguayischen Chaco anhand der taxonomischen Diversität, des Lebensformenspektrums und der phytogeographischen Einordnung untersucht. Zum direkten Vergleich wird dafür die Checkliste der Gefäßpflanzen des paraguayischen Chaco (Vogt 2011, 2012, 2013) verwendet. 


\subsection{Methoden}

\subsubsection{Checkliste der Gefäßpflanzen im paraguayischen Chaco}

Die Liste der im paraguayischen Chaco vorkommenden Pflanzenarten wurde mit Hilfe des Gefäßpflanzen-Katalogs vom Cono Sur (Zuloaga et al. 2008), der Flora del Paraguay, des Catalogus Hasslerianus (Ramella 2008, 2009, 2010, 2011) und den OnlineHerbardatenbanken (CHG 2013; IRIS 2013; KEW 2013; NHM 2013; NYBG 2013 und Tropicos 2013) erstellt. Die Checkliste der Gefäßpflanzen des paraguayischen Chacos wurde in drei Teilen veröffentlicht: Pteridophyta und Monocotyledoneae (Vogt 2011), Dicotyledoneae: Acanthaceae - Fabaceae (Vogt 2012) und Dicotyledoneae: Gesneriaceae Zygophyllaceae (Vogt 2013).

Die Nomenklatur der Pflanzenfamilien richtet sich nach Stevens 2001 [continuously updated]) und Angiosperm Phylogeny Group (2009) und der Arten nach Zuloaga et al. (2008).

Für jede Art wurde der floristische Status nach folgenden Kriterien ermittelt: heimische Arten (NAT), endemische Arten des Südkegels (Cono Sur) von Südamerika (ENDCS), endemische Arten vom Gran Chaco (ENDGC), endemische Arten vom paraguayischen Chaco (END), und eingebürgerte Arten (Natur).

Anhand von Herbaraufsammlungen, floristischen Beschreibungen und eigenen Beobachtungen wurden den Arten je nach Verbreitung die jeweilige Einteilung des Niederschlagsgradienten im paraguayischen Chaco (Tab. 3-1) zugeordnet.

Tab. 3-1: Einteilung des Niederschlagsgradienten im paraguayischen Chaco.

\begin{tabular}{llll} 
Abkürzung & Niederschlagszone & Mittlere Jahresniederschläge & Abgrenzung \\
\hline $\mathrm{H}$ & Feuchter Chaco & $1000-1400 \mathrm{~mm}$ & Ostgrenze ist der Rio Paraguay \\
$\mathrm{S}$ & Trockener Chaco & $700-1000 \mathrm{~mm}$ & Ostgrenze: $59^{\circ} \mathrm{W}$ \\
$\mathrm{A}$ & Semiarider Chaco & $400-700 \mathrm{~mm}$ & Ostgrenze: $61^{\circ} \mathrm{W}$ \\
\hline
\end{tabular}

\subsubsection{Artenliste der salzbeeinflussten Habitate im paraguayischen Chaco}

Grundlage für die Artenliste des Untersuchungsgebietes (Anhang 3-2) sind 236 randomisierte Vegetationsaufnahmen in der Gehölzvegetation salzbeeinflusster Standorte und 162 Vegetationsaufnahmen in den Offenland-Salzstellen. Von den Arten wurden Herbarbelege angefertigt, die im Herbarium FCQ (Departamento de Botánica de la Facultad de Ciencias Químicas, Universidad Nacional de Asunción) nachbestimmt und hinterlegt wurden. Dubletten werden zudem in den Herbarien CTES, FACEN, G und GOET hinterlegt.

\subsubsection{Lebensformenspektrum}

Für die Arten im paraguayischen Chaco wurde erstmalig eine Charakterisierung der Lebensformen nach Ellenberg \& Mueller-Dombois (1967) vorgenommen. Wie von diesen 
Autoren vorgeschlagen, wurde aufgrund des tropisch-subtropischen Klimas für die Chamaephyten eine maximale Höhe von $1 \mathrm{~m}$ verwendet.

\subsubsection{Phytogeographische Einordnung}

In Anlehnung an Floren und Online-Datenbanken (in 3.2.1. zitiert) wurde für jede Art die heutige geographische Verbreitung ermittelt und so die phytogeographische Einordnung (Tab. 3-2) definiert.

Tab. 3-2: Phytogeographische Elemente in Anlehnung an Cabrera \& Willink (1973), López \& Beck (2002) und Oakley \& Prado (2011).

Phytogeographische Verbreitung

Kosmopolitisch (KOS)

Paläotropisch (PAL)

Pantropisch (PAN)

Amerikanisch (AMER)

Amphitropisch (AMPHI)

Neotropisch (NEO)

Disjunkt Neotropisch (DISNEO)

Florenregionen südlich des Amazonas (SURAMA)

Meridionales Andenvorland (VORAND)

Florenregion Neotropische Saisontrockenwälder (DOMNSTW)

Florenregion Chaco (DOMCHA)

Gran Chaco (CHA)

\section{Definition}

Weite geographische Verbreitung in mehreren Kontinenten

Ursprung in tropischen und subtropischen Gebiete der Alten Welt

Tropische und subtropische Gebiete in mindestens zwei Kontinenten

Weite Verbreitung in Amerika (auch gemäßigte Gebiete)

Disjunkte Verbreitung in subtropischen und gemäßigten Gebieten Nord- und Südamerikas, jedoch fehlend in den Tropen dazwischen. In den meisten Fällen handelt es sich um Arten der Trockengebiete

Mittel- und Südamerika einschließlich der südlichen außertropischen Gebiete sowie Westindien

Disjunkte Verbreitung in der Neotropis, mindestens in zwei Florenregionen

In mindestens zwei Florenregionen südlich des Amazonas in: Paraguay, Süd- und Südostbrasilien, Uruguay, Nord- und Zentralargentinien, Nordchile, Bolivien und Südperú

Mindestens in zwei Florenregionen im östlichen Andenvorland in der Mitte Südamerikas. Folgende Florenprovinzen gehören dazu: Gran Chaco, Monte, Pedemonte, Prepuna, Puna

Der sogenannte Pleistozän-Bogen reicht von den Caatingas im Nordosten Brasilien über den Südosten Brasiliens bis zum Zusammenfluss der Flüsse Paraguay und Paraná, dem Südwesten Boliviens und Nordwesten Argentiniens und erstreckt sich sporadisch in die trockenen Andentälern Perus und an die Westküste Ecuadors

Südlichste Florenregion der Neotropis bestehend aus den Florenprovinzen Chaco, Espinal, Monte, Pampa und Prepuna Umfasst die gesamte Chaco-Florenprovinz 


\subsection{Ergebnisse}

\subsubsection{Taxonomische Diversität}

Die Checkliste vom paraguayischen Chaco enthält 1680 Arten und 68 Unterarten, die in 652 Gattungen und 126 Familien verteilt sind (Vogt 2011, 2012, 2013). An salzbeeinflussten Standorten wurden davon 318 Arten (18,9 \%), 214 Gattungen (38,1 \%) und 63 Familien (50 \%) gefunden. Die gattungs- und artenreichsten Familien sind Fabaceae, Poaceae, Asteraceae und Malvaceae (Tab. 3-3, Anhang 3-1 \& 3-2). Die Rangordnung der Familien beider Listen ist recht ähnlich. Die Familien Cyperaceae und Orchidaceae haben einen hohen Stellenwert im feuchten Chaco und wurden daher an salzbeeinflussten Standorten kaum gefunden. Die hohe Rangordnung der Kakteen und Bromelien an Salzstandorten geht auf weitverbreitete Arten im Chaco zurück.

165 Arten (10,1 \%) der heimischen Arten im paraguayischen Chaco sind Regionalendemiten; davon wurden 28 (17\%) an den Salzstandorten gefunden. Von den 45 Endemiten des paraguayischen Chaco kommen 5 (11,1 \%) in den salzbeeinflussten Habitaten vor (Tab. 3-4).

Tab. 3-3: Die wichtigsten Familien im paraguayischen Chaco (a) und den salzbeeinflussten Standorten (Saladares) (b).
a) Paraguayischer Chaco
Familien: 126
Gattungen: 652
Arten: 1680

b) Saladares

Familien: 63

Gattungen: 214

Arten: 318

\begin{tabular}{lrrrrrrrrr} 
Familie & $\mathbf{N}^{\circ} \mathbf{S p}$ & $\mathbf{\%}$ & $\mathbf{N}^{\circ} \mathbf{G e n}$. & $\mathbf{\%}$ & Familie & $\mathbf{N}^{\circ} \mathbf{S p}$. & $\mathbf{\%}$ & $\mathbf{N}^{\circ} \mathbf{G e n}$. & $\mathbf{\%}$ \\
\hline Fabaceae & 177 & 10.5 & 60 & 9.2 & Fabaceae & 43 & 13.5 & 27 & 12.6 \\
Poaceae & 171 & 10.2 & 55 & 8.4 & Poaceae & 25 & 7.9 & 19 & 8.9 \\
Asteraceae & 164 & 9.8 & 64 & 9.8 & Asteraceae & 21 & 6.6 & 17 & 7.9 \\
Malvaceae & 107 & 6.4 & 28 & 4.3 & Malvaceae & 21 & 6.6 & 12 & 5.6 \\
Euphorbiaceae & 75 & 4.5 & 18 & 2.8 & Cactaceae & 16 & 5.0 & 11 & 5.1 \\
Solanaceae & 56 & 3.3 & 14 & 2.1 & Euphorbiaceae & 16 & 5.0 & 9 & 4.2 \\
Cyperaceae & 53 & 3.2 & 14 & 2.1 & Bromeliaceae & 16 & 5.0 & 6 & 2.8 \\
Apocynaceae & 51 & 3.0 & 23 & 3.5 & Solanaceae & 13 & 4.1 & 5 & 2.3 \\
Amaranthaceae & 47 & 2.8 & 15 & 2.3 & Amaranthaceae & 12 & 3.8 & 8 & 3.7 \\
Convolvulaceae & 41 & 2.4 & 9 & 1.4 & Apocynaceae & 10 & 3.1 & 7 & 3.3 \\
Verbenaceae & 38 & 2.3 & 10 & 1.5 & Acanthaceae & 10 & 3.1 & 5 & 2.3 \\
Cactaceae & 37 & 2.2 & 14 & 2.1 & Convolvulaceae & 8 & 2.5 & 5 & 2.3 \\
Acanthaceae & 32 & 1.9 & 9 & 1.4 & Bignoniaceae & 7 & 2.2 & 4 & 1.9 \\
Rubiaceae & 30 & 1.8 & 21 & 3.2 & Boraginaceae & 7 & 2.2 & 3 & 1.4 \\
Bromeliaceae & 30 & 1.8 & 9 & 1.4 & Cyperaceae & 6 & 1.9 & 4 & 1.9 \\
Orchidaceae & 27 & 1.6 & 21 & 3.2 & Verbenaceae & 5 & 1.6 & 4 & 1.9 \\
Bignoniaceae & 27 & 1.6 & 12 & 1.8 & Nyctaginaceae & 5 & 1.6 & 3 & 1.4 \\
Polygonaceae & 24 & 1.4 & 7 & 1.1 & Passifloraceae & 5 & 1.6 & 2 & 0.9 \\
Passifloraceae & 23 & 1.4 & 3 & 0.5 & Capparaceae & 4 & 1.3 & 4 & 1.9 \\
Sapindaceae & 22 & 1.3 & 12 & 1.8 & Sapindaceae & 4 & 1.3 & 3 & 1.4 \\
\hline
\end{tabular}


Bezüglich des westwärts abnehmenden mittleren Jahresniederschlags im paraguayischen Chaco kann eine Abnahme der Artenzahlen beobachtet werden (Abb. 3-1). 36,9 \% der Arten kommen nur im feuchten Chaco vor, $18,5 \%$ nur im trockenen Chaco, und $4,3 \%$ der Arten sind auf den semiariden Chaco beschränkt. 20,3\% wachsen sowohl im feuchten als auch im trockenen Chaco, 10,6 \% kommen sowohl im trockenen Chaco als im semiariden Chaco vor und nur 8,7 \% der Arten wurden in allen drei Niederschlagszonen gefunden.

Die Salzstandorte sind in einem Übergangsgebiet vom feuchten zum trockenen Chaco verbreitet. 30,2\% der vorkommenden Arten wachsen sowohl im feuchten als auch trockenen Chaco, 24, 8 \% kommen in den drei Zonen des Niederschlagsgradienten vor und 21,4\% sind auf den trockenen und semiariden Teil des Chacos reduziert (Abb. 3-1).

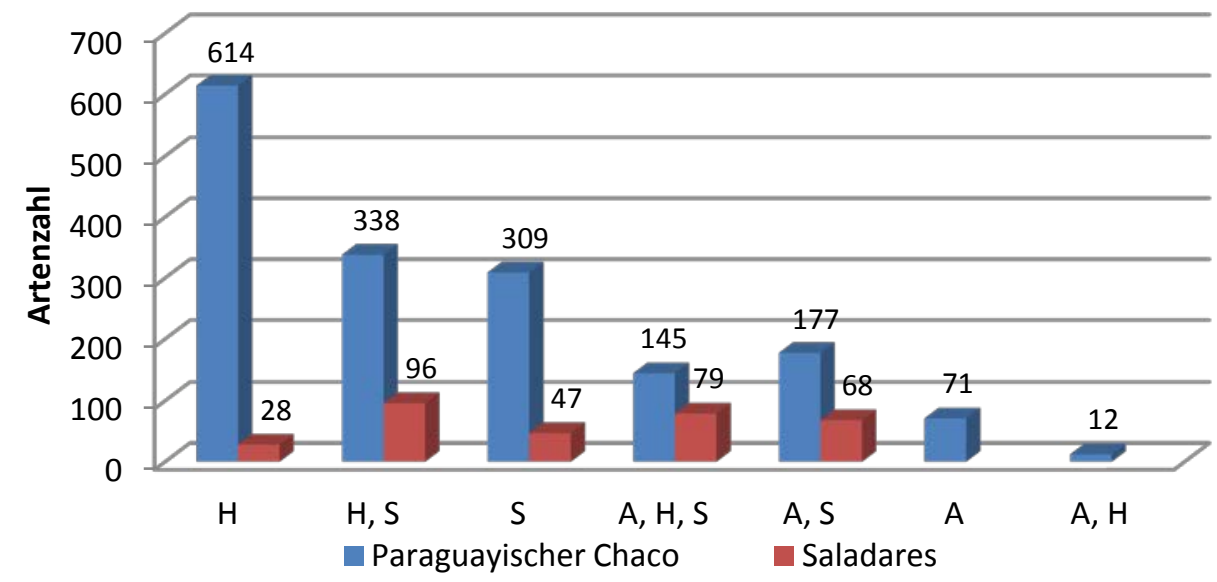

Abb. 3-1: Verteilung der Arten nach mittleren Jahresniederschlag im paraguayischen Chaco und den Saladares: H) feuchter Chaco (1000-1400 mm), S) trockener Chaco (700-1000 mm) und A) semiarider Chaco (400-700 mm).

\subsubsection{Lebensformenspektrum}

Im paraguayischen Chaco dominieren die Phanerophyten (30\%), gefolgt von den Chamaephyten (20 \%), Hemikryptophyten (16 \%) und Lianen (10 \%). Die gleiche Rangordnung konnte auch im Untersuchungsgebiet der salzbeeinflussten Habitate mit jeweils $33 \%, 25 \%, 16 \%$ und $9 \%$ beobachtet werden (Abb. 3-2). In den Salzmarschen dominieren Hemikryptophyten (32 \%) gefolgt von Chamaephyten (20\%) und Therophyten (18\%). 


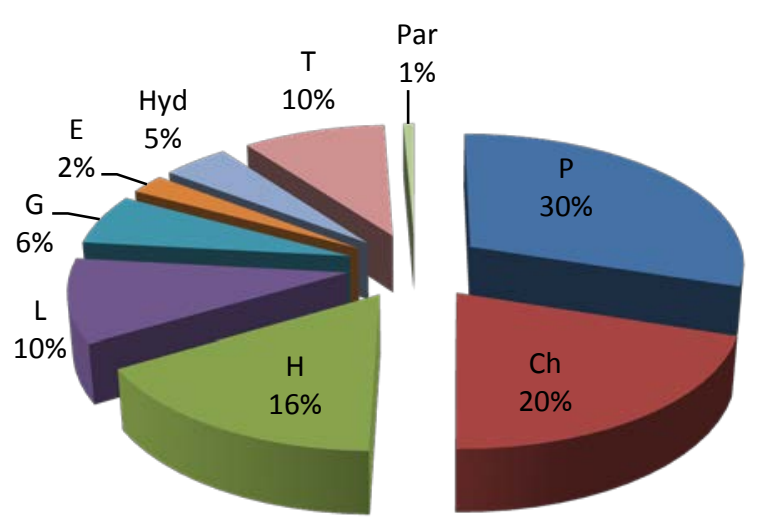

a)

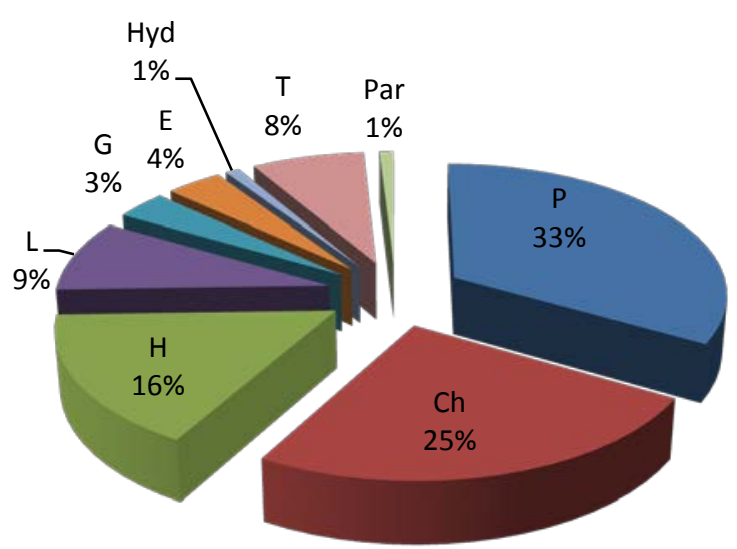

b)

Abb. 3-2: Lebensformenspektrum nach Raunkiaer im paraguayischen Chaco (a) und im Untersuchungsgebiet (b): Phanerophyten (P), Chamaephyten (Ch), Hemikryptophyten (H), Lianen (L), Geophyten (G), Epiphyten (E), Hydrophyten (Hyd), Therophyten (T) und Parasiten (Par).

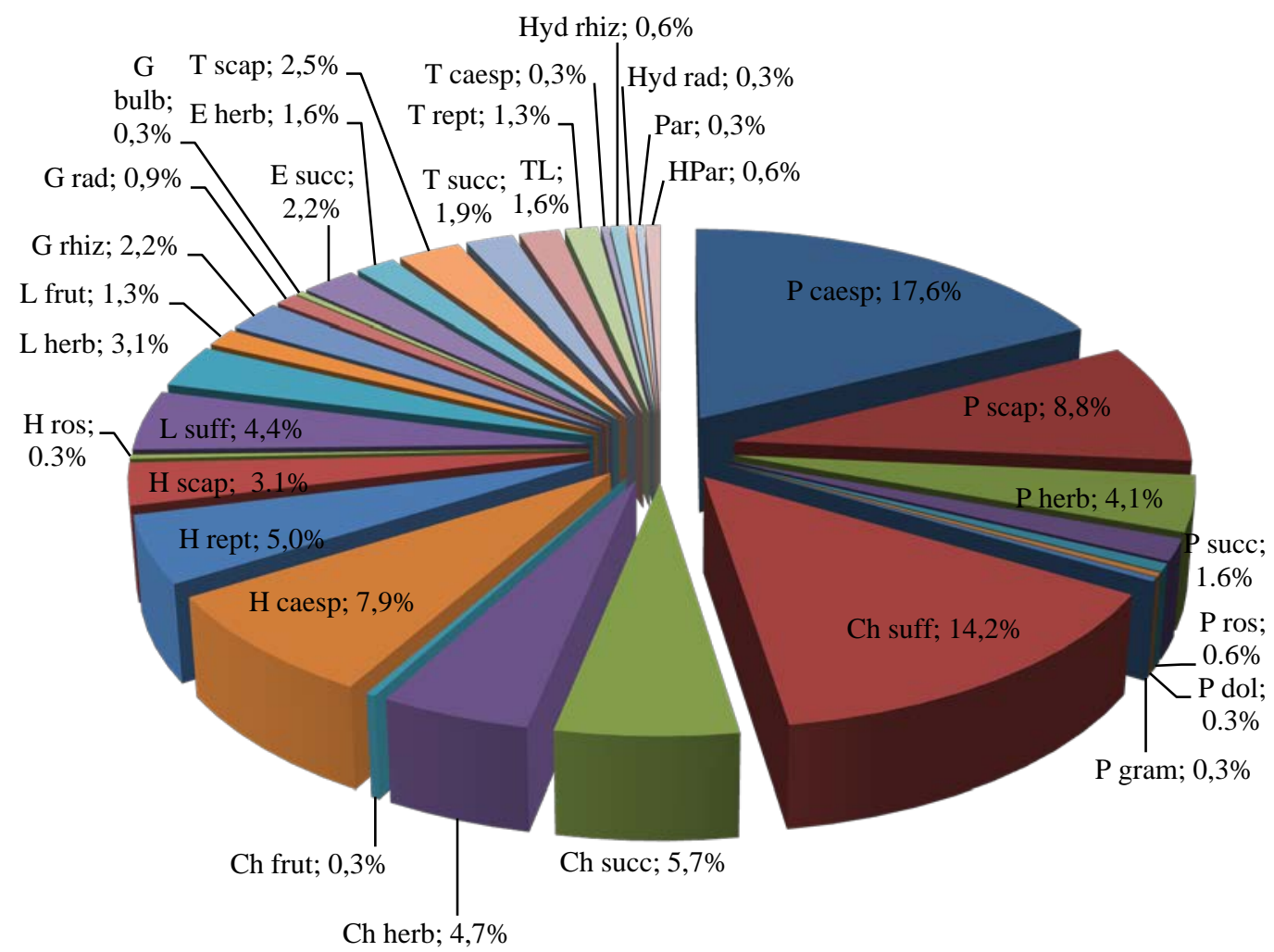

Abb. 3-3: Lebensformenspektrum nach Ellenberg \& Mueller-Dombois (1967) der Arten im Untersuchungsgebiet: Phanerophyten (P): Bäume (scap), Sträucher (caesp), Hochstauden (herb), hohe Sukkulenten (succ), rosettenartig (ros), Flaschenbäume (dol), graminoide (gram); Chamaephyten (Ch): halb-holzige Zwergsträucher (suff), niedrige Sukkulenten (succ), krautig (herb), holzige Zwergsträucher (frut); Hemikryptophyten (H): horstig (caesp), niederliegend (rept), aufrecht (scap), rosettenförmig (ros); Lianen (L): halb-verholzt (suff), krautig (herb), holzig (frut); Geophyten (G): Rhizomgeophyten (rhiz), Wurzelgeophyten (rad), Knollengeophyten (bulb); Epiphyten (E): sukkulent (succ), krautig (herb); Therophyten (T): aufrecht (scap), sukkulent (succ), rankend (TL), niederliegend (rept) und verzweigt (caesp); Hydrophyten (Hyd): Rhizomhydrophyten (rhiz), Wurzelhydrophyten (rad); Parasiten: Holoparasiten (Par) und Hemiparasiten (HPar). 
Im Untersuchungsgebiet dominieren die Sträucher (P caesp) mit 17,6 \% und die halbholzigen Zwergsträucher (Ch suff) mit 14,2 \% der Arten. Wichtige Anteile kommen außerdem den Bäumen (P scap) mit 8,8 \%, den horstigen Hemikryptophyten (H caesp) mit 7,9 $\%$ und den niedrigwüchsigen Sukkulenten (Ch succ) mit 5,7 \% zu (Abb. 3-3).

\subsubsection{Phytogeographische Charakterisierung}

In den salzbeeinflussten Habitaten im paraguayischen Chaco dominieren pantropische Gattungen (30,8 \%), gefolgt vom neotropischen (25,2 \%) und kosmopolitischen (19,6\%) Element. Auf Artenebene kommt dem Chaco-Element der größte Anteil zu (30,6 \%), gefolgt vom neotropischen Element (14,2 \%) und den Arten mit Verbreitung im Andenvorland (11,3 \%) (Tab. 3-5).

In den Salzmarschen ist das phytogeographische Spektrum größtenteils von kosmopolitischen (40 \%) und pantropischen (37,5 \%) Gattungen geprägt. Auf Artenebene ist keine klare Dominanz bestimmter phytogeographischer Elemente $\mathrm{zu}$ beobachten, dennoch wird die Rangordnung vom Chaco-Element (15,9\%) sowie vom neotropischen (13,6 \%) und amphitropischen Element (13,6 \%) angeführt. Wenn die neotropischen Teilelemente summiert werden, ist mit 61,4 \% eine klare Dominanz der neotropischen Arten zu erkennen (Tab. 3-5). Das Lebensformenspektrum nach Raunkiaer für die jeweiligen phytogeographischen Elemente wird in Tabelle 3-6 dargestellt. Die in den salzbeeinflussten Habitaten dominierenden Chacoelemente werden mit 42,1 \% von den Phanerophyten und 33,7 \% von den Chamaephyten repräsentiert. Wichtige Bestandteile der neotropischen Elemente sind die Chamaephyten (34,1\%) und die Phanerophyten (22, 7 \%). Die Elemente des Andenvorlandes, die dritte Gruppe in der Rangordnung, besteht mit 51, 4 \% aus Phanerophyten. Anders sieht es bei den pantropischen, paläotropischen und amerikanischen Elementen aus, die hauptsächlich von den Hemikryptophyten repräsentiert werden. Kosmopolitische Arten im Untersuchungsgebiet sind größtenteils Therophyten.

Tab. 3-5: Phytogeographische Einordnung des Untersuchungsgebietes der salzbeeinflussten Standorte (Saladares) (a) und der Salzmarschen (b). Dargestellt werden die Gattungen und Arten mit der jeweiligen Anzahl und dem prozentualen Anteil. Arten, die nur bis zur Gattungsebene bestimmt werden konnten, wurden nicht berücksichtigt. Definitionen für die jeweiligen phytogeographischen Elemente befinden sich in Tab. 3-2.

a) Saladares

\begin{tabular}{lrrrrrrrr} 
& $\mathbf{N}^{\circ} \mathbf{G e n}$ & $\mathbf{\%}$ & $\mathbf{N}^{\circ} \mathbf{S p}$ & $\mathbf{\%}$ & $\mathbf{N}^{\circ} \mathbf{G e n}$ & $\mathbf{\%}$ & $\mathbf{N}^{\circ} \mathbf{S p}$ & $\mathbf{\%}$ \\
\hline KOS & 42 & 19.6 & 5 & 1.6 & 16 & 40 & 3 & 6.8 \\
PAN & 66 & 30.8 & 13 & 4.2 & 15 & 37.5 & 2 & 4.5 \\
AMER & 12 & 5.6 & 7 & 2.3 & 1 & 2.5 & 3 & 6.8 \\
AMPHI & 1 & 0.5 & 10 & 3.2 & 1 & 2.5 & 6 & 13.6 \\
NEO & 54 & 25.2 & 44 & 14.2 & 3 & 7.5 & 6 & 13.6 \\
DISNEO & 9 & 4.2 & 20 & 6.5 & 1 & 2.5 & 5 & 11.4 \\
SURAMA & 7 & 3.3 & 28 & 9 & & 0 & 3 & 6.8 \\
VORAND & 9 & 4.2 & 35 & 11.3 & 2 & 5 & 1 & 2.3 \\
DOMNSTW & 4 & 1.9 & 22 & 7.1 & & 0 & 1 & 2.3 \\
DOMCHA & 2 & 0.9 & 28 & 9 & & 0 & 5 & 11.4 \\
CHA & 6 & 2.8 & 95 & 30.6 & 1 & 0 & 7 & 15.9 \\
PAL & 2 & 0.9 & 3 & 1 & 40 & 100 & 44 & 100
\end{tabular}


Tab. 3-6: Lebensformenspektrum für die jeweiligen phytogeographischen Elemente (\%). Darstellt werden Lebensformen nach Raunkiaer: Phanerophyten (P), Chamaephyten (Ch), Hemikryptophyten (H), Lianen (L), Geophyten (G), Epiphyten (E), Hydrophyten (Hyd), Therophyten (T) und Parasiten (Par). Definitionen für die jeweiligen phytogeographischen Elemente befinden sich in Tab. 3-2.

\begin{tabular}{lcrrrrrrrrr} 
& P & Ch & \multicolumn{1}{c}{ H } & \multicolumn{1}{c}{ L } & \multicolumn{1}{c}{ G } & E & Hyd & T & Par & TOTAL \\
\hline KOS & & & & & 20.0 & & 20.0 & 60.0 & & 100.0 \\
PAN & 15.4 & 7.7 & 46.2 & & & 7.7 & & 23.1 & & 100.0 \\
AMER & 14.3 & & 42.9 & & 14.3 & & & 28.6 & & 100.0 \\
AMPHI & 10.0 & 30.0 & 30.0 & & 10.0 & & 10.0 & 10.0 & & 100.0 \\
NEO & 22.7 & 34.1 & 6.8 & 15.9 & 6.8 & 2.3 & 2.3 & 6.8 & 2.3 & 100.0 \\
DISNEO & 20.0 & 35 & 30 & 5.0 & & & & 10.0 & & 100.0 \\
SURAMA & 25.0 & 17.9 & 21.4 & 25.0 & 3.6 & 3.6 & & 3.6 & & 100.0 \\
VORAND & 51.4 & 14.3 & 11.4 & 14.3 & 2.9 & 2.9 & & 2.9 & & 100.0 \\
DOMNSTW & 66.7 & 14.3 & 4.8 & 9.5 & & 4.8 & & 13.8 & & 100.0 \\
DOMCHA & 31.0 & 24.1 & 13.8 & 3.4 & 3.4 & 3.4 & & 2.1 & 6.9 & 100.0 \\
CHA & 42.1 & 33.7 & 10.5 & 4.2 & 1.1 & 6.3 & & & & 100.0 \\
PAL & & & 100.0 & & & & & & & 100.0 \\
\hline
\end{tabular}

Tab. 3-7: Verteilung der phytogeographischen Elemente unter den am häufigsten vorkommenden Lebensformen (\%). Lebensformen nach Ellenberg \& Mueller-Dombois (1967): Sträucher (P caesp), Bäume (P scap), Hochstauden (P herb), halbholzige Zwergsträucher (Ch suff), niedrigwüchsige Sukkulenten (Ch succ), krautige Chamaephyten (Ch herb), horstige Hemikryptophyten ( $\mathrm{H}$ caesp), niederliegende Hemikryptophyten (H rept) und halbholzige Lianen (L suff). Die Definition der phytogeographischen Elemente befinden sich in Tab. 3-2.

\begin{tabular}{lrrrrrrrrr} 
& P caesp & P scap & P herb & Ch suff & Ch succ & Ch herb & H caesp & H rept & L suff \\
\hline PAN & & 3.6 & 7.7 & & & & 16.7 & 7.1 & \\
AMER & 1.8 & & & & & & 8.3 & 7.1 & \\
AMPHI & 1.8 & & & 2.2 & & 14.3 & 8.3 & 7.1 & \\
NEO & 10.7 & 10.7 & & 22.2 & 5.6 & 28.6 & 4.2 & 7.1 & 23.1 \\
DISNEO & 3.6 & 3.6 & 7.7 & 8.9 & 5.6 & 7.1 & 12.5 & 21.4 & \\
SURAMA & 8.9 & & 15.4 & 4.4 & 11.1 & 7.1 & 16.7 & & 23.1 \\
VORAND & 16.1 & 10.7 & 23.1 & 11.1 & & & 8.3 & & 15.4 \\
DOMNSTW & 8.9 & 25.0 & 15.4 & 4.4 & 5.6 & & 4.2 & & 15.4 \\
DOMCHA & 8.9 & 7.1 & 15.4 & 4.4 & 5.6 & 28.6 & 8.3 & 14.3 & \\
CHA & 39.3 & 39.3 & 15.4 & 40.0 & 66.7 & 14.3 & 8.3 & 21.4 & 23.1 \\
PAL & & & & 2.2 & & & 4.2 & 14.3 & \\
\hline TOTAL & 100.0 & 100.0 & 100.0 & 100.0 & 100.0 & 100.0 & 100.0 & 100.0 & 100.0
\end{tabular}

Sträucher und halbholzige Zwergsträucher, die dominierenden Lebensformen im Untersuchungsgebiet, werden mit jeweils 39,3 \% und 40 \% vom Chacoelement repräsentiert (Tab. 37). Die vorkommenden Baumarten gehören größtenteils zum Chacoelement (39,3 \%) und zum Element der neotropischen Saisontrockenwälder (25 \%). 66,7 \% der vorkommenden niedrigwüchsigen 
Sukkulenten sind nur im Gran Chaco verbreitet. Im Gegensatz dazu sind die Hemikryptophyten und die halbholzigen Lianen größtenteils überregionalen phytogeographischen Elementen zugeordnet.

\subsection{Diskussion}

\subsubsection{Biologische Diversität im überregionalen Vergleich}

Im paraguayischen Chaco wurden 9,5 \% aller Gefäßpflanzenarten im Conosur (Zuloaga et al. 2008), 24 \% der geschätzten Artenvielfalt in Paraguay (Mereles 2007) und ungefähr die Hälfte der nach The Nature Conservancy et al. (2005) für den Gran Chaco geschätzten Pflanzenarten gefunden. Sowohl die Größe als auch die Artenanzahl des paraguayischen Chacos lassen sich sehr gut mit dem im Norden angrenzenden Pantanal (Pott et al. 2011) vergleichen.

Binnenlandsalzstandorte sind meist artenarm, wie Studien aus Argentinen (Ragonese 1951, Cantero 1999) und Nordamerika (Ungar 1972) belegen. An den untersuchten salzbeeinflussten Standorten wurden 18,9 \% und in den Salzmarschen nur 2,6 \% der Arten vom paraguayischen Chaco gefunden.

Fabaceae, Poaceae und Asteraceae sind die artenreichsten Familien sowohl im paraguayischen Chaco als auch im Gebiet der salzbeeinflussten Habitate. Die gleichen Familien, jedoch in unterschiedlicher Rangordnung, weisen auch im Conosur (Zuloaga et al. 2008), in Chile (Moreira-Muñoz 2007), in Argentinien (Zuloaga et al. 1999), in den argentinischen Salinas Grandes (Ragonese 1951) und im argentinischen Chaco Serrano der Provinz Córdoba (Giorgis et al. 2011) die höchste taxonomische Diversität auf.

Weltweit gehören die meisten Halophyten und salztoleranten Pflanzen, nach Schätzungen von Yensen (2008), den Chenopodioideae (Subfamilie der Amaranthaceae), Poaceae, Asteraceae und Fabaceae an. In den untersuchten Salzmarschen im Chaco sind die Gräser vorherrschend. Die Chenopodioideae sind nur mit drei Arten repräsentiert, diese kommen jedoch in sehr hoher Frequenz in den Salzmarschen vor. Im überregionalen Vergleich sind in der Salzflora an den Küsten der Bahía Blanca (Argentinien) (Nebbia \& Zalba 2007), in der Saladoniederung in der Provinz Buenos Aires (Vervoorst 1967), in den Salzstandorten im Zentrum Argentiniens (Cantero 1999) und im Salar Ascotán in den nordchilenischen Anden (Teillier \& Becerra 2003) die Poaceae und Asteraceae vorherrschend, während in der Halophytenvegetation der argentinischen Provinz Mendoza die Chenopodioideae dominiert (Therburg 1997).

Die Binnenlandsalzstellen sind sich physiognomisch nach Ungar (1972) weltweit ausgesprochen ähnlich. Nach Untersuchungen in Nordamerika und Europa überwiegen in diesen Standorten hauptsächlich Hemikryptophyten und Therophyten (Ungar 1972). In Binnensalzstellen der argentinischen Pampa kommen nach Cantero (1999) überwiegend Rhizomgeophyten und Hemikryptophyten vor. Die azonale Salzflora im Salar Ascotán in den nordchilenischen Anden wird nach Teillier \& Becerra (2003) auch von Hemikryptophyten dominiert, während die angrenzenden Berghänge überwiegend von Nanophanerophyten besiedelt werden. 
Im Untersuchungsgebiet kommen im Gegensatz dazu viele Sträucher in den salzbeeinflussten Standorten vor. Das Lebensformenspektrum hängt jedoch vom Versalzungsgrad des Bodens ab. In den Salzmarschen sind Hemikryptophyten und Chamaephyten vorherrschend und die angrenzenden salzbeeinflussten Standorte werden hauptsächlich von Sträuchern besiedelt. Ragonese (1951) weist darauf hin, dass das Lebensformenspektrum der halophilen Gesellschaften der Salinas Grandes überwiegend von Phanerophyten und Hemikryptophyten geprägt ist, während in den Salzgesellschaften der Pampa die Therophyten und Hemikryptophyten vorherrschend sind. Ähnlicher Nord-Süd-Unterschied wurde von Therburg (1997) auch in der Monte-Florenprovinz (Provinz Mendoza) beschrieben. Während im Nordosten der Provinz hauptsächlich Sträucher und Halbsträucher aspektbildend sind, kommen im Süden überwiegend Hemikryptophyten und Geophyten vor. Therburg (1997) vermutet, dass mit zunehmender Aridität auch der Anteil der Phanerophyten zunimmt. Die im Untersuchungsgebiet vorkommenden Phanerophyten sind jedoch größtenteils ChacoElemente (Tabelle 3-7) und weisen somit eine klare Verbindung zur phytogeographischen Umgebung auf. Ausgehend vom überregionalen Vergleich ist daher anzunehmen, dass zunehmende Sonneneinstrahlung und Mitteltemperatur zu einem höheren Anteil der Phanerophyten in salzbeeinflussten Standorten führen.

\subsubsection{Phytogeographische Charakterisierung}

Die untersuchten salzbeeinflussten Standorte werden auf Gattungsebene von pantropischen und neotropischen Gattungen dominiert (Tab. 3-5). Prosopis, Acacia und Croton gehören zu den pantropisch verbreiteten Gattungen mit den meisten Vertretern im Untersuchungsgebiet, während Tillandsia die artenreichste neotropische Gattung im Untersuchungsgebiet ist.

Die neotropischen Trockengebiete weisen laut Sarmiento (1975) und López et al. (2006) eine klare floristische Diskontinuität in den zentral-peruanischen Anden auf und können in einen nördlichen tropischen und einen südlichen subtropisch-gemäßigten Teil getrennt werden. Obwohl der Chaco nach López et al. (2006) floristisch eine sehr große Ähnlichkeit mit der südlichen Trockenflora hat, scheint er eine gewisse Verbindung zwischen der südlichen und der nördlichen Flora zu bilden. Dieses wird im Untersuchungsgebiet durch den hohen Anteil, sowohl auf Gattungs- als auch auf Artenebene, des neotropischen Elementes bestätigt. Andererseits weist das Gebiet durch das Auftreten von Arten der Chaco-Florenregion (DOMCHA) und des Andenvorlandes (VORAND) Ähnlichkeit zu den südlichen subtropischgemäßigten Trockenregionen auf.

Auf Artenebene ist das Chacoelement mit 30,6 \% im Untersuchungsgebiet vorherrschend. Die hier verwendete Florenprovinz des Gran Chaco (CHA) entspricht der „Provincia Chaqueña“ nach Cabrera \& Willink (1973), der „Chacoan Province“ nach Takhtajan (1986) und den „Waldregionen des Chaco und seiner Randgebiete“ nach Hueck (1966). Die im trockenen Chaco verbreiteten xeromorphen Wälder und Gebüsche bestehen größtenteils aus Arten vom Gran Chaco (Prado 1993b). Es handelt sich nach Spichiger et al. (2004) um eine Klimaxvegetation mit einer einheitlichen Artenzusammensetzung als Folge der salzbeeinflussten Böden. Der feuchte Chaco ist nach Spichiger et al. (1995, 2005, 2006) ein Ökoton, in dem mehrere Elemente vermischt sind: Baumarten vom trockenen Chaco, Arten der Feuchtsavannen, mesophile weit verbreitete Arten und einige Elemente von den 
neotropischen Saisontrockenwäldern. In der Verteilung der Arten entlang des Niederschlagsgradienten wurde daher auch eine sehr hohe Artenanzahl im feuchten Chaco gefunden (Abb. 3-1).

Interessant sind die Taxa der neotropischen Saisontrockenwälder im Untersuchungsgebiet. Nach der sogenannten Theorie des Pleistozän-Bogens (Prado \& Gibbs 1993, Prado 2000) sind die heutigen Neotropischen Saisontrockenwälder als Reliktvorkommen einer mehr oder weniger kontinuierlichen Verbreitung während des Pleistozäns zu verstehen. Die Klimaveränderungen während des Pleistozäns waren nach Caetano \& Naciri (2011) in Südamerika nicht so stark wie auf den Kontinenten der Nordhalbkugel, weshalb die Vereisung nicht die Tiefebenen erreicht hat. Es wird daher angenommen, dass es Substitutionen zwischen den Vegetationstypen (wie z.B. Regenwälder und Trockenvegetation) gegeben hat. Der sogenannte Pleistozän-Bogen reicht laut Prado \& Gibbs (1993) von den Caatingas (Caatingas-Kerngebiet) im Nordosten Brasilien über den Südosten Brasiliens bis zum Zusammenfluss der Flüsse Paraguay und Paraná (Misiones-Kerngebiet), dem Südwesten Boliviens und Nordwesten Argentiniens (Subanden Piemont-Kerngebiet) und erstreckt sich sporadisch in den trockenen Andentälern Perus und der Westküste Ecuadors. Laut Oakley \& Prado (2011) sind diese Wälder in Paraguay hauptsächlich durch das „Misiones-Kerngebiet“ in Ostparaguay und teilweise im feuchten Chaco vertreten. Phyllostylon rhamnoides, Geoffroea spinosa, Cordia americana und Pterogyne nitens sind typische Baumarten der neotropischen Saisontrockenwälder (Oakley \& Prado 2011), die im Untersuchungsgebiet gefunden wurden. In dieser Arbeit wurden auch krautige und strauchige Arten dieser Florenregion zugeordnet. Einige dieser Arten sind Aechmea distichantha var. schlumbergeri, Tournefortia rubicunda, Schaefferia argentinensis und Achatocarpus praecox. An den salzbeeinflussten Standorten wurden eine Gattung und zehn Arten mit amphitropischer Verbreitung gefunden. Amphitropische Taxa sind nach Raven (1963) disjunkt in subtropischen und gemäßigten Gebieten (meist Trockengebiete) in Nord- und Südamerika verbreitet, kommen jedoch in den tropischen Gebieten dazwischen nicht vor. Von Moreira-Muñoz (2007) wurde daher der Begriff “antitropisch” verwendet. Verschiedene Autoren haben sich mit Gründen dieser disjunkten Verbreitung beschäftigt (wie z.B. Johnston 1940, Raven 1963, Solbrig 1972, Werger 1973, Carlquist 1983 und Moore et al. 2006). Die wohl anerkanntesten Theorien sind:

a) Fernverbreitung: disjunkte Verbreitung durch Zugvögel (Raven 1963, Werger 1973, Carlquist 1983). Im Fall einiger Ruellia-Arten, wie z.B. Ruellia simplex und $R$. erythropus, könnte laut Escurra (1993) aufgrund der Sameneigenschaften die Epizoochorie durch Zugvögel zu der Verbreitung geführt haben.

b) Migration: Verbreitung der Urpopulationen durch die Tropen über die Trockengebiete entlang der pazifischen Küste oder der Anden (Werger 1973, Carlquist 1983). Cressa truxillensis kommt nach Austin (2000) hauptsächlich in salzhaltigen Standorten im Süden der USA und Mexiko und im Zentrum Argentiniens vor. Einzelne Aufsammlungen entlang der Anden (Ecuador, Peru und Chile) könnten aber eine mögliche Migration durch diese Trockengebiete erklären.

c) Polytopischer Ursprung: parallele Artbildung sehr ähnlicher an Trockenheit angepasster Taxa von tropisch kontinuierlich verbreiteter Vorfahren (Johnston 1940, Werger 1973). 
Die Gattung Trichloris ist in den subtropisch-gemäßtigen Trockengebieten Nord- und Südamerikas beheimatet und wurde aufgrund ihrer amphitropischen Verbreitung von Burkart (1969) auch als „bizentrisch“ bezeichnet. Die ebenfalls amphitropisch verbreitete Trichloris crinita ist sowohl trocken- als auch salztolerant.

Insgesamt wird die floristische Zusammensetzung der untersuchten Salzstandorte sehr stark von der phytogeographischen Florenprovinz geprägt, da die gesamte Chacovegetation sehr eng mit der Alkalinität, Salinität und Textur des Bodens verbunden ist. Der größte Unterschied zur Flora des paraguayischen Chaco sind die azonalen Vegetationseinheiten, insbesondere der Salzmarschen, die hauptsächlich auf Gattungsebene große Ähnlichkeiten mit anderen tropischen und extratropischen Salzstandorten aufweisen. 


\section{Klassifikation und ökologische Charakterisierung der Salzmarschen im Chaco von Paraguay}

\subsection{Einleitung}

Die Vegetation salzhaltiger Standorte weist selbst in unterschiedlichen geographischen Regionen ähnliche Eigenschaften auf. Die azonalen Gesellschaften werden hauptsächlich von den Bodeneigenschaften und sekundär von anderen Umweltfaktoren beeinflusst (Nebbia \& Zalba 2007). Wasserverfügbarkeit und Salinität sind die bestimmenden ökologischen Faktoren bei der Verbreitung halophiler Arten und Gesellschaften (Cantero 1999, Kataria 2008). Diskontinuitäten in der Vegetation entlang eines Gradienten hängen nach Cantero et al. (1998b) größtenteils von der Tiefe und Salinität des Grundwassers ab, welche wiederum die Bodensalinität kontrollieren. Komplexe Interaktionen zwischen Hydromorphie und Halomorphie bestimmen daher die Verbindungen zwischen salzhaltigen Standorten und den jeweiligen floristischen Gruppen.

Halophyten können laut Le Houérou (1993) nach der Wasserverfügbarkeit im Boden in Xerohalophyten (hohe Salz- und Trockentoleranz), Mesohalophyten (mäßig trockentolerant), Hygrohalophyten (dulden keine Bodentrockenheit), Phreatohalophyten (wurzeln im salzhaltigen Grundwasser), Sprayhalophyten (sind mit Küsten verknüpft) und Hydrohalophyten (salztolerante Wasserpflanzen) klassifiziert werden. Bezüglich der Textur und Dichte des Bodens kann nach dem gleichen Autor folgende Unterscheidung gemacht werden: Psammohalophyten (in sandigen Salzböden), Siltohalophyten (in schluffigen Salzböden), Pelohalopyhten (in tonigen Salzböden), Petrohalopyhten (auf steinigen salzhaltigen Substraten) und Chasmohalopyhten (in salzhaltigen Steinspalten).

Im Chaco Boreal wird nach Mereles (2005) die Vegetation in der Nähe salzhaltiger Einschwemmsenken und Lagunen vom wechselnden Salzgehalt im Boden beeinflusst. Entlang des Salzgradienten verändert sich die Vegetation von Salzfluren und niedrigen Halophytengebüschen in der Nähe der Niederungen bis hin zum typischen xerophilen Trockenwald. Die krautige und holzige Halophytenvegetation im paraguayischen Chaco wird von den meisten Autoren in eine Vegetationsformation zusammengefasst. Spichiger et al. (1991) definieren die „Estepas halófitas“ als azonale Einheiten und edaphische Formationen auf salzigen und trockenen Böden. Laut Huespe et al. (1991) hat der „Matorral de salinar“ im Chaco Boreal eine Ausdehnung von 272.473 ha. Mereles (2004, 2005, 2007) bezeichnet die Halophytenvegetation im paraguayischen Chaco als „Matorral de saladar“, „Matorrales marginales de los riachos y lagunas saladas“ und „Sabanas de saladares“. In den Beschreibungen wird zwar auf die strukturelle und biologische Heterogenität dieser Formation hingewiesen, jedoch fehlte bisher eine Differenzierung nach floristischen, ökologischen und strukturellen Kriterien sowie eine pflanzensoziologische Klassifikation der einzelnen Halophytengesellschaften.

Anhand originaler pflanzensoziologischer Vegetationsaufnahmen werden in diesem Kapitel die Gesellschaften der Salzmarschen im paraguayischen Chaco beschrieben und ökologisch charakterisiert. 


\subsection{Methoden}

\subsubsection{Vegetationsaufnahmen}

In den Sommermonaten 2010 - 2012 wurden in den Salzmarschen (einzelne Untersuchungsorte im Kapitel 2.5) 162 stratifiziert-randomisierte Probeflächen von $16 \mathrm{~m}^{2}$ in physiognomisch unterscheidbarer homogener Vegetation angelegt und pflanzensoziologisch untersucht (Anhang 4-1). Der Mindestabstand einzelner Probeflächen voneinander betrug zehn Meter. In jeder Untersuchungsfläche wurde der Deckungsgrad sowie die maximale Höhe der Vegetation geschätzt. Zudem wurden die Deckung der Streuschicht und des offenen Bodens notiert. Von jeder Probefläche wurde eine Artenliste angefertigt mit genauen Angaben zur Wuchsform und Artmächtigkeit jeder Art nach der erweiterten Braun-BlanquetSkala (Braun-Blanquet 1964, Wilmanns 1998).

\subsubsection{Bodenanalysen}

In 41 Probeflächen wurden jeweils ein $40 \mathrm{~cm}$ tiefes Bodenprofil angelegt, um Bodenproben von den jeweiligen Bodenhorizonten zu entnehmen (Anhang 4-3).

Die Analysen der Bodenproben wurden im Departamento de Suelos y Ordenamiento Territorial der Fakultät für Agrarwissenschaften (Universidad Nacional de Asunción) durchgeführt. Von den Bodenproben wurden jeweils die Korngrößenverteilung, pH-Wert, Organische Substanz, Calcium-, Magnesium- und Natriumgehalt sowie die Elektrische Leitfähigkeit analysiert.

Die Bestimmung der Korngrößenverteilung erfolgte durch das Aräometer-Verfahren nach Bouyoucos (1962). Der Gehalt an organischer Substanz wurde durch die Walkley-BlackMethode (Lichterfelder Methode) mit nasser Veraschung der organischen Substanz mittels eines Gemisches aus Kaliumdichromat und Schwefelsäure ermittelt (nach Soil Survey Staff 1996).

Mittels eines $\mathrm{pH}-$ Meters wurde die Bodenreaktion (pH-Wert) potentiometrisch in destilliertem Wasser gemessen. Die elektrische Leitfähigkeit (Salinität) wurde in konstanter Temperatur von $25^{\circ} \mathrm{C}$ konduktometrisch mit einem Leitfähigkeitsmessgerät ermittelt. Die Bestimmung der Anteile wasserlöslicher Kationen (Calcium, Magnesium und Natrium) erfolgte mittels Atomabsorptionsspektrometrie.

Für die statistische Auswertung wurden die analysierten Bodenparameter der Horizonte eines jeden Profils proportional in eine jeweils $20 \mathrm{~cm}$ mächtige obere und untere Bodenschicht umgerechnet.

\subsubsection{Datenauswertung}

Die im Gelände aufgenommenen Artdaten wurden in die Vegetationsdatenbank Turboveg (Hennekens \& Schaminée 2001) eingetragen. Die Klassifikation der Vegetationseinheiten wurde mit der in JUICE (Tichý 2002) enthaltenen und nach Roleček et al. (2009) modifizierten TWINSPAN-Analyse (two-way indicator species analysis; Hill 1979) durchgeführt. Damit die TWINSPAN-Analyse eine Gewichtung von sehr abundanten Arten 
gegenüber den weniger abundanten vornehmen konnte, wurden Pseudospecies und Cut Levels gesetzt. Die 4 Cut Levels wurden auf 0, 5, 25 und 50 \% Deckungsgrad festgelegt.

Mit dem Programm CANOCO, Version 4.5 (ter Braak \& Šmilauer 2002) wurde mittels Ordinationen die Klassifikation der Vegetationseinheiten überprüft und eventuelle Zusammenhänge zwischen der Artenzusammensetzung bzw. den Vegetationsaufnahmen und den standörtlichen Parametern untersucht.

Um herauszufinden, wie hoch die Variabilität und Heterogenität in der Artenzusammensetzung der Vegetationsaufnahmen ist, wurden Korrespondenzanalysen (DCA - Detrended Correspondence Analysis, Hill \& Gauch 1980) durchgeführt. Dabei wurden seltene Arten abgewichtet und eine log-Transformierung vorgenommen. Bei Datensätzen mit einer Gradientenlänge $\leq 3$ wurde eine Hauptkomponentenanalyse (PCA principal component analysis) verwendet.

Um bei der direkten Gradientenanalyse (CCA - Canonical Correspondence Analysis, ter Braak 1987) herauszufinden, wie signifikant die Bodenparameter mit der Artenzusammensetzung in den Aufnahmen in Verbindung standen, wurde ein unbeschränkter Monte-Carlo-Permutationstest mit 999 Permutationen durchgeführt. Die Parameter wurden schrittweise reduziert und auf ihren Zusammenhang mit der Artenverteilung getestet. Wie Leyer \& Wesche (2007) vorschlagen, wurden dabei nur diejenigen in das Regressionsmodell einbezogen, die einen signifikanten Beitrag $(\mathrm{p} \leq 0,05)$ zur Erklärung der Artenverteilung lieferten.

Die univariat-statistischen Analysen wurden mit den Programmen Excel und $R$ durchgeführt.

\subsection{Ergebnisse}

\subsubsection{Klassifikation und Beschreibung der Gesellschaften}

Großflächige krautige Halophytenfluren kommen im Untersuchungsgebiet an den Ufern von Salzpfannen und -lagunen und in salzhaltigen periodisch überfluteten Niederungen vor. Periodische langandauernde Überschwemmungen und hohe Salzgehalte im Boden verhindern die Etablierung von Gehölzen und tragen außerdem zur Ausdehnung der Halophytenfluren bei.

An den Salzstellen wurden anhand originaler Vegetationsaufnahmen neun Vegetationeinheiten unterschieden. In den meisten kommt nur eine Pflanzenart zur Vorherrschaft. Die differenzierten Gesellschaften kommen entlang eines Gradienten unterschiedlicher Wasserverfügbarkeit vor. Der Gradient reicht von der hydrohalophilen Ruppia maritima-Gesellschaft (Einheit 1, Abb. 4-1) und hygrophilen Setaria geminataGesellschaft schwach salziger Standorte (Einheit 2) bis zur stark salz- und trockenresistenten Heterostachys ritteriana Gesellschaft (Einheit 9). Entlang dieses Gradienten nimmt der Gesamtdeckungsgrad der jeweiligen Vegetationseinheiten ab und der Anteil an offenem Boden zu. Die Gesamtartenanzahl und die mittlere Artenanzahl pro Aufnahmefläche der jeweiligen Vegetationseinheiten sind in den Übergangs- und Pioniergesellschaften am höchsten. In synoptischen Tabellen (Tabelle 4-1 und Anhang 4-2) wird die Artenzusammensetzung der Vegetationseinheiten entlang dieses Gradienten dargestellt. 
Tab. 4-1: Synoptische Tabelle der krautigen Vegetationseinheiten mit Stetigkeitsangaben in \%. Die Deckungsgrade und die Höhe der Krautschicht werden jeweils mit dem Mittelwert und der Standardabweichung angegeben. Vegetationseinheiten: 1) Ruppia maritima-Gesellschaft, 2) Setaria geminata-Gesellschaften, 3) Cyperus corymbosus-Gesellschaft, 4) Cynodon niemfuensis-Gesellschaft, 5) Leptochloa fusca subsp. uninervia-Gesellschaft, 6) Sarcocornia ambigua-Gesellschaft, 7) Spartina spartinae-Gesellschaft, 8) Sesuvium portulacastrum-Sporobolus pyramidatus-Gesellschaft und 9) Heterostachys ritteriana-Gesellschaft.

\begin{tabular}{|c|c|c|c|c|c|c|c|c|c|}
\hline Vegetationseinheit & 1 & 2 & 3 & 4 & 5 & 6 & 7 & 8 & 9 \\
\hline Anzahl Aufnahmeflächen & 3 & 10 & 3 & 12 & 22 & 28 & 3 & 53 & 28 \\
\hline Mittl. Artenzahl/Aufnahmefl. & 1.0 & 2.4 & 1.3 & 3.1 & 2.7 & 2.6 & 1.7 & 3.9 & 1.8 \\
\hline Gesamtartenzahl & 1 & 12 & 2 & 10 & 13 & 14 & 2 & 28 & 8 \\
\hline \multirow[t]{2}{*}{ Gesamtdeckungsgrad (\%) } & 70.0 & 87.4 & 87.7 & 86.3 & 79.3 & 62.9 & 80.0 & 62.7 & 49.5 \\
\hline & \pm 27.8 & \pm 9.3 & \pm 9.3 & \pm 10.2 & \pm 16.4 & \pm 19.8 & \pm 5.0 & \pm 19.6 & \pm 16.0 \\
\hline \multirow[t]{2}{*}{ Streuschicht-Deckungsgrad (\%) } & 0.0 & 29.0 & 38.3 & 30.4 & 5.9 & 9.8 & 30.0 & 5.3 & 4.5 \\
\hline & & \pm 8.8 & \pm 12.6 & \pm 12.2 & \pm 8.5 & \pm 9.7 & \pm 10.0 & \pm 6.1 & \pm 7.4 \\
\hline \multirow[t]{2}{*}{ Offener Boden (\%) } & 1.7 & 8.1 & 21.7 & 9.9 & 33.0 & 40.9 & 21.7 & 48.3 & 55.2 \\
\hline & \pm 2.9 & \pm 9.4 & \pm 7.6 & \pm 11.9 & \pm 23.2 & \pm 20.9 & \pm 2.9 & \pm 22.7 & \pm 18.0 \\
\hline \multirow{2}{*}{ Max. Krautschichthöhe (m) } & 0.1 & 0.4 & 0.5 & 0.6 & 0.6 & 0.5 & 1.3 & 0.4 & 0.6 \\
\hline & \pm 0.2 & \pm 0.1 & \pm 0.3 & \pm 0.4 & \pm 0.2 & \pm 0.2 & \pm 0.2 & \pm 0.1 & \pm 0.3 \\
\hline Ruppia maritima & 100 & & & & & & & & \\
\hline Setaria geminata & & 96 & & & & & & & \\
\hline Eleocharis elegans & & 43 & & & & & & & \\
\hline Euphorbia serpens & & 34 & & & 3 & & & 1 & \\
\hline Heteranthera multiflora & & 30 & & & & & & & \\
\hline Paspalum sp. & & 30 & & & & & & & \\
\hline Euphorbia lasiocarpa & & 24 & & & & & & 7 & \\
\hline Eclipta prostrata & & 27 & & & & & & & \\
\hline Spilanthes paraguayensis & & 24 & & & 8 & & & & \\
\hline Ruellia simplex & & 25 & & & & 6 & & & \\
\hline Neptunia pubescens & & 18 & & & 16 & & & & \\
\hline Cyperus corymbosus var. subnodosus & & & 100 & & & & & & \\
\hline Cynodon niemfuensis & & & & 97 & & & & & \\
\hline Parkinsonia aculeata (juv) & & & & 42 & & & & 4 & \\
\hline Leptochloa virgata & & & & 27 & & & & & \\
\hline Leptochloa fusca subsp. uninervia & & & & 20 & 60 & 8 & & 5 & \\
\hline Cressa truxillensis & & & & 14 & 22 & 11 & & 1 & \\
\hline Acacia praecox & & & & & 20 & & & & \\
\hline Sesbania virgata & & & & & 29 & & & & \\
\hline Tessaria dodonaeifolia & & & & & 20 & & & & \\
\hline Sarcocornia ambigua & & & & & & 55 & 29 & 1 & \\
\hline Spartina spartinae & & & & & & & 100 & & \\
\hline Commelina erecta & & & & & & 18 & & & \\
\hline Coleataenia prionitis & & & & & & 18 & & & \\
\hline Trichloris crinita & & & & & & 14 & & 6 & \\
\hline Sporobolus pyramidatus & & & & & 14 & 11 & & 54 & 1 \\
\hline Sesuvium portulacastrum & & & & 13 & 13 & 7 & & 46 & \\
\hline Heliotropium curassavicum & & & & 4 & & 7 & & 36 & \\
\hline Portulaca cryptopetala & & & & & & 4 & & 32 & \\
\hline Prosopis ruscifolia (juv) & & & & 19 & & 4 & & 26 & \\
\hline Solanum multispinum & & & & & & & & 18 & \\
\hline Atriplex eximia & & & & & & & & 18 & \\
\hline Pterocaulon purpurascens & & & & & & & & 32 & \\
\hline Portulaca oleracea & & & & & & 9 & & 16 & \\
\hline Funastrum flavum & & & & & & & & 18 & \\
\hline Pappophorum krapovickasii & & & & & & & & 26 & \\
\hline Lycium americanum & & & & & & & & 13 & \\
\hline Urochloa adspersa & & & & & & & & 13 & \\
\hline Sclerophylax spinescens & & & & & & & & 13 & \\
\hline Heterostachys ritteriana & & & & & & & & & 95 \\
\hline Portulaca grandiflora & & & & & & & & 19 & 28 \\
\hline Alternanthera nodifera & & & & & & & & & 25 \\
\hline Echinopsis rhodotricha & & & & & & & & & 18 \\
\hline
\end{tabular}


Die Abbildung 4-3 zeigt die am häufigsten auftretenden halophilen Krautgesellschaften in einer DCA-Ordination. Der Gradient auf der ersten Achse wird von der Wasserverfügbarkeit und der Salinität im Boden bestimmt und auf der zweiten Achse ist die Bodenart bzw. das Relief ausschlaggebend für die Verteilung der Gesellschaften. Der Leptochloa-SarcocorniaSesuvium-Komplex wird in der Abbildung 4-4 dargestellt. Die Verteilung auf der ersten Achse wird vom Salzgradienten (bzw. Natriumgehalt) bestimmt. Die zweite Achse zeigt einen Feuchtigkeitsgradienten von der hygrophilen Leptochloa fusca-Gesellschaft zur psammophilen Sesuvium portulacastrum-Sporobolus pyramidatus-Gesellschaft auf etwas höher gelegenen Standorten.

(1) Ruppia maritima-Gesellschaft: Diese monospezifische wurde im Untersuchungsgebiet nach einer langandauernden Überschwemmung im flachen $0,5 \mathrm{~m}$ tiefen Salzwasser (Elek. Leit. $=37$ $\mathrm{mS} / \mathrm{cm}$ ) gefunden. In vorangegangenen Feldarbeiten wurden nur die fadenförmigen Reste dieser Pflanzen in den ausgetrockneten Flussläufen des Riacho Yakaré Sur gefunden. Ruppia maritima (Abb. 4-1) ist eine submerse Wasserpflanze, die im brackigen oder salzigen Wasser wächst und mit einem feinen Wurzselsystem im Grund verwurzelt ist.

(2) Setaria geminata-Gesellschaft: In tiefergelegenen, periodisch überschwemmten und feuchten Zonen befindet sich die $30 \mathrm{~cm}$ hohe Grasflur. Mit einem mittleren Deckungsgrad von $87 \%$ bildet Setaria geminata häufig dichte Reinbestände oder wird von einigen wenigen Arten wie Eleocharis elegans oder Euphorbia serpens begleitet. Diese Grasflur kommt in schwach salzigen, mäßig sauren und sandig-tonigen Lehmböden vor und ist im Untersuchungsgebiet meist in Gräben oder kleinen Niederungen

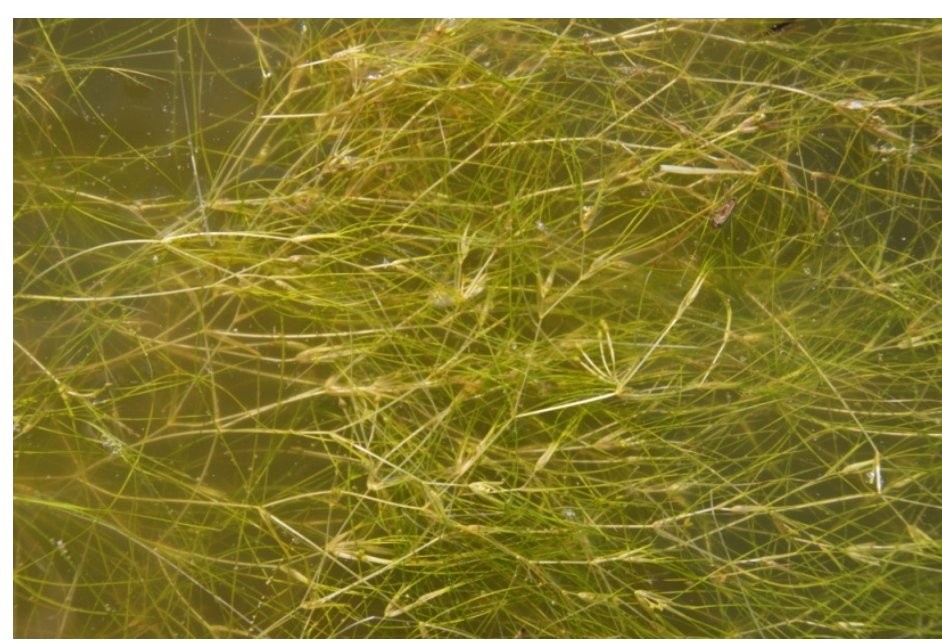

Abb. 4-1: Ruppia maritima im Riacho Yakaré Sur.

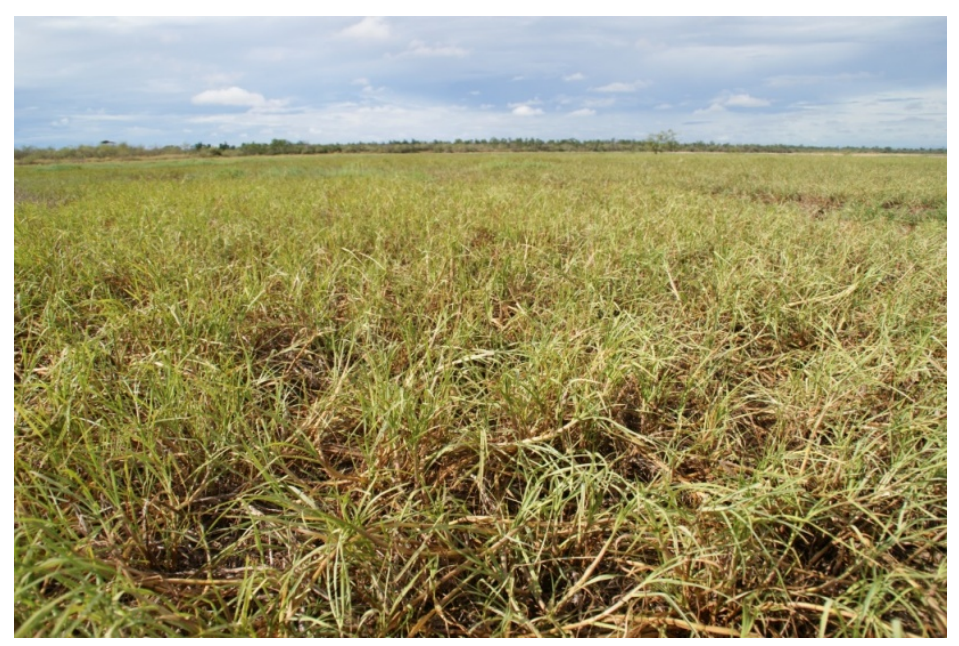

Abb. 4-2: Setaria geminata-Gesellschaft im Naturreservat Chaco Lodge. anzutreffen (Abb. 4-2). 


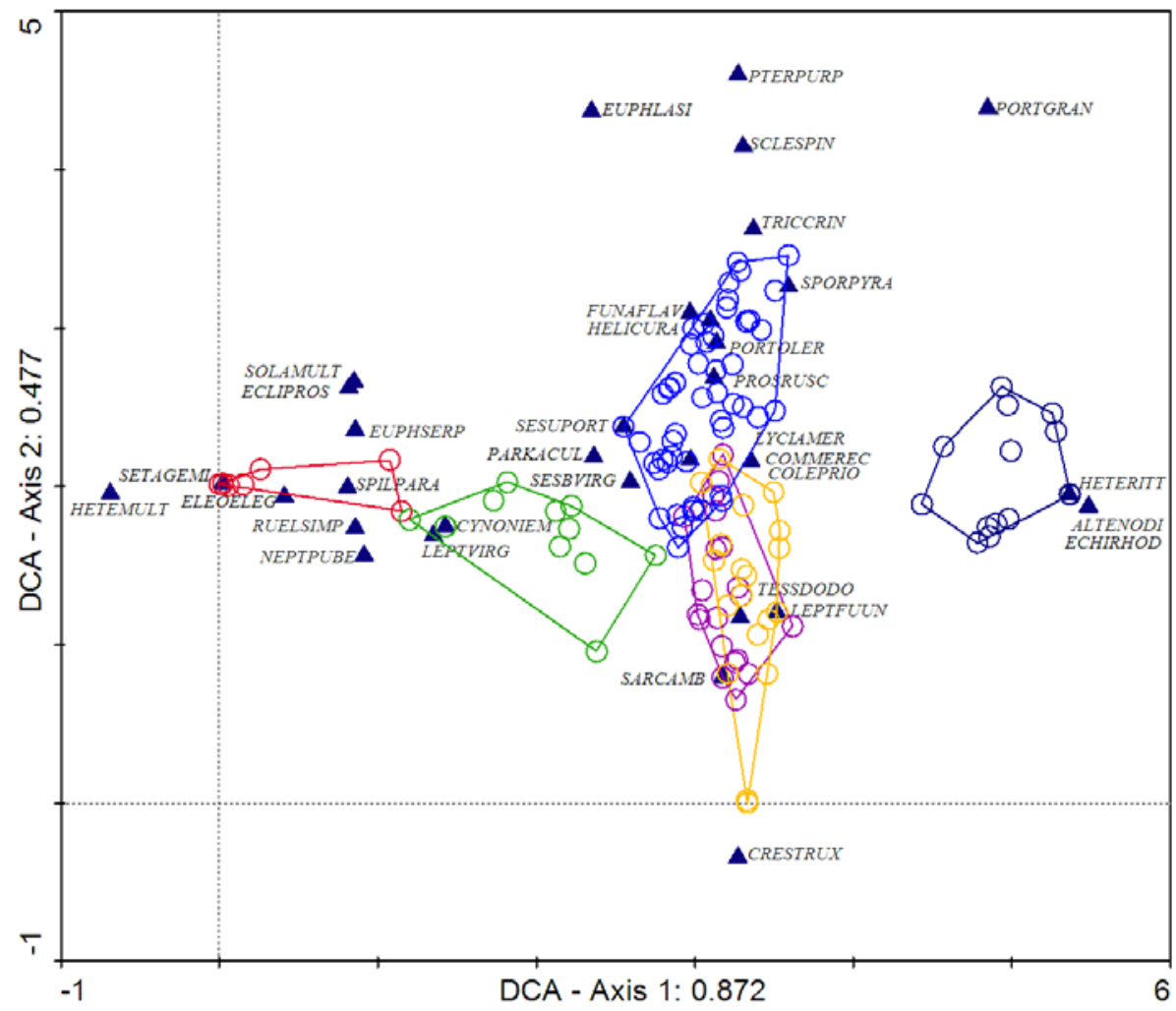

Abb. 4-3: DCA - Ordinationsdiagramm der krautigen Halophytengesellschaften: Länge des Gradienten auf der ersten Achse: 5,369 (Log-file-Daten im Anhang 4-4). Abgebildet werden 34 von den 42 Arten und die Vegetationsaufnahmen (insgesamt 153) der jeweiligen Pflanzengesellschaften: $\bigcirc$ Einheit 2, $\bigcirc$ Einheit 4, Einheit 5, $\bigcirc$ Einheit 6, $\bigcirc$ Einheit 8, $\bigcirc$ Einheit 9, $\Delta$ zeigt die Position der jeweiligen Arten (Artnamen zu den jeweiligen Abkürzungen im Anhang 4-2).

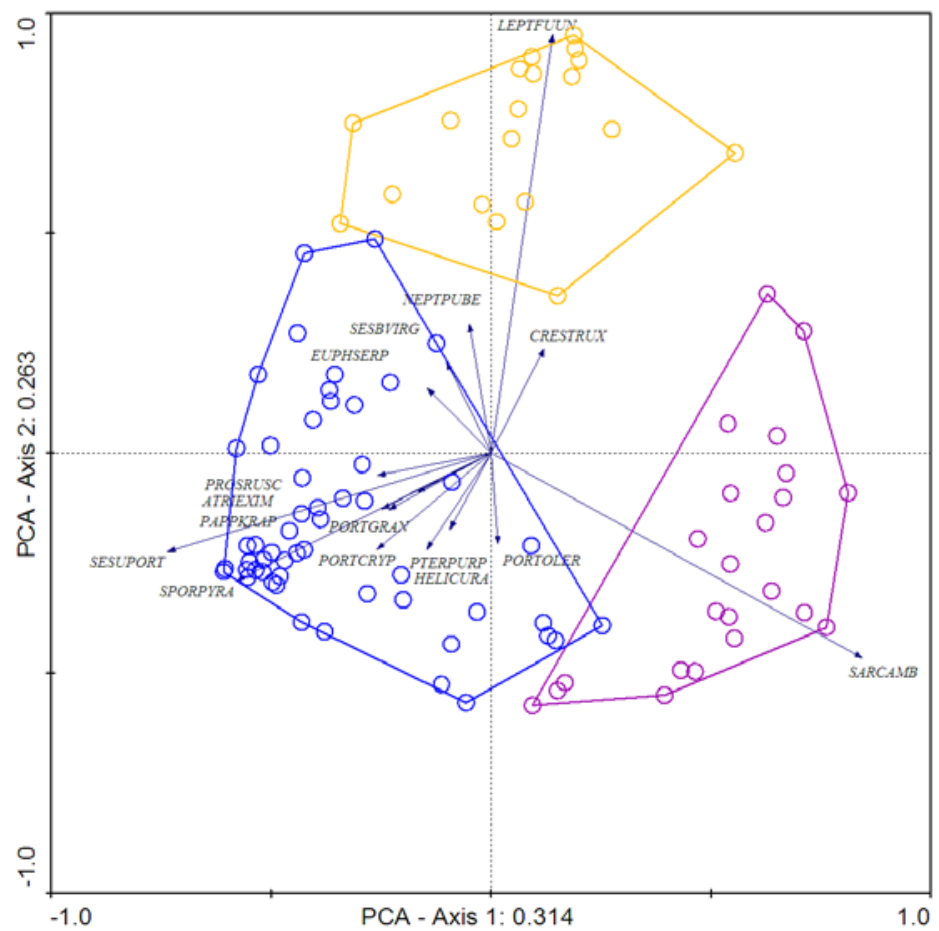

Abb. 4-4: PCA - Ordinationsdiagramm des Leptochloa-Sarcocornia-Sesuvium Komplexes. Abgebildet werden die häufigsten Arten und die Vegetationsaufnahmen (insgesamt 103) der jeweiligen Pflanzengesellschaften: $\bigcirc$ Einheit 5, $\bigcirc$ Einheit 6, $\bigcirc$ Einheit 8, $\rightarrow$ zeigt die Position der jeweiligen Arten (Artnamen zu den jeweiligen Abkürzungen im Anhang 4-2; Log-file-Daten im Anhang 4-5) 


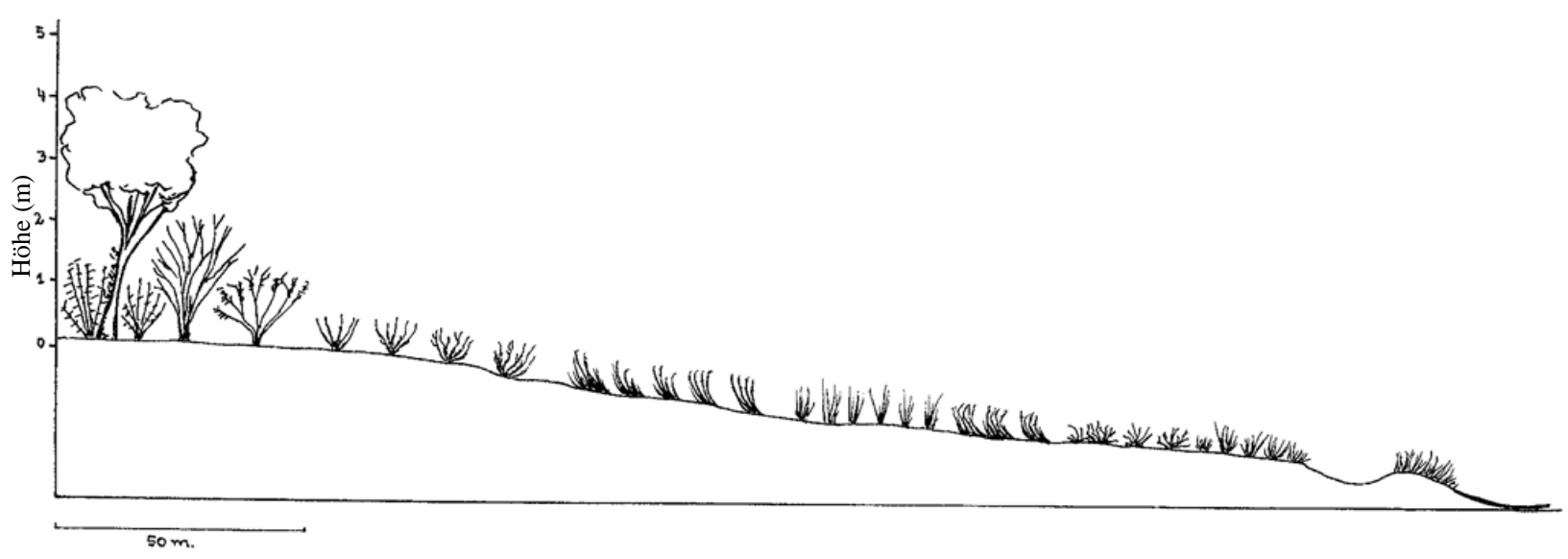

Abb. 4-5: Vegetationsprofil am Ufer einer Salzlagune. Abgebildet werden halophile Gesellschaften der Salzmarschen: 2) Setaria geminata-Gesellschaft, 4) Cynodon niemfuensis-Gesellschaft, 5) Leptochloa fusca subsp. uninervia-Gesellschaft, 6) Sarcocornia ambigua-Gesellschaft und 9) Heterostachys ritterianaGesellschaft. Bei der angrenzenden Gehölzgesellschaft handelt es sich um die Lycium americanum-Cyclolepis genistoides-Gesellschaft (Lyc.-Cyc.-Gesell.; Vegetationseinheit 14, Beschreibung in Kapitel 5.3.1.).

(3) Cyperus corymbosus-Gesellschaft: ist eine hygrohalophile Gesellschaft, die vom Rhizomgeophyt Cyperus corymbosus charakterisiert wird. Diese Art kommt in Reinbeständen vor oder wird von Sesuvium portulacastrum begleitet. Am Riacho González wurde diese Gesellschaft in kleinen Beständen in tiefergelegenen und häufig überschwemmten Standorten gefunden. Umringt wird diese Gesellschaft von der an etwas höher gelegenen Stellen wachsenden Spartina spartinae-Gesellschaft.

(4) Cynodon niemfuensis-Gesellschaft: ist eine salztolerante Grasflur, in der die Differentialart von Leptochloa fusca ssp. uninervia, Cressa truxillensis, Sesuvium portulacastrum und Jungpflanzen von Prosopis ruscifolia und Parkinsonia aculeata begleitet wird. Mit maximal $40 \mathrm{~cm}$ Höhe erreicht diese Grasflur mittlere Deckungsgrade von 86\%.

C. niemfuensis ist eine aus Afrika stammende Grasart, die weltweit als Weidegras angepflanzt wird. Im Untersuchungsgebiet findet man diese Gesellschaft auf kleinen Flächen, in periodisch überschwemmten Zonen am Rand der Lagunen und Flussläufe. Diese Einheit steht räumlich meist im engen Kontakt mit der Sarcocornia ambigua-Gesellschaft (Abb. 4-5).

Die Vegetation an den Ufern der Lagunen und häufig überschwemmten Niederungen (Abb. 45) wird größtenteils von hygrohalophilen Gesellschaften geprägt. Langandauernde Überschwemmungen und stark wechselnder Salzgehalt im Boden bestimmen hier die Artenzusammensetzung.

(5) Leptochloa fusca subsp. uninervia-Gesellschaft: kleinflächige $60 \mathrm{~cm}$ hohe Pioniergrasflur in feuchten und periodisch überfluteten Zonen. Dominiert wird diese Gesellschaft von der ein- bis mehrjährigen Leptochloa fusca subsp. uninervia. In tonigen und feuchten Gräben kommt die Art in Reinbeständen vor. In sandiglehmigen Böden an den Ufern der Salzlagunen kommt nach Rückgang des Wassers zuerst der Rhizomgeophyt Cressa truxillensis vor und danach etabliert sich Leptochloa fusca. Begleitarten in niedrigen 
Deckungsgraden sind Sesuvium portulacastrum und Sporobolus pyramidatus. Räumlich steht die Gesellschaft im direkten Kontakt mit der Sarcocornia ambigua Gesellschaft.

(6) Sarcocornia ambigua-Gesellschaft: artenarme Gesellschaft, die meist in Reinbeständen vorkommt oder von einigen wenigen Begleitarten, wie Cressa truxillensis, Leptochloa fusca subsp. uninervia, Sesuvium portulacastrum oder Sporobolus pyramidatus begleitet wird. Charakteristisch für diese Zwergstrauch-Gesellschaft (Abb. 46) sind die grundwassernahen Standorte mit salzreichen und häufig überschwemmten Böden und der relativ hohe Anteil an offenem Boden (Mittelwert=41\%). Im Untersuchungsgebiet steht diese Gesellschaft räumlich im engen Kontakt mit der Heterostachys ritteriana-Gesellschaft, ist jedoch

Abb. 4-6: Sarcocornia ambigua-Gesellschaft im Naturreservat Chaco Lodge. stauwasserverträglicher als diese.

\section{(7) Spartina spartinae-Gesellschaft:} $1,3 \mathrm{~m}$ hohe Grasflur in stark salzhaltigen periodisch überfluteten Flussbetten (Abb. 4-7). Die dominierende Art kommt in Reinbeständen vor oder wird in niedrigen Deckungsgraden von Sarcocornia ambigua begleitet. Die Gesellschaft wurde nur am Riacho Gonzalez gefunden und steht räumlich im direkten Kontakt zur Heterostachys ritteriana-Gesellschaft.

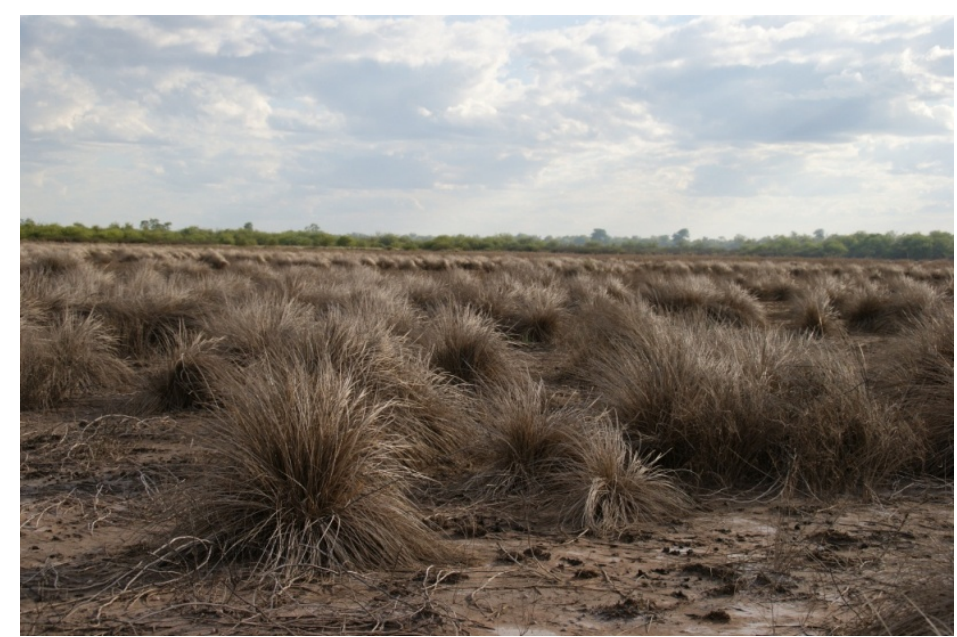

Abb. 4-7: Spartina spartinae-Gesellschaft am Riacho González.

(8) Sesuvium portulacastrum-Sporobolus pyramidatus-Gesellschaft: Pioniergesellschaft, die Sedimentaufschüttungen und gestörte Stellen besiedelt und von Sesuvium portulacastrum und Sporobolus pyramidatus dominiert wird. Weitere Differenzialarten sind Heliotropium curassavicum var. argentinum, Portulaca cryptopetala, Pterocoaulon purpurascens und Atriplex eximia.

Im Untersuchungsgebiet wurden zwei Entwicklungsstadien dieser Gesellschaft beobachtet. Sesuvium portulacastrum ist die erste Art, die diese Stellen besiedelt und übernimmt daher am Anfang die Vorherrschaft. Die nächsten Besiedler sind Sporobolus pyramidatus und Heliotropium curassavicum. 


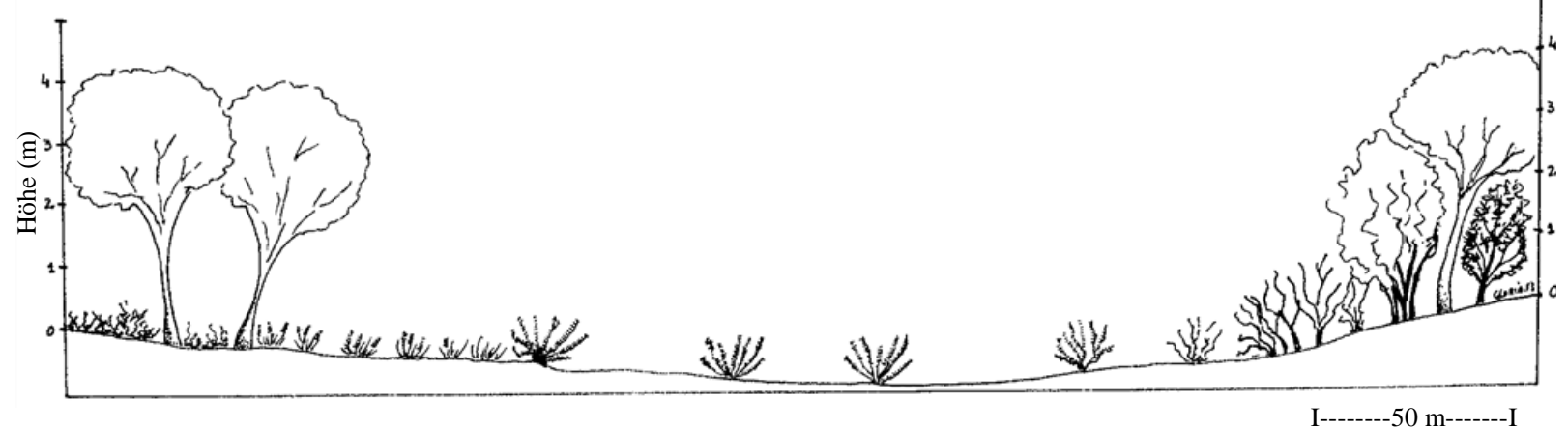

Abb. 4-8: Vegetationsprofil einer typischen Salzpfanne. Abgebildet werden zwei halophile Gesellschaften der Salzmarschen: 8) Sporobolus pyramidatus-Sesuvium portulacastrum-Gesellschaft und 9) Heterostachys ritteriana-Gesellschaft. Bei den angrenzenden Gehölzgesellschaften handelt es sich um die Prosopis ruscifoliaSporobolus pryramidatus-Gesellschaft (Pros.-Spor.-Gesell.; Einheit 11, Kapitel 5.3.1) und die Prosopis ruscifolia-Lycium americanum-Gesellschaft (Pros.-Lyc.-Gesell.; Einheit 13, Kapitel 5.3.1).

Mehrere niederschlagsarme Jahre führen zur Ansiedlung von Gehölzjungpflanzen von Prosopis ruscifolia und Parkinsonia aculeata und somit zur Verbuschung dieser Gesellschaft.

Diese Gesellschaft entwickelt sich auf stark sandig-tonigen oder stark sandiglehmigen Böden, meist auf Aufschüttungsstellen die durch Flut oder Windaufwehungen entstanden sind (Abb. 4-9).

(9) Heterostachys ritteriana-Gesellschaft: Im Untersuchungsgebiet sind Salz-pfannen häufig in flachen ausgetrockneten Flussbetten zu finden (Abb. 4-8). Im Inneren dieser Salzpfannen findet man vorwiegend die eine Zwergstrauchgesellschaft mit Heterostachys ritteriana. Die maximal $1 \mathrm{~m}$ hohe Halophytenflur fällt durch ihre große Flächenausdehnung und geringe Gesamtartenzahl auf. In den meisten Fällen sind es Reinbestände der einen Art, doch kann diese auch von einzelnen Arten wie Portulaca

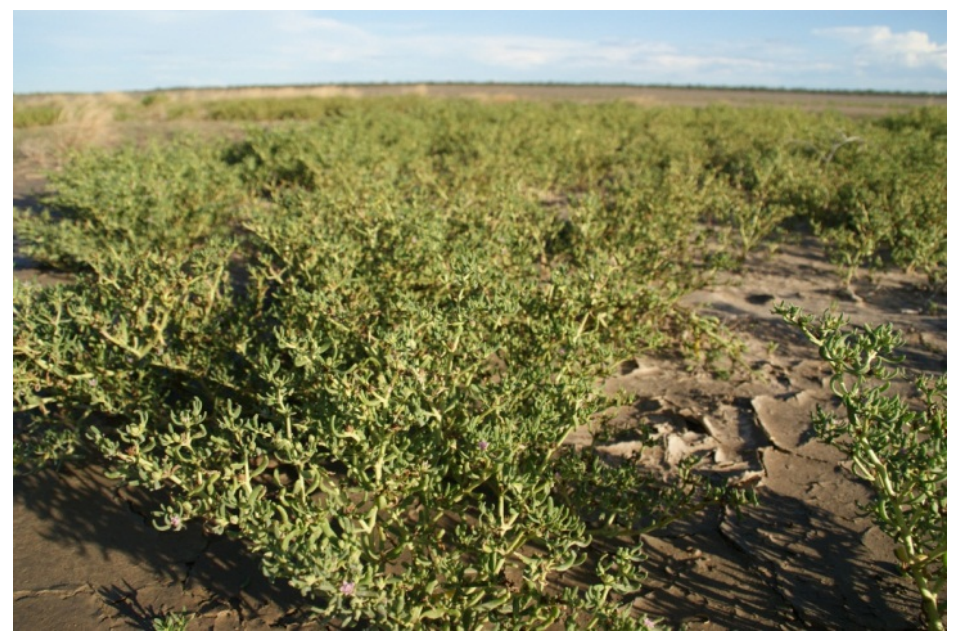

Abb. 4-9: Sesuvium portulacastrum-Sporobolus pyramidatusGesellschaft auf Sedimentaufschüttungen.

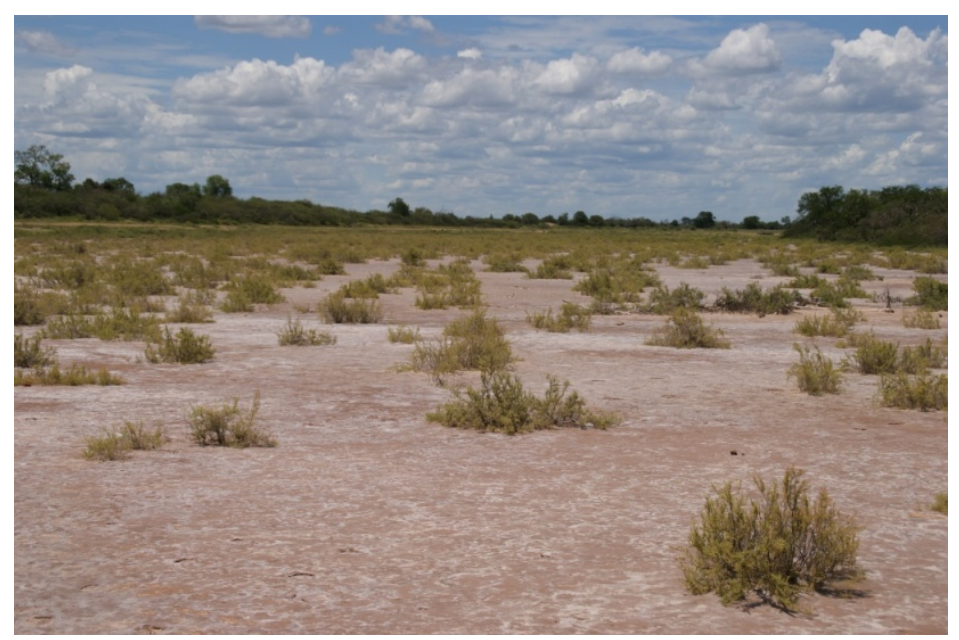

Abb. 4-10: Heterostachys ritteriana-Gesellschaft in einem salzhaltigen und ausgetrockneten Flussbett. 
grandiflora, Leptochloa fusca ssp. uninervia oder Alternanthera nodifera begleitet werden. Charakteristisch für diese Halophyten-gesellschaft sind wechseltrockene und salzreiche Standorte mit tonig-sandigen bis sandig-lehmigen und natrium-reichen Oberböden. Auffällig ist der hohe Anteil an offenem Boden (Mittelwert=55\%), der in niederschlagsarmen Jahreszeiten von einer weißen Salzkruste bedeckt sein kann (Abb. 4-10).

Die Heterostachys ritteriana-Gesellschaft ist im Untersuchungsgebiet weit verbreitet; besonders auf der Außenseite der Mäander und an den Enden der Lagunen kann sie große Flächenausdehnungen erreichen.

\subsection{2. Ökologie und Dynamik der Salzmarschen}

Da die untersuchten Salzmarschen sich in periodisch wasserführenden Flusssystemen und Einschwemmsenken befinden, werden die Oberböden von fluvialen Sedimentablagerungen bzw. Erosionen beeinflusst. Zudem führen starke Winde in den langen Trockenperioden zu beachtlichen Sedimentverlagerungen in den zum größten Teil offenen Bodenoberflächen der Salzmarschen. Aus diesen Gründen spielt wohl die Bodenart der oberen Bodenhorizonte nur eine untergeordnete Rolle in der Verbreitung der halophilen Gesellschaften. Wie in einer indirekten Gradientenanalyse (Abb. 4-12) dennoch ersichtlich ist, wachsen Setaria geminata, Cynodon niemfuensis und Leptochloa fusca subsp. uninervia größtenteils in Standorten mit höheren Tonanteilen im Oberboden, während Sesuvium portulacastrum, Sporobolus pyramidatus, Heterostachys ritteriana und Sarcocornia ambigua Standorte mit höheren Sandanteilen im Oberboden besiedeln. Die Bedeutung der Korngrößenanteile des oberen Bodenhorizontes ist dabei wesentlich höher als die des unteren.

Im Gelände wurden zwar nur schwache Reliefunterschiede an den Ufern der Lagunen und Flussbetten beobachtet, jedoch beeinflussen diese maßgeblich die Sedimentablagerungen, Wasserverfügbarkeit und Dauer der Überschwemmung. Die Sesuvium portulacastrumSporobolus pyramidatus- und die Heterostachys ritteriana-Gesellschaften besiedeln meist höher gelegene wechseltrockene Standorte und meiden wechselfeuchte Standorte, die lange überschwemmt werden. Nach extremen Niederschlägen im Jahr 2012 und monatelanger Staunässe konnte ein großflächiges Absterben der Heterostachys ritteriana-Populationen beobachtet werden. (Abb. 4-11).

Auch Sporobolus pyramidatus- und Sesuvium portulacastrum-Bestände verschwanden und erst nach Rückgang des Wassers kamen erste Keimlinge wieder vor. Da diese langandauernden

Überschwemmungen periodisch im Chaco auftreten, kann man davon ausgehen, dass dadurch die Populationen in Abständen erneuert werden.

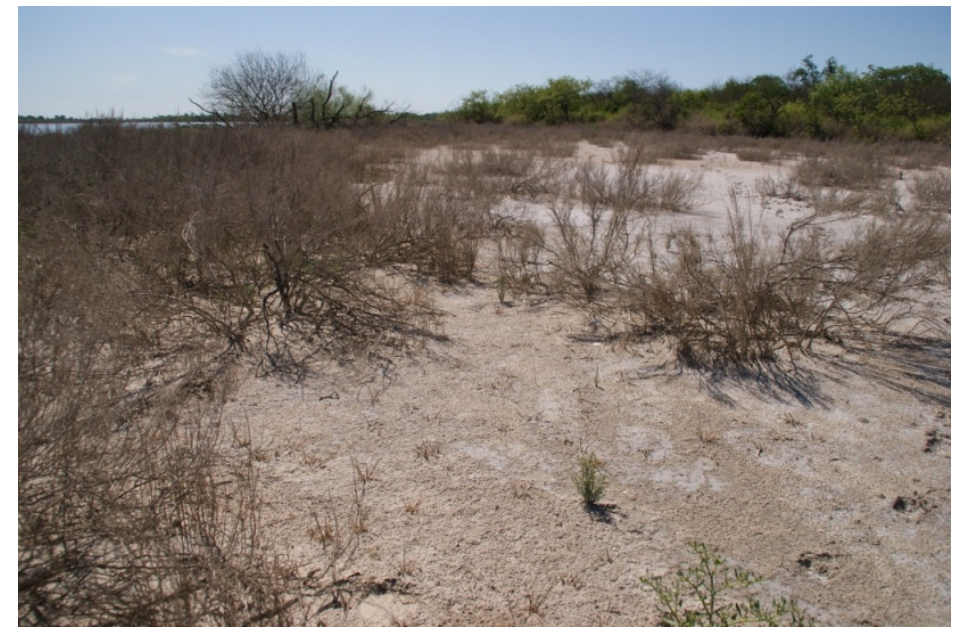

Abb. 4-11: Abgestorbene Heterostachys ritteriana-Population nach einer langanhaltenden Überschwemmung im Jahr 2012. 


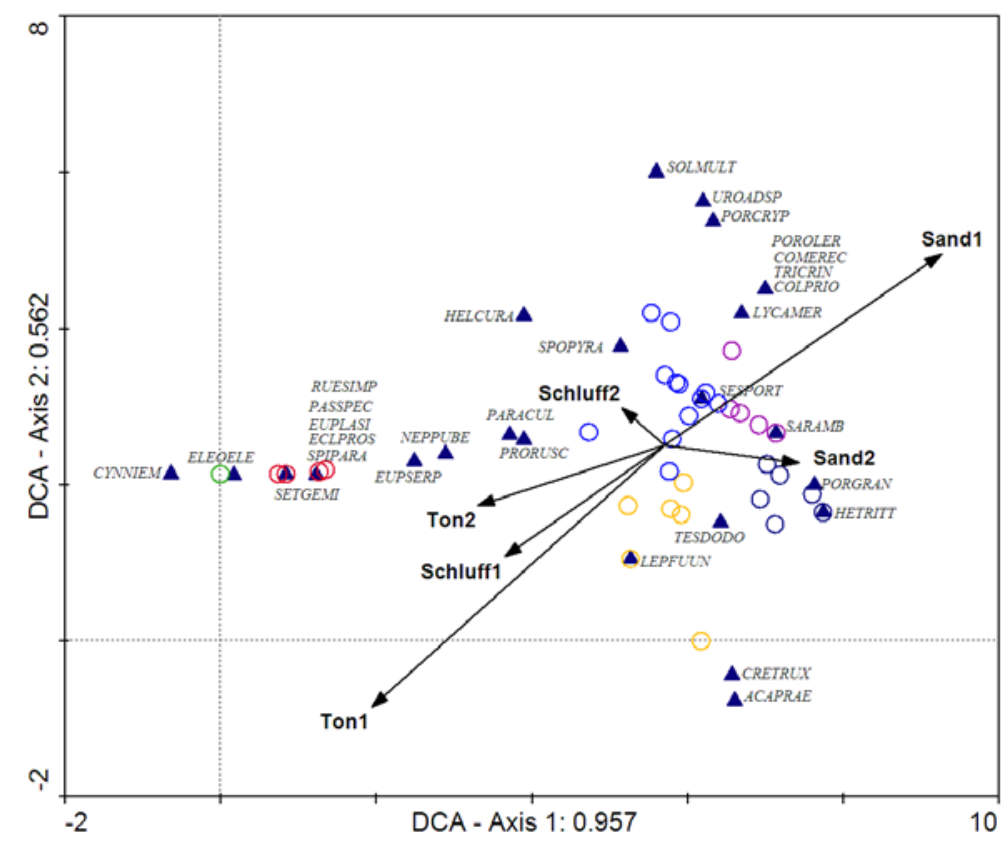

Abb. 4-12: Ordinationsdiagramm der häufigsten Salzmarsch-Gesellschaften in einer indirekten Gradientenanalyse mit den Bodenarten. Abgebildet werden die Arten (insgesamt 30), die Probeflächen (insgesamt 41) und die Bodenarten: Sand, Schluff und Ton in der oberen (1) und unteren (2) Bodenschicht. Die abgebildeten Pflanzengesellschaften sind: $\bigcirc$ Einheit 2, $\bigcirc$ Einheit 4, $\bigcirc$ Einheit 5, $\bigcirc$ Einheit 6, $\bigcirc$ Einheit 8, $\bigcirc$ Einheit 9, $\boldsymbol{\Delta}$ zeigt die Position der jeweiligen Arten (Artnamen zu den jeweilgen Abkürzungen im Anhang 4-2; Log-file-Daten im Anhang 4-6).

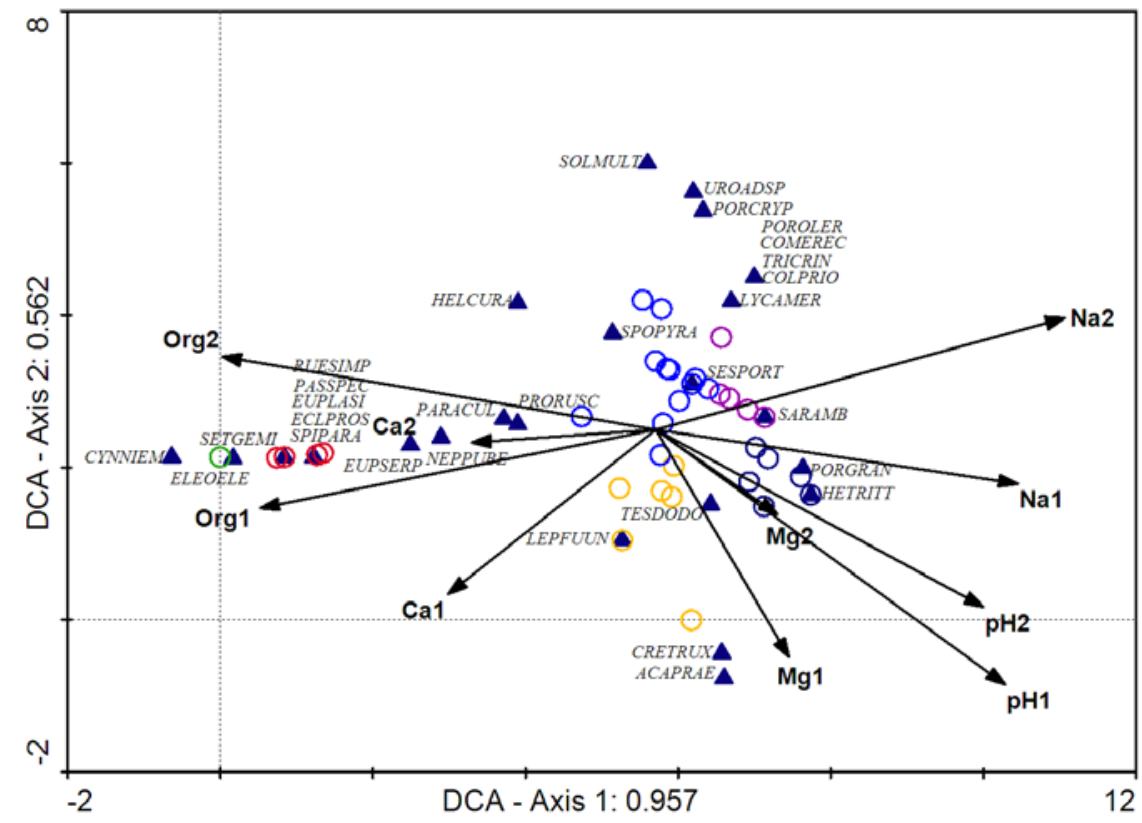

Abb. 4-13: Ordinationsdiagramm der häufigsten Salzmarsch-Gesellschaften in einer indirekten Gradientenanalyse mit den chemischen Bodenparametern. Abgebildet werden die Arten (insgesamt 30), die Probeflächen (insgesamt 41) und die Bodenparameter: pH, Natrium (Na), Magnesium (Mg), Calcium (Ca) und Organische Masse (Org) in der oberen (1) und unteren (2) Bodenschicht. Die abgebildeten Pflanzengesellschaften sind: $\bigcirc$ Einheit 2, $\bigcirc$ Einheit 4, $\bigcirc$ Einheit 5, $\bigcirc$ Einheit $6, \bigcirc$ Einheit $8, \bigcirc$ Einheit 9, $\boldsymbol{\Delta}$ zeigt die Position der jeweiligen Arten (Artnamen zu den jeweiligen Abkürzungen im Anhang $4-2)$ 


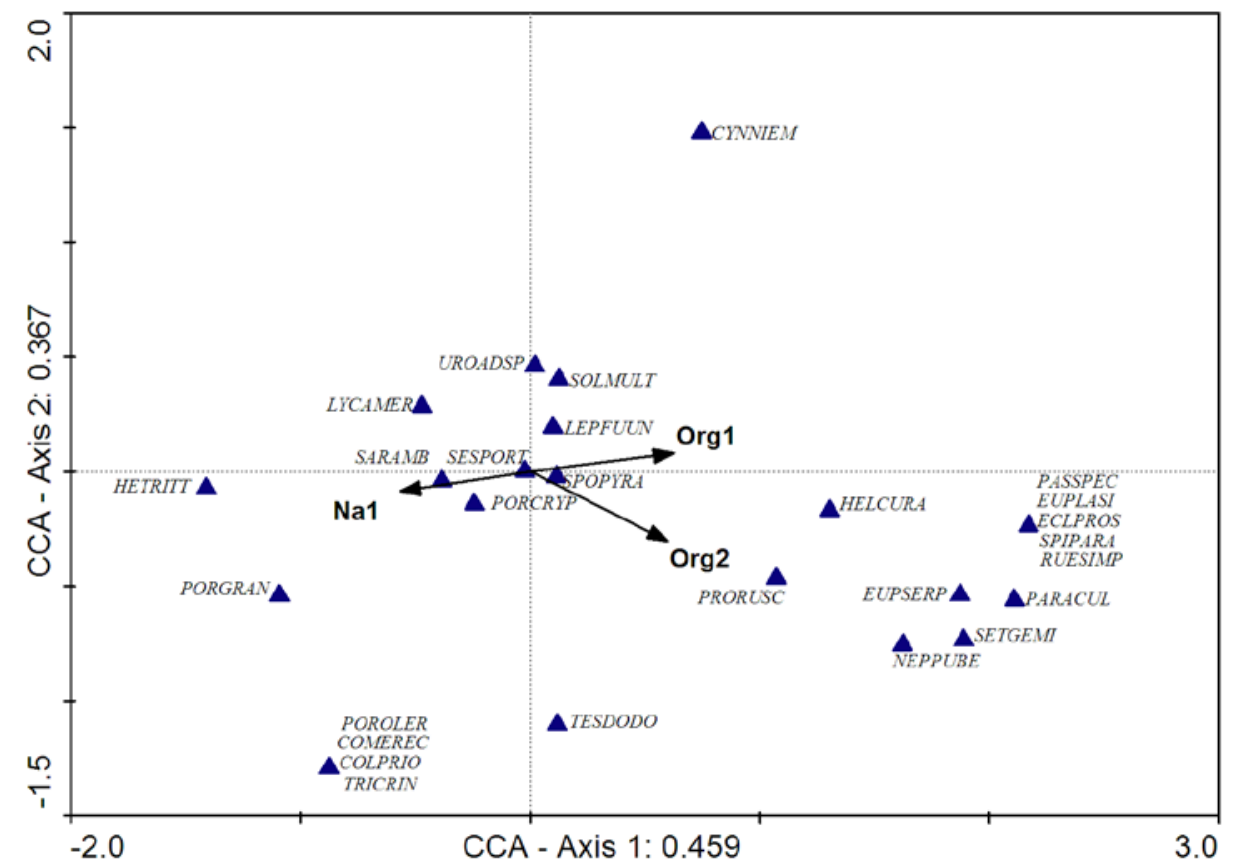

Abb. 4-14: CCA - Ordinationsdiagramm der signifkanten Bodenparameter. Abgebildet werden die Arten und die drei signifikanten Bodenparametern: organische Masse in der unteren Bodenschicht (Org2, pWert=0,01), organische Masse in der oberen Bodenschicht (Org1, p-Wert=0,03) und Natrium in der oberen Bodenschicht (Na1, p-Wert=0.046). $\mathbf{\Delta}$ zeigt die Position der jeweiligen Arten (Artnamen zu den jeweiligen Abkürzungen im Anhang 4-2; Log-file-Daten im Anhang 4-7)

Die chemische Zusammensetzung des Oberbodens kann anhand einer indirekten Gradientenanalyse (Abb. 4-13) eine annähernde Erklärung zur Verbreitung der Gesellschaften in den Salzsteppen geben. Standorte der Setaria geminata-Gesellschaft weisen einen höheren Anteil an organischer Substanz auf. Hohe Natriumgehalte im Oberboden fördern die Etablierung der Sarcocornia ambigua- und der Heterostachys ritterianaGesellschaften. Böden mit höheren Anteile an Magnesium und Calcium dagegen werden gern von der Leptochloa fusca subsp. uninervia-Gesellschaft besiedelt.

Mit einer direkten Gradientenanalyse (Abb. 4-14) konnten die organische Substanz und der Natriumgehalt im Oberboden als signifikante Bodeneigenschaften für die Verteilung der Arten festgestellt werden. Hygrophile Arten wie Setaria geminata, Neptunia pubescens und Parkinsonia aculeata kommen in Böden mit höherem Humusanteil vor, während in natriumreichen Böden Heterostachys ritteriana und Sarcocornia ambigua vorherrschen.

Die Boxplot-Analysen in Abbildung 4-15, die auf Daten der An- und Abwesenheit einiger halophiler Arten in den jeweiligen untersuchten Standorten beruhen, zeigen einen besonders großen Unterschied zwischen den Standorten mit Heterostachys ritteriana und Setaria geminata. Setaria geminata wächst in schwach sauren Standorten, während die anderen Arten alkalische Böden besiedeln. Trotz der Überlappung der Leitfähigkeitswerte im Boden können die Standorte mit Heterostachys ritteriana im Vergleich zu den anderen Arten als stark salzhaltig definiert werden. 

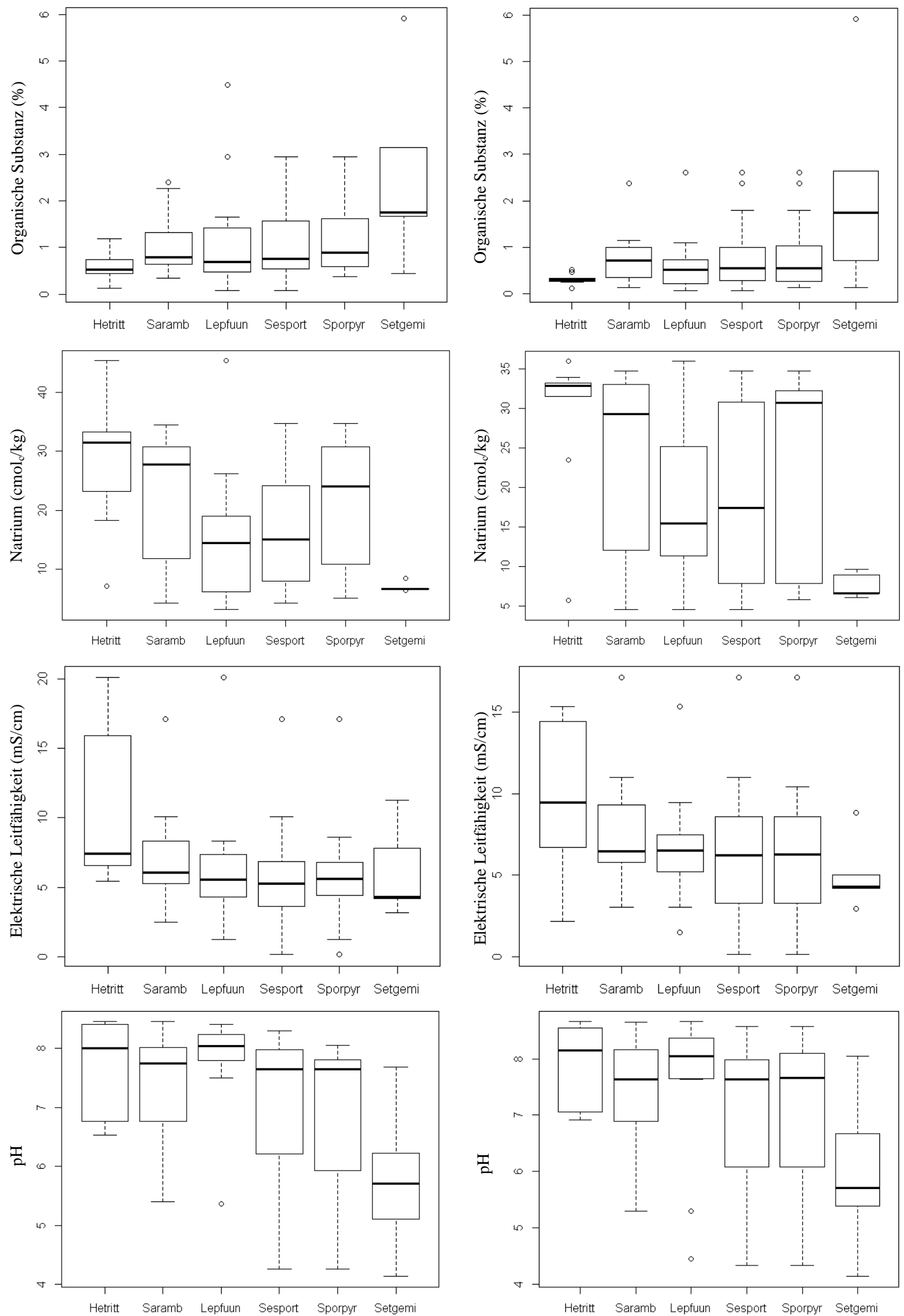

Abb. 4-15: Charakterisierung der Standorte von Heterostachys ritteriana (Hetritt), Sarcocornia ambigua (Saramb), Leptochloa fusca subsp. uninervia (Lepfuun), Sesuvium portulacastrum (Sesport), Sporobolus pyramidatus (Sporpyr) und Setaria geminata (Setgemi). Abgebildet werden Boxplots mit den jeweiligen Daten der organischen Substanz (\%), Natriumgehalt $\left(\mathrm{cmol}_{\mathrm{C}} / \mathrm{kg}\right)$, elektrische Leitfähigkeit $(\mathrm{mS} / \mathrm{cm})$ und $\mathrm{pH}$ in der oberen (links) und der unteren (rechts) Bodenschicht. Für die standörtliche Charakterisierung zählt die Anwesenheit der halophilen Arten im untersuchten Standort. 
Alle untersuchten Standorte weisen natriumbeeinflusste Böden auf. Auch hier ist wieder ein großer Unterschied zwischen den mäßig natriumhaltigen Setaria geminata-Standorten und den Heterostachys ritteriana-Standorten mit sehr hohen Natriumgehalten im Oberboden ersichtlich. Sarcocornia ambigua, Leptochloa fusca subsp. uninervia, Sesuvium portulacastrum und Sporobolus pyramidatus scheinen an sehr große Variationen des Natriumgehaltes angepasst zu sein. Der stark wechselnde Natrium- und Salzgehalt im Oberboden ist auf die grundwasserbeeinflussten Standorte zurückzuführen.

Die Böden der Salzmarschen weisen insgesamt sehr niedrige Humusgehalte auf. Hohe Salinität, Trockenheit und offene der hohen Sonneneinstrahlung ausgesetzte Bodenoberflächen hemmen die Humusbildung. Im Gegensatz dazu weisen feuchte und mäßig salzhaltige Böden der Setaria geminata-Standorte, die durch eine hohe Bodenbedeckung charakterisiert werden, höhere Humusgehalte auf.

Bei mehreren Arten in den untersuchten Salzmarschen ist die Sukkulenz eine typische Eigenschaft, um aufgenommene Salzmengen zu verdünnen. $\mathrm{Zu}$ den sukkulenten Arten gehören Heterostachys ritteriana, Sarcocornia ambigua, Sesuvium portulacastrum, Heliotropium currasavicum und mehrere Arten der Gattung Portulaca. Besonders bei Heterostachys ritteriana und Sarcocornia ambigua konnte in langandauernden Dürreperioden massive Vergilbung und Abwurf der Blätter beobachtet werden, was auf Absalzungsphänomene schließen lässt.

\subsection{Diskussion}

\subsubsection{Halophytengesellschaften im überregionalen Vergleich}

Die differenzierten Gesellschaften in den untersuchten Salzmarschen weisen sowohl physiognomische als auch taxonomische Ähnlichkeiten mit halophilen Gesellschaften auf regionaler, überregionaler und kontinentaler Ebene auf.

Hygrophile Grasfluren, in denen Setaria geminata dominiert und Leptochloa fusca subsp. uninervia einen hohen Stetigkeitswert erreicht, kommen laut Lewis et al. (1990) auch im argentinischen Chaco vor. Therburg (1997) beschreibt für die Monte-Formation auch eine kleinflächig verbreitete Cressa truxillensis-Gesellschaft. Im Untersuchungsgebiet kommen Leptochloa fusca subsp. uninervia und Cressa truxillensis meist zusammen, jedoch in unterschiedlichen phänologischen Stadien vor und wurden daher in eine Gesellschaft eingeordnet.

Die im Untersuchungsgebiet vorkommende Cyperus corymbosus-Gesellschaft wurde auch von Cantero (1999) mit ähnlichen Charakteristiken für die argentinische Pampa beschrieben. Laut Cantero (1999) stehen diese konkav geformten Geländeausschnitte aufgrund edaphischer Eigenschaften (harte Bodenschichten) meist nicht mit dem lokalen salzigen Grundwasser in Verbindung. Für das Untersuchungsgebiet fehlen diesbezüglich genauere Untersuchungen.

Sarcocornia ambigua ist nach Alonso \& Crespo (2008) vorwiegend an Salzstandorten an den amerikanischen Atlantik-Küsten verbreitet; von Kuba und den umliegenden Karibinseln über Venezuela bis nach Uruguay. Laut Faggi (1985) und Tropicos (2013) kann die Verbreitung dieser Art auch bis zum Süden Patagoniens und den Binnenlandsalzstandorten im 
Andenvorland ausgeweitet werden. Eine Sarcocornia ambigua-Gesellschaft wurde mit ähnlichen Eigenschaften auch für den Süden Patagoniens (Faggi 1985, Roig et al. 1985), der argentinischen Monte-Formation (Therburg 1997), der argentinischen Pampa (Ragonese \& Covas 1947; Cantero 1999) und der Chaco-Pampa-Ebene (Menghi et al. (2001) beschrieben. In allen genannten Gebieten wächst diese artenarme Gesellschaft meist in tonig-salzigen häufig überschwemmten Böden. Auch auf der Insel Kuba kommt nach Borhidi (1996) eine artenarme oder monospezifische Vegetationseinheit mit Sarcocornia ambigua auf salzigen und schweren Tonböden vor.

Laut Therburg (1997) ist die Heterostachys ritteriana-Gesellschaft in Mittel- und Südamerika auf stark salzhaltigen Böden sowohl im Binnenland als auch in Küstennähe weit verbreitet. Mit ähnlichen Charakteristiken wird diese Gesellschaft für die kolumbianische Halbinsel Guajira (Rieger 1976), für die nördlichen Küsten Venezuelas (Walter \& Breckle 1984), für die argentinischen Salinas Grandes (Ragonese 1951), für die Monte-Formation (Therburg 1997) und für die Pampa (Cantero 1999) beschrieben. Die Autoren verwenden auch den Begriff Heterostachys ritteriana-Gesellschaft und erwähnen, dass die Einheit vor allem durch ihre große Flächenausdehnung, ihre geringe Gesamtartenzahl sowie durch ihren sehr niedrigen Deckungsgrad auffällt. Laut Rieger (1976) besiedelt sie Standorte auf stark salzhaltigen, sandigen oder schluffigen Feinsedimenten, die stets kalkhaltig sind. Sie meidet Standorte, an denen nach Regenfällen das Wasser oberflächennah stehenbleibt.

Die Grasfluren mit Spartina spartinae sind amphitropisch verbreitet und kommen um den Golf von Mexiko und in Südamerika vor. Besonders großflächig sind diese Grasfluren in Zentral-Argentinien (Feldman et al 2009). Laut Lewis et al. (1990) und Hilgert et al. (2003) besiedelt die Spartina spartinae-Gesellschaft im argentinischen Chaco stark halomorphe Böden. Begleitarten dieser Gesellschaft sind meist Sarcocornia ambigua, Sesuvium portulacastrum und Chenopodium macrospermum.

Interessanterweise fehlen im Untersuchungsgebiet einige Gattungen der argentinischen Halophytengesellschaften. Bei diesen Gattungen, wie z.B. Frankenia, Suaeda, Allenrolfea, Puccinellia und Distichlis, handelt es sich hauptsächlich um amphitropische Gattungen mit Verbreitung in gemäßigten Klimazonen. Vermutlich sind diese floristischen Unterschiede auf die erhöhten Jahresniederschläge und Temperaturen des tropisch-subtropischen Klimas im Chaco zurückzuführen. Ähnliche phytogeographische und floristische Unterschiede in Salzstandorten wurden nach Isacch et al. (2006) auch an der Süd-West-Atlantikküste entlang eines Ariditätsgradienten unterschiedlicher Breitengrade $\left(31^{\circ} \mathrm{S}-43^{\circ} \mathrm{S}\right)$ gefunden.

\subsubsection{Syntaxonomische Einordnung der Gesellschaften}

In Anlehnung an Borhidi et al (1983), Borhidi (1996) und Galán de Mera \& Vicente (2006) können die Gesellschaften der Salzmarschen im paraguayischen Chaco syntaxonomisch in drei Klassen eingeordnet werden.

\section{- Klasse: Cladietea jamaicensis Knapp ex Borhidi 1991}

Helophytenvegetation in Sümpfen, Überschwemmungszonen und an Ufern von Flüssen im tropischen Zentral- und Südamerika.

$>$ Ordnung: Scirpo-Eleocharietalia interstinctae Borhidi \& Muñiz in Borhidi et al. 1983 
Sumpfwiesen in Verbindung mit eutrophen oder oligotrophen Süß- oder Brackgewässern.

- Verband: Sagittario-Eleocharion interstinctae Borhidi \& Del-Risco in Borhidi et al. 1983

+ Assoziation: Paspalidietum paludivagi Del-Risco \& Borhidi in Borhidi et al. 1983

Grasflur mit Setaria geminata (Syn. Paspalidium paludivagum) als vorherrschende Art, die von anderen helophilen Arten begleitet wird.

+ Cyperus corymbosus-Gesellschaft

- Klasse: Ruppietea J.Tx. 1960

$>$ Ordnung: Ruppietalia maritimae J. Tx. 1960

- Verband: Najo guadalupensis-Ruppion maritimae Galán de Mera 2006 Submerse Wasserpflanzen-Gesellschaften in salzhaltigen neotropischen Gewässern

+ Assoziation: Hadulo-Ruppietum maritimae Borhidi in Borhidi et al. 1983 (Ruppia maritima-Gesellschaft)

- Klasse: Batidi-Salicornietea (Knapp 1964) Borhidi 1991

Halophytenvegetation, die aus Blattsukkulenten-Zwergsträuchern, anuellen und ausdauernden Sukkulenten und Gräsern mit hoher osmotischer Saugspannung besteht. Die hohe Salzkonzentration im oberen Bodenhorizont verhindert die Etablierung von höherer Gehölzvegetation.

> Ordnung: Batidi-Salicornietalia ambiguae (Knapp 1964) Borhidi 1991

Neotropische Halophytenvegetation bestehend größtenteils aus sukkulenten Zwergsträuchern.

- Verband: Salicornion ambiguae Faggi 1985

+ Sarcocornia ambigua-Gesellschaft

+ Heterostachys ritteriana-Gesellschaft

$>$ Ordnung: Distichlio-Spartinetalia (Chapman 1960) Borhidi \& Del Risco in Borhidi et al 1979 (1983)

- Verband: Distichlion spicatae (Chapman 1960) Borhidi \& Del-Risco in Borhidi et al (1979) 1983

Neotropische Grasfluren mit Poaceen und Cyperaceen in salzhaltigen Standorten

+ Leptochloa fusca subsp. uninervia-Gesellschaft

+ Spartina spartinae-Gesellschaft

+ Sesuvium portulacastrum-Sporobolus pyramidatus-Gesellschaft

\subsection{3. Ökologische Eigenschaften der Salzsteppen}

Die floristischen Unterschiede salzhaltiger Standorte sind nach Cantero et al. (1998b) auf drei Faktoren zurückzuführen: a) Variationen in der chemischen Komposition und den saisonalen Dynamiken der Bodenlösungen in den oberen Bodenhorizonten, b) die Salinität und Dynamik des Grundwassers, und c) die Überflutungsdauer in der Regenperiode. Auch nach Ruiz Posse et al. (2007) wird die Anwesenheit bestimmter Pflanzenarten vom Salzgehalt, den Texturunterschieden und der Topographie in enger Verbindung mit der hydrischen und 
äolischen Dynamik der Zone bestimmt. Untersuchungen im Untersuchungsgebiet (Naturreservat Campo Maria) von Glatzle et al. (2006) zeigen, dass die Dynamik der Bodensalinität stark vom Relief, den Wetterbedinungen und der Vegetation abhängig ist. Messungen während drei Jahren in einer Salzlagune und den umliegenden Uferzonen zeigen einen drastischen Anstieg des Grundwasserspiegels und Reduzierung der Leitfähigkeit des Grundwassers nach Niederschlägen. Der mittlere Grundwasserspiegel in der Lagune lag bei 0,6 $\mathrm{m}$ und in der Uferzone bei 1,2 $\mathrm{m}$ unter der Oberfläche. Die gemessene mittlere Leitfähigkeit des Grundwassers dagegen war in der Uferzone sechs mal höher als in der Lagune.

Der pflanzenverfügbare Wassergehalt und die Salinität im Boden werden weitestgehend als die entscheidenden Umweltfaktoren für die räumliche Verteilung der Halophyten in Kontinentalsalzstellen verantwortlich gemacht (Lewis et al. 1990, Cantero 1999, Menghi et al. 2001). Laut Menghi et al. (2001) tritt der floristische Hauptgradient in zentralargentinischen hygrohalophilen Gesellschaften in Verbindung mit einem komplexen physischen Gradienten auf: von Zonen mit langanhaltender Überschwemmung bis zu höher gelegenen Zonen, die durch lange Dürre- und Salinitätsperioden charakterisiert sind. Die Salzverhältnisse allein konnten auch nach Therburg (1997) in der argentinischen Provinz Mendoza nicht für die Ausbildung der verschiedenen Halophytengesellschaften verantwortlich gemacht werden, da sich die Leitfähigkeitswerte der Böden überlappen. Auch in den Salzmarschen im Chaco Boreal sind sowohl der Salzgradient als auch die unterschiedliche Wasserverfügbarkeit im Boden für die Artenzusammensetzung verantwortlich.

Bezüglich der von Le Houérou (1993) vorgeschlagenen Klassifikation der Halophyten nach der Wasserverfügbarkeit, gehört die Ruppia maritima-Gesellschaft zur hydrohalophytischen Vegetation und die Sesuvium portulacastrum-Sporobolus pyramidatus- und die Heterostachys ritteriana-Gesellschaften $\mathrm{zu}$ den xerohalophytischen. Die restlichen differenzierten Gesellschaften können den hygro- bzw. den mesohalophytischen Standorten zugeordnet werden. Xerohalophyten-Standorte weisen nach Breckle (1976) dann die höchsten Salzgehalte auf, wenn die Dürrebelastung vor Einsetzen stärkerer Regen besonders groß ist.

Laut Feldman et al. (2008) verursacht die Bodentrockenheit durch Salinität einen höheren Deckungsgrad von $\mathrm{C}_{4}$ - und CAM-Pflanzen in Regionen mit einem positiven bis neutralen Wasserhaushalt (Pampa und Espinal in Argentinien). Dieses konnte im Chaco, wo es sowieso ein gewisses Wasserdefizit gibt und $\mathrm{C}_{4}$ - und CAM-Pflanzen in allen Vegetationstypen ohne Rücksicht auf Bodensalinität dominieren, nicht nachgewiesen werden.

Viele Arten der Subfamilie Chenopodioideae (Amaranthaceae) sind für ihren Salzreichtum und ihre Natriumvorliebe bekannt. Natriumchlorid fördert bei vielen Chenopodioideen in bestimmten Konzentrationsbereichen das Wachstum (Albert 1982). Heterostachys ritteriana und Sarcocornia ambigua, zwei Arten der Chenopodioideae, kennzeichnen auch im Untersuchungsgebiet stark salzhaltige und natriumreiche Habitate.

Die Ausbildung mit Heterostachys ritteriana kennzeichnet sehr salzreiche und wechseltrockene Böden (Therburg 1997; Ragonese \& Piccinini (1977). Laut Rieger (1976) verändert sich die Heterostachys ritteriana-Gesellschaft nur unwesentlich in ihrer Physiognomie in den verschiedenen Jahreszeiten.

Grasfluren mit Spartina spartinae wachsen laut Feldman et al. (2007) in tiefergelegenen Zonen mit schwach dränierten, alkalischen und hydro-halomorphen Böden. Wie Studien aus 
Argentien zeigen, ist die Dynamik von Spartina spartinae-Grasfluren an häufige Brände angepasst (Feldman \& Lewis 2005; Feldman et al. 2009).

Die Cynodon niemfuensis-Gesellschaft zeigt einen starken Einfluss der Weidewirtschaft im Untersuchungsgebiet und kann somit als anthropogene Gesellschaft definiert werden. Die Leptochloa fusca subsp. uninervia- und die Sesuvium portulacastrum-Sporobolus pyramidatus-Gesellschaften sind Pioniere auf gestörten Standorten. Diese Störungen können sowohl natürlichen Ursprungs als auch anthropogen sein. An den Ufern der Lagunen und Flussbetten wachsen Sesuvium portulacastrum und Sporobolus pyramidatus häufig auf sandigen Sedimentaufschüttungen. Durch Sedimentaufschüttungen entstehen nach Ungar (1972) mikrotopographische Änderungen, die die Feuchtigkeit und Salinität reduzieren. Arten mit geringerer Salztoleranz besiedeln diese Aufschüttungen und so fängt eine Sukzessionreihe an. Bei den Sedimenten handelt es größtenteils um Sande und daher können laut Medina et al. (2008) Sesuvium portulacastrum und Sporobolus pyramidatus als Psammohalophyten bezeichnet werden.

Die weltweit verbreitete Ruppia maritima besitzt die wohl am weitesten angepasste Salztoleranz aller submersen Gefäßpflanzen. Die Keimung der Samen ist nach Untersuchungen in der Bucht von Florida jedoch bei geringen Salzgehalten am höchsten (La Peyre \& Rowe 2003). Im Chaco scheint diese Gesellschaft sehr stark von den Wetterbedingungen abhängig zu sein. Nach großen Niederschlägen und die damit verbundene Wasseransammlung und Reduzierung der Salinität werden die Keimung und das Wachstum dieser Pflanzen gefördert. 


\section{Gesellschaften und strukturelle Diversität der Gehölzvegetation salzhaltiger Standorte im paraguayischen Chaco}

\subsection{Einleitung}

Der Ost-West-Niederschlagsgradient im Chaco Boreal spielt auf Landschaftsebene eine entscheidende Rolle. Während der feuchte Ostchaco aus einem xeromesophytischen WaldPalmsavannen-Mosaik besteht, prägen xerophytische Waldformationen das Landschaftsbild im trockenen zentral-westlichen Chaco (Ramella \& Spichiger 1989, Spichiger et al. (1991), Prado 1993a). Die Struktur und Artenzusammensetzung der Waldgesellschaften werden laut Navarro et al. (2011) signifikant vom Niederschlagsgradient und von der Dränage-Kapazität des Bodens beeinflusst.

Die Wasserverfügbarkeit ist im Chaco ein offensichtlicher Minimumfaktor. Innerhalb der Vegetationszonen werden schon bei geringsten hydrischen Variationen Veränderungen der Pflanzengesellschaften und Lebensräume deutlich (Ramella \& Spichiger 1989, (Mitlöhner 1990). Geringe Niveauunterschiede von wenigen Zentimetern sowie Böden, die vom reinen Sand zum undurchlässigen Lösslehm oder Salzton reichen, schaffen Voraussetzungen für eine Fülle von verschiedenen Artenzusammensetzungen (Esser 1982).

Die Gehölzvegetation im paraguayischen Chaco ist in den letzten Jahrzehnten auf Landschafts- und Formationsebene in verschiedenen Studien untersucht worden (Tortorelli 1966, Esser 1982, Ramella \& Spichiger 1989, Huespe et al. 1991, Spichiger et al. 1991, Prado 1993a, 1993b, Mereles 2005). Von Spichiger et al. (1991) wird sowohl der xerophytische als auch der mesoxerophytische Landschaftskomplex im Chaco Boreal in jeweils zonale, extrazonale und azonale Formationen eingeteilt. In einer Übersicht der Vegetationsformationen im paraguayischen Chaco klassifiziert Mereles (2005) 22 Gehölzeinheiten und unterscheidet diese nach ihren hydrologischen und edaphischen Eigenschaften. Aus den unterschiedlichen Ansätzen geht hervor, dass die salzbeeinflussten Gebiete als eine Übergangszone angesehen werden können, die von zonalen Formationen sowohl des trockenen als auch des feuchten Chaco und azonalen Formationen geprägt wird.

Ökologische und pflanzensoziologische Untersuchungen der Gehölzvegetation im paraguayischen Chaco gibt es nur wenige. Entlang des Niederschlagsgradienten wurde von Mitlöhner (1990) die Konkurrenz der Holzgewächse in Trockenwald-Formationen und von Navarro et al. (2011) der Zusammenhang zwischen Bodeneigenschaften und Waldgesellschaften untersucht. Pflanzensoziologische Untersuchungen wurden von Galán de Mera (2001) sowohl im feuchten als auch im trockenen Chaco und von Navarro (2005) und Navarro et al. (2006) im Biosphärenreservat im Norden des paraguayischen Chaco durchgeführt. An den salzbeeinflussten Standorten gab es bisher noch keine ökologischpflanzensoziologischen Untersuchungen.

Anhand originaler pflanzensoziologischer Vegetationsaufnahmen und Strukturmessungen werden in diesem Kapitel die Gesellschaften der salzbeeinflussten Gehölzvegetation im paraguayischen Chaco beschrieben und sowohl ökologisch als auch strukturell charakterisiert. 


\subsection{Methoden}

\subsubsection{Vegetationsaufnahmen}

In den Sommermonaten 2010 - 2012 wurden in salzbeeinflusster Gehölzvegetation kontinuierliche und diskontinuierliche je ungefähr $100 \mathrm{~m}$ lange Transekte entlang des Salzgradienten (ausgehend von den Ufern der Salzstellen) angelegt. In jedem Untersuchungsort wurden jeweils ein kontinuierliches und mehrere diskontinuierliche Transekte untersucht. Insgesamt wurden 236 stratifiziert-randomisierte Probeflächen von 100 $\mathrm{m}^{2}$ in physiognomisch unterscheidbarer homogener Vegetation angelegt und pflanzensoziologisch untersucht (Anhang 5-1). In jeder Untersuchungsfläche wurden der Deckungsgrad sowie die maximale Höhe der Vegetation und jeder Vegetationsschicht geschätzt. Zudem wurden die Deckung der Streuschicht, des Totholzes und des offenen Bodens notiert. Von jeder Probefläche wurde nach Vegetationsschicht eine Artenliste angefertigt mit genauen Angaben zur Wuchsform und Artmächtigkeit jeder Art nach der erweiterten Braun-Blanquet-Skala (Braun-Blanquet 1964, Wilmanns 1998). Außerdem wurden alle Gehölze mit einem Brusthöhendurchmesser BHD $\geq 10 \mathrm{~cm}$ innerhalb der Untersuchungsflächen gezählt und ihre Höhe und BHD gemessen. Bei unter 1,3 m verzweigten Stämmen, wo mindestens ein Teilstamm einen BHD $\geq 10 \mathrm{~cm}$ erreichte, wurden alle Teilstämme einzeln gemessen und mit der Wurzel der Quadratsummen ein BHD-Wert für das jeweilige Individuum ermittelt.

Einzelne vom Boden her separate Stämme eines Individuums, wie es z.B. häufig bei Salta triflora zu beobachten ist, wurden als einzelne Individuen gemessen und notiert.

\subsubsection{Bodenanalysen}

In 61 Probeflächen wurde jeweils ein $40 \mathrm{~cm}$ tiefes Bodenprofil angelegt, um Bodenproben von den jeweiligen Bodenhorizonten zu entnehmen (Anhang 5-3).

Die Analysen der Bodenproben wurden im Departamento de Suelos y Ordenamiento Territorial der Fakultät für Agrarwissenschaften (Universidad Nacional de Asunción) durchgeführt. Von den Bodenproben wurden jeweils die Korngrößenverteilung, pH-Wert, Organische Substanz, Calcium-, Magnesium- und Natriumgehalt sowie die Elektrische Leitfähigkeit analysiert.

Die Bestimmung der Korngrößenverteilung erfolgte durch das Aräometer-Verfahren nach Bouyoucos (1962). Der Gehalt an organischer Substanz wurde durch die Walkley-BlackMethode (Lichterfelder Methode) mit nasser Veraschung der organischen Substanz mittels eines Gemisches aus Kaliumdichromat und Schwefelsäure ermittelt (nach Soil Survey Staff 1996).

Mittels eines $\mathrm{pH}-$ Meters wurde die Bodenreaktion (pH-Wert) potentiometrisch in destilliertem Wasser gemessen. Die elektrische Leitfähigkeit (Salinität) wurde in konstanter Temperatur von $25^{\circ} \mathrm{C}$ konduktometrisch mit einem Leitfähigkeitsmessgerät ermittelt. Die Bestimmung der Anteile wasserlöslicher Kationen (Calcium, Magnesium und Natrium) erfolgte mittels Atomabsorptionsspektrometrie. 
Für die statistische Auswertung wurden die analysierten Bodenparameter der Horizonte eines jeden Profils proportional in eine jeweils $20 \mathrm{~cm}$ mächtige obere und untere Bodenschicht umgerechnet.

\subsubsection{Datenauswertung}

\section{Klassifikation und multivariate Analysen}

Die im Gelände aufgenommenen Artdaten wurden in die Vegetationsdatenbank Turboveg (Hennekens \& Schaminée 2001) eingetragen. Die Klassifikation der Vegetationseinheiten wurde mit der in JUICE (Tichý 2002) enthaltenen und nach Roleček et al. (2009) modifizierten TWINSPAN-Analyse (two-way indicator species analysis; Hill 1979) durchgeführt. Damit die TWINSPAN-Analyse eine Gewichtung von sehr abundanten Arten gegenüber den weniger abundanten vornehmen konnte, wurden Pseudospecies und Cut Levels gesetzt. Die 4 Cut Levels wurden auf 0, 5, 25 und 50 \% Deckungsgrad festgelegt.

Mit dem Programm CANOCO, Version 4.5 (ter Braak \& Šmilauer 2002) wurde mittels Ordinationen die Klassifikation der Vegetationseinheiten überprüft und eventuelle Zusammenhänge zwischen der Artenzusammensetzung bzw. den Vegetationsaufnahmen und den standörtlichen Parametern untersucht.

Um herauszufinden, wie hoch die Variabilität und Heterogenität in der Artenzusammensetzung der Vegetationsaufnahmen ist, wurden Korrespondenzanalysen (DCA - Detrended Correspondence Analysis, Hill \& Gauch 1980) durchgeführt. Dabei wurden seltene Arten abgewichtet und eine log-Transformierung vorgenommen. Bei Datensätzen mit einer Gradientenlänge $\leq 3$ wurde eine Hauptkomponentenanalyse (PCA principal component analysis) verwendet.

Um bei der direkten Gradientenanalyse (CCA - Canonical Correspondence Analysis, ter Braak 1987) herauszufinden, wie signifikant die Bodenparameter mit der Artenzusammensetzung in den Aufnahmen in Verbindung standen, wurde ein unbeschränkter Monte-Carlo-Permutationstest mit 999 Permutationen durchgeführt. Die Parameter wurden schrittweise reduziert und auf ihren Zusammenhang mit der Artenverteilung getestet. Wie Leyer \& Wesche (2007) vorschlagen, wurden dabei nur diejenigen in das Regressionsmodell einbezogen, die einen signifikanten Beitrag $(\mathrm{p} \leq 0,05)$ zur Erklärung der Artenverteilung lieferten.

\section{Strukturanalysen}

Anhand von Höhen- und Brusthöhendurchmesser (BHD)-Messungen aller Gehölze mit einem BHD $\geq 10 \mathrm{~cm}$ in den jeweiligen Probeflächen wurde die Struktur der Arten und Vegetationseinheiten analysiert.

In Anlehnung an Lamprecht (1986) wurden die absolute Abundanz (Individuen/Art), absolute Frequenz (Auftreten bzw. Fehlen einer Art in den einzelnen Probeflächen) und absolute Dominanz (Summe der individuellen Stammkreisflächen in $\mathrm{m}^{2}$ einer Art) für jede Art pro Vegetationseinheit ermittelt. 
Um annähernde strukturelle Vergleiche der betreffenden Vegetationseinheiten zu ermöglichen, wurde der Bedeutungswert-Index (IVI - Importance-Value-Index) nach Curtis \& McIntosh (1951) für jede Art berechnet. Der IVI besteht aus der Summe der relativen Abundanz (porzentualer Anteil jeder Art an der Gesamt-Individuenzahl), relativen Frequenz (prozentualer Anteil an der Summe der absoluten Frequenzen) und relativen Dominanz (prozentualer Anteil einer Art an der Gesamtstammkreisfläche).

Die univariat-statistischen Analysen wurden mit den Programmen Excel und $R$ durchgeführt.

\subsection{Ergebnisse}

\subsubsection{Klassifikation und Beschreibung der Gesellschaften}

In den salzbeeinflussten Habitaten wurden anhand originaler Vegetationsaufnahmen zehn Vegetationeinheiten unterschieden. Die differenzierten Gesellschaften kommen entlang eines Gradienten unterschiedlicher Wasserverfügbarkeit und Salinität vor. Die Arten- und Strukturdiversität der Gehölzvegetation nimmt von den salzreichen zu den salzarmen Standorten zu und der Anteil an halophilen Arten ab (Tabelle 5-1 und Anhang 5-2).

Die Gehölzgesellschaften auf salzreichen und periodisch überfluteten Böden werden in DCAOrdinationsdiagramm (Abb. 5-2) dargestellt. Die erste Achse zeigt den Feuchtigkeitsgradienten von der hygrohalophilen Parkinsonia aculeata-Copernicia alba-Gesellschaft bis zur xerohalophilen Piptadeniopsis lomentifera-Lycium americanum-Gesellschaft. Die Verteilung der Gesellschaften auf der zweiten Achse wird vom Salzgehalt im Oberboden bestimmt.

10) Parkinsonia aculeata-Copernicia alba-Gesellschaft: offene $4 \mathrm{~m}$ hohe Gebüschgesellschaft, in der Parkinsonia aculeata dominiert. Die neotropisch weitverbreitete Pionierstrauchart Parkinsonia aculeata besiedelt im paraguayischen Chaco meist tonige, saure und staunasse Standorte in periodisch überfluteten Senken. Im feuchten Chaco kommt diese Art oft in gestörten Copernicia alba-Palmsavannen vor.

Im Untersuchungsgebiet wurde diese Gesellschaft in feuchten Einschwemmsenken oder an den Ufern der Lagunen gefunden (Abb. 5-1). Die Strauchschicht besteht aus Parkinsonia aculeata und einzelnen Copernicia alba-Palmen. Die Differentialarten der Krautschicht sind Parkinsonia aculeata, Copernicia alba, Euphorbia serpens und Feuchtezeiger wie Neptunia pubesens, Solanum glaucophyllum,

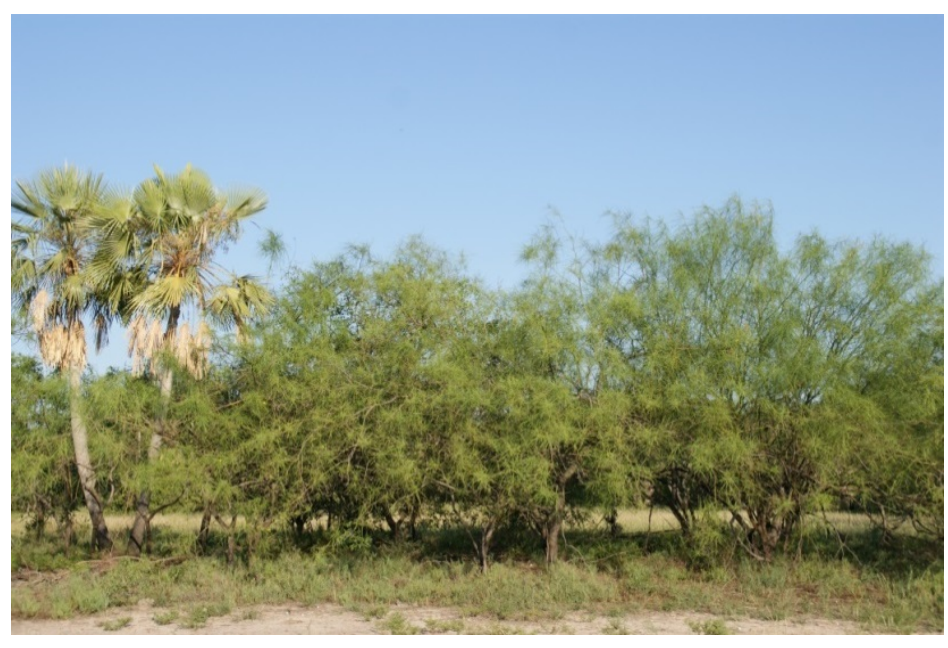
Alternanthera paronychioides ssp. Abb. 5-1: Parkinsonia aculeata-Copernicia alba-Gesellschaft chacoensis, Setaria geminata und an der Laguna Rojas Silva.

Sesbania virgata. 


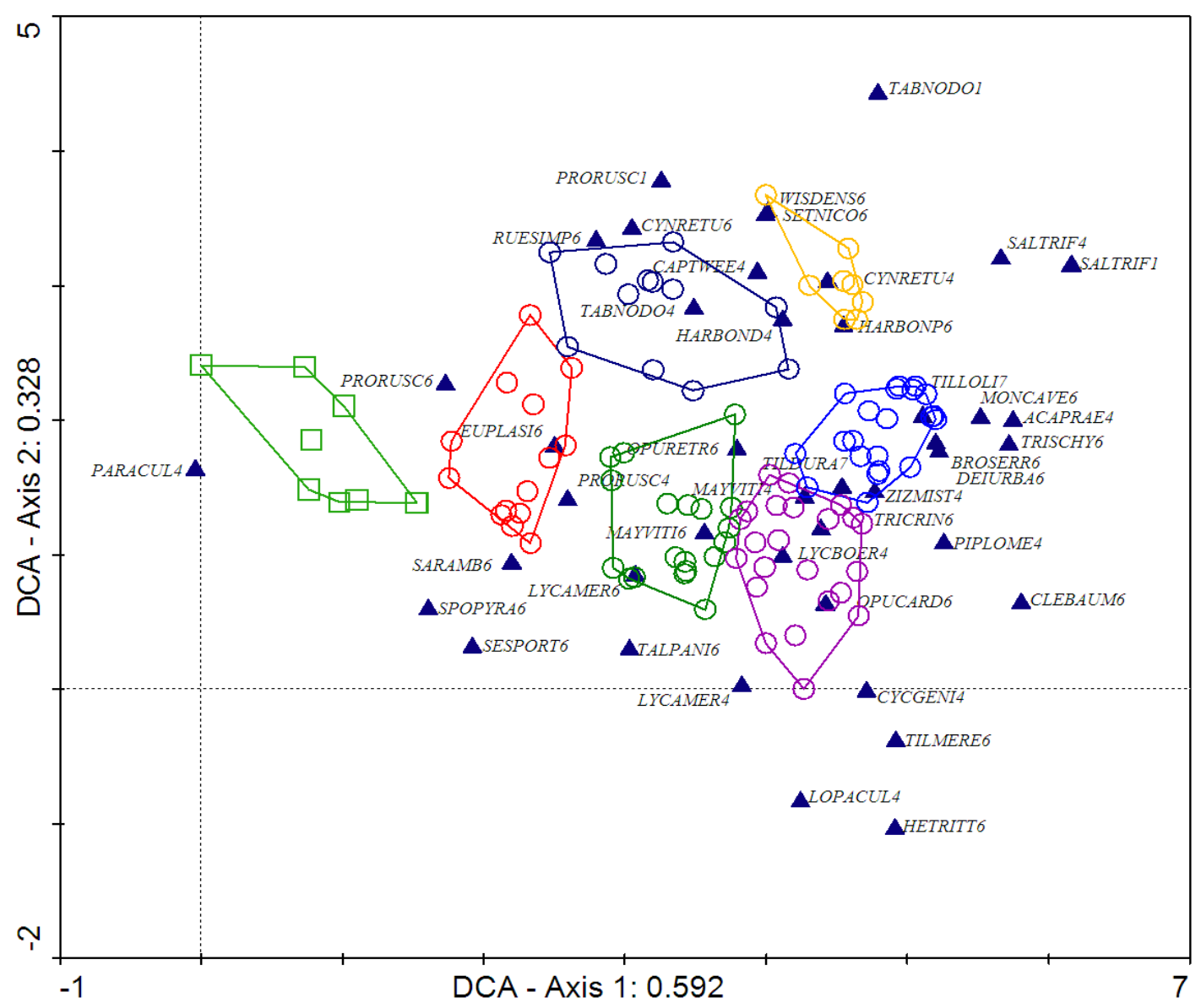

Abb. 5-2: DCA - Ordinationsdiagramm der Gehölzgesellschaften auf salzhaltigen und periodisch überfluteten Böden. Abgebildet werden 43 Arten und die Vegetationsaufnahmen (insgesamt 107) der jeweiligen Pflanzengesellschaften: $\square$ 10) Parkinsonia aculeata-Copernicia alba-Gesellschaft, $\bigcirc$ 11) Prosopis ruscifolia-Sporobolus pyramidatus-Gesellschaft, $\bigcirc$ 12) Prosopis ruscifola-Evolvulus convolvuloides-Gesellschaft, $\bigcirc$ 13) Prosopis ruscifolia-Lycium americanum-Gesellschaft, $\bigcirc$ 14) Lycium americanum-Cyclolepis genistoides-Gesellschaft, $\bigcirc$ 15) Prosopis ruscifolia-Tabebuia nodosa-Gesellschaft, 16) Piptadeniopsis lomentifera-Lycium americanum-Gesellschaft, $\mathbf{\Delta}$ zeigt die Position der jeweiligen Arten (Artnamen zu den jeweiligen Abkürzungen im Anhang 5-2, Log-file-Daten im Anhang 5-4).

Begleitend treten im Untersuchungsgebiet krautige Halophyten wie Heliotropium curassavicum, Sporobolus pyramidatus, Sesuvium portulacastrum, Sarcocornia ambigua und Prosopis ruscifolia auf.

11) Prosopis ruscifolia-Sporobolus pyramidatus-Gesellschaft: Pioniergebüsch auf tonigen, salzhaltigen und periodisch überfluteten Böden. Die offene und $4 \mathrm{~m}$ hohe Strauchschicht wird von Prosopis ruscifolia dominiert (Abb. 5-3). Weitere Differentialarten sind Copernicia alba und Lycium nodosum.

Die Krautschicht besteht aus Euphorbia lasiocarpa, Stemodia ericifolia und halophilen Arten wie Sporobolus pyramidatus, Sesuvium portulacastrum, Sarcocornia ambigua, Heliotropium curassavicum und Atriplex eximia. 
An den Ästen des Prosopis ruscifolia ist häufig die hemiparasitischepiphytische Phoradendron argentinum (Santalaceae) zu beobachten.

Obwohl diese Gesellschaft räumlich direkt neben der Parkinsonia aculeata-Copernicia alba-Gesellschaft vorkommt, kann sie durch das Auftreten von Atriplex eximia und das Fehlen von Feuchtezeigern wie Parkinsonia aculeata, Neptunia pubescens und Sesbania virgata gut unterschieden werden.

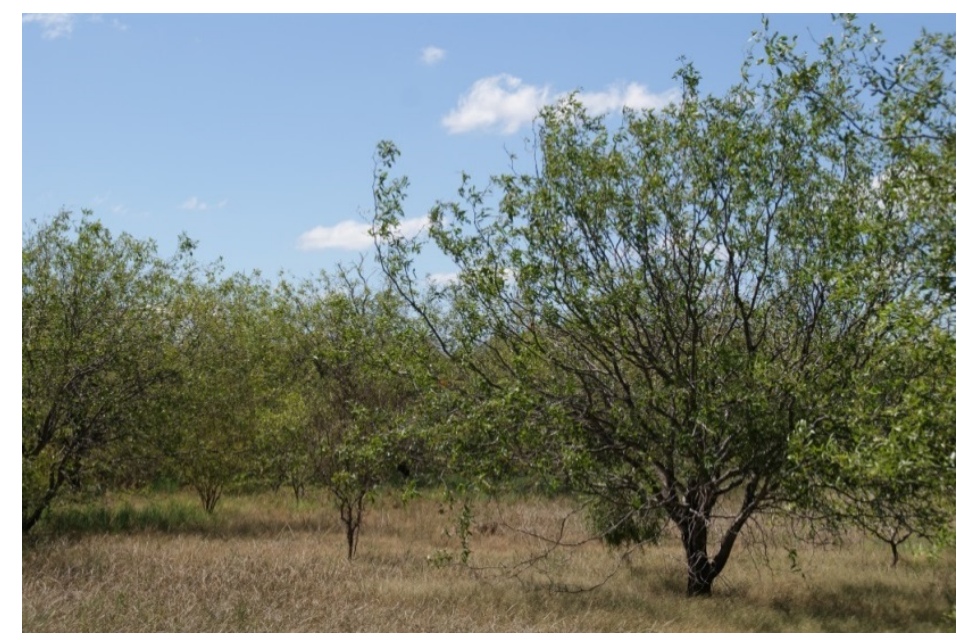

Abb. 5-3: Prosopis ruscifolia-Sporobolus pyramidatus-Gesellschaft am Riacho González.

12) Prosopis ruscifolia-Evolvulus convolvuloides-Gesellschaft: Diese hygrophile Einheit (Abb. 4-4) kommt in periodisch überfluteten Senken mit tonigen, schwach dränierten Böden vor. Die dominante Art in der Strauch- und Baumschicht Prosopis ruscifolia kann in einigen Fällen eine Höhe von 6 m erreichen. Die Gebüschgesellschaft erreicht eine mittlere Höhe von 3,5 m. Weitere Differentialarten in der offenen Strauchschicht sind Acacia caven und Capparicordis tweediana. Begleitende Arten sind Cynophalla retusa und Tabebuia nodosa.

Differentialarten der Krautschicht sind Evolvulus convolvuloides, Desmanthus virgatus, Lycium nodosum, Prosopis hassleri, Ayenia odonellii, Ruellia simplex und Setaria nicorae. Das Auftreten von Evolvulus convolvuloides und Ayenia odonellii und das Fehlen krautiger und zwergstrauchartiger Halophyten, wie Sporobolus pyramidatus, Sesuvium portulacastrum, Sarcocornia ambigua und Lycium americanum unterscheidet diese Gesellschaft von anderen Prosopis ruscifolia-Gesellschaften.

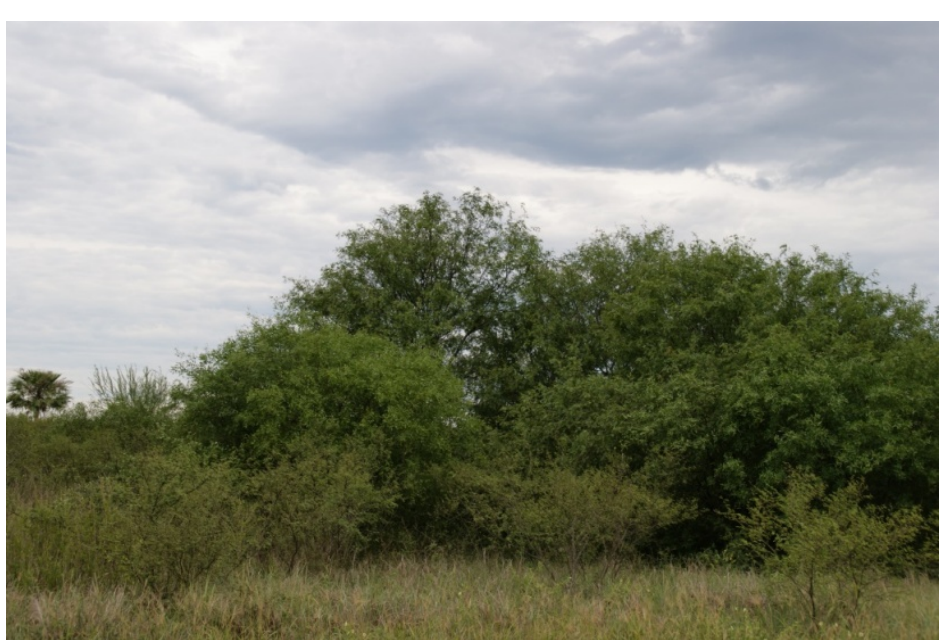

Abb. 5-4: Prosopis ruscifolia-Evolvulus convolvuloides-Gesellschaft in der Reserva Natural Yaguarete Porã.

13) Prosopis ruscifolia-Lycium americanum-Gesellschaft: Gebüschgesellschaft mit zwei klar ausgebildeten Strauchschichten. Die untere Strauchschicht, die von Lycium americanum dominiert wird, erreicht eine durchschnittliche Höhe von 1,2 m. Die obere Strauchschicht erreicht $4 \mathrm{~m}$ und wird von Prosopis ruscifolia dominiert. Eine Baumschicht fehlt oder es kommen nur einzelne niedrige Bäume vor. Weitere Differentialarten der Strauchschicht sind Lophocarpinia aculeatifolia, Prosopis rojasiana und Maytenus vitis-idaea. 
Typische Arten in der Krautschicht sind Sesuvium portulacastrum, Sporobolus pyramidatus, Sarcocornia ambigua, Talinum paniculatum und Sclerophylax spinescens.

Im Gegensatz zur Prosopis ruscifolia-Sporobolus pyramidatus-Gesellschaft fehlt in dieser Gesellschaft Copernicia alba und es treten xerohalophile Gehölzarten auf. In der Krautschicht werden Feuchtezeiger durch sukkulente Krautarten ersetzt und Heliotropium curassavicum kommt nicht mehr vor.

14) Lycium americanum-Cyclolepis genistoides-Gesellschaft: diese Gebüschgesellschaft kommt in meist nur 10 m breiten Streifen an den Rändern trockener Salzpfannen vor (Abb.55). Sie ist ebenfalls durch zwei Strauchschichten gekennzeichnet. Die Differentialarten in der unteren Strauchschicht, die eine durchschnittliche Höhe von 1,6 m erreicht, sind Cyclolepis genistoides und Lycium americanum. Die obere Strauchschicht erreicht eine Höhe von $4 \mathrm{~m}$ und besteht aus Prosopis ruscifolia, Lophocarpinia aculeatifolia und Lycium boerhaviaefolium. Als begleitende Art tritt häufig Maytenus vitis-idaea auf.

Die Kennart der Krautschicht ist die auf dem Boden lebende Tillandsia mereliana. Weitere Differentialarten der Krautschicht sind Deinacanthon urbanianum, Opuntia elata var. cardiosperma, Talinum paniculatum, Trichloris crinita und Heterostachys ritteriana, die meist am äußeren Rand zu den

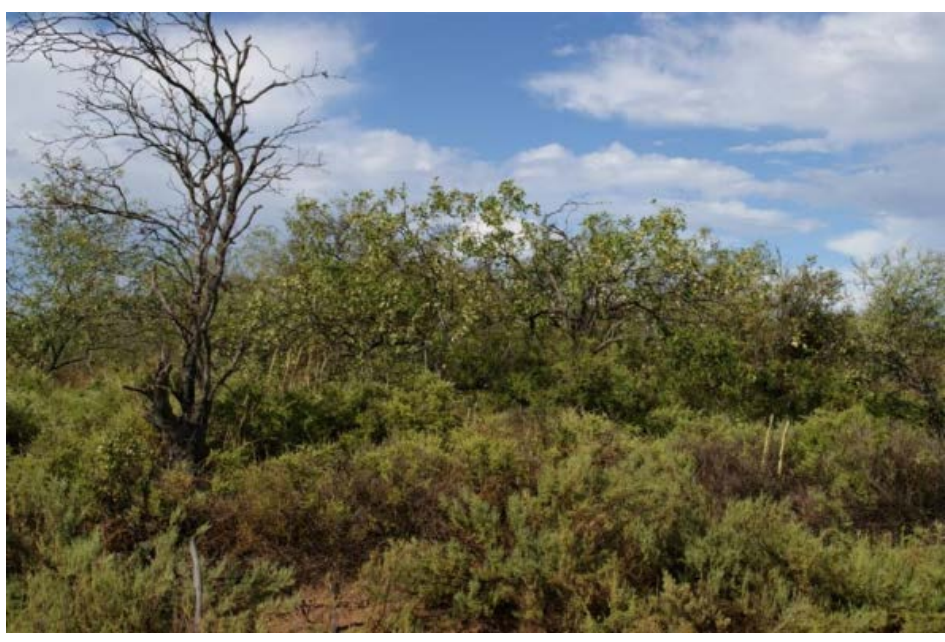

Abb. 5-5: Lycium americanum-Cyclolepis genistoides-Gesellschaft am Rand der Salzpfannen im Naturreservat Chaco Lodge Salzpfannen vorkommt. Typische Epiphyten dieser Gesellschaft sind Tillandsia mereliana und Tillandsia duratii var. saxatilis.

Diese Gesellschaft kann von der Prosopis ruscifolia-Lycium americanum-Gesellschaft durch das Auftreten von Cyclolepis genistoides, Tillandsia mereliana, Heterostachys ritteriana und Deinacanthon urbanianum unterschieden werden. Halophyten wie Sesuvium portulacastrum, Sporobolus pyramidatus und Sarcocornia ambigua kommen nur noch vereinzelt vor.

15) Prosopis ruscifolia-Tabebuia nodosa-Gesellschaft (15): hygro-phile Waldgesellschaft, die eine mittlere Höhe von 6,4 m erreicht und in periodisch überfluteten Senken vorkommt (Abb. 5-6).

Differentialarten der Baumschicht sind Prosopis ruscifolia, Tabebuia nodosa, Caesalpinia paraguariensis und Salta triflora. In der Strauchschicht, die eine mittlere Höhe von 3,7 m erreicht, kommen Salta triflora, Achatocarpus praecox, Cynophalla retusa, Maytenus vitisidaea und Bougainvillea campanulata vor. 


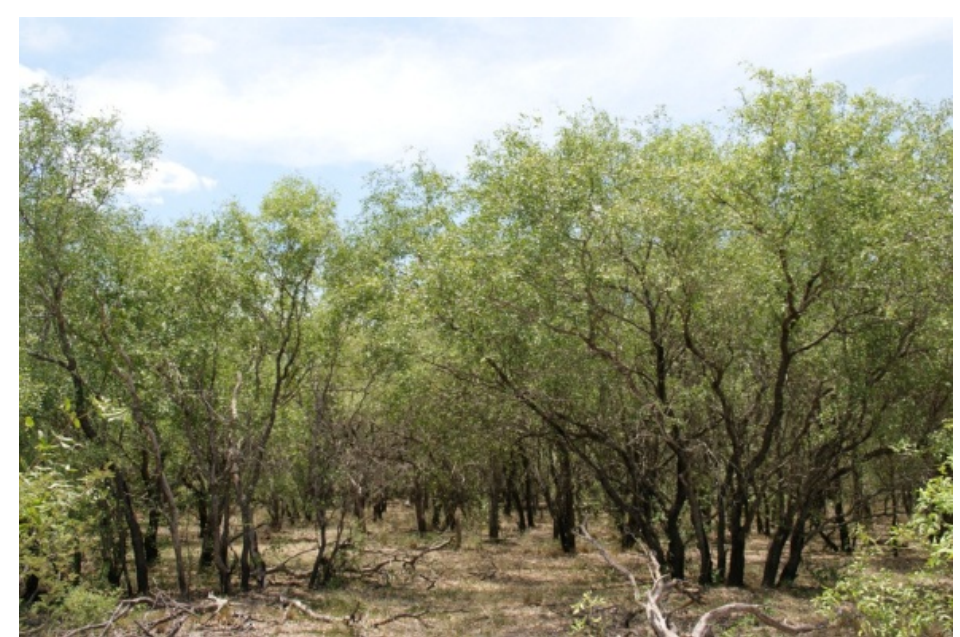

Abb. 5-6: Prosopis ruscifolia-Tabebuia nodosa-Gesellschaft in der Reserva Natural Fortín Salazar.
Differentialarten der Krautschicht sind Ruellia simplex, Ruellia hygrophila, Aechmea distichanta var. schlumbergeri und Carlowrightia sulcata. Begleitend treten Harrisia bonplandii und Setaria nicorae auf.

Charakteristische Epiphyten sind Selenicereus setaceus und Tillandsia recurvifolia.

16) Piptadeniopsis lomentifera-Lycium americanum-Gesellschaft: Zwischen den halophilen Gebüschgesellschaften und den Waldgesellschaften kann häufig eine heterogene Übergangsgesellschaft, in der Arten mit höherer Stetigkeit fehlen, beobachtet werden. Bei der Einheit handelt es sich um eine niedrige Waldgesellschaft (mittlere Höhe von 5 m), die in der Baumschicht aus Piptadeniopsis lomentifera, Bougainvillea campanulata, Salta triflora und Ziziphus mistol besteht. Selten treten Ceiba chodatii und Stetsonia coryne auf.

Differentialarten der Strauchschicht sind Piptadeniopsis lomentifera, Lycium americanum, Prosopis rojasiana, Lycium boerhaaviaefolium, Trithrinax schizophylla und Cereus forbesii. Begleitende und weitverbreitete Arten sind Maytenus vitis-idaea, Cynophalla retusa, Sarcotoxicum salicifolium, Salta triflora und Acacia praecox.

Differentialarten der Krautschicht sind Lycium americanum, Alternanthera kurtzii, Echinopsis rhodotricha, Monvillea cavendishii, Cleistocactus baumannii, Trichloris crinita, Deinacanthon urbanianum, Bromelia hieronymi, Setaria nicorae und Wissadula densiflora. Selten treten Selaginella sellowii, Sida dictyocarpa, Opuntia elata var. cardiosperma und Basistemon spinosus auf.

Charakteristische Lianen und Epiphyten sind Marsdenia castillionii, Tillandsia reichenbachii und die weitverbreiteten Tillandsia loliacea und Tillandsia duratii var. saxatilis.

Diese Gesellschaft kann von der Lycium americanum-Cyclolepis genistoides Gesellschaft durch die differenzierte Baumschicht mit Piptadeniopsis lomentifera, Ziziphus mistol und Salta triflora sowie durch das Fehlen krautiger Halophyten und das seltene Auftreten von Prosopis ruscifolia, Cyclolepis genistoides und Tillandsia mereliana unterschieden werden. Im Gegensatz zur Bulnesia sarmientoi-Gouinia paraguayensis Gesellschaft treten Trichloris crinita und Tillandsia reichenbachii auf. Außerdem kommen Bulnesia sarmientoi, Ruellia simplex und Gouinia paraguayensis selten oder gar nicht vor. 
Die Waldgesellschaften treten meist, wie im Vegetationsprofil (Abb. 5-9) ersichtlich ist, an etwas höher gelegenen Standorten im Anschluss an die oben beschriebenen halophilen Gebüschgesellschaften auf. Die zwei Waldgesellschaften (Einheiten 17 und 18) auf schwach bis mäßig dränierten Böden werden in einem PCA-Ordinationsdiagramm (Abb. 5-7) dargestellt. Die Bodenart und die damit zusammenhängende Dränung des Bodens bestimmt die Verteilung der Arten auf der ersten Achse.

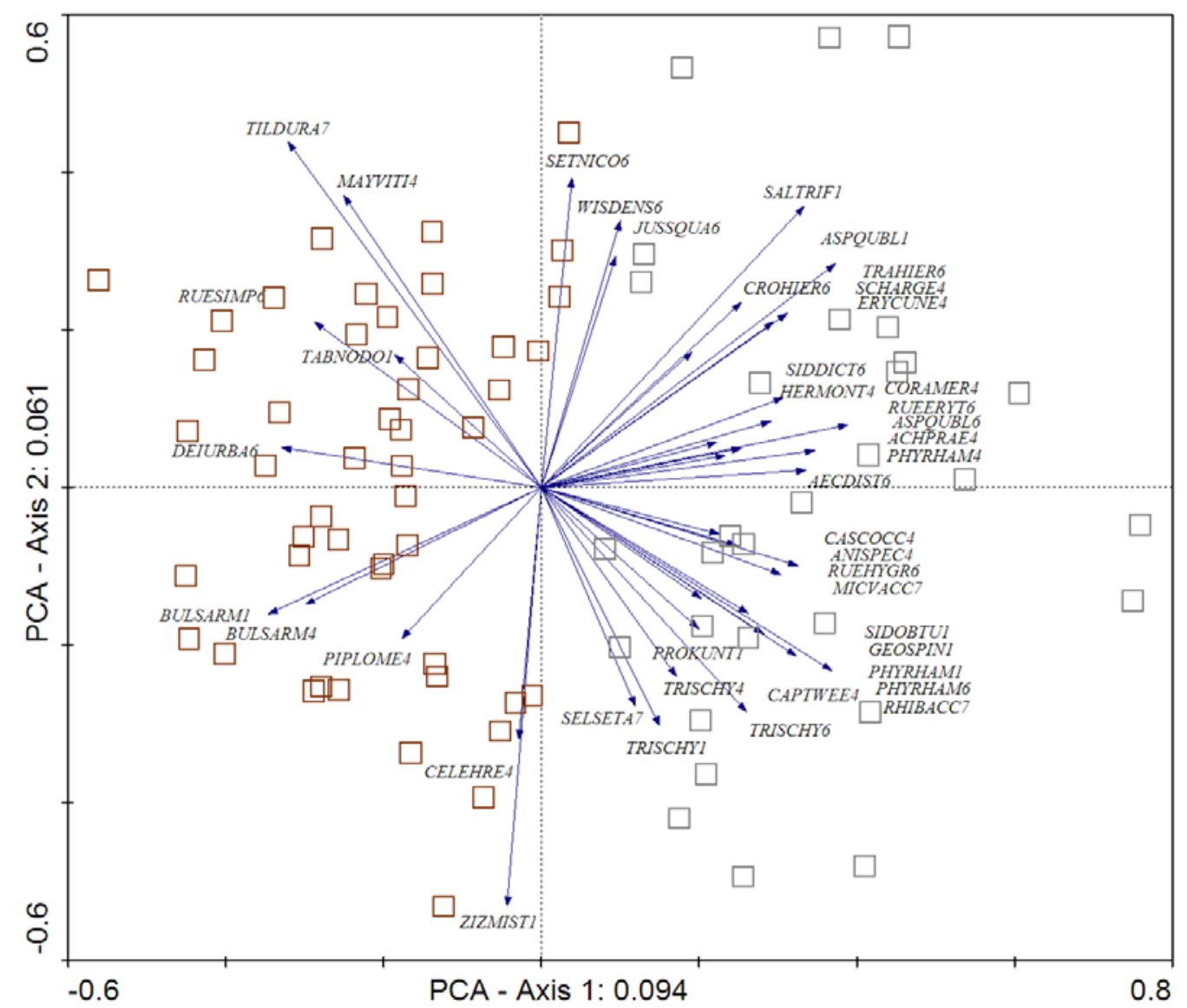

Abb. 5-7: PCA - Ordinationsdiagramm der Waldgesellschaften auf schwach bis mäßig dränierten Böden. Abgebildet werden 42 Arten und die Vegetationsaufnahmen (insgesamt 77) der jeweiligen Pflanzengesellschaften: $\square$ 17) Bulnesia sarmientoi-Gouinia paraguayensis-Gesellschaft, $\square$ 18) Phyllostylon rhamnoides-Trithrinax schizophylla-Gesellschaft, $\rightarrow$ zeigt die Position der jeweiligen Arten (Artnamen zu den jeweiligen Abkürzungen im Anhang 5-2, Log-file-Daten im Anhang 5-5)

17) Bulnesia sarmientoi-Gouinia paraguayensis-Gesellschaft: ist eine Waldgesellschaft auf schwach dränierten und salzhaltigen Böden (Abb. 5-8). Differentialarten der Baumschicht, die eine mittlere Höhe von 6,9 m erreicht, sind Bulnesia sarmientoi, Tabebuia nodosa, Ziziphus mistol, Salta triflora und Sideroxylon obtusifolium. Selten auftretende Arten sind Prosopis sericantha und Ceiba chodatii.

Die Strauchschicht, die eine mittlere Höhe von 3,4 m erreicht, besteht aus Celtis ehrenbergiana, Bougainvillea campanulata, Sarcotoxicum salicifolium, Sideroxylon obtusifolium und Trithrinax schizophylla. Begleitende und weitverbreitete Arten sind Maytenus vitis-idaea, Salta triflora, Acacia praecox und Cynophalla retusa. 
Differentialarten der Krautschicht sind Gouinia paraguayensis, Bromelia serra, Bromelia hieronymi, Justicia xylosteoides, Monvillea spegazzinii, Monvillea cavendishii und Alternanthera kurzii. Begleitarten sind Deinacanthon urbanianum, Salta triflora, Setaria nicorae.

Eine häufig anzutreffende Epiphytenart dieser Gesellschaft ist Tillandsia duratii var. saxatilis.

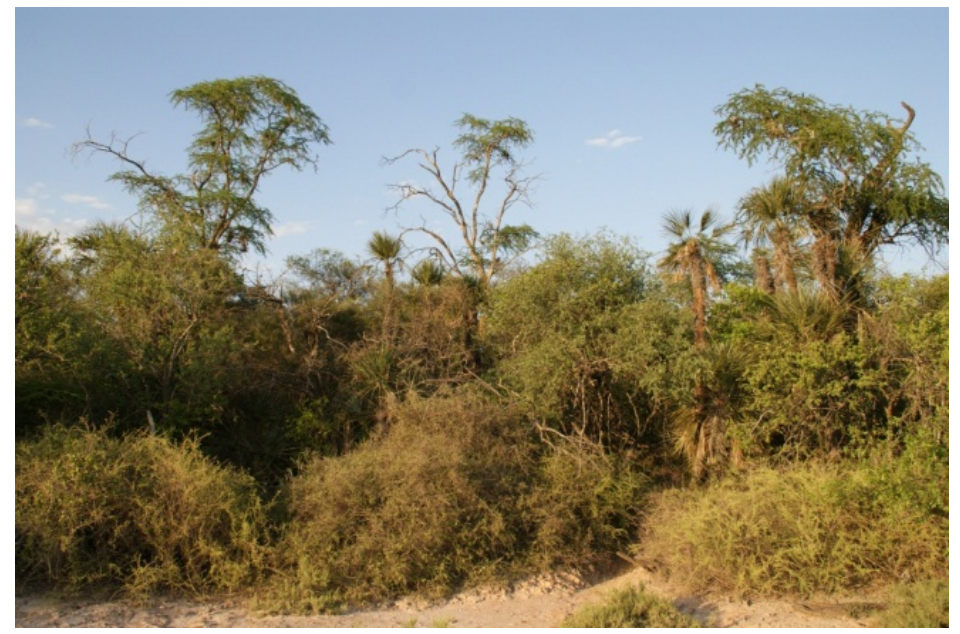

Abb. 5-8: Die Bulnesia sarmientoi-Gouinia paraguayensisGesellschaft ist im gesamten Untersuchungsgebiet sehr häufig anzutreffen.

18) Phyllostylon rhamnoides-Trithrinax schizophylla-Gesellschaft: Waldgesellschaft an mäßig dränierten Standorten. Die Baumschicht erreicht eine mittlere Höhe von $8 \mathrm{~m}$ und besteht in den meisten Fällen aus einer oberen und einer unteren Schicht. Differentialarten der oberen Baumschicht sind Aspidosperma quebracho-blanco, Bulnesia sarmientoi und Ceiba chodatii. Die untere Baumschicht besteht aus Phyllostylon rhamnoides, Ximenia americana, Sideroxylon obtusifolium und Trithrinax schizophylla. Begleitarten sind Ziziphus mistol und Salta triflora.

Die Strauchschicht besteht aus Trithrinax schizophylla, Achatocarpus praecox, Schaefferia argentinensis, Anisocapparis speciosa, Celtis ehrenbergiana, Phyllostylon rhamnoides und Pisonia hassleriana. Begleitarten sind Cynophalla retusa, Capparicordis tweediana und Acacia praecox.

Differentialarten der Krautschicht sind Rivina humilis, Sida dictyocarpa, Croton hieronymi, Tragia hieronymi, Ruellia erythropus, Ruellia hygrophila, Ditaxis montevidensis, Aechmea distichantha var. schlumbergeri und Bromelia serra.

Typische Lianen und Epiphyten dieser Waldgesellschaft sind Fridericia dichotoma, Herreria montevidensis, Ipomoea amnicola, Microgramma vaccinifolia und die häufig anzutreffende Tillandsia loliacea.

Trennarten zur Bulnesia sarmientoi-Gouinia paraguayensis-Gesellschaft sind Phyllostylon rhamnoides, Schaefferia argentinensis, Achatocarpus praecox, Ruellia erythropus, Tragia hieronymi und Herreria montevidensis.

19) Schinopsis cornuta-Elionurus muticus-Gesellschaft: Im engen Kontakt mit der Gehölzvegetation in salzbeeinflussten Habitaten tritt am Riacho Yakaré Sur eine Baumsavannen-Gesellschaft auf, die im paraguayischen Chaco fossile mit Sand gefüllte Flusssysteme besiedelt. Im Untersuchungsgebiet kommt diese Einheit an höher gelegenen Standorten in einer Entfernung von 200 bis 500 m von den Salzniederungen vor (Abb. 5-9 und 5-11). 
Umringt von salzbeeinflusster Vegetation, konnte bei dieser Gesellschaft kein direkter Kontakt zum lokalen salzhaltigen Grundwasser nachgewiesen werden, da die artenreiche Krautschicht größtenteils aus Glykophyten besteht.

Einzelne Bäume, die eine mittlere Höhe von $7 \mathrm{~m}$ erreichen, sind Schinopsis cornuta, Tabebuia aurea und Astronium fraxinifolium Die Strauchschicht fehlt in den meisten

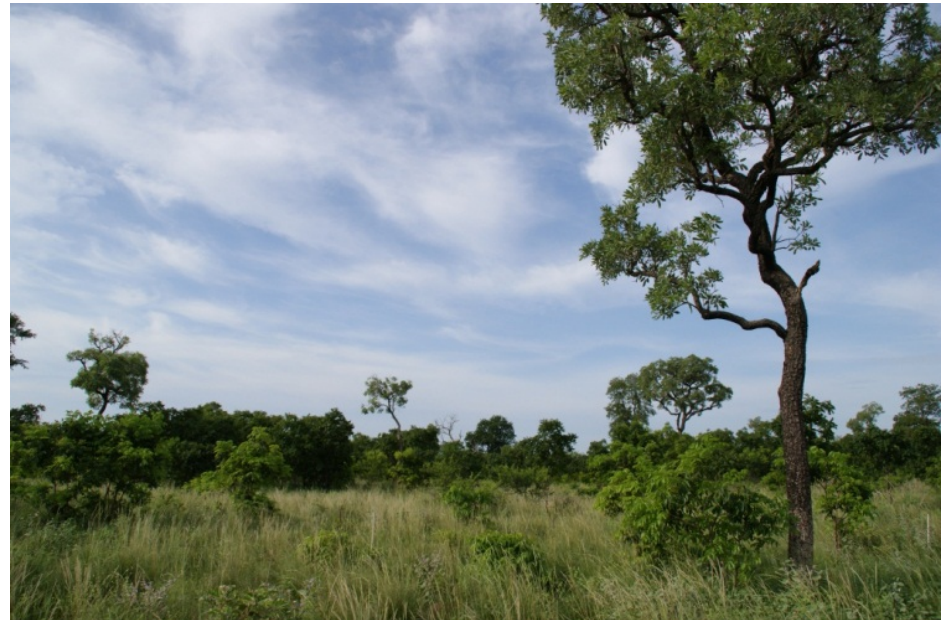

Abb. 5-9: Schinopsis cornuta-Elionurus muticus-Gesellschaft im Ramsargebiet Laguna Rojas Silva.

Fällen. Pioniersträucher wie Acacia

aroma und Prosopis hassleri sind Störungszeiger in dieser Gesellschaft.

Die artenreiche Krautschicht wird von Aristida mendocina, Elionurus muticus, Pappophorum krapovickasii, Rhynchospora burkartii, Urochloa paucispicata, Melochia werdermanni und Astraea lobata dominiert. Weitere Differentialarten sind Cleome tucumanensis, Cnidosculus albomaculatus, Jacquemontia decumbens, Microstachys hispida, Malvastrum coromandelianum, Porophyllum ruderale, Vernonia niederleinii, Zornia latifolia, Evolvulus sericeus, unter anderen.

Im Westen des Untersuchungsgebietes wurde eine Verarmung der Krautschicht, eine starke Verbuschung und eine außerordentliche Dominanz von Schinopsis cornuta beobachtet, was womöglich auf den starken anthropogenen Einfluss und den daraus resultierenden Versalzungseffekt zurückzuführen sein dürfte (Abb. 5-10).

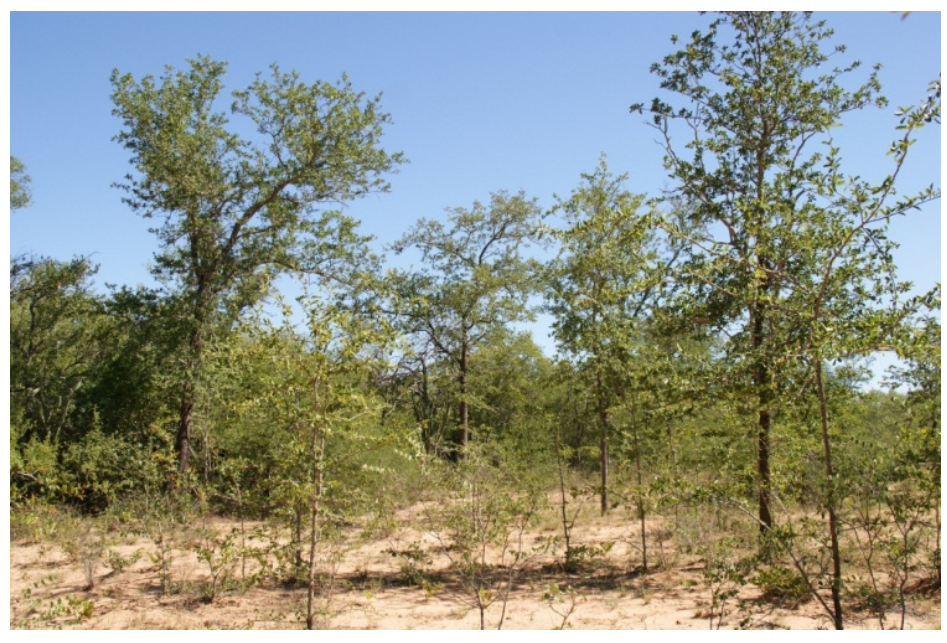

Abb. 5-10: Stark verarmte Schinopsis cornuta-Elionurus muticusGesellschaft in der Umgebung von Laguna Ganso. 


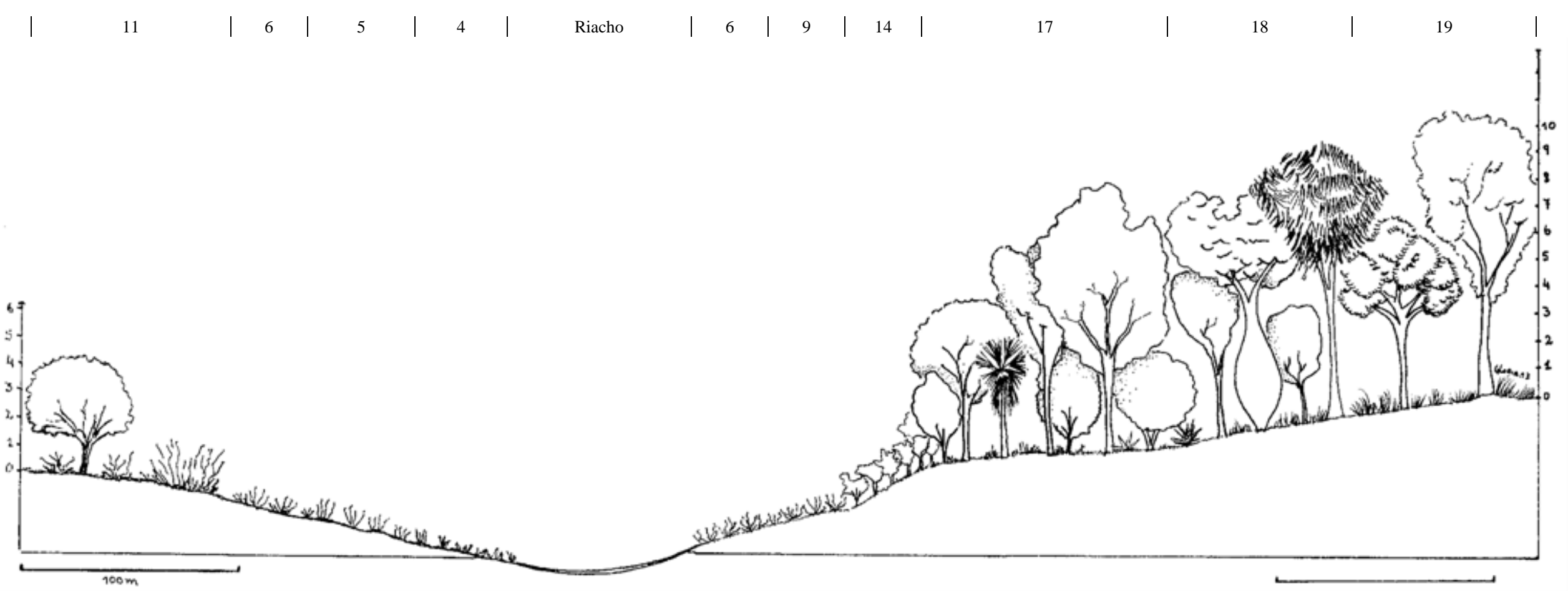

Abb. 5-11: Vegetationsprofil am Riacho Yakaré Sur. Abgebildet werden sowohl Salzmarsch- als auch Gehölzgesellschaften: 4) Cynodon niemfuensis-Gesellschaft, 5) Leptochloa fusca subsp. uninervia-Gesellschaft, 6) Sarcocornia ambigua-Gesellschaft, 9) Heterostachys ritteriana-Gesellschaft, 11) Prosopis ruscifolia-Sporobolus pryramidatus-Gesellschaft, 14) Lycium americanum-Cyclolepis genistoides-Gesellschaft, 17) Bulnesia sarmientoi-Gouinia paraguayensis-Gesellschaft, 18) Phyllostylon rhamnoides-Trithrinax schyzophylla-Gesellschaft und 19) Schinopsis cornuta-Elionurus muticus-Gesellschaft. 
Tab. 5-1: Synoptische Tabelle der Gehölzvegetation mit Stetigkeitsangaben in \%. Nur Arten mit Stetigkeiten $>20 \%$ in wenigstens einer Gesellschaft sind enthalten. Die Deckungsgrade und die Höhe der Vegetationsschichten werden jeweils mit dem Mittelwert und der Standardabweichung angegeben.

\begin{tabular}{|c|c|c|c|c|c|c|c|c|c|c|}
\hline Vegetationseinheit & 10 & 11 & 12 & 13 & 14 & 15 & 16 & 17 & 18 & 19 \\
\hline Anzahl Aufnahmen & 9 & 14 & 12 & 19 & 23 & 14 & 31 & 63 & 35 & 16 \\
\hline Mittl. Artenanzahl/Aufnahmefläche & 12.7 & 14.2 & 20.2 & 14.9 & 21.4 & 23.3 & 31.2 & 28.6 & 37.3 & 23.7 \\
\hline Artenanzahl & 44 & 75 & 103 & 79 & 99 & 74 & 154 & 177 & 196 & 122 \\
\hline \multirow[t]{2}{*}{ Gesamtdeckung (\%) } & 68.9 & 75.7 & 79.8 & 85.2 & 92.7 & 81.9 & 90.0 & 88.0 & 92.2 & 76.4 \\
\hline & \pm 13.4 & \pm 11.9 & \pm 10.3 & \pm 11.6 & \pm 4.5 & \pm 5.3 & \pm 4.7 & \pm 9.5 & \pm 6.2 & \pm 14.0 \\
\hline \multirow[t]{2}{*}{ Deckung der Streuschicht (\%) } & 39.7 & 40.0 & 51.7 & 42.1 & 32.4 & 60.0 & 46.4 & 49.1 & 44.0 & 22.4 \\
\hline & \pm 25.7 & \pm 17.5 & \pm 12.7 & \pm 11.8 & \pm 17.2 & \pm 9.2 & \pm 12.6 & \pm 12.1 & \pm 17.9 & \pm 15.7 \\
\hline \multirow[t]{2}{*}{ Deckung Totholz (\%) } & 5.4 & 9.2 & 12.7 & 16.6 & 17.6 & 16.9 & 14.8 & 20.5 & 14.6 & 1.1 \\
\hline & \pm 5.2 & \pm 6.8 & \pm 11.0 & \pm 8.0 & \pm 9.8 & \pm 2.6 & \pm 8.9 & \pm 8.2 & \pm 7.0 & \pm 1.8 \\
\hline \multirow[t]{2}{*}{ Offener Boden (\%) } & 32.2 & 36.1 & 22.7 & 23.3 & 15.4 & 14.4 & 21.4 & 13.3 & 19.8 & 31.6 \\
\hline & \pm 28 & \pm 19.7 & \pm 14.4 & \pm 15.8 & \pm 13.2 & \pm 9.0 & \pm 13.1 & \pm 10.3 & \pm 15.2 & \pm 20.6 \\
\hline \multirow{2}{*}{ Deckung Baumschicht(\%) } & 4.3 & 6.4 & 23.3 & 3.8 & 2.6 & 46.3 & 31.9 & 46.7 & 55.2 & 31.9 \\
\hline & \pm 9.8 & \pm 11.8 & \pm 24.3 & \pm 8.9 & \pm 7.5 & \pm 17.7 & \pm 24.9 & \pm 20.6 & \pm 18.4 & \pm 24.2 \\
\hline \multirow[t]{2}{*}{ Deckung Strauchschicht (\%) } & 50.0 & 49.8 & 45.0 & 40.0 & 42.6 & 48.1 & 55.0 & 47.7 & 48.7 & 12.5 \\
\hline & \pm 17.3 & \pm 28 & \pm 18.1 & \pm 20.2 & \pm 22.7 & \pm 12.5 & \pm 16.4 & \pm 13.5 & \pm 12.5 & \pm 10.8 \\
\hline \multirow[t]{2}{*}{ Deckung Krautschicht (\%) } & 38.9 & 42.1 & 57.9 & 32.4 & 32.1 & 34.4 & 61.8 & 62.3 & 66.3 & 64.1 \\
\hline & \pm 21.9 & \pm 24.5 & \pm 19.7 & \pm 22.4 & \pm 16.3 & \pm 18.8 & \pm 19.6 & \pm 18.7 & \pm 19 & \pm 20.5 \\
\hline \multirow[t]{2}{*}{ Max. Höhe Baumschicht (m) } & & 2.3 & 3.3 & & & 6.4 & 4.7 & 6.9 & 8 & 6.6 \\
\hline & & \pm 2.8 & \pm 3 & & & \pm 0.7 & \pm 2.4 & \pm 2 & \pm 1.6 & \pm 3.1 \\
\hline \multirow[t]{2}{*}{ Max. Höhe Strauchschicht (m) } & 3.8 & 3.6 & 3.4 & 3.7 & 3.6 & 3.7 & 3.6 & 3.4 & 3.8 & 2.2 \\
\hline & \pm 0.5 & \pm 0.7 & \pm 0.5 & \pm 0.5 & \pm 0.5 & \pm 0.5 & \pm 0.3 & \pm 0.4 & \pm 0.4 & \pm 1.2 \\
\hline \multirow[t]{2}{*}{ Max. Höhe Krautschicht (m) } & 1.1 & 0.9 & 1.0 & 1.0 & 1.0 & 1.1 & 1.2 & 1.3 & 1.5 & 1.3 \\
\hline & \pm 0.5 & \pm 0.3 & \pm 0.3 & \pm 0.3 & \pm 0.1 & \pm 0.3 & \pm 0.3 & \pm 0.2 & \pm 0.2 & \pm 0.3 \\
\hline
\end{tabular}

\section{Baumschicht}

Parkinsonia aculeata

Copernicia alba

Prosopis ruscifolia

32

$35 \quad 38$

Tabebuia nodosa

$326 \quad 59$

Caesalpinia paraguariensis

Salta triflora

Ziziphus mistol

Bougainvillea campanulata

Prosopis rojasiana

Piptadeniopsis lomentifera

Quiabentia verticillata

Cereus forbesii

Lycium boerhaaviaefolium

Prosopis hassleri

Bulnesia sarmientoi

Prosopis sericantha

Phyllostylon rhamnoides

Trithrinax schyzophylla

Aspidosperma quebracho-blanco

Acacia praecox

Sideroxylon obtusifolium

Cynophalla retusa

Cordia americana

Ximenia americana

Stetsonia coryne

Ceiba chodatii

Prosopis kuntzei

Geoffroea spinosa

58
43 
Tab. 5-1 (Fortsetzung) 10

111

Strauchschicht
Parkinsonia aculeatc

Funastrum clausum

Copernicia alba

Prosopis ruscifolia

Lycium nodosum

Solanum glaucophyllum

Passiflora mooreana

Acacia caven

Prosopis nigra

Lycium morongii

Capparicordis tweediana

Cercidium praecox

Coccoloba cordata

Prosopis hassleri

Tabebuia nodosa

Atriplex eximia

Prosopis rojasiana

Maytenus vitis-idaea

Lycium americanum

Lophocarpinia aculeatifolia

Lycium boerhaaviaefolium

Cyclolepis genistoides

Bulnesia bonariensis

Salta triflora

Cynophalla retusa

Bougainvillea campanulata

Harrisia bonplandii

Caesalpinia paraguariensis

Basistemon spinosus

Achatocarpus praecox

Aspidosperma quebracho-blanco

Acacia praecox

Sarcotoxicum salicifolium

Marsdenia castillonii

Ziziphus mistol

Piptadeniopsis lomentifera

Cereus forbesii

Acanthosyris falcata

Holmbergia tweedii

Bulnesia sarmientoi

Trithrinax schyzophylla

Celtis ehrenbergiana

Sideroxylon obtusifolium

Anisocapparis speciosa

Fridericia dichotoma

Phyllostylon rhamnoides

Herreria montevidensis

Schaefferia argentinensis

Pisonia hassleriana

Ximenia americana

$93 \quad 3$

$59 \quad 3$

31

5
30
31
25
25
4$$
\begin{array}{r}
7 \\
40
\end{array}
$$$$
40
$$$$
25
$$$$
22
$$ 
Tab. 5-1 (Fortsetzung) 10 11 12 13 14

\section{Krautschich}

76

Euphorbia serpens $\quad 65$

Neptunia pubescens 65

Funastrum clausum $\quad 50$

Copernicia alba 50

Solanum glaucophyllum $\quad 50$

Sesbania virgata 45

Setaria geminata $\quad 50$

Leptochloa fusca subsp. uninervia $\quad 44$

Cynodon niemfuensis 32

Amaranthus hybridus 32

Solanum sisymbriifolium $\quad 32$

Eclipta prostrata $\quad 32$

Solanum multispinum $\quad 32$

Amaranthus viridis $\quad 29$

Glinus radiatus 23

Chenopodium pilcomayense 23

Portulaca grandiflora 22

Cynodon dactylon 23

Heliotropium curassavicum 66

Alternanthera paronychioides subsp.

chacoese $\quad 30 \quad 33$

Lycium nodosum

Prosopis ruscifolia

Sporobolus pyramidatus

Sesuvium portulacastrum

Atriplex eximia

Sarcocornia ambigua

Euphorbia lasiocarpa

Stemodia ericifolia

Grindelia pulchella

Cuscuta species

Spergula ramosa

Lycium boerhaaviaefolium

Desmanthus virgatus

Evolvulus convolvuloides

Acacia caven

Ayenia odonellii

Priva boliviana

Prosopis hassleri

Melochia ministella

Senna morongii

Serjania marginata

Araujia herzogii

Cienfuegosia drummondii

Galactia latisiliqua

Mascagnia brevifolia

Melochia parvifolia

Spilanthes paraguayensis

Heliotropium pallescens

Mimosa detinens

Justicia dumetorum

Tabebuia nodosa

Ruellia simplex

Galactia longifolia

Vigna luteola

Polycarpon suffruticosum

Cynophalla retusa

Sida anomala 
Tab. 5-1 (Fortsetzung) 10

Baccharis salicifolia

Aspidosperma quebracho-blanco

Rivina humilis

Capparicordis tweediana

Cyperus corymbosus var. subnodosus

Pterocaulon purpurascens

Sclerophylax spinescens

Talinum paniculatum

Lycium americanum

Opuntia elata var. cardiosperma

Trichloris crinita

Ziziphus mistol

Cleistocactus baumannii

Echinopsis rhodotricha

Tillandsia mereliana

Heterostachys ritteriana

Cyclolepis genistoides

Bulnesia bonariensis

Dyckia ferox

Opuntia anacantha var. retrorsa

Deinacanthon urbanianum

Monvillea cavendischii

Trithrinax schyzophylla

Acacia praecox

Setaria nicorae

Ruellia hygrophila

Aechmea distichantha var. schlumbergeri

Harrisia bonplandii

Marsdenia castillonii

Ipomoea bonariensis

Sida cabreriana

Cyperus species

Coccoloba spinescens

$\begin{array}{llllllll}\mathbf{1 2} & \mathbf{1 3} & \mathbf{1 4} & \mathbf{1 5} & \mathbf{1 6} & \mathbf{1 7} & \mathbf{1 8} & \mathbf{1 9} \\ 16 & & & & & & 20 & \\ 22 & & & 7 & 2 & & 20 & \\ 21 & & & & & & 32 & \\ 22 & & & & 7 & 23 & 32 & \end{array}$

Carlowrightia sulcata

Opuntia colubrina

Selaginella sellowii

Piptadeniopsis lomentifera

Basistemon spinosus

Lippia alba

Bromelia hieronymi

1

29

24

$$
3
$$

$\begin{array}{ll}31 & 19\end{array}$

$16 \quad 47$

4

Bromelia serra

Gouinia paraguayensis

Justicia xylosteoides

Monvillea spegazzinii

Bougainvillea campanulata

Jacaratia corumbensis

Fridericia truncata

Celtis ehrenbergiana

Phyllostylon rhamnoides

Tragia hieronymii

Ruellia erythropus

Croton hieronymi

Ditaxis montevidensis

Sida dictyocarpa

Herreria montevidensis

Anisocapparis speciosa

Tournefortia rubicunda

Erythroxylum cuneifolium

Turnera weddelliana

Croton gracilipes

Oxalis frutescens 
Tab. 5-1 (Fortsetzung)

10

$11 \quad 12$

1213

Cardiospermum halicacabum

Capsicum chacoense

Lantana balansae

Achatocarpus praecox

Lasiacis sorghoidea

Salta triflora

Castela coccinea

Serjania species

Eupatorium christieanum

Justicia squarrosa

Leptochloa virgata

Sideroxylon obtusifolium

Staelia virgata

Megathyrsus maximus

Fridericia dichotoma

Aristida mendocina

Cnidoscolus albomaculatus

Pappophorum krapovickasii

Rhynchosia burkartii

Melochia werdermannii

Acacia aroma

Gomphrena perennis

Elionurus muticus

Urochloa paucispicata

$9 \quad 32$

$18 \quad 31$

Chamaecrista serpens

Mimosa debilis

Scoparia dulcis

Jacquemontia decumbens

Bauhinia argentinensis

Zornia latifolia

Schinopsis cornuta

Tabebuia aurea

Microstachys hispida

Porophyllum ruderale

Ayenia eliae

Astraea lobata

Aeschynomene viscidula

Evolvulus sericeus

Chamaecrista arachiphylla

Croton glandulosus

Borreria eryngioides

Cleome tucumanensis

Turnera krapovickasii

Pseudabutilon virgatum

Pavonia vannii

Astronium fraxinifolium

Salvia cardiophylla

Malvastrum coromandelianum

Vernonia niederleini

Eragrostis orthoclada

Zexmenia brachylepis

Pavonia hieronymi

Cienfuegosia argentina

Cyperus aggregatus

Aeschynomene histrix

Waltheria indica

Heliotropium dunaense

Bulbostylis capillaris

Digitaria species

Eupatorium patens

Dyschoriste venturii 
Tab. 5-1 (Fortsetzung)

Dicliptera squarrosa

Passiflora foetida

Janusia guaranitica

Croton bonplandianus

Herissantia crispa

Noticastrum acuminatum

Pterocaulon virgatum

Turnera grandiflora

Chloris ciliata

Mimosa xanthocentra

Waltheria communis

Aspilia pascalioides

Macroptilium bracteatum

Heliotropium campestre

Boerhavia diffusa

Schizachyrium species

Wedelia species

Abildgaardia ovata

Oxypetalum arnottianum

Desmodium incanum

Eupatorium catarium

Gaya tarijensis

Jatropha excisa

Schwenckia americana

\section{Epiphyten}

Phoradendron argentinum

Psittacanthus cordatus

Tillandsia mereliana

Tillandsia duratii var. saxatilis

Tillandsia recurvifolia

Selenicereus setaceus

Tillandsia loliacea

Tillandsia reichenbachii

Microgramma vacciniifolia

Rhipsalis baccifera
10

11

12

13

14

15

16

17

1

18

19

1

26

4

$12 \quad 26$

$2 \quad 14 \quad 9$

$45 \quad 5$

25

\begin{tabular}{|c|c|c|c|c|}
\hline \multirow{2}{*}{$\begin{array}{l}44 \\
31\end{array}$} & \multicolumn{3}{|c|}{8} & \\
\hline & & 12 & 32 & 4 \\
\hline \multirow{5}{*}{4} & 43 & 7 & 5 & 10 \\
\hline & 43 & & & 14 \\
\hline & 25 & 33 & 19 & 24 \\
\hline & & 43 & & 18 \\
\hline & & 8 & & 42 \\
\hline
\end{tabular}




\subsection{2. Ökologische Eigenschaften der Gesellschaften}

Die untersuchten Gehölzgesellschaften sind entlang eines Wasserverfügbarkeitsgradienten angeordnet. Entlang dieses Gradienten spielt die Bodenart im Oberboden, wie in der Abbildung 5-12 ersichtlich ist, eine entscheidende Rolle. Hohe Schluff- und Tonanteile im Oberboden führen zu einer schlechten Dränage und somit zu stauwasserbeeinflussten Habitaten. Dieser Effekt kann ganz besonders an Standorten der Parkinsonia aculeataCopernicia alba-Gesellschaft (10), der Prosopis ruscifolia-Sporobolus pyramidatusGesellschaft (11), der Prosopis ruscifolia-Evolvulus convolvuloides-Gesellschaft (12) und der Prosopis ruscifolia-Tabebuia nodosa-Gesellschaft (15) beobachtet werden.

Die Habitate der Prosopis ruscifolia-Lycium americanum- (13) und der Cyclolepis genistoides-Lycium americanum-Gesellschaften (14) werden besonders von hohen Natrium-, pH- und Leitfähigkeits-Werten im Boden charakterisiert. Geringere Salzmengen und ein vegetationsbeeinflusstes Mikroklima fördern an den Standorten der Waldgesellschaften (17 und 18) die Streuzersetzung und die damit verbundene Anreicherung organischer Substanz im Oberboden.

In einer direkten Gradientenanalyse (Abb. 5-13) konnten in den Strauch- und Waldgesellschaften fünf signifikante Bodenparameter ermittelt werden: Natrium in der unteren Bodenschicht (Na2, p-Wert=0.002), Sand in der oberen Bodenschicht (Sand1, pWert=0.012), $\mathrm{pH}$ in der oberen Bodenschicht ( $\mathrm{pH} 1, \mathrm{p}-$ Wert=0.028), organische Substanz in der oberen Bodenschicht (Org1, p-Wert $=0,034$ ) und Schluff in der unteren Bodenschicht (Schluff2, p-Wert=0,05). Wie auch in der indirekten Gradientenanalyse, sind die Sand- und Schluff-Anteile im Oberboden negativ korreliert und führen zu unterschiedlichen Standorteigenschaften und Artenzusammensetzungen.

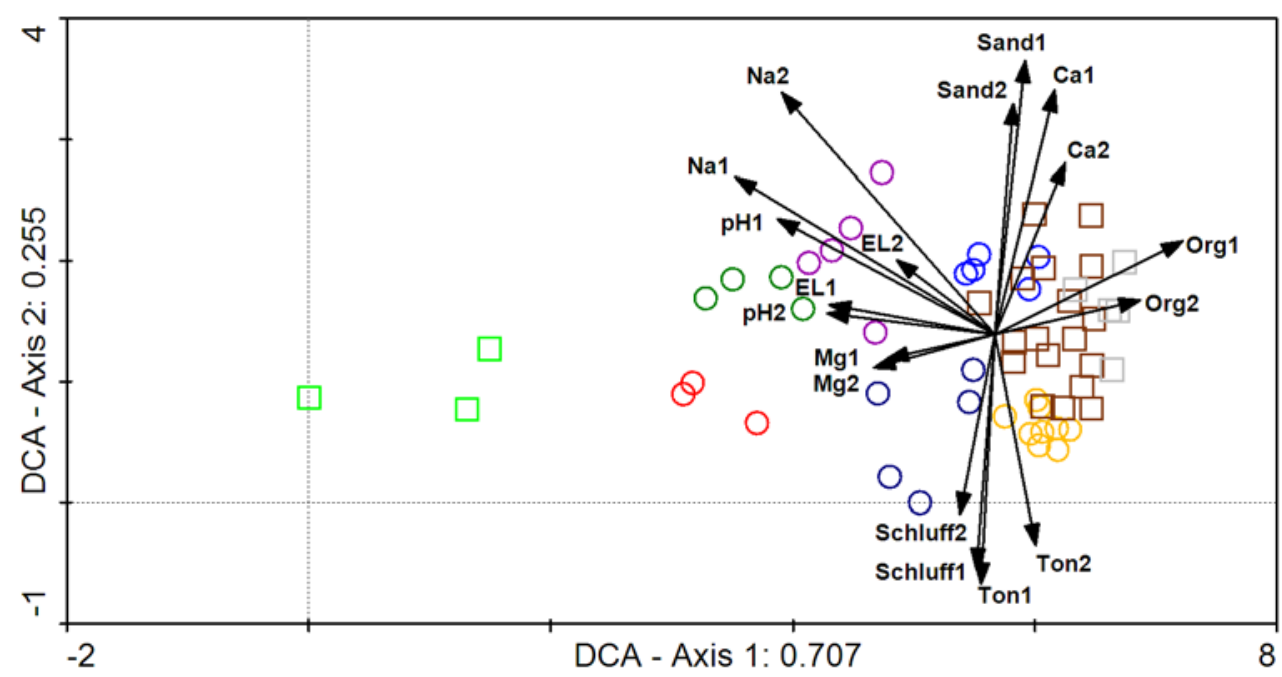

Abb. 5-12: DCA - Ordinationsdiagramm der Gehölzgesellschaften 10 - 18 in einer indirekten Gradientenanalyse mit den Bodenparametern. Abgebildet werden die Probeflächen, von denen Bodenproben genommen wurden, und die Bodenparameter: Sand, Schluff, Ton, pH, Natrium (Na), Magnesium (Mg), Calcium (Ca) und Organische Masse (Org) in der oberen (1) und unteren (2) Bodenschicht. Die abgebildeten Pflanzengesellschaften sind: $\square$ Einheit 10, $\bigcirc$ Einheit 11, $\bigcirc$ Einheit 12, $\bigcirc$ Einheit 13, OEinheit 14, $\bigcirc$ Einheit 15, $\bigcirc$ Einheit 16, $\square$ Einheit 17, $\square$ Einheit 18, Bodenparameter (Log-file-Daten im Anhang 5-6) 

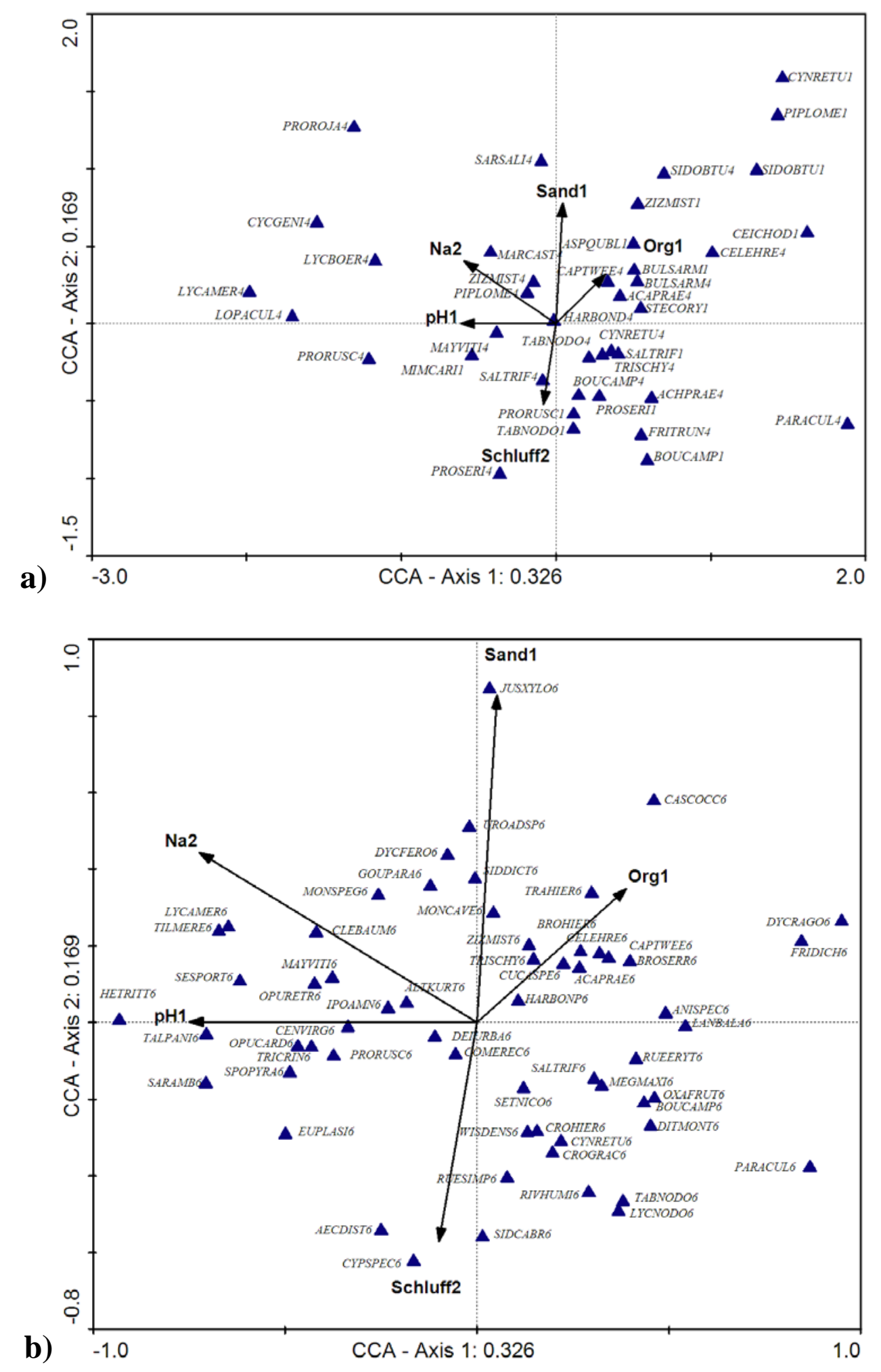

Abb. 5-13: CCA - Ordinationsdiagramme der signifikanten Bodenparameter. Abgebildet werden die Arten der Baum- und Strauchschicht (a) und der Krautschicht (b) (Gesellschaften 10 - 18) mit den fünf signifikanten Bodenparametern: Natrium in der unteren Bodenschicht $(\mathrm{Na} 2$, p-Wert=0.002), Sand in der oberen Bodenschicht (Sand1, p-Wert=0.012), pH in der oberen Bodenschicht (pH1, p-Wert=0.028), Schluff in der unteren Bodenschicht (Schluff2, p-Wert=0,05) und organische Substanz in der oberen Bodenschicht (Org1, pWert=0,034). $\mathbf{\Delta}$ zeigt die Position der jeweiligen Arten (Artnamen zu den jeweiligen Abkürzungen im Anhang 5-2; Log-file-Daten im Anhang 5-7) 
Hohe Schluffanteile im unteren Horizont des Oberbodens (Abb. 5-13) führen zur schwachen Dränage und Pseudovergleyung. Typische Arten in der Strauch- und Baumschicht an diesen Standorten sind Tabebuia nodosa, Prosopis ruscifolia, Parkinsonia aculeata, und Prosopis sericantha. In der Krautschicht treten häufig Ruellia simplex, Aechmea distichantha var. schlumbergeri, Lycium nodosum und Rivina humilis auf.

Standorte im Untersuchungsgebiet mit hohem Sandanteil im Oberboden werden dagegen hauptsächlich von Ziziphus mistol, Sideroxylon obtusifolium, Sarcotoxicum salicifolium, Justicia xylosteoides, Sida dictyocarpa und Urochloa adspersa besiedelt.

Hohe Natrium- und pH-Werte im Oberboden sind in den untersuchen Probeflächen positiv korreliert und kennzeichnen salzreiche Standorte, die größtenteils von halophilen Arten besiedelt werden. Typische Straucharten sind Lycium americanum, Cyclolepis genistoides, Lophocarpinia aculeatifolia, Lycium boerhaaviaefolium und Prosopis rojasiana. In der Krautschicht kommen weitverbreitete Halophyten wie Sarcocornia ambigua, Heterostachys ritteriana, Sesuvium portulacastrum, Sporobolus pyramidatus und die in paraguayischen Salzstandorten endemische Tillandsia mereliana vor.

Standorte mit einer höheren Anreicherung an organischer Substanz im Oberboden stehen meist in Verbindung mit den Waldgesellschaften. Typische krautige Arten, die in diesen Habitaten wachsen, sind Bromelia hieronymi, Tragia hieronymi, Bromelia serra und Capparicordis tweediana.

Interessante Einblicke in die ökologischen Eigenschaften der Gesellschaften geben außerdem die vorkommenden Epiphyten (Tab. 5-1). Zwar konnten keine artspezifischen Zusammenhänge zwischen den vorkommenden Epiphyten und Porophyten beobachtet werden, doch können gewisse epiphytische Arten Auskunft über die Luftfeuchtigkeit am Standort geben. So kommen trockenliebende Tillandsien, wie T. reichenbachii und T. duratii var. saxatilis besonders häufig in xerohalophilen Strauchgebüschen vor, während Tillandsia mereliana kennzeichnend für die xerohalophile Cyclolepis genistoides-Lycium americanumGesellschaft ist. Feuchtigkeitsliebende Epiphyten, wie Rhipsalis baccifera, Selenicereus setaceus und Microgramma vaccinifolia kommen dagegen hauptsächlich in feuchten Wäldern vor. Diese drei Arten sind besonders häufig im Osten des Untersuchungsgebietes anzutreffen und zeigen somit eine klare Verbindung zum feuchten Chaco.

Auf gewissen Baum- und Straucharten, wie die in mehreren Gesellschaften dominierende Prosopis ruscifolia, kommen keine oder nur selten Epiphyten vor. Prosopis ruscifolia wird dagegen häufig von Hemiparasiten, wie Phoradendron argentinum und Psittacanthus cordatus besiedelt.

\subsubsection{Strukturelle Zusammensetzung der Gesellschaften}

Bei den Strukturmessungen im Untersuchungsgebiet wurden nur Stämme mit einem Brusthöhendurchmesser (BHD) $\geq 10 \mathrm{~cm}$ berücksichtigt. Die gemessenen Gehölze gehören zu 45 Arten, 36 Gattungen und 20 Familien. Der Datensatz beinhaltet $93 \%$ lebend-stehende, 2 \% lebend-umgefallene und $5 \%$ trocken-stehende Stämme. 72 \% der gemessenen Gehölze sind einstämmig, 15 \% zweistämmig, 6 \% dreistämmig und 7 \% der Individuen bestehen aus vier oder mehr Teilstämmen. 


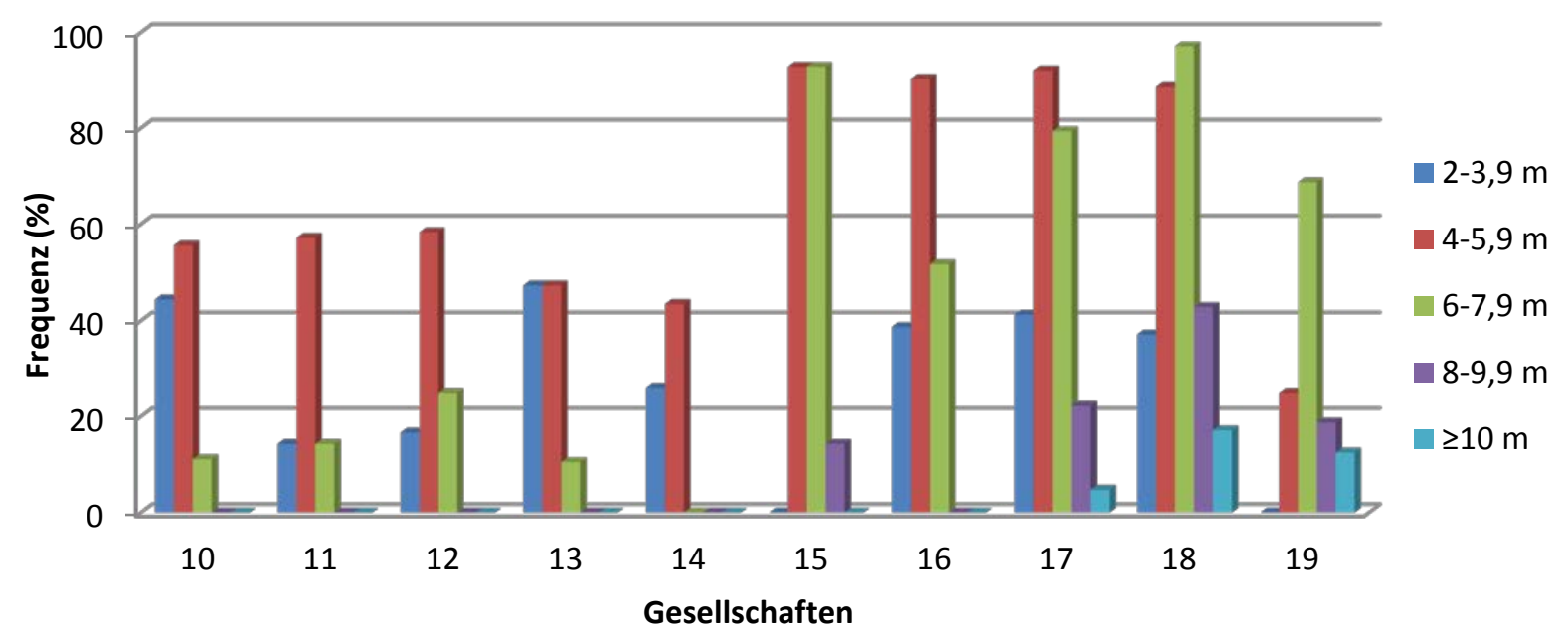

Abb. 5-14: Absolute Frequenz der Höhen-Verteilung in den jeweiligen Gesellschaften (Gehölze mit einem BHD $\geq 10 \mathrm{~cm}$ ). Die absolute Frequenz (\%) zeigt das Auftreten bzw. Fehlen der jeweiligen Höhenklassen in den einzelnen Probeflächen jeder Gesellschaft.

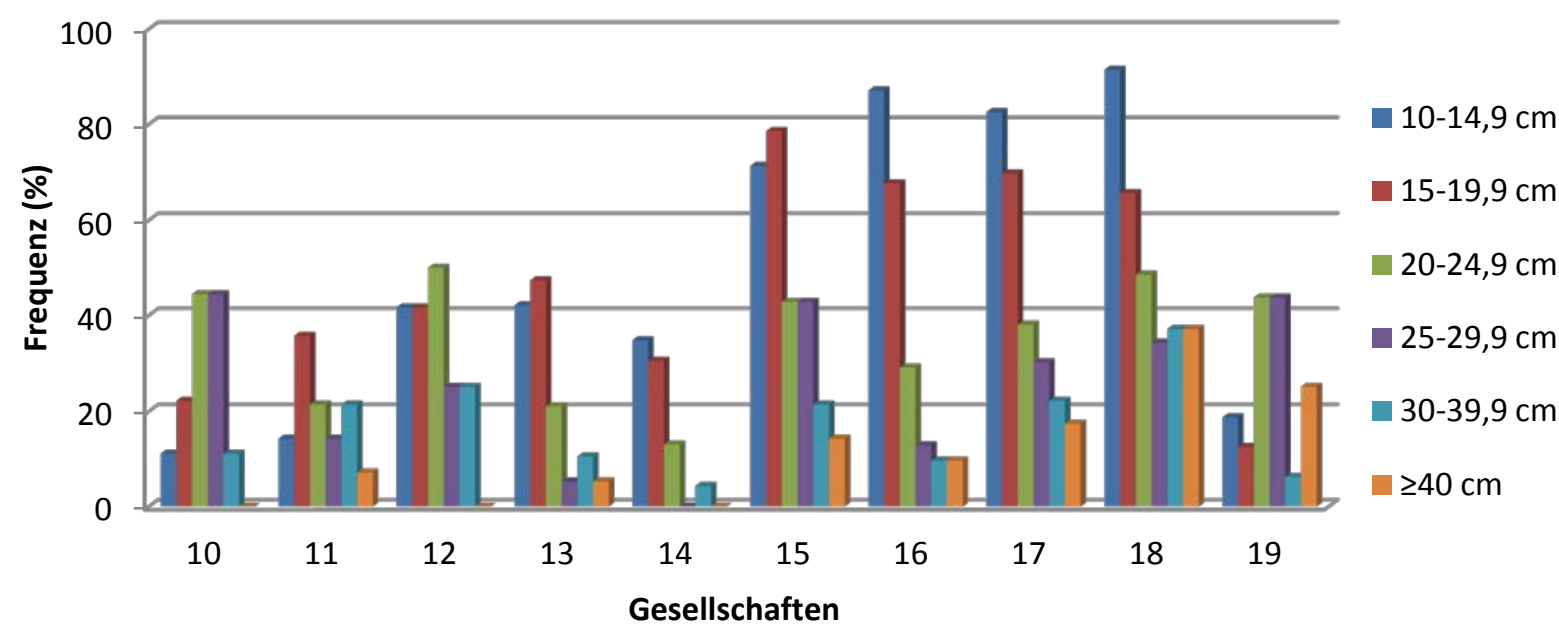

Abb. 5-15: Absolute Frequenz der BHD-Verteilung in den jeweiligen Gesellschaften (Gehölze mit einem BHD $\geq 10 \mathrm{~cm}$ ). Die absolute Frequenz (\%) zeigt das Auftreten bzw. Fehlen der jeweiligen BHD-Klassen in den einzelnen Probeflächen jeder Gesellschaft.

Rund 80 \% der untersuchten Gehölze erreichen eine Höhe von 4 - 7,9 m. In den Gebüschgesellschaften (Einheiten 10 - 14) sind Gehölze mit Höhen von 4-5,9 m häufig, während in den Waldgesellschaften (Einheiten 15 - 18) die Gehölze mit Höhen von 4 - 7,9 m die Vegetationsstruktur besonders prägen (Abb. 5-14). Gehölze, die Höhen unter $4 \mathrm{~m}$ erreichen, spielen in dieser Studie eine untergeordnete Rolle da viele Sträucher in den Gesellschaften nicht einen BHD von $10 \mathrm{~cm}$ erreichen. Höhenstufen über $8 \mathrm{~m}$ spielen hauptsächlich in den höheren Waldgesellschaften (Einheiten 17 und 18) und in der Baumsavanne (19) eine Rolle.

Auffallend sind die deutlich höheren Frequenzen der einzelnen Strukturverteilungen, sowohl der Höhe (Abb. 5-14) als auch des BHD (Abb. 5-15), in den Waldgesellschaften. Gründe 
dafür liegen sowohl in der höheren Arten- und Strukturvielfalt der Waldgesellschaften als auch in der artenarmen und niedrigwüchsigen Struktur der Strauchgesellschaften.

71 \% der untersuchten Gehölze haben einen BHD von 10 - 19,9 cm. In allen Gesellschaften, mit Ausnahme der Einheiten 10 und 19, weisen die ersten beiden BHD-Verteilungen die höchsten Frequenzen auf. In den Parkinsonia aculeata-Copernicia alba- (10) und Schinopsis cornuta-Elionurus muticus-Gesellschaften (19) weisen dagegen Gehölze mit BHD 20-29,9 cm die höchsten Frequenzen auf. Bei der ersten dürfte dieses auf die hohe Mehrstämmigkeit der Parkinsonia aculeata und der Palmstämme der Copernicia alba hindeuten, während es sich bei der Einheit 19 um eine Baumsavanne mit einzelnen höheren Bäumen handelt. Brusthöhendurchmesser von mehr als $40 \mathrm{~cm}$ spielen hauptsächlich in den höheren Waldgesellschaften (Einheiten 17 und 18) und der Baumsavanne (Einheit 19) eine Rolle.

Die Abundanzwerte der Gehölze in den einzelnen Gesellschaften (Tab. 5-2) werden hauptsächlich von der Arten- und Strukturvielfalt geprägt. In Gesellschaften mit artenarmer Gehölzstruktur (Einheiten 10 bis 14 und 19) erreichen einige dominante Arten besonders hohe Abundanzwerte. Die Gesellschaften weisen sowohl niedrige Gesamtabundanz- als auch Gesamtdominanz-Werte auf. Die artenreichen Waldgesellschaften (Einheiten 17 und 18) dagegen weisen hohe Gesamtabundanz- und Gesamtdominanz-Werte auf. Arten mit den höchsten Abundanzwerten im Untersuchungsgebiet insgesamt sind Prosopis ruscifolia und Ruprechtia triflora. Während Prosopis ruscifolia besonders hohe Abundanzen in den Gesellschaften 11 bis 15 erreicht, kommt Salta triflora besonders häufig in den Gesellschaften 15 bis 18 vor.

Der Bedeutungswert-Index (IVI), der sich aus der relativen Abundanz, Frequenz und Dominanz der Gehölzarten in den Gesellschaften zusammensetzt, wird in der Tabelle 5-3 gezeigt. Prosopis ruscifolia weist auch hier extrem hohe Werte auf. Insbesondere in den Gesellschaften 11 bis 14 erreicht diese Art sehr hohe IVI-Werte. In der Einheit 10 erreichen Parkinsonia aculeata und Copernicia alba hohe IVI-Werte. Allerdings liegen diesem Ergebnis nur neun Probeflächen zugrunde.

In der Baumsavanne auf Sandböden (Einheit 19) weisen alle drei vorkommenden Bäume hohe IVI-Werte auf. In der Prosopis ruscifolia-Tabebuia nodosa-Gesellschaft werden Tabebuia nodosa, Prosopis ruscifolia und Salta triflora mit einem hohen BewertungswertIndex charakterisiert. Gesellschaften mit einer höheren Gehölzvielfalt, wie die Einheiten 16 bis 18, weisen dagegen meist niedrige IVI-Werte auf. Die bedeutendsten Arten in der Einheit 16 sind Salta triflora, Ceiba chodatii und Ziziphus mistol. In der Einheit 17 weisen Bulnesia sarmientoi, Salta triflora und Ziziphus mistol und in der Einheit 18 Salta triflora, Ceiba chodatii und Trithrinax schizophylla bedeutende IVI-Werte auf. 
Tab. 5-2: Absolute Abundanz und absolute Dominanz der Gehölzarten mit einem BHD $\geq 10 \mathrm{~cm}$ in den jeweiligen Vegetationseinheiten

\begin{tabular}{|c|c|c|c|c|c|c|c|c|c|c|c|c|c|c|c|c|c|c|c|c|}
\hline \multirow[b]{2}{*}{ Art\Vegetationseinheit } & \multicolumn{9}{|c|}{ Abundanz (Individuen/ha) } & \multicolumn{11}{|c|}{ Dominanz $\left(m^{2} / h a\right)$} \\
\hline & $10 *$ & 11 & 12 & 13 & 14 & 15 & 16 & 17 & 18 & 19 & $10 *$ & 11 & 12 & 13 & 14 & 15 & 16 & 17 & 18 & 19 \\
\hline Parkinsonia aculeata & 111 & 7 & & & & & & & & & 4.84 & 0.12 & & & & & & & & \\
\hline Copernicia alba & 67 & 43 & 8 & & & & & & & & 2.43 & 1.06 & 0.34 & & & & & & & \\
\hline Prosopis ruscifolia & & 121 & 275 & 174 & 87 & 100 & 16 & 16 & & & & 6.96 & 9.63 & 5.12 & 2.35 & 5.73 & 0.49 & 0.54 & & \\
\hline Salta triflora & & & & & 4 & 157 & 132 & 116 & 163 & & & & & & 0.04 & 2.13 & 1.70 & 1.45 & 2.45 & \\
\hline Tabebuia nodosa & & & 25 & 5 & & 129 & 10 & 43 & 17 & & & & 0.65 & 0.05 & & 6.74 & 0.42 & 1.22 & 0.43 & \\
\hline Bulnesia sarmientoi & & & & 5 & & 7 & 16 & 117 & 26 & & & & & 0.10 & & 0.11 & 0.43 & 6.58 & 3.14 & \\
\hline Trithrinax schizophylla & & & & & & 7 & 55 & 79 & 111 & & & & & & & 0.08 & 0.61 & 0.82 & 1.06 & \\
\hline Ziziphus mistol & & & & & 4 & & 68 & 73 & 34 & & & & & & 0.08 & & 1.39 & 1.85 & 0.90 & \\
\hline Prosopis rojasiana & & & & & 17 & 7 & 6 & 2 & & & & & & & 0.30 & 0.10 & 0.24 & 0.05 & & \\
\hline Sideroxylon obtusifolium & & & & & & & 29 & 17 & 57 & & & & & & & & 0.87 & 0.47 & 2.21 & \\
\hline Bougainvillea campanulata & & & & & & & 26 & 16 & 23 & & & & & & & & 0.71 & 0.21 & 0.60 & \\
\hline Ceiba chodatii & & & & & & & 26 & 10 & 31 & & & & & & & & 5.79 & 3.05 & 13.05 & \\
\hline Aspidosperma quebracho-blanco & & & & & & & 10 & 6 & 46 & & & & & & & & 0.24 & 0.15 & 2.53 & \\
\hline Phyllostylon rhamnoides & & & & & & & & 5 & 66 & & & & & & & & & 0.10 & 1.72 & \\
\hline Acacia praecox & & & & & & & 3 & 6 & 20 & & & & & & & & 0.03 & 0.14 & 0.39 & \\
\hline Stetsonia coryne & & & & & & & 13 & 8 & 17 & & & & & & & & 0.37 & 0.41 & 0.63 & \\
\hline Caesalpinia paraguayensis & & & & & & 14 & & & 3 & & & & & & & 0.27 & & & 0.13 & \\
\hline Cordia americana & & & & & & & & & 14 & & & & & & & & & & 1.52 & \\
\hline Cynophalla retusa & & & & & & & 3 & & 14 & & & & & & & & 0.03 & & 0.22 & \\
\hline Ximenia americana & & & & & & & 3 & & 17 & & & & & & & & 0.05 & & 0.51 & \\
\hline Maytenus vitis-idaea & & & & & & & 16 & 6 & & & & & & & & & 0.25 & 0.11 & & \\
\hline Mimozyganthus carinatus & & & & & 9 & & 6 & 6 & & & & & & & 0.23 & & 0.50 & 0.26 & & \\
\hline Prosopis hassleri & & & & & & & 3 & & & & & & & & & & 0.35 & & & \\
\hline Prosopis sericantha & & & & & & & 3 & 6 & & & & & & & & & 0.04 & 0.09 & & \\
\hline Quiabentia verticillata & & & & & 4 & & 3 & & & & & & & & 0.08 & & 0.03 & & & \\
\hline Sarcotoxicum salicifolium & & & & & & & 3 & 3 & & & & & & & & & 0.11 & 0.10 & & \\
\hline Cercidium praecox & & & & & & & & 3 & & & & & & & & & & 0.03 & & \\
\hline
\end{tabular}


Tab. 5-2 (Fortsetzung)

\begin{tabular}{|c|c|c|c|c|c|c|c|c|c|c|c|c|c|c|c|c|c|c|c|c|}
\hline \multirow[b]{2}{*}{ Art \Vegetationseinheit } & \multicolumn{10}{|c|}{ Abundanz (Individuen/ha) } & \multicolumn{10}{|c|}{ Dominanz $\left(\mathrm{m}^{2} / \mathrm{ha}\right)$} \\
\hline & $10 *$ & 11 & 12 & 13 & 14 & 15 & 16 & 17 & 18 & 19 & $10^{*}$ & 11 & 12 & 13 & 14 & 15 & 16 & 17 & 18 & 19 \\
\hline Chloroleucon tenuiflorum & & & & & & & & 2 & & & & & & & & & & 0.02 & & \\
\hline Lycium boerhaaviaefolium & & & & & & & 3 & & & & & & & & & & 0.11 & & & \\
\hline Cereus forbesii & & & & & & 7 & & 2 & & & & & & & & 0.13 & & 0.02 & & \\
\hline Piptadeniopsis lomentifera & & & & & & & 6 & 2 & 6 & & & & & & & & 0.07 & 0.04 & 0.08 & \\
\hline Acanthosyris falcata & & & & & & & 3 & & 3 & & & & & & & & 0.04 & & 0.03 & \\
\hline Prosopis kuntzei & & & & & & & & 3 & 9 & & & & & & & & & 0.12 & 1.03 & \\
\hline Pisonia zapallo & & & & & & & & & 6 & & & & & & & & & & 0.19 & \\
\hline Aspidosperma triternatum & & & & & & & & & 6 & & & & & & & & & & 0.05 & \\
\hline Cordia glabrata & & & & & & & & & 6 & & & & & & & & & & 0.11 & \\
\hline Diplokeleba floribunda & & & & & & & & & 6 & & & & & & & & & & 0.09 & \\
\hline Anisocapparis speciosa & & & & & & & & & 3 & & & & & & & & & & 0.02 & \\
\hline Cereus stenogonus & & & & & & & & & 3 & & & & & & & & & & 0.19 & \\
\hline Coccoloba cordata & & & & & & & & & 3 & & & & & & & & & & 0.04 & \\
\hline Microlobius foetidus & & & & & & & & & 3 & & & & & & & & & & 0.10 & \\
\hline Schinopsis lorentzii & & & & & & & & & 3 & & & & & & & & & & 0.04 & \\
\hline Schinopsis cornuta & & & & & & & & & & 94 & & & & & & & & & & 4.26 \\
\hline Tabebuia aurea & & & & & & & & & & 38 & & & & & & & & & & 1.87 \\
\hline Astronium fraxinifolium & & & & & & & & & & 31 & & & & & & & & & & 4.74 \\
\hline TOTAL & 178 & 171 & 308 & 184 & 126 & 429 & 465 & 548 & 714 & 163 & 7.28 & 8.13 & 10.62 & 5.27 & 3.08 & 15.29 & 14.87 & 17.82 & 33.47 & 10.87 \\
\hline
\end{tabular}

* weniger als 10 Probeflächen 
Tab. 5-3: Bedeutungswert-Index der häufigsten 20 Gehölzarten im Untersuchungsgebiet

\begin{tabular}{|c|c|c|c|c|c|c|c|c|c|c|c|c|c|c|c|c|c|c|c|c|c|c|c|c|c|c|c|c|c|c|c|c|c|c|c|c|c|c|}
\hline \multirow[b]{2}{*}{ Art\Vegeationseinheit } & \multicolumn{9}{|c|}{ Relative Dominanz (\%) } & \multicolumn{10}{|c|}{ Relative Frequenz (\%) } & \multicolumn{9}{|c|}{ Relative Abundanz (\%) } & \multicolumn{10}{|c|}{ Bedeutungswert-Index (IVI)(\%) } \\
\hline & $10 *$ & 11 & 12 & 13 & 14 & 15 & 16 & 17 & $18 \quad 19$ & $10^{*}$ & 11 & 12 & 13 & 14 & 15 & 16 & 17 & 18 & 19 & $10 *$ & 11 & 12 & 13 & 14 & 15 & 161 & 171 & 1819 & $9 \mid 10^{*}$ & * 11 & 12 & 13 & 14 & 15 & 16 & 17 & 18 & 19 \\
\hline Parkinsonia aculeata & 67 & 1 & & & & & & & & 64 & 7 & & & & & & & & & 63 & 4 & & & & & & & & 193 & 13 & & & & & & & & \\
\hline Copernicia alba & 33 & 13 & 3 & & & & & & & 36 & 29 & 9 & & & & & & & & 38 & 25 & 3 & & & & & & & 107 & 67 & 15 & & & & & & & \\
\hline Prosopis ruscifolia & & 86 & 91 & 97 & 76 & 37 & 3 & 3 & & & 64 & 82 & 88 & 63 & 24 & 5 & 3 & & & & 71 & 89 & 94 & 69 & 23 & 3 & 3 & & & 221 & 262 & 280 & 208 & 85 & 11 & 9 & & \\
\hline Tabebuia nodosa & & & 6 & 1 & & 44 & 3 & 7 & 1 & & & 9 & 6 & & 30 & 3 & 9 & 4 & & & & 8 & 3 & & 30 & 2 & 8 & 2 & & & 23 & 10 & & 104 & 8 & 24 & 7 & \\
\hline Bulnesia sarmientoi & & & & 2 & & 1 & 3 & 37 & 9 & & & & 6 & & 3 & 2 & 20 & 4 & & & & & 3 & & 2 & 32 & 21 & 4 & & & & 11 & & 5 & 9 & 79 & 17 & \\
\hline Salta triflora & & & & & 1 & 14 & 11 & 8 & 7 & & & & & 6 & 30 & 17 & 16 & 16 & & & & & & 3 & 372 & 282 & 212 & 23 & & & & & 11 & 80 & 57 & 45 & 46 & \\
\hline Ziziphus mistol & & & & & 3 & & 9 & 10 & 3 & & & & & 6 & & 15 & 12 & 6 & & & & & & 3 & & 151 & 13 & 5 & & & & & 12 & & 39 & 36 & 14 & \\
\hline Prosopis rojasiana & & & & & 10 & 1 & 2 & & & & & & & 13 & 3 & 1 & 1 & & & & & & & 14 & 2 & 1 & & & & & & & 36 & 5 & 4 & 1 & & \\
\hline Quiabentia verticillata & & & & & 3 & & & & & & & & & 6 & & 1 & & & & & & & & 3 & & 1 & & & & & & & 12 & & 2 & & & \\
\hline $\begin{array}{l}\text { Mimozyganthus } \\
\text { carinatus }\end{array}$ & & & & & 7 & & 3 & 1 & & & & & & 6 & & 2 & 2 & & & & & & & 7 & & 1 & 1 & & & & & & 20 & & 7 & 5 & & \\
\hline Trithrinax schizophylla & & & & & & 1 & 4 & 5 & 3 & & & & & & 3 & 5 & 10 & 9 & & & & & & & 21 & 121 & 141 & 16 & & & & & & 5 & 21 & 29 & 28 & \\
\hline Ceiba chodatii & & & & & & & 39 & 17 & 39 & & & & & & & 7 & 3 & 6 & & & & & & & & 6 & 2 & 4 & & & & & & & 52 & 22 & 49 & \\
\hline $\begin{array}{l}\text { Bougainvillea } \\
\text { campanulata } \\
\text { Sideroxylon }\end{array}$ & & & & & & & 5 & 1 & 2 & & & & & & & 8 & 4 & 5 & & & & & & & & 6 & 3 & 3 & & & & & & & 18 & 8 & 10 & \\
\hline $\begin{array}{l}\text { obtusifolium } \\
\text { Aspidosperma }\end{array}$ & & & & & & & 6 & 3 & 7 & & & & & & & 9 & 4 & 9 & & & & & & & & 6 & 3 & 8 & & & & & & & 21 & 10 & 24 & \\
\hline quebracho-blanco & & & & & & & 2 & 1 & 8 & & & & & & & 3 & 2 & 7 & & & & & & & & 2 & 1 & 6 & & & & & & & 7 & 4 & 21 & \\
\hline Maytenus vitis-idaea & & & & & & & 2 & 1 & & & & & & & & 6 & 2 & & & & & & & & & 3 & 1 & & & & & & & & 11 & 3 & & \\
\hline $\begin{array}{l}\text { Phyllostylon } \\
\text { rhamnoides }\end{array}$ & & & & & & & & 1 & 5 & & & & & & & & 2 & 8 & & & & & & & & & 1 & 9 & & & & & & & & 3 & 22 & \\
\hline Schinopsis cornuta & & & & & & & & & 39 & & & & & & & & & & 50 & & & & & & & & & 58 & 8 & & & & & & & & & 147 \\
\hline $\begin{array}{l}\text { Astronium } \\
\text { fraxinifolium }\end{array}$ & & & & & & & & & 44 & & & & & & & & & & 19 & & & & & & & & & 19 & 9 & & & & & & & & & 82 \\
\hline Tabebuia aurea & & & & & & & & & 17 & & & & & & & & & & 31 & & & & & & & & & 23 & 3 & & & & & & & & & 72 \\
\hline
\end{tabular}

* weniger als 10 Probeflächen 


\subsection{Diskussion}

\subsubsection{Vegetationseinheiten im regionalen Vergleich}

Die Vegetationsformation der „Vinalares“ mit Prosopis ruscifolia, wie sie von Morello \& Saravia (1959), Spichiger et al. (1991), Prado (1993a) und Mereles (2005) für den Gran Chaco beschrieben wird, ist besonders häufig im Untersuchungsgebiet anzutreffen. Die hier beschriebenen Einheiten 11, 12 und 15 können dieser Formation zugeordnet und zugleich als unterschiedliche Stadien einer Sukzessionsreihe verstanden werden. Vinalares kommen meist an Standorten mit hoher Bodenalkalinität und -salinität in der Nähe periodisch wasserführender Flusssysteme vor. Laut Morello \& Saravia (1959) sind sie aufgrund der artenarmen und offenen Krautschicht und des Pfahlwurzel-Systems des Prosopis ruscifolia besonders anfällig für Bodenerosion.

Die hygrohalophilen Prosopis ruscifolia-Gebüschgesellschaften (Einheiten 11 bis 13) sind in ihrer Artenzusammensetzung ähnlich der von Navarro (1997) und Navarro \& Maldonado (2002) beschriebenen Prosopis ruscifolia-Copernicia alba-Gesellschaft (Prosopidi ruscifoliae-Copernicietum albae) an feuchten und periodisch überschwemmten Salzstandorten im bolivianischen Chaco. Im Gegensatz zu den untersuchten Gebieten in denen die Palme Copernicia alba nur eine untergeordnete Rolle spielt, prägt diese Art besonders die Physiognomie der für Bolivien beschriebenen Gesellschaft. Es ist wahrscheinlich, dass auch im paraguayischen Chaco, jedoch in Gegenden des feuchten Ostchaco, diese Gesellschaft vorkommt.

Die Prosopis ruscifolia-Lycium americanum- (13) und die Lycium americanum- Cyclolepis genistoides-Gesellschaften (14) sind zusammen mit den Salzmarschen (Kapitel 4) als „Estepas halófitas“ (Spichiger et al. 1991), „Matorral de salinar“ (Huespe et al. 1991), „Matorral de saladar“, „Matorrales marginales de los riachos y lagunas saladas“ und „Sabanas de saladares“ (Mereles 2004, 2005, 2007) in einer Formation beschrieben worden. Die Formation steht in direkter Verbindung zu stark salzhaltigen und natriumreichen Oberböden.

In Argentinien wird sowohl für den Chaco (Lewis et al. 1990) als auch für die Monte(Therburg 1997) und Pampa-Florenprovinz (Cantero 1999, Nebbia \& Zalba 2007) eine halophile Gesellschaft beschrieben, in der Cyclolepis genistoides dominiert. Diese Dominanzbestände werden meist von verschiedenen Prosopis-, Lycium- und Atriplex-Arten begleitet. Wie auch in den untersuchten Probeflächen, sind nach Lewis et al. (1990) Lycium americanum und Maytenus vitis-idaea im argentinischen Chaco die wichtigsten Begleitarten.

Die hier beschriebene Bulnesia sarmientoi-Gouinia paraguayensis-Gesellschaft kann der Waldformation „Palosantal-Labonal“, wie sie von Spichiger et al. (1991), Huespe et al. (1991) und Prado (1993a) beschrieben wird, zugeordnet werden. Laut Prado (1993a) werden die „Palosantales“ von Bulnesia sarmientoi dominiert und häufig von Aspidosperma triternatum, Tabebuia nodosa, Prosopis kuntzei und Salta triflora begleitet. Der „Matorral xerohigrófilo de Bulnesia sarmientoi y Tabebuia nodosa“, wie diese Formation von Spichiger et al. (1991) genannt wird, wächst auf tonig-lehmigen oder tonigen salzhaltigen Böden, die temporär überschwemmt werden. Huespe et al. (1991) beschreiben den „Palosantal-Labonal“ als heterogene Einheit, in der auch in einigen Zonen Salta triflora dominierend oder sogar in Reinbeständen vorkommen kann. 
Die für das Biosphärenreservat im Norden des paraguayischen Chaco von Navarro et al. (2006) beschriebene Prosopis rojasiana-Bulnesia sarmientoi-Gesellschaft weist in ihrer Artenzusammensetzung Ähnlichkeiten mit der Bulnesia sarmientoi-Gouinia paraguayensisGesellschaft (Einheit 17) auf. Die im paraguayischen Chaco endemische Prosopis rojasiana kommt jedoch in der hier beschriebenen Gesellschaft gar nicht und im Untersuchungsgebiet insgesamt nur selten vor.

Die Phyllostylon rhamnoides-Trithrinax schizophylla-Gesellschaft (Einheit 18), kann der Waldformation „Bosque xerofítico denso semicaducifolio“ (Mereles 2005) und “Quebrachal de Quebracho blanco y Samu'u” (Huespe et al. 1991) zugeordnet werden. Die Gesellschaft kann außerdem mit der von Fuentes \& Navarro (2000) für den bolivianischen Chaco beschriebenen Diplokeleba floribunda-Trithrinax schizophylla-Gesellschaft (Diplokelebo floribundae-Trithrinacetum schizophyllae) auf schwach dränierten, tonigen und salzhaltigen Böden verglichen werden. Trotz vieler Ähnlichkeiten in der Artenzusammensetzung, kommen jedoch mehrere Kennarten, wie z.B. Diplokeleba floribunda und Aspidosperma triternatum, im Untersuchungsgebiet kaum vor.

Die in dieser Studie beschriebene Schinopsis cornuta-Elionurus muticus-Gesellschaft bezieht sich auf die Baumsavanne in fossilen mit Sand gefüllten Flusssystemen im Chaco Boreal, die von anderen Autoren auf Formationsebene als „sabana clara arbolada con espartillo“, „campo espartillar“, „espartillar“ (Ramella \& Spichiger 1989, Spichiger et al. 1991, Pérez de Molas 1994, Mereles 2005) und „Vegetation der Sandflächen des Chaco Boreal“ (Esser 1982) bezeichnet worden ist. Die fossilen mit Sand gefüllten Flussbetten, die auch „paleocauces colmatados“ genannt werden, befinden sich meist in einer topografisch höheren Lage. Laut Pérez de Molas (1994) weist die Flora dieser Gesellschaft eine enge Beziehung zu den „campos cerrados“ im Südwesten Brasiliens und im Nordosten Paraguays auf.

Bei Vergleichen mit den Gehölzgesellschaften der argentinischen Salzstandorte fallen sowohl strukturelle als auch Arten- und Dominanzunterschiede auf. So kommen laut Therburg (1997) mehrere Atriplex-Strauchgesellschaften in Salzstandorten der Monte-Florenprovinz vor. Im Untersuchungsgebiet ist die Gattung dagegen nur mit einer Art vertreten, die zudem nur begleitend und in niedriger Frequenz auftritt.

Obwohl der Säulenkaktus Stetsonia coryne häufig in der Gehölzvegetation im Untersuchungsgebiet anzutreffen ist, nimmt er keine gesellschaftsdefinierende Stellung ein. An argentinischen Salzstandorten dagegen bildet diese Art die halophile Gebüschformation „Cardonal de Stetsonia coryne“ (Ragonese 1951, Morello \& Saravia 1959).

In argentinischen halophilen Gehölzgesellschaften fällt zudem die hohe Anzahl von Endemiten, wie z.B. Allenrolfea vaginata, A. patagonica (Amaranthaceae), Ehretia cortesia (Boraginaceae), Grahamia bracteata (Anacampserotaceae) und Plectrocarpa tetracantha (Zygophyllaceae) auf. Das tropisch/subtropisch geprägte Klima und die vergleichsweise hohen Jahresniederschläge im Untersuchungsgebiet dürften diese Gattungen, die nicht in Paraguay vertreten sind, an einer Ausbreitung äquatorwärts hindern. 


\subsubsection{Syntaxonomische Einordnung}

In Anlehnung an Navarro \& Maldonado (2002), Galán de Mera \& Vicente (2006) und Eskuche (1992) kann die Gehölzvegetation der salzbeeinflussten Standorte im Chaco Boreal in zwei Klassen eingeordnet werden.

- Klasse: Ruprechtio triflorae-Schinopsietea lorentzii Rivas-Martínez \& Navarro in Navarro \& Maldonado 2002

Klimax-Wälder in der biogeographischen Chaco-Region. Verbreitet im Süden Boliviens, Nordwesten Paraguays, Norden Argentiniens und extremen Südwesten Brasiliens.

> Ordnung: Ruprechtio triflorae-Schinopsietalia lorentzii Navarro in Navarro \& Maldonado 2002

Klimaxwälder im Chaco Boreal auf gut, mäßig und schwach dränierten Böden.

- Verband: Ruprechtio triflorae-Schinopsion lorentzii Navarro in Navarro \& Maldonado 2002

Xeromorphe Wälder im trockenen und semiariden Chaco Boreal.

+ Phyllostylon rhamnoides-Trithrinax schizophylla-Gesellschaft

$>$ Ordnung: Copernicio albae-Tabebuietalia nodosae Navarro in Navarro \& Maldonado 2002

Edapho-hygrophile Wald-, Gebüsch- und Palmgesellschaften im Chaco auf schwach dränierten bis periodisch überfluteten Böden.

- Verband: Copernicio albae-Tabebuion nodosae Navarro in Navarro \& Maldonado 2002

Wald- und Gebüschgesellschaften auf schwach dränierten und periodisch überfluteten Böden.

+ Parkinsonia aculeata-Copernicia alba-Gesellschaft

+ Prosopis ruscifolia-Sporobolus pyramidatus-Gesellschaft

+ Prosopis ruscifolia-Evolvulus convolvuloides-Gesellschaft

+ Prosopis ruscifolia-Lycium americanum-Gesellschaft

+ Cyclolepis genistoides-Lycium americanum-Gesellschaft

+ Prosopis ruscifolia-Tabebuia nodosa-Gesellschaft

+ Bulnesia sarmientoi-Gouinia paraguayensis-Gesellschaft

\section{- Klasse: Elionuretea mutici Eskuche 1992}

Grasfluren und Baumsavannen mit einer großen Artenvielfalt in der Krautschicht. Die Klasse wurde von Eskuche (1992a, 1992b) für feuchte von Elionurus muticus dominierte Grasfluren und Baumsavannen im Gebiet des Zusammenflusses des Río Paraná und Río Paraguay beschrieben. Aufgrund der vielen gemeinsamen Krautarten werden hier vorläufig eine neue Ordnung und ein neuer Verband für die im Untersuchungsgebiet vorkommende Schinopsis cornuta-Elionurus muticus-Gesellschaft vorgeschlagen.

$>$ Ordnung: Tabebuio aureae-Elionuretalia mutici prov. 
Baumsavannen in fossilen mit Sand gefüllten Flussbetten im paraguayischen Chaco. Kennarten: Tabebuia aurea, Schinopsis cornuta, Astronium fraxinifolium, Jacaranda mimosifolia, Elionurus muticus, Aristida mendocina, Rhynchosia burkartii und Pappophorum krapovickasii

- Verband: Tabebuio aureae-Elionurion mutici prov.

+ Schinopsis cornuta-Elionurus muticus-Gesellschaft

\subsubsection{Strukturelle Vergleiche}

Strukturelle Untersuchungen sind im paraguayischen Chaco bisher häufig auf Formationsebene durchgeführt worden. So zählte Mereles (1998) im feuchten Chaco 396 Individuen mit einem BHD $\geq 10 \mathrm{~cm}$ pro Hektar, von insgesamt 65 Arten, im mesoxerophytischen Schinopsis balansae-Wald. Von Rempel (2007) wurden in einer EinHektar-Daueruntersuchungsfläche in der Waldformation „Palosantal-Labonal“ 431 Individuen mit einem BHD $\geq 10 \mathrm{~cm}$, von insgesamt 21 Arten, gefunden. Die hier präsentierten Ergebnisse lassen sich aufgrund fehlender gesellschaftsspezifischer Untersuchungen im paraguayischen Chaco nur annähernd mit anderen Studien vergleichen.

Hohe Abundanzen sowie hohe Frequenzen, wie bei Prosopis ruscifolia in den Einheiten 11 bis 15 gezählt wurden, sind nach Lamprecht (1986) für horizontal durchgehende Arten kennzeichnend. Bei zusätzlich hoher Dominanz spielen diese Arten die beherrschende Rolle im betreffenden Waldtyp. Auch im argentinischen Chaco wurden laut Rios et al. (2008) in einem „Vinalar“ mit Prosopis ruscifolia 370 Bäume/ha gezählt. In Reinbeständen der Vinalares können laut López et al. (2002) und Rios et al. (2008) insgesamt bis zu 2000 Individuen/ha dieser einen Art auftreten.

Salta triflora erreicht in den untersuchten Waldgesellschaften hohe Abundanzwerte. In Strukturuntersuchungen von Mitlöhner (1990) und Rempel (2007) im Naturreservat Laguna Porá, das direkt neben dem Untersuchungsort Campo María liegt, erreichte auch Salta triflora die höchsten Abundanzwerte. In den Waldgesellschaften befindet sich Salta triflora meist oberhalb der Strauchschicht und kommt deshalb oft in starke Konkurrenz mit Bäumen der unteren Baumschicht, wie Ziziphus mistol, Sideroxylon obtusifolium, Prosopis ruscifolia und Tabebuia nodosa. Laut Jacobs (1990) erhält diese Art dennoch hohe Abundanz durch eine sehr hohe Anzahl an Stockausschlägen. Die höchste individuelle Abundanz wiederum erreicht diese Art laut Mitlöhner (1990) an Standorten mit schlechter Wasserverfügbarkeit.

In der Bulnesia sarmientoi-Gouinia paraguayensis-Gesellschaft (Einheit 17), die der Waldformation „Palosantal-Labonal“ zugeordnet werden kann, weisen Bulnesia sarmientoi, Salta triflora und Ziziphus mistol die höchsten IVI-Werte auf. Die selben Arten, jedoch in umgekehrter Reihenfolge, weisen auch laut Rempel (2007) im „Palosantal-Labonal“ die höchsten IVI-Werte auf.

In Übereinstimmung mit Baumhöhenmessungen im argentinischen trockenen Chaco von Roth \& Giménez (1997), weisen auch im Untersuchungsgebiet die meisten Arten Höhen unter 10 m auf. Nur einige wenige, wie Aspidosperma quebracho-blanco, Astronium fraxinifolium, Bulnesia sarmientoi, Cordia americana, Phyllostylon rhamnoides und Schinopsis cornuta 
erreichen Höhen über 10 m. Im Gegensatz dazu weisen laut Roth \& Giménez (1997, 2006) die meisten Baumarten im feuchten Chaco Höhen über $10 \mathrm{~m}$ auf.

Phyllostylon rhamnoides gehört in der Einheit 18 zur oberen Baumschicht. Im argentinischen feuchten Chaco dagegen kann diese Art laut Hampel (2000) als eine Schatten-Baumart definiert werden, da diese Wälder höher sind und zudem eine dritte Baumschicht aufweisen.

\subsection{4. Ökologische Eigenschaften}

Standorte mit ungünstigen Bedingungen werden laut Vareschi (1980) häufig durch geringe Arten- und hohe Individuenzahlen charakterisiert. Durch eine geringe Anzahl interspezifischer Konkurrenten wird der Standort mit hohen Individuenzahlen weniger Arten ausgefüllt. Dieses trifft insbesondere auf die beschriebenen Prosopis ruscifolia-Gesellschaften zu, wo Prosopis ruscifolia allein oder mit nur wenigen anderen Arten hohe Abundanz-Werte erreicht.

Die meisten Arten der Gattung Prosopis sind an Trockenheit und Bodensalinität angepasst. Prosopis ruscifolia weist nach Antezana et al (2000) die höchste Salztoleranz auf und besiedelt meist tonig-salzhaltige Böden, die periodisch mehrere Monate im Jahr überflutet werden. Laut Meloni et al. (2008) verträgt Prosopis ruscifolia Salzkonzentrationen äquivalent zum Meerwasser, weist osmotische Regulationsmechanismen auf und scheidet toxische Ionen aus den Blättern aus.

An salzbeeinflussten Standorten im Gran Chaco ist Prosopis ruscifolia heimisch. Die Art besiedelt jedoch extrem schnell gestörte Standorte, wie z.B. überweidete Flächen oder anthropogen beeinflusste Palmsavannen, führt so zur schnellen Verbuschung von feuchten Offenland-Flächen und wird daher häufig als Störungszeiger definiert (Morello \& Saravia 1959, Mereles \& Degen 1997). Im Untersuchungsgebiet wurden in halophilen offenen Grasfluren an den Ufern der Lagunen und Salzpfannen viele Sämlinge und Jungpflanzen von Prosopis ruscifolia gefunden. Im Chaco scheint laut Mitlöhner (1990) die Überlebensrate im Keimlingsstadium vom spezifischen Salz- und Bodenwassergehalt abhängig zu sein. Beobachtungen während der Feldarbeiten zufolge sind die Überlebens- und Wachstumsraten dieser Art in niederschlagsarmen Jahren höher als in niederschlagsreichen Perioden und führen daher schneller zur Verbuschung. Überschwemmungen, die länger als sechs Monate andauern, sorgen dagegen für massives Absterben dieser Art.

Cyclolepis genistoides meidet laut Therburg (1997) sehr trockene und feuchte Standorte. Sie vermittelt zwischen den Gesellschaften mit einer guten Wasserversorgung infolge hoher Grundwasserstände oder häufiger Überschwemmungen auf der einen und den auf edaphisch trockeneren Standorten vorkommenden Gesellschaften auf der anderen Seite. Im Untersuchungsgebiet kennzeichnet diese Art auch Standorte mit mittlerer Wasserversorgung und Salinität und vermittelt zusammen mit Lycium americanum und Lophocarpinia aculeatifolia zwischen den Salzmarschen (Kapitel 4) und den Waldgesellschaften auf schwach bis mäßig dränierten Böden.

Die von Schinini (2008) beschriebene Tillandsia mereliana gilt als endemische Art der Salzstandorte im paraguayischen Chaco und ist zudem Kennart der Lycium americanumCyclolepis genistoides-Gesellschaft. Diese Tillandsie wächst wurzellos am Boden im 
Halophytengebüsch und weist eine große Abhängigkeit zur Begleitflora, wie Cyclolepis genistoides und Lophocarpinia aculeatifolia, und den Bodeneigenschaften auf. Das gleichzeitige Auftreten als Epiphyt in einer Höhe von 1 bis 2 m dürfte eine Anpassung an periodische Hochwasser sein. Nach monatelanger Überschwemmung im Untersuchungsgebiet wurden am Boden keine lebenden Individuen gefunden, jedoch konnten zahlreiche epiphytische Individuen beobachtet werden.

In Übereinstimmung mit Untersuchungen in Wäldern im argentinischen feuchten Chaco (Barbaris et al. 2002), hängt auch im Untersuchungsgebiet die Artenzusammensetzung und die damit verbundene Gesellschaft stark von der lokalen Bodenheterogenität ab. Auch Beobachtungen von Mitlöhner (1990) zufolge, ist die Artenzusammensetzung im Chaco schon bei geringsten Niveauunterschieden offensichtlicher Ausdruck hydrischer Variationen. Die Gesellschaften 10 und 11 werden insbesondere von hygrophilen an Stauwasser angepassten Arten charakterisiert. Indikatorarten für schwach dränierte Böden sind laut Navarro \& Maldonado (2002) und Navarro et al. (2011) Prosopis ruscifolia, Tabebuia nodosa, Bulnesia sarmientoi und Trithrinax schizophylla. In der Krautschicht tritt an diesen Standorten sehr häufig Ruellia simplex auf.

Bezüglich der Wasserverfügbarkeit in den Trockengebieten ist die Konkurrenzkraft der Bäume von den standörtlichen Bedingungen und den individuellen Arteigenschaften abhängig (Mitlöhner 1998). Untersuchungen pflanzeninterner Potentiale von Mitlöhner (1990) zeigen, dass Salta triflora, Bulnesia sarmientoi, Sarcotoxicum salicifolium und Bougainvillea campanulata sehr hohe osmotische Spektren aufweisen.

Untersuchungen von Mitlöhner \& Koepp (2007) haben außerdem gezeigt, dass S. triflora extrem hohe osmotische Anpassungsfähigkeit an Bodensalinität aufweist. Aber auch Prosopis ruscifolia und Bulnesia sarmientoi, die mit ihren Wurzeln das salzhaltige Grundwasser erreichen, sind fähig extrem hohe Saugspannungen (>50 bar) zu entwickeln (Glatzle et al. 2000).

Die Gehölzvegetation salzhaltiger Standorte beeinflusst grundlegend die Dynamik des salzigen Grundwassers und spielt eine entscheidende Rolle in der Abschwächung oder Vermeidung oberflächiger Bodenversalzung (Glatzle et al. 2000). Untersuchungen von Glatzle et al. (2006)(2006) während drei Jahren im Untersuchungsgebiet (Naturreservat Campo Maria) haben gezeigt, dass, im Gegensatz zu den Offenland-Standorten der Lagunen und Weideflächen, an Waldstandorten nur geringe Anstiege des Grundwasserspiegels nach Niederschlägen verzeichnet und eine sehr hohe aber nahezu konstante Leitfähigkeit gemessen wurden. 


\section{Abschließende Diskussion und Empfehlungsansätze}

\subsection{Diskussion der wichtigsten Forschungsergebnisse}

Aufgrund der wenigen ökologisch-pflanzensoziologischen Untersuchungen der Vegetation im paraguayischen Chaco, kann die vorliegende Arbeit zu den Pionierarbeiten für die Region und insgesamt für Paraguay angesehen werden. Es ist außerdem die erste Studie dieser Art in den salzbeeinflussten Habitaten im paraguayischen Chaco. In diesem Zusammenhang sollen hier die wichtigsten Ergebnisse hervorgehoben werden.

\subsubsection{Pflanzengeographische Stellung und Artenzusammensetzung der Vegetation salzbeeinflusster Standorte}

Die untersuchten Salzstandorte sind in Übereinstimmung mit Studien anderer Binnenlandsalzstandorte (Ungar 1972, Ragonese 1951, Cantero 1999) artenarm. Von den im paraguayischen Chaco vorkommenden Arten wurden an den untersuchten salzbeeinflussten Standorten 18,9 \% und in den Salzmarschen nur 2,6 \% gefunden.

In ihrer Artenzusammensetzung ist die untersuchte salzbeeinflusste Vegetation größtenteils tropisch/subtropisch geprägt. Pan- und neotropische Elemente nehmen auf Gattungsebene den höchsten Stellenwert ein. Die Vegetation besteht prinzipiell aus im weiteren Sinne neotropischen Arten, wobei die direkte phytogeographische Umgebung des Chaco eine entscheidende Rolle in der Artenzusammensetzung spielt. Entscheidend für die starke Prägung der phytogeographischen Umgebung dürfte die teilweise enge Verbindung der gesamten Chacovegetation mit der Alkalinität, Salinität und Textur des Bodens sein.

In Übereinstimmung mit López et al. (2006), weist der Chaco, und in diesem Zusammenhang auch das Untersuchungsgebiet der Salzstandorte, große floristische Ähnlichkeit mit der südlichen Trockenflora auf und bildet im südamerikanischen Kontext eine gewisse Verbindung zwischen der südlichen und der nördlichen Flora. Ähnlichkeiten zur südlichen Trockenflora werden insbesondere durch die vorkommenden Taxa der Chaco-Florenregion und des Andenvorlandes deutlich.

Die Ergebnisse dieser Studie zeigen deutliche floristische Ähnlichkeiten mit den argentinischen Salzstandorten in der Chaco- (Ragonese 1951, Lewis et al. 1990, Hilgert et al. 2003), Monte- (Therburg 1997) und Pampa-Florenprovinz (Ragonese \& Covas 1947; Cantero 1999). Floristische Ähnlichkeiten, sowohl auf Gattungs- als auch auf Artenebene, gibt es aber auch mit Salzstandorten der Karibik, wie die kolumbianische Halbinsel Guajira (Rieger 1976), die Küstensalzmarschen in Venezuela (Medina et al. 2008) und die kubanischen Küstenregionen (Borhidi et al. 1983, Borhidi 1996, González Gutiérrez et al. 2006).

Salzstandorte in Patagonien (Faggi 1985, Roig et al. 1985) weisen im Gegensatz dazu nur einige wenige gemeinsame Arten mit dem Untersuchungsgebiet auf. Mit den hochandinen Halophytengesellschaften aus Perú (Gutte \& Müller 1985, Montesinos-Tubée 2012), Bolivien (Gehler 1992) und Chile (Teillier \& Becera 2003) konnten auf Artenebene keine Gemeinsamkeiten gefunden werden. 
Im Gegensatz zu den argentinischen Salzstandorten, konnten im Untersuchungsgebiet nur eine Gattung und zehn Arten mit amphitropischer Verbreitung gefunden werden. Mehrere Gattungen argentinischer Halophytengesellschaften wie Frankenia, Suaeda, Allenrolfea, Puccinellia und Distichlis sind im paraguayischen Chaco nicht vertreten. Der Grund hierfür dürfte darin liegen, dass die Trockengebiete Argentiniens das südliche Hauptareal dieser disjunkten Verbreitung darstellen. Das tropisch/subtropisch geprägte Klima und die vergleichsweise hohen Jahresniederschläge im Untersuchungsgebiet dürften vermutlich eine Ausbreitung dieser Gattungen bzw. Arten in den paraguayischen Chaco verhindern.

In dieser Studie wird erstmals das Lebensformenspektrum der vorkommenden Arten im paraguayischen Chaco und in den salzbeeinflussten Habitaten definiert und somit ein überregionaler physiognomischer Vergleich der Vegetation ermöglicht. Binnenlandsalzstellen und Salzwüsten werden laut Chapman (1974), im Gegensatz zu Küstensalzmarschen, hauptsächlich von Sträuchern geprägt. Salzbeeinflusste Standorte im Untersuchungsgebiet und im gesamten Gran Chaco werden ebenfalls von Sträuchern dominiert. Argentinische Binnenlandsalzstandorte in gemäßigten Regionen weisen im Gegensatz dazu eine Dominanz von Kräutern und Kleinsträuchern auf. Es ist daher anzunehmen, dass zunehmende Sonneneinstrahlung und Mitteltemperatur zu einem höheren Anteil von Phanerophyten in salzbeeinflussten Standorten führen. Das Vorherrschen von Phanerophyten, die im Untersuchungsgebiet zum größten Teil zum Chaco-Element gehören, weist auch auf eine klare Verbindung zur jeweiligen phytogeographischen Umgebung hin.

\subsubsection{Pflanzensoziologische, ökologische und strukturelle Eigenschaften der salzbe- einflussten Vegetation}

Die Vegetation salzbeeinflusster Standorte im paraguayischen Chaco besteht zum einen aus Salzmarschen, die ausschließlich aus Kraut- und Zwergstrauchgesellschaften zusammengesetzt sind, und zum anderen aus einer halophilen Gehölzvegetation, die hauptsächlich von Gebüsch- und Waldgesellschaften geprägt wird. In den untersuchten Salzmarschen konnte eine klare Abfolge von neun unterschiedlichen artenarmen Gesellschaften beobachtet werden. Allmähliche Übergänge zwischen den Gesellschaften kommen nur selten vor. In der dominierenden Gehölzvegetation, in der zehn Gesellschaften unterschieden werden konnten, sind die Abfolgen dagegen nicht so eindeutig und allmähliche Übergänge zwischen Gesellschaften sind häufig anzutreffen.

In Übereinstimmung mit Untersuchungen in zentralargentinischen salzbeeinflussten Habitaten (Therburg 1997, Menghi et al. 2001, Karlin et al. 2011) tritt der floristische Hauptgradient im Untersuchungsgebiet von Zonen mit langandauernder Überschwemmung bis zu höher gelegenen Standorten mit mäßig bis gut dränierten Böden auf. Die Salzverhältnisse konnten nur teilweise für die Ausbildung der verschiedenen Halophytengesellschaften verantwortlich gemacht werden. Diskontinuitäten in der Vegetation grundwassernaher Standorte hängen laut Cantero et al. Cantero et al.(1998b) größtenteils von der Tiefe und Salinität des Grundwassers ab, welche wiederum die Bodensalinität kontrollieren. Komplexe Interaktionen zwischen Hydromorphie und Halomorphie bestimmen daher die Verbindungen zwischen salzhaltigen Standorten und den jeweiligen floristischen Gruppen. Die an salzhaltige Böden angepasste Gehölzvegetation im Chaco beeinflusst laut Glatzle et al. (2000) grundlegend die Dynamik 
des salzigen Grundwassers und spielt eine entscheidende Rolle in der Abschwächung oder Vermeidung oberflächiger Bodenversalzung.

Signifikante Bodenparameter in der Verteilung der untersuchten Gesellschaften sind der Natrium-Gehalt und die Bodenart. An den Ufern der Lagunen und Flussbetten wurden zwar nur schwache Reliefunterschiede beobachtet, jedoch beeinflussen diese maßgeblich die Sedimentablagerungen, Wasserverfügbarkeit und Dauer der Überschwemmung. Hygrohalophile Gesellschaften kommen meist in stauwasserbeeinflussten Senken vor, wo hohe Schluff- und Tonanteile im Oberboden zu einer schlechten Dränage führen. Durch Sedimentaufschüttungen entstehen dagegen mikrotopographische Änderungen, die nach Ungar (1972) die Feuchtigkeit und Salinität reduzieren. Der sandige Horizont, der durch Sedimentaufschüttungen entsteht, reduziert laut Karlin et al. (2011) den kapillaren Aufstieg des salzigen Grundwassers und erleichtert so die Etablierung von Arten mit geringerer Salztoleranz.

Mit zunehmender Entfernung von den grundwasserbeeinflussten Salzstandorten kann eine Zunahme an Arten- und Strukturdiversität beobachtet werden. Während die Gesellschaften der Salzmarschen hauptsächlich aus einer Vegetationsschicht und einer Differenzialart bestehen, werden die mehrschichtigen Gehölzgesellschaften von mehreren Differenzialarten bestimmt. Hohe Abundanzen und Frequenzen einzelner Arten können sowohl in den Salzmarsch- als auch in den Gehölzgesellschaften beobachtet werden. In diesem Zusammenhang kommt Prosopis ruscifolia ein besonders hoher Stellenwert in den Gebüschgesellschaften zu, während Salta triflora in den Waldgesellschaften hohe Abundanzund Frequenzwerte erreicht.

In dieser Studie wird zudem eine syntaxonomische Übersicht der vorkommenden Gesellschaften gegeben. Sowohl die Vegetation der Salzmarschen als auch die Gehölzvegetation kann jeweils in drei unterschiedlichen syntaxonomischen Klassen eingeordnet werden.

\subsection{Offene Fragen und zukünftige Forschungsansätze}

Die Ergebnisse dieser Studie lassen nur ansatzweise Schlüsse über die Dynamik und Sukzessionen der Gesellschaften zu. Aufgrund der begrenzten Zeit sind langfristige Daueruntersuchungen nicht möglich gewesen. Auch können ökologische Eigenschaften einzelner Arten nur teilweise verstanden werden.

In zukünftigen Untersuchungen wäre es daher sinnvoll die Sukzession der Gesellschaften in Daueruntersuchungsflächen und in Verbindung mit der Dynamik des Grundwassers zu untersuchen, um das Verhalten der einzelnen Arten besser verstehen zu können. Studien dieser Art könnten höchstwahrscheinlich auch Auskunft darüber geben, welche spezifischen Faktoren für die doch klaren Abfolgen zwischen den Gesellschaften der Salzmarschen verantwortlich sind.

Spezifische pflanzenökologische Studien, die den Einfluss der Bodensalzgehalte auf die Ionengehalte in den Pflanzen untersuchen, könnten zum besseren Verständnis der Physiologie der halophilen Arten im Chaco beitragen. Ähnliche Untersuchungen sind in diesem Zusammenhang im bolivianischen Altiplano (Gehler 1992) und in der argentinischen Provinz Mendoza (Therburg 1997) vorgenommen worden. 
Da es nur sehr wenige pflanzensoziologische Studien im paraguayischen Chaco gibt, sind zukünftige Untersuchungen dieser Art unbedingt notwendig um die Chacovegetation in ihrer Gesamtheit syntaxonomisch zu erfassen.

\subsection{Entwicklungsprognosen der salzbeeinflussten Habitate im paraguayischen Chaco}

\subsubsection{Klimatische Veränderungen und ihre Auswirkung auf die Salzgebiete}

Einer der Hauptgründe für die Versalzungsproblematik im paraguayischen Chaco ist das Überwiegen der Verdunstungsrate gegenüber der Versickerungsrate. Laut Harder et al. (2004) und Grassi et al. (2005) ist die potentielle Evapotranspiration im zentralen Chaco ungefähr doppelt so hoch als die durchschnittlichen Jahresniederschläge.

Laut Wiebe (2003) wird die Versalzungsproblematik im Chaco zudem durch starke klimatische Variabilitäten verschärft. Überdurchschnittliche jährliche Niederschlagsmengen beschleunigen die Versalzung durch Anhebung des Grundwasserspiegels und der Mobilisierung von im Bodenkörper gespeicherten Salzen. Geringere Niederschlagsmengen reduzieren dagegen die Versalzung unter Grundwasser-nahen Bedingungen durch das Absenken des Grundwasserspiegels. Globale Klimaschwankungen (wie das Phänomen El Niño-Südliche Oszillation) begünstigen die lokalen Klimaschwankungen. In einigen Zonen des Untersuchungsgebietes, wie z.B. in der Umgebung von Buena Vista, wurden laut Wiebe (2003) die ersten auffälligen Versalzungserscheinungen im Bereich des Lagunensystems nach einem El-Niño-Ereignis im Sommer 1982/83 beobachtet. In Tabelle 2-5 werden die starken Unterschiede in jährlichen Niederschlagssummen deutlich. In diesem Zusammenhang begünstigen besonders auch einzelne überdurchschnittliche Niederschlagsphänomene, wie z.B. langandauernde Überschwemmungen, die Expansion der Salzpfannen und verhindern die Etablierung von Gehölzen in den Salzsteppen.

Bezüglich der Dynamik in salzbeeinflussten Standorten führen laut Karlin et al. (2011) aufeinanderfolgende Jahre mit überdurchschnittlichen Niederschlägen zu starken Erosionsund Oberflächenabflussprozessen und fördern zudem einen starken Anstieg des Grundwasserspiegels. Besonders gefährdet sind diesbezüglich stark anthropogen beeinflusste Flächen. In aufeinanderfolgenden Jahren mit unterdurchschnittlichen Niederschlägen kommt es im Gegensatz dazu zu Sedimentaufschüttungen und Sandanhäufungen, die die Etablierung von Pioniergesellschaften begünstigen. Sind die Niederschläge jedoch zu niedrig, können sich keine Pflanzengesellschaften etablieren und so werden Desertifikationsprozesse gefördert.

Den klimatischen Prognosen von Bidegain et al. (2012) zufolge, sind im Untersuchungsgebiet im Vergleich zum heutigen Klima zukünftige Anstiege der Mitteltemperatur und Reduzierungen der mittleren Jahresniederschläge zu erwarten. Ohne konkrete Naturschutzmaßnahmen sind in diesem Zusammenhang in Zukunft mit Zunahmen von Versalzungs- und Desertifikationserscheinungen zu rechnen. 


\subsubsection{Auswirkungen des immer stärker werdenden Landnutzungswandels}

Der paraguayische Chaco bekommt immer größere land- und viehwirtschaftliche Bedeutung. In den letzten Jahrzehnten wird der Chaco zunehmend von großen Agrar- und Rinderfarmbetrieben erschlossen und zum Teil intensiv land- und viehwirtschaftlich genutzt. Durch großflächige Rodungen werden die Chacowälder in Kulturland umgewandelt und die Agrarfront breitet sich auch in die trockeneren und versalzungsgefährdeten Gebiete aus. Während in den Jahren 1990 bis 2000 die durchschnittliche Abholzungsrate im paraguayischen Chaco noch bei 105.557 ha/Jahr lag (Mereles \& Rodas 2009), wurde in den Jahren 2010 bis 2012 eine mittlere Abholzungsrate von 262.275 ha/Jahr registriert (Asociación Guyra Paraguay/AVINA 2013).

Die zunehmende Nutzung der versalzungsgefährdeten Zone, vor allem weidewirtschaftlich, führt nach Wiens (1998) zu einer Störung in der Wasserhaushaltsregulierung. Entwaldung und die damit verbundene Änderung der Vegetationsdecke haben in dieser sensiblen Zone einen Anstieg des hochversalzten Grundwasserspiegels mit nachfolgender Oberflächenversalzung zur Folge. Hinzu kommen nicht angepasste Infrastrukturmaßnahmen, die in den letzten Jahrzehnten zu einer Ausweitung von Versalzungserscheinungen geführt haben. Insbesondere durch die Anlage von Wegen und Abdämmung der Flusssysteme wird der natürliche Wasser- und Salztransport verringert.

Das Abdämmen zieht laut Wiebe (2003) zwei wesentliche Reaktionen nach sich. Zum einen kommt es nach Starkniederschlägen zu einem Rückstau mit indirektem Grundwasseranstieg und verminderten Wasser- und Salzabtransport. Des Weiteren wird im Unterlauf das salzhaltige Grundwasser nach dem Prinzip der kommunizierenden Röhren hochgedrückt und durch Verdunstungsprozesse eingedampft.

Die Abdämmung periodisch wasserführender Flusssysteme zeigt aber auch ein Dilemma mit dem Farmer im paraguayischen Chaco, und insbesondere im Untersuchungsgebiet, zu kämpfen haben. Die durch Abdämmung künstlich angelegten Wasserreservoire sind notwendig um in den monatelangen Dürreperioden genügend Wasser für die Rinder zu haben; andererseits führen aber gerade diese Staudämme zu weiteren Versalzungserscheinungen.

Eines der großen Probleme für die Entwicklung des Chaco ist der Mangel an Süßwasser. Das Grundwasser ist zum größten Teil hoch versalzen und die wenigen Süßwasservorkommen sind örtlich begrenzt (Kruck et al. 1998). Da der Zugang zum Süßwasser für die ständig wachsende Bevölkerung im Zentralen Chaco gesichert sein muss, laufen aktuell verschiedene Projekte um das Problem des Trinkwassers zu lösen. In den letzten Jahren sind im zentralen Chaco (Filadelfia und Villa Choferes del Chaco) zwei Entsalzungsanlagen installiert worden, um das hochversalzte Grundwasser zu entsalzen. Hierbei wird das salzhaltige Grundwasser aus einer Tiefe von >120 m mit einem Umkehrosmose-Prozess entsalzen und die restliche Salzlauge anschließend wieder in eine höher gelegene Grundwasserschicht infiltriert. Es bleibt abzuwarten, welche Auswirkungen diese Eingriffe für den Chaco haben werden.

Außerdem wird zurzeit ein 200 km langer Aquädukt vom Rio Paraguay (Puerto Casado) in den Zentralen Chaco (Loma Plata) gebaut. Mit dieser Wasserleitung soll der zentrale Chaco, und insbesondere die Indianersiedlungen, mit Trinkwasser versorgt werden. Die offene Frage bei diesem Projekt bleibt jedoch, welchen Einfluss eine ständige Wasserzufuhr auf das sensible Ökosystem des Chacos haben wird. Es ist anzunehmen, dass dadurch das 
Gleichgewicht des Wasserhaushaltes gestört wird und mehr Versalzungserscheinungen auftreten könnten.

Da die Ökonomie des Chacos hauptsächlich von der landwirtschaftlichen und agrarindustriellen Produktion abhängt, ist das Wasser der entscheidende Faktor für Ernteerträge und nachhaltige Produktion. Erste großflächige Bewässerungsversuche haben laut Harder et al. (2004) zu sehr guten Erträgen geführt. Aufgrund der sehr hohen Evapotranspiration im Vergleich zu den Niederschlägen besteht bei großflächiger Bewässerung jedoch die Gefahr zur fortschreitenden Bodenversalzung. Harder et al. (2004) weisen daher darauf hin, nicht in stark versalzungsgefährdeten Zonen und hauptsächlich auf sandigen Böden Bewässerungssysteme zu verwenden. Außerdem sollte genau auf die Wasserqualität und das angewendete Bewässerungssystem geachtet werden, um eventuelle Bodenversalzung zu vermeiden.

\subsubsection{Alternative Nutzungsmethoden und notwendige Naturschutzmaßnahmen}

Laut Yensen (2008) wird sich höchstwahrscheinlich weltweit die Landwirtschaft im 21. Jahrhundert auf salzbeeinflusste Standorte ausweiten, da die Süßwasserreserven abnehmen und die Bevölkerung der betroffenen Gebiete gezwungen sein wird, die weit verbreiteten Salzböden und Aquifere zu nutzen. Auch im paraguayischen Chaco breitet sich die Agrarfront in die trockeneren und versalzungsgefährdeten Gebiete aus. Aus diesem Grund wäre es ratsam, insbesondere an den sensiblen Salzstandorten, eine nachhaltige Nutzung der Ressourcen und aktiven Naturschutz zu fördern.

Anstelle einer Landschaftsveränderung und die damit verbundene Zunahme von Versalzungserscheinungen, wäre es ratsam, die vor Ort angepasste Vegetation nicht zu enfernen, sondern nachhaltig zu nutzen. Die in dieser Studie beschriebenen Gehölzgesellschaften sind für die Wasserhaushaltsregulierung und die Vermeidung von Erosion und Oberflächenversalzung von großer ökologischer Bedeutung. Aus dem Grund sollten die gesetzlich vorgeschriebenen Schutzwaldstreifen an den Lagunen, Einschwemmsenken und periodisch wasserführenden Flusssystemen auf jeden Fall intakt gelassen werden, um Ausbreitungen von Versalzungserscheinungen zu verhindern.

In diesem Zusammenhang weisen auch Glatzle et al. (2000, 2006) auf die bedeutende Rolle des Chacowaldes in der Vermeidung der Bodenversalzung hin und schlagen daher für die versalzungsgefährdete Zone ein silvopastorales Weidesystem vor. Neben der Regulierung des hoch anstehendem Grundwasserspiegels und die damit verbundene Verringerung der Oberflächenversalzung, können laut Kaur et al. (2002) bei silvopastoralen Systemen die Fertilität und mikrobielle Aktivität von Salz-Alkaliböden erheblich verbessert werden. Fortschreitende Oberflächenversalzung könnte auf offenen Weideflächen in der Nähe der Salzpfannen höchstwahrscheinlich durch silvopastorale Systeme oder Wiederaufforstungen vorgebeugt werden. Wiederaufforstungsmaßnahmen könnten laut Wiebe (2003) den Spiegel des salzhaltigen Grundwassers relativ zum Grundwasserstand unter Weide absenken. Laut Taleisnik \& López (2011) wurden bei Aufforstungsversuchen in argentinischen Salzgebieten hauptsächlich heimische Arten der Gattung Prosopis verwendet.

Die Vegetation der untersuchten salzbeeinflussten Standorte weist ein großes Potential für eine nachhaltige Nutzung der Resourcen auf. Hingewiesen sei an dieser Stelle auf einige 
interessante Nutzpflanzen, wie z.B. Bulnesia sarmientoi („Palo santo“) oder Cyclolepis genistoides („Palo azul“). Beide Arten sind aufgrund der massiven Veränderung und Zerstörung der natürlichen Standorte sowie durch unkontrollierte Nutzung gefährdet.

Eine im Chaco bisher unbekannte Bedeutung könnte jedoch in Zukunft auch dem Queller (Sarcocornia ambigua) zukommen. Untersuchungen in Brasilien von D'oca et al. (2012) haben gezeigt, dass das Samenöl von Sarcocornia ambigua einen sehr hohen Anteil (24.8\%) von gesättigten Palmitin- und Stearinsäuren enthält. Da keine schädlichen FettsäureKomponenten gefunden wurden, könnte das Öl sowohl für die Tierernährung als auch für die Biotreibstoff-Produktion empfohlen werden.

Die untersuchten Salzstandorte bieten ein großes Potential für Ökotourismus- und Umweltbildungsaktivitäten. Die Naturreservate Campo Maria und Chaco Lodge gehören heute zu den bekanntesten Reisezielen. Aufgrund des wachsenden Umweltbewusstseins in der Landesbevölkerung dürften Aktivitäten dieser Art in Zukunft zunehmen. Parallel sind jedoch auch nationale Naturschutzmaßnahmen notwendig, da die bisherigen Naturschutzbemühungen im Untersuchungsgebiet hauptsächlich auf Privatinitiativen zurückgehen.

In Übereinstimmung mit Wiebe (2003) sind insbesondere die Aufklärung der Landnutzer in der versalzungsgefährdeten Zone sowie die Schaffung eines institutionellen Rahmens für Nutzungsauflagen, verbunden mit einer Kontrolle der Einhaltung, unbedingt notwendig um weitere Versalzungserscheinungen vorzubeugen und die sensiblen Salzstandorte zu schützen. 


\section{Zusammenfassungen}

\subsection{Zusammenfassung}

Die vorliegende Arbeit befasst sich mit der Pflanzengeographie, Pflanzensoziologie und Ökologie der salzbeeinflussten Vegetation im Chaco Boreal von Paraguay. Im Mittelpunkt der Betrachtungen stehen die floristischen, strukturellen und ökologischen Merkmale der Vegetation entlang eines Gradienten von stark salzhaltigen bis zu mäßig salzbeeinflussten Standorten und die daraus resultierende vegetationsökologische Charakterisierung.

Das Untersuchungsgebiet liegt in der Übergangszone von den regelmäßig überschwemmten Flächen des feuchten Ostchaco zum trockenen Zentralen Chaco, in der als Folge der klimatischen und geologischen Verhältnisse großflächige Salzstandorte vorkommen. Versalzungen, die in dieser Zone auf natürliche Weise entstehen, treten hier im Bereich von Niederungen und Unterläufen periodisch wasserführender Flusssysteme auf und stehen in enger Verbindung zum hochanstehenden salzigen Grundwasser.

An insgesamt 14 Untersuchungsorten wurden 162 stratifiziert-randomisierte Vegetationsaufnahmen in den Salzmarschen und 236 Aufnahmen in der angrenzenden salzbeeinflusten Gehölzvegetation durchgeführt. Den Bestandsaufnahmen in physiognomisch unterscheidbarer homogener Vegetation folgten Untersuchungen einiger Standortfaktoren.

Die untersuchten Salzstandorte sind in Übereinstimmung mit Studien anderer Binnenlandsalzstellen in Südamerika artenarm. Von den im paraguayischen Chaco vorkommenden Arten wurden an den untersuchten salzbeeinflussten Standorten 18,9 \% und in den Salzmarschen nur 2,6 \% gefunden.

Pflanzengeographisch wird die untersuchte salzbeeinflusste Vegetation von pantropischen und neotropischen Gattungen dominiert. Im weiteren Sinne neotropische Arten sind vorherrschend, wobei die direkte phytogeographische Umgebung des Chaco eine entscheidende Rolle in der Artenzusammensetzung spielt.

Das Lebensformenspektrum und die lokale Verteilung entlang eines Niederschlagsgradienten der vorkommenden Arten im Untersuchungsgebiet wurde mit der vorab vom Verfasser angefertigten Checkliste der Gefäßpflanzen im paraguayischen Chaco verglichen. Sowohl im paraguayischen Chaco insgesamt als auch an den untersuchten salzbeeinflussten Standorten dominieren Phanerophyten, während in den Salzmarschen Hemikryptophyten vorherrschen.

In den Salzmarschen wurde anhand originaler Vegetationsaufnahmen eine Abfolge von neun Pflanzengesellschaften unterschieden. Die in den meisten Fällen aus nur einer Differenzialart bestehenden Gesellschaften weisen nur selten allmähliche Übergänge auf und kommen entlang eines Gradienten unterschiedlicher Wasserverfügbarkeit vor. Der Gradient reicht von der hydrohalophilen Ruppia maritima-Gesellschaft und der hygrophilen Setaria geminataGesellschaft schwach salziger Standorte bis zur stark salz- und trockenresistenten Heterostachys ritteriana-Gesellschaft.

Als signifikante Bodeneigenschaften, die die Verteilung der Arten in den Salzmarschen bestimmen, konnten der Natriumgehalt und die organische Substanz im Oberboden festgestellt werden. Setaria geminata kommt in Böden mit höherem Humusanteil vor, während in natriumreichen Böden Heterostachys ritteriana und Sarcocornia ambigua 
vorherrschen. Eine bedeutende Rolle spielen außerdem kleine Reliefunterschiede, die maßgeblich die Sedimentablagerungen, Wasserverfügbarkeit und Dauer der Überschwemmung beeinflussen. Sandige Sedimentaufschüttungen reduzieren die Feuchtigkeit und Salinität und erleichtern die Etablierung von Pionierarten, wie Sesuvium portulacastrum und Sporobolus pyramidatus.

Die untersuchte salzbeeinflusste Gehölzvegetation konnte anhand originaler Vegetationsaufnahmen in zehn Gesellschaften klassifiziert werden, die entlang eines Wasserverfügbarkeitsgradienten vorkommen. Entlang dieses Gradienten nimmt die Artenund Strukturdiversität zu und der Anteil an halophilen Arten ab.

Die Bodenparameter, die signifikant die Artenzusammensetzung in den Gebüsch- und Waldgesellschaften beeinflussen, sind der Natriumgehalt und die Bodenart. Hohe Natriumund pH-Werte im Oberboden sind in den untersuchen Probeflächen positiv korreliert und kennzeichnen salzreiche Standorte, die größtenteils von halophilen Arten besiedelt werden. Typische halophile Straucharten sind Cyclolepis genistoides, Lophocarpinia aculeatifolia, Lycium americanum, Lycium boerhaaviaefolium und Prosopis rojasiana.

Die Bodenart im Oberboden spielt für die Artenzusammensetzung eine entscheidende Rolle, da hohe Schluff- und Tonanteile im Oberboden zu einer schlechten Dränage und somit zu stauwasserbeeinflussten Habitaten führen. Dieser Effekt kann ganz besonders an Standorten der Parkinsonia aculeata-Copernicia alba-Gesellschaft, der Prosopis ruscifolia-Sporobolus pyramidatus-Gesellschaft, der Prosopis ruscifolia-Evolvulus convolvuloides-Gesellschaft und der Prosopis ruscifolia-Tabebuia nodosa-Gesellschaft beobachtet werden.

Anhand von Strukturmessungen aller Stämme mit einem BHD $\geq 10 \mathrm{~cm}$ wurden die Abundanz, Frequenz, Dominanz und der Bedeutungswert-Index (IVI) für alle Gehölzarten in den einzelnen Gesellschaften berechnet. Prosopis ruscifolia erreicht hohe Abundanz- und Frequenzwert in den Gebüschgesellschaften, während Salta triflora ein besonderer Stellenwert in den Waldgesellschaften zukommt.

In dieser Arbeit werden die Untersuchungsergebnisse der salzbeeinflussten Vegetation im paraguayischen Chaco mit ähnlichen Studien aus dem südamerikanischen Kontext verglichen und syntaxonomisch eingeordnet. Sowohl die Vegetation der Salzmarschen als auch die Gehölzvegetation kann jeweils in drei unterschiedlichen syntaxonomischen Klassen eingeordnet werden.

Abschließend werden die Auswirkungen und Entwicklungsprognosen hinsichtlich klimatischer Veränderungen und des immer stärker werdenden Landnutzungswandels diskutiert und Ansätze für zukünftige Nutzungs- und Naturschutzmaßnahmen sowie für weitere Untersuchungen empfohlen. 


\subsection{Summary}

The South American Gran Chaco with its particular geological and climatic conditions is especially susceptible to salinization of soils and water. The aim of this study was to analyze the phytogeography and the phytosociology of the salt-affected vegetation in the Paraguayan Chaco and to find patterns of species composition and community structure in relation to different ecological and soil properties.

The study area is located in the transition zone between the regularly inundated humid eastern Chaco and the dry Central Chaco. In these areas widespread natural salinization occurs in lagoons, depressions and along periodical water courses. These salinizations are connected with a shallow saline ground water table.

In total 162 original phytosociological relevés in salt marshes and 236 relevés in salt-affected woody vegetation were collected in 2010-2012 in 14 different study sites. Inventories were associated with the ecological characterization of the habitats.

Similar to other inland salt areas in South America, the examined salt habitats present a low species diversity. From the total species diversity in the Paraguayan Chaco, $18.9 \%$ of the species were found in the whole salt-influenced study area and only $2.6 \%$ in the salt marshes. The examined salt-affected vegetation is dominated by pantropical and neotropical genera. At species level neotropical elements in the broader sense are prevailing, in which the typical Chacoan species play a crucial role in the species composition.

For the analysis of the life form composition and the distribution along a precipitation gradient the species of the study area have been compared with the checklist of the vascular plants of the Paraguayan Chaco that was prepared and recently published by the author. The Paraguayan Chaco as well as the investigated salt-affected habitats are dominated by phanerophytes, whereas in the salt marshes hemicryptophytes are prevailing.

In the salt marshes nine plant communities were identified along a salinity gradient. The plant communities are mostly dominated by only one plant species depending on the degree of soil salinisation and water supply. The communities range from the hydro-halophytic Ruppia maritima community and the hygrophytic Setaria geminata in low saline conditions to the drought and salt resistant Heterostachys ritteriana community.

The species distribution in the salt marshes is significantly influenced by sodium content and the organic matter in the topsoil. Setaria geminata grows in humid soils with a higher humus content, whereas Heterostachys ritteriana and Sarcocornia ambigua grow in soils rich in sodium. Minor topographic differences influence the sediment deposition, water supply and duration of inundation considerably. Sandy sediment accumulations reduce the humidity and salinity and facilitate pioneer species such as Sesuvium portulacastrum and Sporobolus pyramidatus.

The salt-affected woody vegetation has been classified into ten communities along a gradient of decreasing water supply and salinity. Along this gradient the species diversity and structural diversity increased and the proportion of halophyte abundance declines.

The species composition in the shrub and forest communities is greatly influenced by the sodium, sand and silt content in the topsoil. High sodium content and $\mathrm{pH}$ in the topsoil are positively correlated and characterize salt habitats with halophilous shrubs such as Cyclolepis 
genistoides, Lophocarpinia aculeatifolia, Lycium americanum, Lycium boerhaaviaefolium and Prosopis rojasiana.

High silt and clay content in the topsoil determine the drainage capacity of the soils and with it the species composition. Characteristic plant communities on poorly drained soils are the Parkinsonia aculeata-Copernicia alba community, Prosopis ruscifolia-Sporobolus pyramidatus community, Prosopis ruscifolia-Evolvulus convolvuloides community and the Prosopis ruscifolia-Tabebuia nodosa community

The abundance, frequency, dominance and the importance value index (IVI) were calculated for each woody species with a $\mathrm{DBH} \geq 10 \mathrm{~cm}$ in each community. In shrub communites Prosopis ruscifolia occurs with high abundance and frequency, while in forest communities Salta triflora is the most abundant and frequent species.

Furthermore, the salt-affected plant communities of the Paraguayan Chaco are compared with those found in related studies in the South American context. A syntaxonomical scheme for the plant communities is also presented. The vegetation of the salt marshes as well as the woody vegetation can be classified each into three different syntaxa at class level.

Finally, effects of global change and developments of the salt areas in the Paraguayan Chaco in consequence of climatic variation and increasing land use are discussed. Recommendations for future use and conservation measures as well as further research activities are also suggested. 


\subsection{Resumen}

El presente trabajo de investigación analiza la fitogeografía, fitosociología y ecología de la vegetación de los saladares en el Chaco Boreal, Paraguay. El estudio hace hincapié en las características florísticas, ecológicas y estructurales de la vegetación a lo largo del gradiente salino desde zonas bajas con suelos fuertemente salinos hasta zonas con un relieve elevado y una influencia salina moderada.

El área de estudio se encuentra ubicado en la franja de salinización que aparece en la zona de transición entre el Chaco húmedo y el Chaco central seco. Debido a las características geológicas y climáticas se desarrollan en esta zona fenómenos de salinzación en lagunas, riachos y cauces. Estos fenómenos de salinización son en gran parte naturales y se encuentran ligados a la napa freática salada y elevada en la zona.

Los relevamientos fitosociológicos se realizaron en 14 lugares de muestreo durante los meses de verano de 2010-2012. Con un muestreo aleatorio estratificado fueron realizados 162 inventarios en las estepas salinas y 236 inventarios en la vegetación leñosa adyacente. Los inventarios fitosociológicos en vegetación fisionómicamente homogénea fueron acompañados de caracterizaciones ecológicas del hábitat.

En coincidencia con inventarios florísticos en otros saladares continentales de Sudamérica, el área de estudio se caracteriza por una diversidad baja de especies. De las especies vegetales presentes en el Chaco paraguayo, fueron encontrados 18,9 \% en el área de estudio y sólo 2,6 $\%$ en las estepas salinas.

En la vegetación analizada dominan los géneros pantrópicos y neotrópicos. A nivel de especies se puede observar una marcada predominancia de especies con distribución neotrópica en sentido amplio. Las especies típicas de la provincia fitogeográfica chaqueña juegan en este sentido un rol especial en la composición florística.

El espectro de las formas de vida y la distribución geográfica a lo largo del gradiente de precipitación de las especies en el área de estudio fueron comparados con la checklist de la flora vascular del Chaco paraguayo preparada y publicada recientemente por el autor. En el Chaco paraguayo y también en el área de estudio predominan las fanerófitas, mientras que las estepas salinas se encuentran dominadas por las hemicriptófitas.

En las estepas salinas fueron clasificadas nueve comunidades vegetales con secuencias bien definidas y generalmente sin transiciones graduales entre ellas. La mayoría de las comunidades se caracteriza por una sola especie diferencial respectivamente. Las comunidades diferenciadas se encuentran ubicadas a lo largo de un gradiente de disponibilidad de agua, desde la comunidad hidrohalófila de Ruppia maritima y la comunidad higrófila de Setaria geminata en suelos húmedos y salobres hasta la comunidad xerohalófila de Heterostachys ritteriana.

El contenido de sodio y la materia orgánica en los horizontes superiores del suelo tienen una influencia significativa en la distribución de las especies en las estepas salinas. Setaria geminata se desarrolla en suelos húmedos con cierto porcentaje de materia orgánica y muy bajo contenido de sodio. En los suelos con alto contenido de sodio predominan generalmente Heterostachys ritteriana y Sarcocornia ambigua. Pequeñas diferencias del relieve juegan además un importante rol en la distribución de las especies porque influyen en forma decisiva las sedimentaciones, la disponibilidad de agua y la duración de la inundación. Acumulaciones 
de sedimentos arenosos reducen la humedad y la salinidad y facilitan el crecimiento de especies pioneras como Sesuvium portulacastrum y Sporobolus pyramidatus.

Las diez comunidades vegetales diferenciadas en la vegetación leñosa de los saladares del Chaco paraguayo se encuentran ubicados a lo largo de un gradiente de humedad y salinidad. A lo largo de este gradiente aumenta la diversidad estructural y de las especies y disminuye el procentaje de especies halófilas.

El contenido de sodio y el porcentaje de arena y limo en el suelo tienen una influencia significativa en la distribución y composición de las especies en las comunidades arbustivas y boscosas. Altos contenidos de sodio y pH están positivamente correlacionados y caracterizan suelos salinos. En estos hábitats se desarrollan principalmente especies halófilas, entre ellas varios arbustos como Cyclolepis genistoides, Lophocarpinia aculeatifolia, Lycium americanum, Lycium boerhaaviaefolium y Prosopis rojasiana.

Altos porcentajes de limo y arcilla en el suelo caracterizan suelos mal drenados e influyen significativamente la composición de las especies en una comunidad determinada. Este efecto se pudo observar especialmente en las comunidades de Parkinsonia aculeata-Copernicia alba, Prosopis ruscifolia-Sporobolus pyramidatus, Prosopis ruscifolia-Evolvulus convolvuloides y Prosopis ruscifolia-Tabebuia nodosa.

Mediante mediciones estructurales de los troncos con un DAP $\geq 10 \mathrm{~cm}$ fueron calculados la abundancia, frecuencia, dominancia y el índice de valor de importancia (IVI) para las especies leñosas presentes en cada comunidad. En las comunidades arbustivas la especie más abundante y frecuente es Prosopis ruscifolia, mientras que la especie con mayor abundancia y frecuencia en las comunidades boscosas es Salta triflora.

En el presente trabajo se comparan las comunidades vegetales diferenciadas en los saladares del Chaco paraguayo con otros estudios similares en el contexto sudamericano y se presenta una esquema sintaxonómico de las mismas. Las comunidades vegetales de las estepas salinas y tambien de la vegetación leñosa pueden ser clasificadas en tres clases sintaxonómicas respectivamente.

Teniendo en cuenta las alteraciones climáticas y el constante aumento del uso de la tierra en el Chaco paraguayo se realiza al final del trabajo una discusión acerca de las consecuencias y posibles pronósticos de desarrollo en los saladares. Además se presentan algunas recomendaciones para futuras investigaciones, usos alternativos y medidas necesarias para la conservación de los saladares estudiados. 


\section{Abkürzungsverzeichnis und Glossar}

\subsection{Abkürzungen}

\begin{tabular}{|c|c|}
\hline Abb. & Abbildung \\
\hline AMER & Amerikanisch \\
\hline AMPHI & Amphitropisch \\
\hline BHD & Brusthöhendurchmesser \\
\hline $\mathrm{Ca}$ & Calcium \\
\hline $\mathrm{CaCO} 3$ & Calciumcarbonat \\
\hline CCA & Kanonische Korrespondenzanalyse (Canonical Correspondence Analysis) \\
\hline Cen & Centinela \\
\hline Ch frut & holzige Zwergsträucher \\
\hline Ch herb & Krautige Chamaephyten \\
\hline Ch succ & Niedrigwüchsige Sukkulenten \\
\hline Ch suff & Halbholzige Zwergsträucher \\
\hline CHA & Gran Chaco \\
\hline CL & Ramsargebiet Chaco Lodge \\
\hline $\mathrm{CM}$ & Naturschutzreservat Campo María \\
\hline DCA & Korrespondenzanalyse (Detrended Correspondence Analysis) \\
\hline DISGEN & Verbreitung der Gattungen \\
\hline DISNEO & Disjunkt Neotropisch \\
\hline DISSPEC & Verbreitung der Arten \\
\hline DOMCHA & Florenregion Chaco \\
\hline DOMNSTW & Florenregion Neotropische Saisontrockenwälder \\
\hline Dpto. & Departamento \\
\hline E herb & Krautige Epiphyten \\
\hline E succ & Sukkulente Epiphyten \\
\hline EL/ Elek. Leit. & Elektrische Leitfähigkeit \\
\hline End & Endemisch im paraguayischen Chaco \\
\hline EndCS & Endemisch im Conosur von Südamerika \\
\hline EndGC & Endemisch im Gran Chaco \\
\hline FaCEN & Facultad de Ciencias Exactas y Naturales \\
\hline Fam. & Familie \\
\hline FCQ & Facultad de Ciencias Químicas \\
\hline FS & Reserva Natural Fortín Salazar \\
\hline G bulb & Knollengeophyten \\
\hline G rad & Wurzelgeophyten \\
\hline G rhiz & Rhizomgeophyten \\
\hline Gen. & Gattung (Género) \\
\hline H caesp & horstige Hemikryptophyten \\
\hline H rept & niederliegende Hemikryptophyten \\
\hline $\mathrm{H}$ ros & rosettenförmige Hemikryptophyten \\
\hline H scap & aufrechte Hemikryptophyten \\
\hline HPar & Hemiparasiten \\
\hline Hyd rad & Wurzelhydrophyten \\
\hline Hyd rhiz & Rhizomhydrophyten \\
\hline IVI & Bedeutungswert-Index (Importance Value Index) \\
\hline KOS & Kosmopolitisch \\
\hline
\end{tabular}




\begin{tabular}{|c|c|}
\hline $\mathrm{L}$ frut & holzige Lianen \\
\hline L herb & krautige Lianen \\
\hline L suff & halb-holzige Lianen \\
\hline LC & Laguna Capitán \\
\hline LE & Campo León \\
\hline LF & Lebensformen \\
\hline LG & Laguna Ganso \\
\hline $\mathrm{Mg}$ & Magnesium \\
\hline $\mathrm{Na}$ & Natrium \\
\hline Nat & Heimisch (Nativo) \\
\hline Natur & eingebürgerte Art (especie naturalizada) \\
\hline NEO & Neotropisch \\
\hline NG & Niederschlagsgradient \\
\hline Org & Organische Substanz \\
\hline P caesp & Sträucher \\
\hline P dol & Flaschenbäume \\
\hline P gram & graminoide Phanerophyten \\
\hline P herb & Hochstauden \\
\hline P ros & rosettenartige Phanerophyten (Palmen) \\
\hline P scap & Bäume \\
\hline $\mathrm{P}$ succ & hohe Sukkulenten \\
\hline PAL & Paläotropisch \\
\hline PAN & Pantropisch \\
\hline Par & Parasiten/ Holoparasiten \\
\hline PCA & Hauptkomponentenanalyse (Principal Component Analysis) \\
\hline PY & Paraguay \\
\hline RF & Reserva Natural Riacho Florida \\
\hline RG & Riacho González \\
\hline $\mathrm{RM}$ & Riacho Mosquito \\
\hline RS & Ramsargebiet Laguna Rojas Silva \\
\hline RV & Río Verde \\
\hline sp. & Art (species) \\
\hline subsp. & Unterart (subspecies) \\
\hline SURAMA & Florenregionen südlich des Amazonas \\
\hline T caesp & verzweigte oder horstige Terophyten \\
\hline T rept & niederliegende Terophyten \\
\hline T scap & aufrechte Terophyten \\
\hline T succ & Sukkulente Terophyten \\
\hline Tab. & Tabelle \\
\hline $\mathrm{TL}$ & rankende Terophyten \\
\hline U.N.A. & Universidad Nacional de Asunción \\
\hline var. & Varietät \\
\hline VORAND & Meridionales Andenvorland \\
\hline YP & Reserva Natural Yaguareté Porã \\
\hline
\end{tabular}




\subsection{Glossar}

BedeutungswertIndex

Biosphärenreservat des paraguayischen Chaco

Cardonal

Chaco Boreal

Conosur

Espartillar

Gran Chaco

Labonal

Matorral

Monte

Palosantal

Pampa
Der Bedeutungswert-Index oder Importance-Value-Index wird durch die Summierung von relativer Abundanz, relativer Frequenz und relativer Dominanz für jede Art berechnet und ermöglicht einen annähernden Vergleich ihrer ökologischen Gewichte im betreffenden Waldtyp (Lamprecht 1986).

Von der UNESCO anerkanntes 4.707.250 ha großes Reservat im nördlichen Teil des paraguayischen Chaco. Kernzonen des Biosphärenreservats sind: Parque Nacional Rio Negro, Parque Nacional Defensores del Chaco, Parque Nacional Médanos del Chaco, Parque Nacional Teniente Agripino Enciso, das Reservat für den zukünftigen Parque Nacional Cerro Cabrera - Timane und das Monumento Natural Cerro Chovoreca.

Gebüschformation, in der der Säulenkaktus "Cardón" (Stetsonia coryne) dominiert. Der Begriff wird hauptsächlich in Argentinien verwendet.

Nördlicher Teil des Gran Chaco oder auch der paraguayische und bolivianische Chaco.

Südkegel Südamerikas bestehend aus Argentinien, Chile, Uruguay, Paraguay und den Südosten Brasiliens.

Savanne oder Baumsavanne, in der Gräser des Espartillo (Elionurus muticus) dominieren. Der Begriff wird aber auch für Graslandschaften mit anderen ähnlich aussehenden Grasarten verwendet.

Florenprovinz mit Trockenwäldern und Dornbuschsavannen im Zentrum Südamerikas. Nach Cabrera \& Willink (1973) der ChacoFlorenregion zugeordnet. Der Name "Chaco" stammt aus der indigenen Sprache Quechua und bedeutet "Jagdgebiet".

Hygrophile Waldformation, in der Labón-Bäume (Tabebuia nodosa) dominieren.

Gebüsch oder Gestrüpp. Der Begriff wird häufig in Verbindung mit gebüschartigen Vegetationsformationen verwendet.

Florenprovinz mit trockenem Buschland in Argentinien. Nach Cabrera \& Willink (1973) der Chaco-Florenregion zugeordnet.

Xeromorphe Waldformation, in der Palo santo-Bäume (Bulnesia sarmientoi) dominieren.

Florenprovinz mit Grassteppen im südöstlichen Südamerika. Nach Cabrera \& Willink der Chaco-Florenregion zugeordnet. 
Pantanal

Quebrachal

Reserva Natural

Riacho

Sabana arbolada

Saladar

Salzmarsch

Vinalar

xeromesophytisch
Binnenland-Feuchtgebiet im Südwesten Brasilien. Kleine Teile des Pantanals reichen bis in den Osten Boliviens und den Nordosten des paraguayischen Chaco

Die Quebracho-Bäume (Arten der Gattungen Aspidosperma und Schinopsis) sind durch ihr hartes Holz bekannt. Der Begriff "Quebracho" stammt aus der spanischen Sprache "quiebra hacha" und bedeutet "der die Axt bricht". Quebrachales sind Waldformationen, in den die Quebracho-Bäume dominieren oder entscheident die Waldstruktur beeinflussen.

Naturschutzreservat. Im paraguayischen Naturschutz-System wird der Status „Reserva Natural“ exklusiv für private unter Schutz gestellte Gebiete verwendet. Diese Reservas Naturales werden staatlich anerkannt und können auf bestimmte oder unbestimmte Zeit als solche ausgewiesen werden.

Periodisch wasserführendes Flusssystem.

Baumsavanne

in Paraguay gebräuchliche Bezeichnung für einen Salzstandort. In den Nachbarländern werden auch die Begriffe "Salar", "Salitral" oder "Salina" verwendet.

Offenland-Salzvegetation, in der Kleinsträucher und Kräuter dominieren.

Gebüsch mit Viñal (Prosopis ruscifolia). Der Begriff bezieht sich hauptsächlich auf die Vegetationsformation, in der Prosopis ruscifolia vorherrschend ist.

Zwichen xerophytisch und mesophytisch. Der Begriff beschreibt einen Übergangscharakter. Manche Autoren verwenden auch den Begriff mesoxerophytisch. 


\section{Literaturverzeichnis}

Abrol, I., Yadav, J. \& Massoud, F.I. (1988). Salt-affected soils and their management. Rome.

Albert, R. (1982). Halophyten. In: Kinzel, H. (Hrsg.). Pflanzenökologie und Mineralstoffwechsel. Stuttgart: E. Ulmer. S. 33-213.

Alonso, M.A. \& Crespo, M.B. (2008). Taxonomic and nomenclatural notes on South American taxa of Sarcocornia (Chenopodiaceae). Ann. Bot. Fennici 45: 241-254.

Angiosperm Phylogeny Group (2009). An update of the Angiosperm Phylogeny Group classification for the orders and families of flowering plants: APG III. Bot. J. Linn. Soc. 161: 105-121.

Antezana, C., Atahuachi, M., Arrázola, S., Fernández, E. \& Navarro, G. (2000). Ecología y Biogeografía del Género Prosopis (Mimosaceae) en Bolivia. Rev. Bol. Ecol. 8: 25-36.

Asociación Guyra Paraguay/AVINA (2013). Resultados del Monitoreo de los cambios de uso de tierra, incendios e inundaciones del Gran Chaco Americano. Informe Final - Año 2012. (Informe técnico).

Austin, D.F. (2000). A revision of Cressa L. (Convolvulaceae). Bot. J. Linn. Soc. 133: 27-39.

Barbaris, I.M., Batista, W.B., Pire, E.F., Lewis, J.P. \& León, R. (2002). Woody population distribution and environmental heterogeneity in a Chaco forest, Argentina. J. Veg. Sci. 13: 607-614.

Barros, V.R., Doyle, M.E. \& Camilloni, I.A. (2008). Precipitation trends in southeastern South America: relationship with ENSO phases and with low-level circulation. Theor. Appl. Climatol. 93: 19-33.

Bender, F. (1961). Ergebnisse hydrogeologischer Untersuchungen im Chaco Boreal von Paraguay. Geol. Jb. 78: 693-718.

Bernardi, L. (1984). Contribución a la dendrología paraguaya I. Boissiera 35: 1-341.

Bernardi, L. (1985). Contribución a la dendrología paraguaya II. Boissiera 37: 7-294.

Bidegain, M., Coronel, G., Ríos, N. \& de los Santos, B (2012). Escenarios Climáticos futuros para Paraguay. Meteorologica, 37(2): 47-55.

Borhidi, A. (1996). Phytogeography and Vegetation Ecology of Cuba. Budapest: Akadémiai Kiadó.

Borhidi, A., Muñiz, O. \& Del Risco, E. (1983). Plant communities of Cuba I. Fresh and salt water, swamp and coastal vegetation. Acta Bot. Hung. 29(1-4): 337-376.

Bouyoucos, G.J. (1962). Hydrometer method improved for making particle size analysis of soils. Agron. J. 54: 464-465.

Braun-Blanquet, J. (1964). Pflanzensoziologie - Grundzüge der Vegetationskunde. Berlin/Wien/New York: Springer.

Breckle, S.W. (1976). Zur Ökologie und zu den Mineralstoffverhältnissen absalzender und nicht absalzender Xerohalophyten. Unter besonderer Berücksichtigung von Untersuchungen an Atriplex confertifolia und Ceratoides lanata in Utah/USA. Vaduz: Cramer.

Burkart, A. (1969). Flora ilustrada de Entre Ríos (Argentina). Parte II. Gramíneas. Buenos Aires: Instituto Nacional de Tecnología Agropecuaria.

Cabido, M., Carranza, M.L., Acosta, A. \& Páez, S. (1991). Contribución al conocimiento fitosociológico del Bosque Chaqueño Serrano en la Provincia de Córdoba, Argentina. Phytocoenologia 19(4): 547-566.

Cabrera, A.L. (1970). La vegetación del Paraguay en el cuadro fitogeográfico de América del sur. Bol. Soc. Argent. Bot. 11: 121-131.

Cabrera, A. L. \& Willink, A. (1973). Biogeografía de América latina. Washington: Organización de los Estados Americanos.

Caetano, S. \& Naciri, Y. (2011). The Biogeography of Seasonally Dry Tropical Forests in South America. In: Dirzo, R., Young, H. \& Mooney, H.A. \& Ceballos, G. (Hrsg.). Seasonally dry tropical forests. Ecology and conservation. Washington: Island Press. S. 23-44. 
Cantero, J.J. (1999). Plant community diversity and habitat relationships in central Argentine grasslands. Diss. Biol. Univ. Tart. 49: 1-165.

Cantero, J.J., León, R., Cisneros, J.M. \& Cantero, A. (1998b). Habitat structure and vegetation relationships in central Argentina salt marsh landscapes. Plant Ecology 137: 79-100.

Carlquist, S. (1983). Intercontinental Dispersal. Sonderbd. naturwiss. Ver. Hamburg (7): 37-47.

Chapman, V.J. (1974). Salt marshes and salt deserts of the world. In Reimold, R.J. \& Queen, W.H. (Hrsg.), Ecology of halophytes. New York: Academic Press. S. 3-19.

CHG (2013). Catalogue des herbiers de Genève (CHG). http://www.ville-ge.ch/musinfo/bd/cjb/chg/.

Contreras, J.R., Contreras, A.O., Ríos, E.E., Rolón, F.J., Delpino, M.A. \& Fornerón, G. (2010). El fenómeno climático de "EL NIÑO". Inundacion y riesgos en el Paraguay. Pilar, Paraguay: Universidad Nacional de Pilar.

Curtis, J.T. \& McIntosh, R.P. (1951). An Upland Forest Continuum in the Prairie-Forest Border Region of Wisconsin. Ecology 32(3): 476-496.

D’oca, M., Morón-Villarreyes, J.A., Lemões, L.S. \& Costa, C. (2012). Fatty acids composition in seeds of the South American glasswort Sarcocornia ambigua. An. Acad. Bras. Cienc. 84(3): 865870.

DGEEC (2013). Anuario Estadístico del Paraguay 2012. Direccion General de Estadística, Encuestas y Censos.

Dinerstein, E., Olson, D.M., Graham, D.I., Webster, A.L., Primm, S.A. \& Bookbinder, M.P. \& Ledec, G. (1995). A Conservation Assessment of the Terrestrial Ecoregions of Latin America and the Caribbean. Washington: World Banik \& WWF.

Ellenberg, H. \& Mueller-Dombois, D. (1967). A key to Raunkiaer plant life forms with revised subdivisions. Ber. Geobot. Inst. ETH, Stiftung Rubel 37: 56-74.

Escurra, C. (1993). Systematics of Ruellia (Acanthaceae) in southern South America. Ann. Miss. Bot. Gard. 80: 787-845.

Eskuche, U. (1992b). Los Espartillares, Un monumento histórico precolombino en el tapiz vegetal del Nordeste Argentino. Corrientes, Argentina: Herbario Humboldtianum, F.A.C.E.N.A. UNNE.

Eskuche, U. (1992a). Sinopsis cenosistemática preliminar de los pajonales mesófilos semi-naturales del nordeste de la Argentina, incluyendo pajonales pampeanos y puntanos. Phytocoenologia 21(3): 237-312.

Esser, G. (1982). Vegetationsgliederung und Kakteenvegetation von Paraguay, Wiesbaden: Steiner [u.a.].

Esser, G. (1984). Natürliche und anthropogen beeinflusste Vegetation in Paraguay. Phytocoenologia 12(2-3): 305-320.

Faggi, A.M. (1985). Las comunidades vegetales de Río Gallegos, Santa Cruz. In: Boelcke, O., Moore, D.M. \& Roig, F.A. (Hrsg.), Transecta botanica de la Patagonia austral. Buenos Aires: Consejo Nacional de Investigaciones Científicas y Técnicas (Argentina), Instituto de la Patagonia (Chile), Royal Society (Gran Bretaña). S. 592-630.

Feldman, S.R., Alzugaray, C. \& Lewis, J.P. (2007). Relación entre la vegetación y el banco de semillas de un espartillar de Spartina argentinensis. Cien. Inv. Agr. 34(1): 41-48.

Feldman, S.R., Bisaro, V. \& Biani, N.B. \& Prado, D.E. (2008). Soil salinity determines the relative abundance of C3/C4 species in Argentinean grasslands. Global Ecol. Biogeogr. 17: 708-714.

Feldman, S.R., Bisaro, V. \& Lewis, J.P. (2009). Análisis discriminante de una comunidad halófila con distintas historias de fuego. Interciencia 34(6): 443-447.

Feldman, S.R. \& Lewis, J.P. (2005). Effects of fire on the structure and diversity of a Spartina argentinensis tall grassland. App. Veg. Sci. 8: 77-84.

Fuentes, A. \& Navarro, G. (2000). Estudio fitosociológico de la vegetacion de una zona de contacto Chaco-Cerrado en Santa Cruz (Bolivia). Lazaroa 21: 73-109. 
Galan de Mera, A. \& Navarro, G. (1992). Comunidades vegetales acuáticas del Paraguay Occidental. Caldasia 17(1): 35-46.

Galán de Mera, A. (2001). Nota sintaxonómica sobre la vegetación del Chaco Boreal. Arnaldoa 8(2): 81-84.

Galán de Mera, A. \& Vicente, J.A. (2006). Aproximación al esquema sintaxonómico de la vegetación de la región del Caribe y América del Sur. Anales de Biología(28): 3-27.

Gehler, E. (1992). Mineralstoffgehalte von Salzböden und Halophyten des bolivianischen Altiplano. Dissertation. Georg-August-Universität, Göttingen.

Giorgis, M.A., Cingolani, A.M., Chiarini, F., Chiapella, J., Barboza, G., Espinar, L.A., Morero, R., Gurvich, D.E., Tecco, P.A., Subils, R. \& Cabido, M. (2011). Composición florística del Bosque Chaqueño Serrano de la Provincia de Córdoba, Argentina. Kurtziana. 36(1): 9-43.

Glatzle, A., Reimer, L., Roth, G. \& Cobo, J. (2006). Dinámica de la napa acuífera salobre a lo largo de gradientes espaciales (campo bajo - monte - pastura). Informe INTTAS.

Glatzle, A., Schultze-Kraft, R. \& Mitlöhner, R. (2000). Potential Role of Native Bush in the Chaco for Mitigation of Dryland Salinity in Grassland. Proc. XVII. Internat. Grassl. Congr. Piracicaba, Brazil.

González Erico, E. (2007). Suelos del Paraguay. Diversidad y origen de los suelos del país. In: SalasDueñas, D.A. \& Facetti, J.F. (Hrsg.). Biodiversidad del Paraguay. Una aproximación a sus realidades. Asunción: Fundación Moisés Bertoni. S. 47-55.

González Gutiérrez, P., Verdecía Góngora, J.L., Leiva, O., Matos, A. \& Peña, R. (2006). Apuntes sobre la flora y la vegetación del sector costero Corinthia-Barrederas, Frank País, Holguín. Rev. Jard. Bot. Nac. 27: 33-45.

Gorham, J.R. (1973). The Paraguayan Chaco and its Rainfall. In: Gorham, J.R. (Hrsg.). Paraguay: ecological essays. Miami: Academy of the Arts and Sciences of the Americas. S. 39-60.

Grassi, E. et al. (2005). Atlas climático del Chaco paraguayo. Loma Plata, Paraguay: Fundación para el Desarrollo Sustentable del Chaco.

Grimm, A.M. \& Tedeschi, R.G. (2009). ENSO and extreme rainfall events in South America. Journal of Climate 22: 1589-1609.

Gutte, P. \& Müller, G.K. (1985). Salzgesellschaften bei Cusco/Perú. Math. Naturwiss. R. 34: 402-409.

Hampel, H. (2000). Untersuchungen zur Dynamik, Struktur und waldbaulichen Behandlung von Wäldern des argentinischen Feuchtchaco. Eschborn: GTZ.

Harder, W., Thiessen H. \& Klassen N. (2004). Libreto de Agua. Colecta, almacenamiento y reciclaje de Agua en el Chaco Central. Loma Plata, Paraguay: Chortizer Komitee LTDA. \& INTTAS.

Hennekens, S.M. \& Schaminée, J. (2001). TURBOVEG, a comprehensive data base management system for vegetation data. J. Veg. Sci. 12: 589-591.

Hilgert, N.I., Pensiero, J.F., Marino, G., Lewis, J.P. \& D'Angelo, C. (2003). Vegetation of the Saladillo Area (Province of Santa Fe) in the South of the Chaco, Argentina. Interciencia 28(9): 512-520.

Hill, M. (1979). TWINSPAN - A FORTRAN program for arranging multivariate data in an ordered two-way-table by classification of the individuals and attributes. Cornell University, Ithaca: Section of Ecology and Systematics.

Hill, M. \& Gauch, H. (1980). Detrended correspondence analysis: An improved ordination technique. Vegetatio 42: 47-58.

Hueck, K. (1966). Die Wälder Südamerikas. Ökologie, Zusammensetzung und wirtschaftliche Bedeutung. Stuttgart: Gustav Fischer Verlag.

Huespe, H.M., Spinzi, L.A., Curiel de Enciso, M.V. \& Henninger, J. (1991). Vegetación y uso de la Tierra de la Region Occidental del Paraguay (Chaco). San Lorenzo, Paraguay: Universidad Nacional de Asunción, Facultad de Ingenieria Agronómica/ Missión Forestal Alemana (GTZ). 
IRIS (2013). Base de datos IRIS. http://www2.darwin.edu.ar/Herbario/Bases/BuscarIris.asp.

Isacch, J.P., Costa, C. S. B., Rodriguez-Gallego, L., Conde, D., Escapa, M., Gagliardini, D.A. \& Iribarne, O.O. (2006). Distribution of saltmarsh plant communities associated with environmental factors along a latitudinal gradient on the south-west Atlantic coast. J. Biogeogr. 33(5): 888-900.

Jacobs, H. (1990). Vegetationsanalytische und strukturelle Untersuchungen einer regengrünen Trockenwaldvegetation im östlichen Bereich des zentralen Chacos unter Berücksichtigung des Einflusses der Viehweide. Diplomarbeit an der Forstlichen Fakultät. Georg-August-Universität. Johnston, I.M. (1940). The floristic significance of shrubs common to North and South American deserts. J. Arnold Arboretum 21: 356-363.

Junker, M. \& Hoyer, M. von (1998). Verbesserung der Trinkwasserversorgun für die armen Bevölkerungsgruppen im Chaco von Paraguay. Z. angew. Geol. 44(2): 100-107.

Karlin, M.S., Bachmeier, O.A., Dalmasso, A., Sayago, J.M. \& Sereno, R. (2011). Environmental Dynamics in Salinas Grandes, Catamarca, Argentina. Arid Land Research and Management 25(4): 328-350.

Kataria, T.N. (2008). Physiology of high and saline plants. New Delhi: Pearl Books.

Kaur, B., Gupta, S.R. \& Singh, G. (2002). Bioamelioration of a sodic soil by silvopastoral systems in northwestern India. Agroforestry Systems 54: 13-20.

KEW (2013). Kew Herbarium Catagolue. http://apps.kew.org/herbcat/navigator.do.

Kruck, W., Barboza, F., Carlini, A., Hoffmann, R., Medina Netto, A. \& Mereles, F. (1998). Chaco Schutz und nachhaltige Nutzung. Proyecto Sistema Ambiental Chaco. Erfassung, Bewertung und Empfehlungen zum Schutz des Naturraumpotentials der Westregions Paraguays, San Lorenzo, Paraguay: Ministerio de Agricultura y Ganaderia (MAG), Dirección de Ordenamiento Ambiental (DOA) \& Bundesanstalt für Geowissenschaften und Rohstoffe (BGR).

La Peyre, M. K. \& Rowe, S. (2003). Effects of salinity changes on growth of Ruppia maritima L. Aquatic Botany 77(3): 235-241.

Lamprecht, H. (1986). Waldbau in den Tropen. Die tropischen Waldökosysteme und ihre Baumarten Möglichkeiten und Methoden zu ihrer nachhaltigen Nutzung. Hamburg: Parey.

Le Houérou, H. (1993). Salt-tolerant plants for the arid regions of the Mediterranean isoclimatic zone. In: Lieth, H. \& Al Masoom, A. A. (Hrsg.), Towards the rational use of high salinity tolerant plants. Proceedings of the First ASWAS Conference, December 8-15, 1990 at the United Arab Emirates University, Al Ain, United Arab Emirates. Dordrecht: Springer. S. 403-422.

Lewis, J.E., Pire E.F., Prado, D., Stofella, S.L., Franceschi, E.A. \& Carnevale, N.J. (1990). Plant communities and phytogeographical position of a large depression in the Great Chaco, Argentina. Vegetatio 86: 25-38.

Leyer, I. \& Wesche, K. (2007). Multivariate Statistik in der Ökologie - Eine Einführung. Berlin/Heidelberg/New York: Springer.

López, J.A., Little, E.L., Ritz, G.F., Rombold, J.S. \& Hahn, W.J. (2002). Arboles comunes del Paraguay. Ñande yvyra mata kuera. San Lorenzo, Paraguay: Facultad de Ciencias Agrarias, U.N.A. \& Cuerpo de Paz.

López, R., Alcázar, D.L. \& Marcía M.J (2006). The arid and dry plant formations of South America and their floristic connections: New data, new interpretation? Darwiniana 44(1): 18-31.

López, R.P. \& Beck S. (2002). Phytogeographical affinities and life form composition of the Bolivian Prepuna. Candollea 57(1): 77-96.

Lüders, R. (1962). Böden des Chaco Boreal von Paraguay. Geol. Jb. 81: 82-134.

Medina, E., Francisco, A.M., Wingfield, R. \& Casañas, O.L. (2008). Halofitismo en plantas de la Costa Caribe de Venezuela: Halófitas y Halotolerantes. Acta Bot. Venez. 31(1): 49-80. 
Meloni, D.A., Gulotta, M.R. \& Martínez, C.A. (2008). Prosopis ruscifolia Griseb. (vinal) tolera concentraciones salinas equivalentas al agua de mar y excluye iones tóxicos de la parte aérea. Quebracho 16: 32-40.

Menghi, M. \& Del Sueldo, R. \& Carelli, H. (2001). Relación entre la diversidad y biomasa en comunidades herbáceas del valle de inundación del Río Dulce (Argentina Central). Importancia para su manejo. Pastos 31(2): 217-232.

Mereles, F. (1998). Etude de la flore et de la végétatión de la mosaïque foret-savane palmeraie dans le Chaco Boreal, Paraguay. Thèse de doctorat. Faculté des Sciences, Université de Genéve.

Mereles, F. (2004). Los humedales del Paraguay: principales tipos de vegetación. In: Salas, D. \& Mereles F. \& Yanosky A. (Hrsg.). Humedales de Paraguay. Asunción, Paraguay: Comité Nacional de Humedales. S. 67-88.

Mereles, F. (2005). Una aproximación al conocimiento de las formaciones vegetales del Chaco Boreal, Paraguay. Rojasiana 6(2): 5-48.

Mereles, F. (2007). La diversidad vegetal en el Paraguay. In: Salas-Dueñas, D.A. \& Facetti, J.F. (Hrsg.). Biodiversidad del Paraguay. Una aproximación a sus realidades. Asunción: Fundación Moisés Bertoni. S. 89-105.

Mereles, F. \& Degen, R. (1997). Leñosas colonizadoras e indicadoras de sitios modificados en el Chaco Boreal, Paraguay. Rojasiana 4(1): 25-83.

Mereles, F. \& Degen, R. (1998). Formaciones vegetales del Chaco Boreal Paraguayo. In: Kruck, W. (Hrsg.). Proyecto Sistema Ambiental del Chaco. Inventario, evaluación y recomendaciones para la proteccion de los espacios naturales en la Región Occidental del Paraguay (II). San Lorenzo, Paraguay: Ministerio de Agricultura y Ganaderia (MAG), Dirección de Ordenamiento Ambiental (DOA) \& Bundesanstalt für Geowissenschaften und Rohstoffe (BGR). S. 75-87.

Mereles, F. \& Rodas, O. (2009). El proceso de fragmentación y reducción de hábitat en el Chaco paraguayo y sus efectos sobre la biodiversidad. In: Morello, J. \& Rodríguez, A.F. (Hrsg.), El Chaco sin bosques. La Pampa o el desierto del futuro. Buenos Aires: Orientación Gráfica Editora. S. 273-292.

Mitloehner, R. \& Koepp, R. (2007). Bioindicator capacity of trees towards dryland salinity. Trees 21(4): 411-419.

Mitlöhner, R. (1990). Die Konkurrenz der Holzgewächse im regengrünen Trockenwald des Chaco Boreal, Paraguay. Göttingen: E. Goltze.

Mitlöhner, R. (1998). Pflanzeninterne Potentiale als Indikatoren für den tropischen Standort. Aachen: Shaker.

Montesinos-Tubée, D.B. (2012). Vegetacion halófila de tres localidades andinas en la vertiente pacífica del sur de Perú. Chloris chilensis 15(2).

Moore, M.J., Tye, A. \& Jansen, R.K. (2006). Patterns of long-distance dispersal in Tiquilia subg. Tiquilia (Boraginaceae): implications for the origins of amphitropical disjuncts and Galapagos Islands endemics. Amer. J. Bot. 93: 1163-1177.

Moreira-Muñoz, A. (2007). Geography of Chile. An Essay on Postmodern Biogeography. Dissertation. Friedrich-Alexander-Universität.

Morello, J. \& Saravia, C. (1959). El bosque chaqueño I. Paisaje primitivo, paisaje natural y paisaje cultural en el oriente de Salta. Rev. Agr. Noroeste Arg. 3(1-2): 5-81.

Navarro, G. (1997). Contribución a la clasificación ecológica y florística de los bosques de Bolivia. Rev. Bol. Ecol. 2: 3-37.

Navarro, G. (2005). Unidades de vegetación de la Reserva de Biósfera del Chaco Paraguayo. In: Rumiz, D I. \& L. Villalba (Hrsg.). Unidades ambientales de la reserva de la biósfera del Chaco Paragayo. Santa Cruz de la Sierra, Bolivia: Wildlife Conservation Society \& Fundación para el Desarrollo Sustentable del Chaco. S. 25-50. 
Navarro, G. \& Maldonado, M. (2002). Geografía ecológica de Bolivia. Vegetación y ambientes acuáticos. Cochabamba, Bolivia: Centro de Ecología Simón I. Patiño, Departamento de Difusión.

Navarro, G., Molina, J.A. \& Pérez de Molas, L. (2006). Classification of the forests of the northern Paraguayan Chaco. Phytocoenologia 36(4): 473-508.

Navarro, G., Molina, J.A. \& Vega, S. (2011). Soil factors determining the change in forests between dry and wet Chacos. Flora 206(2): 136-143.

Navarro, G. \& Fuentes, A. (1999). Geobotánica y sistemas ecológicos de paisaje en el Gran Chaco de Bolivia. Rev. Bol. Ecol. 5: 25-50.

Nebbia, A.J., Zalba \& S.M. (2007). Comunidades halófilas de la costa de la Bahía Blanca (Argentina). Caracterización, mapeo y cambios durante los últimos cincuenta años. Bol. Soc. Argent. Bot. 42(3-4): 261-271.

NHM (2013). Botany collection database. http://www.nhm.ac.uk/research-curation/scientificresources/collections/botanical-collections/botany-specimen-database/.

NYBG (2013). Virtual Herbarium. http://sciweb.nybg.org/science2/vii2.asp.

Oakley, L... \& Prado, D.E. (2011). El Dominio de los Bosques Secos Estacionales Neotropicales y la presencia del Arco Pleistocénico en la República del Paraguay. Rojasiana 10(1): 55-75.

Pérez de Molas, L. (1994). Estructura, composición florística y dinámica de la sabana arbolada en paleocauces colmatados del Chaco paraguayo. Magisterarbeit in Forstwissenschaften der Tropen. Georg-August-Universität.

Pott, A., Oliveira, A., Damasceno-Junior, G.A. \& Silva, J. (2011). Plant diversity of the Pantanal wetland. Braz. J. Biol. 71(1): 265-273.

Prado, D. (1993a). What is the Gran Chaco vegetation in South America? I. A review. Contribuion to te study of flora and vegetation of the Chaco. V. Candollea 48(1): 145-172.

Prado, D. (1993b). What is the Gran Chaco vegetation in South America? II. A redefinition. Contribution to the study of the flora and vegetation of the Chaco. VII. Candollea 48(2): 615-629.

Prado, D. (2000). Seasonally dry forests of tropical South America: From forgotten ecosystems to a new phytogeographic unit. Edinb. J. Bot. 57(3): 437-461.

Prado, D. \& Gibbs, P. (1993). Patterns of species distributions in the dry seasonal forests of South America. Ann. Miss. Bot. Gard. 80(4): 902-927.

Putzer, H. (1962). Geologie von Paraguay. Berlin: Gebrüder Borntraeger.

Ragonese, A.E. (1951). La vegetación de la República Argentina II. Estudio fitosociológico de las Salinas Grandes. Rev. Inv. Agric. 5(1-2): 1-233.

Ragonese, A.E \& Covas, G. (1947). La flora halófila del Sur de la Provincia de Santa Fe (República Argentina). Darwiniana 7(3): 401-498.

Ragonese, A.E. \& Piccinini, B. (1977). Consideraciones sobre la vegetación de las Salinas de Mascasín (La Rioja-San Juan, República Argentina). Darwiniana 21(1): 49-60.

Ramella, L. (2008). Catalogus Hasslerianus I. Fl. Paraguay(Serie Especial 4), 11-365.

Ramella, L. (2009). Catalogus Hasslerianus II. Fl. Paraguay(Serie Especial 5), 13-255.

Ramella, L. (2010). Catalogus Hasslerianus III. Fl. Paraguay(Serie Especial 6), 13-234.

Ramella, L. (2011). Catalogus Hasslerianus IV. Fl. Paraguay(Serie Especial 7), 13-280.

Ramella, L. \& Spichiger, R. (1989). Interpretación preliminar del medio físico y de la vegetación del Chaco Boreal. Contribución al estudio de la flora y la vegetación del Chaco I. Candollea 44(2): 639-680.

Raven, P.H. (1963). Amphitropical relationships in the floras of North and South America. Q. Rev. Biol. 38: 151-177.

Rempel, E. (2007). Análisis estructural en Parcela Permanente de Medición de un Bosque semicaducifolio "Palosantal-Labonal", Reserva Natural Privada de la Cooperativa Fernheim 
"Laguna Porá", Dpto. Presidente Hayes, Paraguay. Tesis en Ingeniería Forestal. Facultad de Ciencias Agrarias, Universidad Nacional de Asunción.

Rieger, W. (1976). Vegetationskundliche Untersuchungen auf der Guajira-Halbinsel (NordostKolumbien). Giessen.

Ríos, N., Cejas, M. \& Maldonado, M. (2008). El Vinal (Prosopis ruscifolia Griseb.) una especie importante en el Gran Chaco Americano, Argentina. Foresta Veracruzana 10(2): 17-26.

Roig, F.A., Anchorema, J., Dollenz, O. \& Faggi, A.M. \& Méndez, E. (1985). Las comunidades vegetales de la transecta botánica de la Patagonia Austral. Primera parte: La Vegetación del área continental. In: Boelcke, O., Moore, D.M. \& Roig, F.A. (Hrsg.), Transecta botanica de la Patagonia austral. Buenos Aires: Consejo Nacional de Investigaciones Científicas y Técnicas (Argentina), Instituto de la Patagonia (Chile), Royal Society (Gran Bretaña). S. 350-456.

Roleček, J., Tichý, L., Zelený, D. \& Chytrý, M. (2009). Modified TWINSPAN classification in which the hierarchy respects cluster heterogeneity. J. Veg. Sci. 20: 596-602.

Roth, I. \& Giménez, A.M. (1997). Argentine Chaco forests. Dendrology, tree structure, and economic use. 1. The semiarid Chaco. Berlin/Stuttgart: G. Borntraeger.

Roth, I. \& Giménez, A.M. (2006). Argentine Chaco forests. Dendrology, tree structure, and economic use. 2. The humid Chaco. Berlin/Stuttgart: G. Borntraeger.

Ruiz Posse, E., Karlin, U.O., Buffa, E., Karlin, M., Gial Levra, C. \& Castro, G. (2007). Ambientes de las Salinas Grandes de Catamarca, Argentina. Multequina 16: 123-137.

Sarmiento, G. (1975). The dry plant formations of South America and their floristic connections. J. Biogeogr. 2: 233-251.

Schinini, A. (2008). Nueva cita para Paraguay - Tillandsia mereliana Schinini, Nov. Spec. Rojasiana 8(1): 73-76.

Soil Survey Staff (1996). Soil Survey Laboratory Methods Manual. Washington, DC.: U.S. Department of Agriculture, Natural Resources Conservation Service.

Solbrig, O.T. (1972). The floristic disjunctions between the Monte in Argentina and the Sonoran Desert in Mexico and the United States. Ann. Miss. Bot. Gard. 59: 605-614.

Solomon, S., Qin, D., Manning, M., Chen, Z., Marquis, M., Averyt, K.B., Tignor, M. \& Miller, H.L. (Hrsg.) (2007). Climate change 2007. The physical science basis : contribution of Working Group I to the Fourth Assessment Report of the Intergovernmental Panel on Climate Change. Cambridge, New York: Cambridge University Press.

Spichiger, R., Bise, B., Calenge, C. \& Chatelain C. (2006). Biogeography of the Forests of the Paraguay-Paraná Basin. In Pennington, T., Lewis, G.P. \& Ratter, J.A. (Hrsg.), Neotropical savannas and dry forests. Diversity, biogeography, and conservation. Boca Raton, FL: CRC Press. S. 193-211.

Spichiger, R., Calenge, C. \& Bise B. (2004). Geographical zonation in the Neotropics of tree species characteristic of the Paraguay-Paraná Basin. J. Biogeogr. 31: 1489-1501.

Spichiger, R., Calenge, C. \& Bise B. (2005). Discriminant analysis of the spatial distribution of plant species occurrences : II. Distribution of major tree communities in Paraguay. Candollea 60(2): 577-593.

Spichiger, R., Palese R., Chautems A. \& Ramella L. (1995). Origen, affinities and diversity hot spots of the Paraguayan dendrofloras. Candollea 50(2): 515-537.

Spichiger, R., Ramella, L., Palese, R. \& Mereles F. (1991). Proposición de leyenda para la Proposición de leyenda para la cartografía de las formaciones vegetales del Chaco paraguayo. Contribución al estudio de la flora y la vegetación del Chaco III. Candollea 46(2): 541-564.

Stevens, P.F. (2001 [continuously updated]). Angiosperm Phylogeny Website. http://www.mobot.org/MOBOT/research/APweb/.

Takhtajan, A. (1986). Floristic Regions of the World. University of California Press. 
Taleisnik, E. \& López, D. (2011). Leñosas perennes para ambientes afectados por salinidad. Una sinopsis de la contribución argentina a este tema. Ecología Austral 21: 3-14.

Teillier, S. \& Becerra, P. (2003). Flora y vegetación del salar de Ascotán, Andes del Norte de Chile. Gayana Bot. 60(2): 114-122.

ter Braak, C. \& Šmilauer, P. (2002). CANOCO Reference Manual and CanoDraw for Windows User's Guide. Wageningen/České Budějovice: Biometrics.

ter Braak,, C.J.F. (1987). The analysis of vegetation-environment relationships by Canonical Correspondence Analysis. Vegetatio 69: 69-77.

The Nature Conservancy (TNC), Fundación Vida Silvestre Argentina (FVSA), Fundación para el Desarrollo Sustentable del Chaco (DeSdelChaco) \& Wildlife Conservation Society Bolivia (WCS) (2005). Evaluación Ecorregional del Gran Chaco Americano / Gran Chaco Americano Ecorregional Assessment. Buenos Aires: Fundación Vida Silvestre Argentina.

Therburg, A. (1997). Ökologie der Halophytenvegetation in der Provinz Mendoza, Argentinien (Monte-Formation). Berlin, Stuttgart: Cramer in der Gebr.-Borntraeger-Verl.-Buchh.

Tichý, L. (2002). JUICE, software for vegetation classification. J. Veg. Sci. 13: 451-453.

Tortorelli, L.A. (1966). Formaciones forestales y maderas del Paraguay. Asunción: Facultad de Agronomia y Veterinaria, Universidad de Asunción.

Tropicos (2013). Tropicos.org. http://www.tropicos.org/.

Udvardy, M. (1975). A classification of the biogeographical provinces of the world. Morges, Switzerland.

Ungar, I.A. (1972). The vegetation of inland saline marshes of North America, North of Mexico. In Maarel, E. van der \& Tüxen, R. (Hrsg.), Grundlagen und Methoden in der Pflanzensoziologie. Den Haag: Verlag Dr. Junk N.V. S. 397-411.

Vareschi, V. (1980). Vegetationsökologie der Tropen. Stuttgart: Ulmer.

Vervoorst, F.B. (1967). La vegetación de la República Argentina VII. Las comunidades vegetales de la Depresión del Salado (Provincia de Buenas Aires). Buenos Aires: INTA.

Vogt, C. (2011). Composición de la Flora Vascular del Chaco Boreal, Paraguay I. Pteridophyta y Monocotiledoneae. Steviana 3: 13-47.

Vogt, C. (2012). Composición de la Flora Vascular del Chaco Boreal, Paraguay II. Dicotyledoneae: Acanthaceae-Fabaceae. Steviana 4: 65-116.

Vogt, C. (2013). Composición de la Flora Vascular del Chaco Boreal, Paraguay III. Dicotyledoneae: Gesneriaceae - Zygophyllaceae. Steviana 5: 5-40.

Walter, H. \& Breckle, S.W. (1984). Ökologie der Erde. Band 2: Spezielle Ökologie der Tropischen und Subtropischen Zonen. Stuttgart: Fischer.

Werger, M. (1973). Las disyunciones anfitrópicas en las floras xerofíticas norte y sudamericanas. Darwiniana 18(1-2): 9-18.

Wiebe, S. (2003). Abhängigkeiten der Bodenversalzung von pedologischen, klimatischen, hydrologischen und anthropogenen Faktoren im semiariden Klima des Chaco Boreal, Paraguay. Osnabrück: Der Andere Verlag.

Wiens, F. (1998). Evaluación económica del uso de la tierra al este de Loma Plata, Chaco Central Oriental, Problemas de salinización en suelos de monte en una zona de transición. In: Kruck, W. (Hrsg.), Proyecto Sistema Ambiental del Chaco. Inventario, evaluación y recomendaciones para la proteccion de los espacios naturales en la Región Occidental del Paraguay (IV). San Lorenzo, Paraguay: Ministerio de Agricultura y Ganaderia (MAG), Dirección de Ordenamiento Ambiental (DOA) \& Bundesanstalt für Geowissenschaften und Rohstoffe (BGR). S. 103-137.

Wilmanns, O. (1998). Ökologische Pflanzensoziologie. Eine Einführung in die Vegetation Mitteleuropas. Heidelberg, Wiesbaden: Quelle und Meyer. 
Yensen, N.P. (2008). Halophyte uses for the twenty-first century. In: Khan, M.A. \& Weber, D.J.

(Hrsg.). Ecophysiology of high salinity tolerant plants. Dordrecht, The Netherland: Springer. S. 367-396.

Zak, M.R. \& Cabido, M. (2002). Spatial patterns of the Chaco vegetation of central Argentina: Integration of remote sensing and phytosociology. App. Veg. Sci. 5: 213-226.

Zuloaga, F.O., Morrone, O., Belgrano M.J., Marticorena, C. \& Marchesi, E. (Hrsg.) (2008). Catálogo de las plantas vasculares del Cono Sur. (Argentina, Sur de Brasil, Chile, Paraguay y Uruguay). St. Louis: Missouri Botanical Garden Press.

Zuloaga, F.O., Morrone, O. \& Rodríguez, D. (1999). Análisis de la biodiversidad en plantas vasculares de la Argentina. Kurtziana 27(1): 17-167. 


\section{Anhang}

\section{Anhang zum 3. Kapitel}

Anhang 3-1.: Liste der Pflanzenfamilien im Untersuchungsgebiet.......................105

Anhang 3-2.: Artenliste des Untersuchungsgebietes.................................107

\section{Anhang zum 4. Kapitel}

Anhang 4-1: Liste der Probeflächen in den Salzmarschen.............................113

Anhang 4-2: Artenliste der Salzmarschen.........................................116

Anhang 4-3: Bodenparameter der Salzmarschen...................................117

Anhang 4-4: Log-file der Korrespondenzanalyse (DCA) zur Ermittlung der

Gradientenlänge.............................................................19

Anhang 4-5: Log-file der Hauptgradientenanalyse (PCA) des Leptochloa-Sesuvium-

Sarcocornia Komplexes.......................................................119

Anhang 4-6: Log-file der indirekten Gradientenanalyse (DCA) der Bodenparameter.........119

Anhang 4-7: Log-file der direkten Gradientenanalyse (CCA) der Bodenparameter...........119

\section{Anhang zum 5. Kapitel}

Anhang 5-1: Liste der Probeflächen in der Gehölzvegetation...........................121

Anhang 5-2: Synoptische Tabelle aller vorkommenden Arten in den Gehölzgesellschaften.126

Anhang 5-3: Bodenparameter der Gehölzvegetation..................................134

Anhang 5-4: Log-file der Korrespondenzanalyse (DCA) der Gesellschaften 10 bis $16 . . . . . .136$

Anhang 5-5: Log-file der Hauptgradientenanalyse (PCA) der Waldgesellschaften...........136

Anhang 5-6: Log-file der indirekten Gradientenanalyse (DCA) der Bodenparameter.........136

Anhang 5-7: Log-file der direkten Gradientenanalyse (CCA) der Bodenparameter...........137 
Anhang 3-1.: Liste der Pflanzenfamilien im Untersuchungsgebiet. Angaben zur Anzahl der Gattungen, Arten und dem jeweiligen floristischen Status der Arten: Nat (heimisch), EndCS (endemisch vom Conosur), EndGC (endemisch vom Gran Chaco), End (endemisch vom paraguayischen Chaco), Natur (eingebürgerte Arten).

\begin{tabular}{|c|c|c|c|c|c|c|c|}
\hline Familien & Gattungen & Arten & Nat & EndCS & EndGC & End & Natur \\
\hline Acanthaceae & 5 & 10 & 9 & 1 & & & \\
\hline Achatocarpaceae & 1 & 1 & 1 & & & & \\
\hline Aizoaceae & 2 & 2 & 2 & & & & \\
\hline Amaranthaceae & 8 & 12 & 11 & & & & 1 \\
\hline Anacardiaceae & 2 & 3 & 3 & & & & \\
\hline Anemiaceae & 1 & 1 & 1 & & & & \\
\hline Apocynaceae & 7 & 10 & 10 & & & & \\
\hline Araceae & 1 & 1 & 1 & & & & \\
\hline Arecaceae & 2 & 2 & 2 & & & & \\
\hline Asparagaceae & 1 & 1 & 1 & & & & \\
\hline Asteraceae & 17 & 21 & 14 & 6 & & 1 & \\
\hline Bignoniaceae & 4 & 7 & 7 & & & & \\
\hline Boraginaceae & 3 & 7 & 5 & 1 & & 1 & \\
\hline Bromeliaceae & 6 & 16 & 13 & & 2 & 1 & \\
\hline Cactaceae & 11 & 16 & 13 & 1 & 1 & 1 & \\
\hline Cannabaceae & 1 & 1 & 1 & & & & \\
\hline Capparaceae & 4 & 4 & 4 & & & & \\
\hline Caricaceae & 1 & 1 & & & 1 & & \\
\hline Caryophyllaceae & 2 & 2 & 2 & & & & \\
\hline Celastraceae & 2 & 2 & 1 & 1 & & & \\
\hline Cleomaceae & 1 & 1 & 1 & & & & \\
\hline Commelinaceae & 2 & 2 & 2 & & & & \\
\hline Convolvulaceae & 5 & 8 & 8 & & & & \\
\hline Cucurbitaceae & 1 & 1 & 1 & & & & \\
\hline Сyperaceae & 4 & 6 & 6 & & & & \\
\hline Erythroxylaceae & 1 & 1 & 1 & & & & \\
\hline Euphorbiaceae & 9 & 16 & 15 & 1 & & & \\
\hline Fabaceae & 27 & 43 & 36 & & 6 & 1 & \\
\hline Hydnoraceae & 1 & 1 & 1 & & & & \\
\hline Lamiaceae & 1 & 1 & 1 & & & & \\
\hline Loranthaceae & 1 & 1 & 1 & & & & \\
\hline Malpighiaceae & 2 & 2 & 2 & & & & \\
\hline Malvaceae & 12 & 21 & 20 & & 1 & & \\
\hline Molluginaceae & 1 & 1 & 1 & & & & \\
\hline Moraceae & 1 & 1 & 1 & & & & \\
\hline Nyctaginaceae & 3 & 5 & 3 & & 1 & & 1 \\
\hline Orchidaceae & 1 & 1 & 1 & & & & \\
\hline Oxalidaceae & 1 & 1 & 1 & & & & \\
\hline Passifloraceae & 2 & 5 & 5 & & & & \\
\hline Phytolaccaceae & 1 & 1 & 1 & & & & \\
\hline Plantaginaceae & 3 & 3 & 3 & & & & \\
\hline Poaceae & 19 & 25 & 22 & & & & 3 \\
\hline Polygonaceae & 2 & 3 & 3 & & & & \\
\hline Polypodiaceae & 1 & 1 & 1 & & & & \\
\hline Pontederiaceae & 1 & 1 & 1 & & & & \\
\hline Portulaccaceae & 1 & 4 & 2 & 1 & & & 1 \\
\hline Pteridaceae & 1 & 1 & 1 & & & & \\
\hline Rhamnaceae & 1 & 1 & 1 & & & & \\
\hline Rubiaceae & 3 & 3 & 3 & & & & \\
\hline Ruppiaceae & 1 & 1 & 1 & & & & \\
\hline Rutaceae & 1 & 1 & 1 & & & & \\
\hline Santalaceae & 2 & 2 & 2 & & & & \\
\hline Sapindaceae & 3 & 4 & 4 & & & & \\
\hline Sapotaceae & 1 & 1 & 1 & & & & \\
\hline Selaginellaceae & 1 & 1 & 1 & & & & \\
\hline
\end{tabular}




\begin{tabular}{lrrrrrrr} 
Familien & Gattungen & Arten & Nat & EndCS & EndGC & End & Natur \\
\hline Simaroubaceae & 1 & 1 & 1 & & & & \\
Solanaceae & 5 & 13 & 11 & 2 & & \\
Talinaceae & 1 & 3 & 1 & & & \\
Ulmaceae & 1 & 1 & 1 & & & \\
Verbenaceae & 4 & 5 & 5 & & & \\
Vitaceae & 1 & 1 & 1 & & & \\
Ximeniaceae & 1 & 1 & 1 & & & \\
Zygophyllaceae & 1 & 2 & & & 2 & & \\
\hline Total: 63 & $\mathbf{2 1 4}$ & $\mathbf{3 1 8}$ & $\mathbf{2 7 7}$ & $\mathbf{1 4}$ & $\mathbf{1 4}$ & $\mathbf{5}$ & $\mathbf{8}$
\end{tabular}


Anhang 3-2.: Artenliste des Untersuchungsgebietes. Daten zur Lebensform (LF), floristischen Satus (STAT), phytogeographischer Einordnung und Verbreitung der Arten (DISSPEC) und der Gattungen (DISGEN) so wie zur Einordnung nach dem Niederschlagsgradient im paraguayischen Chaco (NG). Abkürzungsverzeichnis der Lebensformen, des floristischen Status und der phytogeographischen Elemente in Kapitel 8.1. Abkürzungen des Niederschlagsgradienten in Tab. 3-1.

\begin{tabular}{|c|c|c|c|c|c|c|}
\hline Familie & Art & LF & STAT & DISSPEC & DISGEN & NG \\
\hline Acanthaceae & Carlowrightia sulcata & H scap & ENDCS & SURAMA & AMER & $\mathrm{H}, \mathrm{S}$ \\
\hline Acanthaceae & Dicliptera squarrosa & Ch suff/herb & NAT & SURAMA & KOS & $\mathrm{H}$ \\
\hline Acanthaceae & Justicia corumbensis & Ch suff & NAT & CHA & KOS & $\mathrm{H}$ \\
\hline Acanthaceae & Justicia dumetorum & $\mathrm{P}$ herb & NAT & CHA & KOS & $\mathrm{H}, \mathrm{S}$ \\
\hline Acanthaceae & Justicia squarrosa & $\mathrm{CH}$ suff & NAT & CHA & KOS & A, S \\
\hline Acanthaceae & Justicia xylosteoides & P caesp & NAT & CHA & KOS & A, S \\
\hline Acanthaceae & Dyschoriste venturii & Ch suff & NAT & CHA & PAN & $\mathrm{S}$ \\
\hline Acanthaceae & Ruellia erythropus & Ch suff & NAT & AMPHI & PAN & $\mathrm{H}, \mathrm{S}$ \\
\hline Acanthaceae & Ruellia hygrophila & $\mathrm{H}$ ros & NAT & CHA & PAN & $\mathrm{H}, \mathrm{S}$ \\
\hline Acanthaceae & Ruellia simplex & Ch herb & NAT & AMPHI & PAN & $\mathrm{A}, \mathrm{H}, \mathrm{S}$ \\
\hline Achatocarpaceae & Achatocarpus praecox & P scap & NAT & DOMNSTW & NEO & $\mathrm{A}, \mathrm{H}, \mathrm{S}$ \\
\hline Aizoaceae & Sesuvium portulacastrum & H scap & NAT & PAN & PAN & $\mathrm{H}, \mathrm{S}$ \\
\hline Aizoaceae & Trianthema portulacastrum & $\mathrm{T}$ succ & NAT & PAN & PAN & $\mathrm{H}, \mathrm{S}$ \\
\hline Amaranthaceae & Alternanthera kurtzii & Ch herb/H rept & NAT & DOMCHA & KOS & A, H, S \\
\hline Amaranthaceae & Alternanthera nodifera & Ch suff & NAT & CHA & KOS & $\mathrm{S}$ \\
\hline Amaranthaceae & $\begin{array}{l}\text { Alternanthera paronychioides ssp. } \\
\text { chacoënsis }\end{array}$ & H rept & NAT & DOMCHA & KOS & $\mathrm{H}, \mathrm{S}$ \\
\hline Amaranthaceae & Amaranthus hybridus & T scap & NATUR & AMER & KOS & A, S \\
\hline Amaranthaceae & Amaranthus standleyanus & T scap & NAT & DOMCHA & KOS & A, S \\
\hline Amaranthaceae & Amaranthus viridis & T scap & NAT & KOS & KOS & $\mathrm{H}, \mathrm{S}$ \\
\hline Amaranthaceae & Atriplex eximia & Ch suff & NAT & CHA & KOS & $\mathrm{S}$ \\
\hline Amaranthaceae & Chenopodium pilcomayense & T scap & NAT & CHA & KOS & A, S \\
\hline Amaranthaceae & Sarcocornia ambigua & Ch succ & NAT & DISNEO & KOS & $S$ \\
\hline Amaranthaceae & Heterostachys ritteriana & Ch frut & NAT & DISNEO & DISNEO & $\mathrm{S}$ \\
\hline Amaranthaceae & Holmbergia tweedii & $\mathrm{P}$ herb & NAT & DOMCHA & DOMCHA & $\mathrm{S}$ \\
\hline Amaranthaceae & Gomphrena perennis & H caesp & NAT & DOMCHA & NEO & $\mathrm{H}, \mathrm{S}$ \\
\hline Anacardiaceae & Astronium fraxinifolium & P scap & NAT & NEO & NEO & $\mathrm{S}$ \\
\hline Anacardiaceae & Schinopsis cornuta & P scap & NAT & CHA & SURAMA & A, S \\
\hline Anacardiaceae & Schinopsis lorentzii & P scap & NAT & CHA & SURAMA & A, S \\
\hline Anemiaceae & Anemia tomentosa var. anthriscifolia & H caesp & NAT & SURAMA & PAN & A, S \\
\hline Apocynaceae & Aspidosperma quebracho-blanco & P scap & NAT & DOMCHA & NEO & A, H, S \\
\hline Apocynaceae & Aspidosperma triternatum & P scap & NAT & CHA & NEO & $\mathrm{H}, \mathrm{S}$ \\
\hline Apocynaceae & Funastrum clausum & L suff & NAT & NEO & NEO & A, H, S \\
\hline Apocynaceae & Mandevilla angustifolia & L herb & NAT & CHA & NEO & $\mathrm{S}$ \\
\hline Apocynaceae & Oxypetalum arnottianum & L suff/herb & NAT & DOMNSTW & NEO & $\mathrm{S}$ \\
\hline Apocynaceae & Marsdenia castillonii & L suff & NAT & VORAND & PAN & A, S \\
\hline Apocynaceae & Araujia herzogii & L herb & NAT & VORAND & SURAMA & A, S \\
\hline Apocynaceae & Araujia odorata & L herb & NAT & SURAMA & SURAMA & A, H, S \\
\hline Apocynaceae & Araujia variegata & L herb & NAT & VORAND & SURAMA & A, H, S \\
\hline Apocynaceae & Schubertia grandiflora & L herb & NAT & SURAMA & SURAMA & $\mathrm{H}, \mathrm{S}$ \\
\hline Araceae & Spathicarpa hastifolia & G rhiz & NAT & DOMNSTW & DOMNSTW & $\mathrm{S}$ \\
\hline Arecaceae & Copernicia alba & P ros & NAT & CHA & DISNEO & $\mathrm{H}, \mathrm{S}$ \\
\hline Arecaceae & Trithrinax schyzophylla & P ros & NAT & CHA & SURAMA & $\mathrm{H}, \mathrm{S}$ \\
\hline Asparagaceae & Herreria montevidensis & L suff & NAT & SURAMA & SURAMA & $\mathrm{H}, \mathrm{S}$ \\
\hline Asteraceae & Baccharis salicifolia & P caesp & NAT & AMER & AMER & A, S \\
\hline Asteraceae & Baccharis trinervis & $P$ ceasp & NAT & NEO & AMER & $\mathrm{H}, \mathrm{S}$ \\
\hline Asteraceae & Grindelia aff. puberula & P caesp & ENDCS & SURAMA & AMER & $\mathrm{H}$ \\
\hline Asteraceae & Pectis odorata & T scap & NAT & CHA & AMER & $\mathrm{S}$ \\
\hline Asteraceae & Trixis divaricata & Ch suff & NAT & NEO & AMER & A, H, S \\
\hline Asteraceae & Eupatorium catarium & Ch herb & NAT & SURAMA & KOS & $\mathrm{A}, \mathrm{H}, \mathrm{S}$ \\
\hline Asteraceae & Eupatorium christieanum & P herb & ENDCS & DOMNSTW & KOS & $\mathrm{H}, \mathrm{S}$ \\
\hline Asteraceae & Eupatorium patens & P caesp & NAT & DOMCHA & KOS & $\mathrm{H}, \mathrm{S}$ \\
\hline Asteraceae & Vernonia niederleinii & $\mathrm{P}$ herb & ENDCS & SURAMA & KOS & $\mathrm{S}$ \\
\hline Asteraceae & Cyclolepis genistoides & P caesp & NAT & DOMCHA & DOMCHA & $\mathrm{H}, \mathrm{S}$ \\
\hline
\end{tabular}




\begin{tabular}{|c|c|c|c|c|c|c|}
\hline Familie & Art & LF & STAT & DISSPEC & DISGEN & NG \\
\hline Asteraceae & Noticastrum acuminatum & Ch herb & ENDCS & DOMCHA & NEO & S \\
\hline Asteraceae & Porophyllum ruderale & T scap & NAT & NEO & NEO & A, H, S \\
\hline Asteraceae & Tessaria dodonaeifolia & $P$ caesp & NAT & VORAND & NEO & $\mathrm{H}$ \\
\hline Asteraceae & Zexmenia brachylepis & Ch suff & NAT & VORAND & NEO & $\mathrm{H}, \mathrm{S}$ \\
\hline Asteraceae & Aspilia pascalioides & P herb & ENDCS & SURAMA & PAN & $\mathrm{H}$ \\
\hline Asteraceae & Eclipta prostrata & T scap & NAT & PAN & PAN & A, H, S \\
\hline Asteraceae & Pterocaulon purpurascens & Ch herb & NAT & $\mathrm{CHA}$ & PAN & $\mathrm{H}$ \\
\hline Asteraceae & Pterocaulon virgatum & T scap & NAT & DISNEO & PAN & $\mathrm{H}, \mathrm{S}$ \\
\hline Asteraceae & Spilanthes paraguayensis & H rept & END & $\mathrm{CHA}$ & PAN & $\mathrm{H}$ \\
\hline Asteraceae & Wedelia species & Ch herb & NAT & & PAN & $\mathrm{H}$ \\
\hline Asteraceae & Holocheilus hieracioides & Ch herb & ENDCS & DOMCHA & SURAMA & $\mathrm{H}, \mathrm{S}$ \\
\hline Bignoniaceae & Amphilophium cynanchoides & $\mathrm{L}$ frut & NAT & DOMCHA & NEO & A, H, S \\
\hline Bignoniaceae & Dolichandra uncata & L suff/frut & NAT & NEO & NEO & $\mathrm{H}$ \\
\hline Bignoniaceae & Dolichandra unguis-cati & L frut & NAT & NEO & NEO & $\mathrm{H}, \mathrm{S}$ \\
\hline Bignoniaceae & Fridericia dichotoma & L frut & NAT & NEO & NEO & A, H, S \\
\hline Bignoniaceae & Fridericia truncata & L suff/frut & NAT & $\mathrm{CHA}$ & NEO & A, H, S \\
\hline Bignoniaceae & Tabebuia aurea & P scap & NAT & DISNEO & NEO & $\mathrm{H}, \mathrm{S}$ \\
\hline Bignoniaceae & Tabebuia nodosa & P scap & NAT & CHA & NEO & A, H, S \\
\hline Boraginaceae & Heliotropium campestre & H scap & NAT & VORAND & KOS & A, S \\
\hline Boraginaceae & $\begin{array}{l}\text { Heliotropium curassavicum var. } \\
\text { argentinum }\end{array}$ & H rept & NAT & DOMCHA & KOS & $\mathrm{H}, \mathrm{S}$ \\
\hline Boraginaceae & Heliotropium dunaense & H scap/rept & END & CHA & KOS & A, S \\
\hline Boraginaceae & Heliotropium pallescens & Ch suff/herb & ENDCS & CHA & KOS & $\mathrm{H}, \mathrm{S}$ \\
\hline Boraginaceae & Cordia americana & P scap & NAT & DOMNSTW & PAN & $\mathrm{H}, \mathrm{S}$ \\
\hline Boraginaceae & Cordia glabrata & P scap & NAT & DOMNSTW & PAN & $\mathrm{H}, \mathrm{S}$ \\
\hline Boraginaceae & Tournefortia rubicunda & P caesp/herb & NAT & DOMNSTW & PAN & A, H, S \\
\hline Bromeliaceae & Deinacanthon urbanianum & Ch succ & ENDGC & CHA & CHA & A, S \\
\hline Bromeliaceae & $\begin{array}{l}\text { Aechmea distichantha var. } \\
\text { schlumbergeri }\end{array}$ & Ch succ & NAT & DOMNSTW & NEO & $\mathrm{H}, \mathrm{S}$ \\
\hline Bromeliaceae & Bromelia hieronymi & Ch succ & ENDGC & CHA & NEO & A, H, S \\
\hline Bromeliaceae & Bromelia serra & Ch succ & NAT & $\mathrm{CHA}$ & NEO & A, H, S \\
\hline Bromeliaceae & Deuterocohnia carmineoviridiflora & Ch succ & NAT & $\mathrm{CHA}$ & VORAND & $\mathrm{A}, \mathrm{S}$ \\
\hline Bromeliaceae & Dyckia ferox & Ch succ & NAT & SURAMA & NEO & $\mathrm{S}$ \\
\hline Bromeliaceae & Dyckia ragonesei & Ch succ & NAT & CHA & NEO & $\mathrm{S}$ \\
\hline Bromeliaceae & Tillandsia bandensis & E herb & NAT & DOMCHA & NEO & A, H, S \\
\hline Bromeliaceae & Tillandsia duratii var. saxatilis & E succ & NAT & CHA & NEO & A, H, S \\
\hline Bromeliaceae & Tillandsia funebris & E herb & NAT & $\mathrm{CHA}$ & NEO & $A, S$ \\
\hline Bromeliaceae & Tillandsia loliacea & E herb & NAT & SURAMA & NEO & A, H, S \\
\hline Bromeliaceae & Tillandsia mereliana & E succ & END & $\mathrm{CHA}$ & NEO & $\mathrm{S}$ \\
\hline Bromeliaceae & Tillandsia minutiflora & E herb & NAT & VORAND & NEO & A, S \\
\hline Bromeliaceae & Tillandsia recurvifolia & E succ & NAT & CHA & NEO & A, H, S \\
\hline Bromeliaceae & Tillandsia reichenbachii & E succ & NAT & $\mathrm{CHA}$ & NEO & A, $S$ \\
\hline Bromeliaceae & Tillandsia vernicosa & E succ & NAT & $\mathrm{CHA}$ & NEO & $\mathrm{H}, \mathrm{S}$ \\
\hline Cactaceae & Cereus forbesii & P succ & NAT & CHA & AMER & A, H, S \\
\hline Cactaceae & Cereus stenogonus & $\mathrm{P}$ succ & NAT & VORAND & AMER & A, H, S \\
\hline Cactaceae & Opuntia anacantha var. retrorsa & Ch succ & NAT & CHA & AMER & A, S \\
\hline Cactaceae & Opuntia colubrina & Ch succ & NAT & $\mathrm{CHA}$ & AMER & A, S \\
\hline Cactaceae & Opuntia elata var. cardiosperma & $\mathrm{P} / \mathrm{Ch}$ succ & NAT & CHA & AMER & $\mathrm{H}, \mathrm{S}$ \\
\hline Cactaceae & Stetsonia coryne & P succ & NAT & CHA & CHA & A, H, S \\
\hline Cactaceae & Harrisia bonplandii & Ch succ & NAT & CHA & DISNEO & A, H, S \\
\hline Cactaceae & Monvillea cavendischii & Ch succ & NAT & DOMCHA & DISNEO & A, H, S \\
\hline Cactaceae & Monvillea kroenleini & Ch succ & END & $\mathrm{CHA}$ & DISNEO & $\mathrm{S}$ \\
\hline Cactaceae & Monvillea spegazzinii & Ch succ & NAT & $\mathrm{CHA}$ & DISNEO & $\mathrm{S}$ \\
\hline Cactaceae & Quiabentia verticillata & $\mathrm{P}$ succ & NAT & VORAND & DOMNSTW & A, S \\
\hline Cactaceae & Selenicereus setaceus & E succ & NAT & NEO & NEO & $\mathrm{H}$ \\
\hline Cactaceae & Rhipsalis baccifera & E succ & NAT & PAN & PAN & $\mathrm{H}$ \\
\hline Cactaceae & Cleistocactus baumannii & Ch succ & NAT & CHA & VORAND & A, S \\
\hline Cactaceae & Echinopsis rhodotricha & Ch succ & ENDCS & SURAMA & VORAND & $\mathrm{H}, \mathrm{S}$ \\
\hline Cactaceae & Gymnocalycium mihanovichii & Ch succ & ENDGC & CHA & VORAND & A, S \\
\hline Cannabaceae & Celtis ehrenbergiana & P caesp/scap & NAT & NEO & KOS & A, H, S \\
\hline Capparaceae & Sarcotoxicum salicifolium & P caesp & NAT & $\mathrm{CHA}$ & $\mathrm{CHA}$ & A, S \\
\hline
\end{tabular}




\begin{tabular}{|c|c|c|c|c|c|c|}
\hline Familie & Art & LF & STAT & DISSPEC & DISGEN & NG \\
\hline Capparaceae & Capparicordis tweediana & P caesp & NAT & VORAND & DISNEO & A, H, S \\
\hline Capparaceae & Cynophalla retusa & P caesp & NAT & VORAND & NEO & A, H, S \\
\hline Capparaceae & Anisocapparis speciosa & $\mathrm{P}$ caesp & NAT & VORAND & VORAND & A, S \\
\hline Caricaceae & Jacaratia corumbensis & P caesp & ENDGC & CHA & NEO & A, S \\
\hline Caryophyllaceae & Polycarpon suffruticosum & H caesp & NAT & DOMCHA & KOS & A, H, S \\
\hline Caryophyllaceae & Spergula ramosa & $\mathrm{G}$ rad & NAT & VORAND & KOS & $\mathrm{H}, \mathrm{S}$ \\
\hline Celastraceae & Maytenus vitis-idaea & P caesp & NAT & DOMCHA & KOS & $\mathrm{H}, \mathrm{S}$ \\
\hline Celastraceae & Schaefferia argentinensis & P caesp & ENDCS & DOMNSTW & NEO & $\mathrm{H}, \mathrm{S}$ \\
\hline Cleomaceae & Cleome tucumanensis & Ch herb & NAT & $\mathrm{CHA}$ & KOS & A, S \\
\hline Commelinaceae & Commelina erecta & G rhiz & NAT & KOS & KOS & A, S \\
\hline Commelinaceae & Gibasis geniculata & G rhiz & NAT & NEO & NEO & $\mathrm{H}$ \\
\hline Convolvulaceae & Cressa truxillensis & $\mathrm{G}$ rad & NAT & AMPHI & KOS & $\mathrm{H}, \mathrm{S}$ \\
\hline Convolvulaceae & Cuscuta species & TL par & NAT & & KOS & $\mathrm{H}, \mathrm{S}$ \\
\hline Convolvulaceae & Ipomoea amnicola & $\mathrm{L}$ herb & NAT & DISNEO & KOS & A, H, S \\
\hline Convolvulaceae & Ipomoea bonariensis & $L$ herb & NAT & SURAMA & KOS & A, H, S \\
\hline Convolvulaceae & Ipomoea grandifolia & $\mathrm{TL}$ & NAT & SURAMA & KOS & $\mathrm{S}$ \\
\hline Convolvulaceae & Evolvulus convolvuloides & H rept & NAT & DISNEO & NEO & $\mathrm{H}, \mathrm{S}$ \\
\hline Convolvulaceae & Evolvulus sericeus & H rept & NAT & NEO & NEO & A, H, S \\
\hline Convolvulaceae & Jacquemontia lorentzii & $\mathrm{L}$ herb & NAT & SURAMA & PAN & $\mathrm{A}, \mathrm{S}$ \\
\hline Cucurbitaceae & Cucurbitella asperata & $\mathrm{L}$ herb & NAT & VORAND & NEO & A, H, S \\
\hline Сyperaceae & Bulbostylis capillaris & H caesp & NAT & AMER & KOS & $\mathrm{H}, \mathrm{S}$ \\
\hline Сyperaceae & Cyperus aggregatus & G rhiz & NAT & AMER & KOS & $\mathrm{H}, \mathrm{S}$ \\
\hline Сyperaceae & Cyperus corymbosus var. subnodosus & G rhiz & NAT & NEO & KOS & $\mathrm{H}, \mathrm{S}$ \\
\hline Сyperaceae & Cyperus species & G rhiz & NAT & & KOS & $\mathrm{H}, \mathrm{S}$ \\
\hline Сyperaceae & Eleocharis elegans & Hyd rhiz & NAT & NEO & KOS & $\mathrm{H}$ \\
\hline Сyperaceae & Abildgaardia ovata & H caesp & NAT & PAN & PAN & $\mathrm{H}, \mathrm{S}$ \\
\hline Erythroxylaceae & Erythroxylum cuneifolium & P caesp & NAT & DOMNSTW & PAN & $\mathrm{H}, \mathrm{S}$ \\
\hline Euphorbiaceae & Ditaxis montevidensis & Ch suff/P herb & NAT & DOMCHA & AMER & A, H, S \\
\hline Euphorbiaceae & Euphorbia lasiocarpa & H scap & NAT & NEO & KOS & A, S \\
\hline Euphorbiaceae & Euphorbia serpens & T rept & NAT & AMER & KOS & $\mathrm{H}, \mathrm{S}$ \\
\hline Euphorbiaceae & Astraea lobata & Ch suff/herb & NAT & NEO & NEO & A, S \\
\hline Euphorbiaceae & Cnidoscolus albomaculatus & Ch suff/herb & NAT & SURAMA & NEO & A, S \\
\hline Euphorbiaceae & Manihot guaranitica & P caesp/herb & NAT & VORAND & NEO & A, H, S \\
\hline Euphorbiaceae & Microstachys hispida & Ch suff/herb & NAT & DOMNSTW & NEO & $\mathrm{H}, \mathrm{S}$ \\
\hline Euphorbiaceae & Croton bonplandianus & Ch herb & NAT & DOMCHA & PAN & $\mathrm{H}, \mathrm{S}$ \\
\hline Euphorbiaceae & Croton glandulosus & Ch herb & NAT & NEO & PAN & $\mathrm{S}$ \\
\hline Euphorbiaceae & Croton gracilipes & P caesp/herb & NAT & DOMNSTW & PAN & A, H, S \\
\hline Euphorbiaceae & Croton hieronymi & Ch suff/frut & NAT & CHA & PAN & A, H, S \\
\hline Euphorbiaceae & Croton orbignyanus & Ch suff & NAT & VORAND & PAN & $\mathrm{S}$ \\
\hline Euphorbiaceae & Jatropha excisa & P caesp/herb & ENDCS & VORAND & PAN & A, S \\
\hline Euphorbiaceae & Jatropha grossidentata & Ch suff/P herb & NAT & CHA & PAN & A, S \\
\hline Euphorbiaceae & Jatropha hieronymi & P herb/scap & NAT & VORAND & PAN & A, S \\
\hline Euphorbiaceae & Tragia hieronymi & L suff/herb & NAT & CHA & PAN & $\mathrm{S}$ \\
\hline Fabaceae & Chloroleucon tenuiflorum & P scap & NAT & VORAND & AMER & $\mathrm{H}$ \\
\hline Fabaceae & Lophocarpinia aculeatifolia & P caesp & ENDGC & CHA & CHA & $\mathrm{S}$ \\
\hline Fabaceae & Mimozyganthus carinatus & P caesp & NAT & CHA & CHA & $\mathrm{S}$ \\
\hline Fabaceae & Piptadeniopsis lomentifera & $\mathrm{P}$ caesp/scap & ENDGC & CHA & CHA & $\mathrm{S}$ \\
\hline Fabaceae & Senna morongii & P herb & NAT & VORAND & KOS & $\mathrm{H}, \mathrm{S}$ \\
\hline Fabaceae & Senna pendula & P caesp & NAT & NEO & KOS & $\mathrm{H}, \mathrm{S}$ \\
\hline Fabaceae & $\begin{array}{l}\text { Microlobius foetidus subsp. } \\
\text { paraguensis }\end{array}$ & P scap & NAT & CHA & DISNEO & $\mathrm{H}, \mathrm{S}$ \\
\hline Fabaceae & Pterogyne nitens & P scap & NAT & DOMNSTW & DOMNSTW & A, H, S \\
\hline Fabaceae & Centrosema virginianum & $\mathrm{L}$ herb & NAT & NEO & NEO & A, H, S \\
\hline Fabaceae & Cercidium praecox & P caesp & NAT & DISNEO & NEO & A, H, S \\
\hline Fabaceae & Desmanthus virgatus & Ch suff/herb & NAT & DISNEO & NEO & A, H, S \\
\hline Fabaceae & Geoffroea spinosa & P scap & NAT & DOMNSTW & NEO & A, H, S \\
\hline Fabaceae & Macroptilium bracteatum & L suff/herb & NAT & DOMNSTW & NEO & $\mathrm{H}, \mathrm{S}$ \\
\hline Fabaceae & Macroptilium lathyroides & TL & NAT & NEO & NEO & A, H, S \\
\hline Fabaceae & Mimosa debilis var. debilis & Ch suff & NAT & NEO & NEO & $\mathrm{S}$ \\
\hline Fabaceae & Mimosa detinens & P caesp & NAT & CHA & NEO & A, S \\
\hline Fabaceae & Mimosa xanthocentra & Ch suff/herb & NAT & DISNEO & NEO & $\mathrm{A}, \mathrm{S}$ \\
\hline
\end{tabular}




\begin{tabular}{|c|c|c|c|c|c|c|}
\hline Familie & Art & $\mathbf{L F}$ & STAT & DISSPEC & DISGEN & NG \\
\hline Fabaceae & Acacia aroma & P caesp & NAT & VORAND & PAN & A, H, S \\
\hline Fabaceae & Acacia caven & $\mathrm{P}$ caesp/scap & NAT & SURAMA & PAN & $\mathrm{H}$ \\
\hline Fabaceae & Acacia curvifructa & P caesp/scap & ENDGC & CHA & PAN & $\mathrm{H}, \mathrm{S}$ \\
\hline Fabaceae & Acacia praecox & $\mathrm{P}$ caesp/scap & NAT & CHA & PAN & $\mathrm{S}$ \\
\hline Fabaceae & Aeschynomene histrix & Ch herb & NAT & NEO & PAN & $\mathrm{S}$ \\
\hline Fabaceae & Aeschynomene mollicula & CH suff/P herb & NAT & DOMNSTW & PAN & A, H, S \\
\hline Fabaceae & Aeschynomene viscidula & Ch herb & NAT & DISNEO & PAN & A, S \\
\hline Fabaceae & Bauhinia argentinensis & P caesp & ENDGC & $\mathrm{CHA}$ & PAN & A, S \\
\hline Fabaceae & Caesalpinia paraguariensis & P scap & NAT & CHA & PAN & $\mathrm{H}, \mathrm{S}$ \\
\hline Fabaceae & Chamaecrista arachiphylla & Ch suff & ENDGC & CHA & PAN & A, S \\
\hline Fabaceae & Chamaecrista serpens & Ch suff & NAT & NEO & PAN & $\mathrm{S}$ \\
\hline Fabaceae & Desmodium incanum & Ch herb & NAT & NEO & PAN & $\mathrm{H}$ \\
\hline Fabaceae & Galactia latisiliqua & Ch suff/herb & NAT & DISNEO & PAN & A, H, S \\
\hline Fabaceae & Galactia longifolia & Ch suff/herb & NAT & DISNEO & PAN & $\mathrm{H}$ \\
\hline Fabaceae & Neptunia pubescens & H rept & NAT & DISNEO & PAN & $\mathrm{H}, \mathrm{S}$ \\
\hline Fabaceae & Parkinsonia aculeata & $\mathrm{P}$ caesp/scap & NAT & NEO & PAN & $\mathrm{H}, \mathrm{S}$ \\
\hline Fabaceae & Prosopis hassleri & P scap & ENDGC & CHA & PAN & $\mathrm{H}, \mathrm{S}$ \\
\hline Fabaceae & Prosopis kuntzei & P scap & NAT & CHA & PAN & $\mathrm{S}$ \\
\hline Fabaceae & Prosopis nigra & P scap & NAT & DOMCHA & PAN & A, H, S \\
\hline Fabaceae & Prosopis rojasiana & P caesp & END & CHA & PAN & $\mathrm{S}$ \\
\hline Fabaceae & Prosopis ruscifolia & P caesp/scap & NAT & CHA & PAN & $\mathrm{H}, \mathrm{S}$ \\
\hline Fabaceae & Prosopis sericantha & P caesp & NAT & DOMCHA & PAN & A, S \\
\hline Fabaceae & Rhynchosia burkartii & Ch suff & NAT & CHA & PAN & A, S \\
\hline Fabaceae & Sesbania virgata & P caesp & NAT & SURAMA & PAN & $\mathrm{H}$ \\
\hline Fabaceae & Vigna luteola & TL/T rept & NAT & PAN & PAN & $\mathrm{H}, \mathrm{S}$ \\
\hline Fabaceae & Zornia latifolia & H caesp & NAT & PAN & PAN & $\mathrm{H}, \mathrm{S}$ \\
\hline Hydnoraceae & Prosopanche americana & Par/G rhiz & NAT & DOMCHA & NEO & $\mathrm{S}$ \\
\hline Lamiaceae & Salvia cardiophylla & H scap & NAT & CHA & KOS & $\mathrm{H}, \mathrm{S}$ \\
\hline Loranthaceae & Psittacanthus cordatus & HPar & NAT & NEO & NEO & $\mathrm{H}$ \\
\hline Malpighiaceae & Janusia guaranitica & L suff & NAT & SURAMA & NEO & A, S \\
\hline Malpighiaceae & Mascagnia brevifolia & L suff & NAT & VORAND & NEO & A, H, S \\
\hline Malvaceae & Ayenia eliae & Ch suff & NAT & CHA & NEO & $\mathrm{A}, \mathrm{S}$ \\
\hline Malvaceae & Ayenia odonellii & Ch suff & NAT & CHA & NEO & A, H, S \\
\hline Malvaceae & Gaya tarijensis & Ch suff & NAT & VORAND & NEO & A, H, S \\
\hline Malvaceae & Herissantia crispa & Ch suff/herb & NAT & NEO & NEO & $\mathrm{S}$ \\
\hline Malvaceae & Pseudabutilon callimorphum & P herb & NAT & VORAND & NEO & A, H, S \\
\hline Malvaceae & Pseudabutilon virgatum & P caesp/herb & NAT & VORAND & NEO & A, $S$ \\
\hline Malvaceae & Ceiba chodatii & P dol & NAT & VORAND & PAN & A, S \\
\hline Malvaceae & Cienfuegosia argentina & H rept & NAT & CHA & PAN & $\mathrm{S}$ \\
\hline Malvaceae & Cienfuegosia drummondii & H rept & NAT & AMPHI & PAN & $\mathrm{H}, \mathrm{S}$ \\
\hline Malvaceae & Malvastrum coromandelianum & Ch suff & NAT & NEO & PAN & A, H, S \\
\hline Malvaceae & Melochia ministella & Ch suff & ENDGC & CHA & PAN & $\mathrm{H}$ \\
\hline Malvaceae & Melochia parvifolia var. fasciculata & P herb & NAT & DISNEO & PAN & $\mathrm{H}$ \\
\hline Malvaceae & Melochia werdermannii & $\mathrm{P}$ herb & NAT & CHA & PAN & A, S \\
\hline Malvaceae & Pavonia hieronymi & $\mathrm{P}$ herb & NAT & DOMNSTW & PAN & $\mathrm{H}$ \\
\hline Malvaceae & Pavonia vannii & Ch suff & NAT & CHA & PAN & A, S \\
\hline Malvaceae & Sida anomala & H caesp & NAT & VORAND & PAN & $\mathrm{H}, \mathrm{S}$ \\
\hline Malvaceae & Sida cabreriana & $\mathrm{CH}$ suff/P herb & NAT & VORAND & PAN & A, S \\
\hline Malvaceae & Sida dictyocarpa & H scap/rept & NAT & VORAND & PAN & $\mathrm{S}$ \\
\hline Malvaceae & Waltheria communis & H scap & NAT & SURAMA & PAN & $\mathrm{H}, \mathrm{S}$ \\
\hline Malvaceae & Waltheria indica & P herb & NAT & PAN & PAN & A, H, S \\
\hline Malvaceae & Wissadula densiflora & Ch suff & NAT & CHA & PAN & A, H, S \\
\hline Molluginaceae & Glinus radiatus & T rept & NAT & NEO & PAN & A, S \\
\hline Moraceae & Dorstenia brasiliensis & G bulb & NAT & NEO & PAN & $\mathrm{S}$ \\
\hline Nyctaginaceae & Bougainvillea campanulata & $\mathrm{P}$ caesp/scap & ENDGC & CHA & NEO & A, S \\
\hline Nyctaginaceae & Bougainvillea infesta & $\mathrm{P}$ caesp/scap & NAT & $\mathrm{CHA}$ & NEO & A, H, S \\
\hline Nyctaginaceae & Boerhavia diffusa & H rept & NATUR & PAN & PAN & A, H, S \\
\hline Nyctaginaceae & Pisonia hassleriana & P scap & NAT & VORAND & PAN & $\mathrm{H}, \mathrm{S}$ \\
\hline Nyctaginaceae & Pisonia zapallo & P scap & NAT & DOMNSTW & PAN & $\mathrm{H}, \mathrm{S}$ \\
\hline Orchidaceae & Pteroglossa lurida & $\mathrm{G}$ rad & NAT & SURAMA & NEO & $\mathrm{H}, \mathrm{S}$ \\
\hline
\end{tabular}




\begin{tabular}{|c|c|c|c|c|c|c|}
\hline Familie & Art & LF & STAT & DISSPEC & DISGEN & NG \\
\hline Oxalidaceae & Oxalis frutescens & Ch suff & NAT & NEO & KOS & A, S \\
\hline Passifloraceae & Passiflora foetida & L suff & NAT & NEO & PAN & A, H, S \\
\hline Passifloraceae & Passiflora mooreana & L suff & NAT & CHA & PAN & $\mathrm{H}, \mathrm{S}$ \\
\hline Passifloraceae & Turnera grandiflora & H scap & NAT & CHA & PAN & $\mathrm{H}, \mathrm{S}$ \\
\hline Passifloraceae & Turnera krapovickasii & Ch suff & NAT & CHA & PAN & A, S \\
\hline Passifloraceae & Turnera weddelliana & P caesp/herb & NAT & NEO & PAN & $\mathrm{H}, \mathrm{S}$ \\
\hline Phytolaccaceae & Rivina humilis & Ch suff & NAT & NEO & NEO & $\mathrm{H}, \mathrm{S}$ \\
\hline Plantaginaceae & Basistemon spinosus & P caesp & NAT & CHA & DISNEO & $\mathrm{H}, \mathrm{S}$ \\
\hline Plantaginaceae & Scoparia dulcis & Ch suff/herb & NAT & PAN & PAN & A, H, S \\
\hline Plantaginaceae & Stemodia ericifolia & H rept & NAT & CHA & PAN & $\mathrm{H}, \mathrm{S}$ \\
\hline Poaceae & Aristida mendocina & H caesp & NAT & VORAND & KOS & A, S \\
\hline Poaceae & Chloris ciliata & H caesp & NAT & DISNEO & KOS & $\mathrm{H}, \mathrm{S}$ \\
\hline Poaceae & Chloris elata & H caesp & NAT & DISNEO & KOS & A, H, S \\
\hline Poaceae & Coleataenia prionitis & H caesp & NAT & SURAMA & AMER & $\mathrm{H}$ \\
\hline Poaceae & Cynodon dactylon & H rept & NATUR & PAL & PAN & $\mathrm{H}, \mathrm{S}$ \\
\hline Poaceae & Cynodon niemfuensis & H rept & NATUR & PAL & PAN & $\mathrm{S}$ \\
\hline Poaceae & Digitaria insularis & H caesp & NAT & PAN & KOS & $\mathrm{H}, \mathrm{S}$ \\
\hline Poaceae & Digitaria species & H caesp & NAT & & KOS & A, H, S \\
\hline Poaceae & Elionurus muticus & H caesp & NAT & PAN & PAN & $\mathrm{A}, \mathrm{H}, \mathrm{S}$ \\
\hline Poaceae & Eragrostis orthoclada & H caesp & NAT & CHA & KOS & $\mathrm{H}, \mathrm{S}$ \\
\hline Poaceae & Gouinia paraguayensis & H caesp & NAT & CHA & NEO & A, S \\
\hline Poaceae & Lasiacis sorghoidea & P gram & NAT & NEO & NEO & A, H, S \\
\hline Poaceae & Leptochloa fusca subsp. uninervia & $\begin{array}{l}\text { T caesp/ H } \\
\text { caesp }\end{array}$ & NAT & AMPHI & PAN & $\mathrm{H}, \mathrm{S}$ \\
\hline Poaceae & Leptochloa virgata & H caesp & NAT & NEO & PAN & $\mathrm{H}, \mathrm{S}$ \\
\hline Poaceae & Megathyrsus maximus & H caesp & INT & PAL & PAL & $\mathrm{H}, \mathrm{S}$ \\
\hline Poaceae & Pappophorum krapovickasii & H caesp & NAT & DOMNSTW & NEO & A, S \\
\hline Poaceae & Paspalum sp. & H rept & NAT & & PAN & $\mathrm{H}, \mathrm{S}$ \\
\hline Poaceae & Schizachyrium sp. & H rept & NAT & & PAN & $\mathrm{H}, \mathrm{S}$ \\
\hline Poaceae & Setaria geminata & H rept/Hyd rhiz & NAT & AMER & KOS & $\mathrm{H}, \mathrm{S}$ \\
\hline Poaceae & Setaria nicorae & H caesp & NAT & DISNEO & KOS & $\mathrm{H}, \mathrm{S}$ \\
\hline Poaceae & Spartina spartinae & H caesp & NAT & AMPHI & KOS & $\mathrm{S}$ \\
\hline Poaceae & Sporobolus pyramidatus & H caesp & NAT & AMER & KOS & $\mathrm{S}$ \\
\hline Poaceae & Trichloris crinita & H caesp & NAT & AMPHI & AMPHI & $\mathrm{S}$ \\
\hline Poaceae & Urochloa adspersa & T rept & NAT & DISNEO & PAN & A, H, S \\
\hline Poaceae & Urochloa paucispicata & $\mathrm{T}$ rept & NAT & VORAND & PAN & A, H, S \\
\hline Polygonaceae & Coccoloba cordata & $\mathrm{P}$ caesp/scap & NAT & CHA & NEO & $\mathrm{H}, \mathrm{S}$ \\
\hline Polygonaceae & Coccoloba spinescens & $\mathrm{P}$ caesp/scap & NAT & $\mathrm{CHA}$ & NEO & A, H, S \\
\hline Polygonaceae & Salta triflora & $\mathrm{P}$ caesp/scap & NAT & VORAND & VORAND & A, S \\
\hline Polypodiaceae & Microgramma vacciniifolia & E herb & NAT & DOMNSTW & PAN & $\mathrm{H}$ \\
\hline Pontederiaceae & Heteranthera multiflora & Hyd rad & NAT & AMPHI & PAN & $\mathrm{H}, \mathrm{S}$ \\
\hline Portulaccaceae & Portulaca cryptopetala & $\mathrm{T}$ succ/H scap & NAT & DOMCHA & KOS & A, S \\
\hline Portulaccaceae & Portulaca grandiflora & $\mathrm{T}$ succ & ENDCS & DOMCHA & KOS & $\mathrm{S}$ \\
\hline Portulaccaceae & Portulaca oleracea & $\mathrm{T}$ succ & NATUR & KOS & KOS & $\mathrm{H}$ \\
\hline Portulaccaceae & Portulaca sp. & $\mathrm{T}$ succ & NAT & & KOS & A, H, S \\
\hline Pteridaceae & Cheilanthes obducta & G rhiz & NAT & CHA & KOS & $\mathrm{A}, \mathrm{S}$ \\
\hline Rhamnaceae & Ziziphus mistol & P scap & NAT & VORAND & PAN & A, H, S \\
\hline Rubiaceae & Borreria eryngioides & H caesp/scap & NAT & SURAMA & PAN & A, H, S \\
\hline Rubiaceae & Guettarda uruguensis & P caesp & NAT & DOMNSTW & PAN & $\mathrm{H}$ \\
\hline Rubiaceae & Staelia virgata & H caesp & NAT & SURAMA & SURAMA & A, S \\
\hline Ruppiaceae & Ruppia maritima & Hyd rhiz & NAT & KOS & KOS & $\mathrm{H}$ \\
\hline Rutaceae & Zanthoxylum fagara & P scap & NAT & NEO & KOS & $\mathrm{H}$ \\
\hline Santalaceae & Phoradendron argentinum & HPar & NAT & DOMCHA & AMER & A, H, S \\
\hline Santalaceae & Acanthosyris falcata & P scap & NAT & CHA & DISNEO & $\mathrm{S}$ \\
\hline Sapindaceae & Cardiospermum halicacabum & TL & NAT & KOS & KOS & A, H, S \\
\hline Sapindaceae & Serjania marginata & L suff/frut & NAT & SURAMA & NEO & A, H, S \\
\hline Sapindaceae & Serjania sp. & L suff/frut & NAT & & NEO & $\mathrm{S}$ \\
\hline Sapindaceae & Diplokeleba floribunda & P scap & NAT & CHA & VORAND & $\mathrm{H}, \mathrm{S}$ \\
\hline Sapotaceae & Sideroxylon obtusifolium & P scap & NAT & NEO & PAN & A, H, S \\
\hline Selaginellaceae & Selaginella sellowii & H rept & NAT & DISNEO & KOS & A, S \\
\hline Simaroubaceae & Castela coccinea & P caesp & NAT & CHA & DISNEO & $\mathrm{A}, \mathrm{S}$ \\
\hline
\end{tabular}




\begin{tabular}{|c|c|c|c|c|c|c|}
\hline Familie & Art & $\mathbf{L F}$ & STAT & DISSPEC & DISGEN & NG \\
\hline Solanaceae & Lycium americanum & P caesp & NAT & DISNEO & KOS & S \\
\hline Solanaceae & Lycium boerhaviaefolium & P caesp & ENDCS & SURAMA & KOS & $\mathrm{H}, \mathrm{S}$ \\
\hline Solanaceae & Lycium cuneatum & $\mathrm{P}$ caesp & NAT & CHA & KOS & A, S \\
\hline Solanaceae & Lycium morongii & P caesp & NAT & CHA & KOS & $\mathrm{H}, \mathrm{S}$ \\
\hline Solanaceae & Lycium nodosum & P caesp & NAT & $\mathrm{CHA}$ & KOS & $\mathrm{H}, \mathrm{S}$ \\
\hline Solanaceae & Solanum aridum & Ch suff/herb & NAT & CHA & KOS & A, H, S \\
\hline Solanaceae & Solanum elaeagnifolium & Ch herb & NAT & AMPHI & KOS & $\mathrm{S}$ \\
\hline Solanaceae & Solanum glaucophyllum & P caesp & NAT & DOMCHA & KOS & $\mathrm{H}, \mathrm{S}$ \\
\hline Solanaceae & Solanum multispinum & Ch suff/herb & NAT & CHA & KOS & $\mathrm{H}, \mathrm{S}$ \\
\hline Solanaceae & Solanum sisymbriifolium & CH suff/P herb & NAT & NEO & KOS & A, H, S \\
\hline Solanaceae & Schwenckia americana & Ch herb & NAT & NEO & NEO & $\mathrm{H}, \mathrm{S}$ \\
\hline Solanaceae & Capsicum chacoënse & Ch suff & NAT & VORAND & NEO & A, H, S \\
\hline Solanaceae & Sclerophylax spinescens & $\mathrm{T}$ succ & ENDCS & DOMCHA & VORAND & $\mathrm{H}, \mathrm{S}$ \\
\hline Talinaceae & Talinum fruticosum & Ch suff & NATUR & NEO & PAN & A, S \\
\hline Talinaceae & Talinum paniculatum & Ch succ & NATUR & NEO & PAN & $\mathrm{H}, \mathrm{S}$ \\
\hline Talinaceae & Talinum polygaloides & Ch suff & NAT & DOMCHA & PAN & $\mathrm{S}$ \\
\hline Ulmaceae & Phyllostylon rhamnoides & P scap & NAT & DOMNSTW & DOMNSTW & $\mathrm{H}, \mathrm{S}$ \\
\hline Verbenaceae & Aloysia gratissima & P caesp & NAT & AMPHI & AMER & $\mathrm{H}, \mathrm{S}$ \\
\hline Verbenaceae & Aloysia virgata & P caesp & NAT & SURAMA & AMER & A, S \\
\hline Verbenaceae & Lantana balansae & P herb/Ch suff & NAT & DOMCHA & PAN & A, H, S \\
\hline Verbenaceae & Lippia alba & P caesp & NAT & NEO & PAN & $\mathrm{H}, \mathrm{S}$ \\
\hline Verbenaceae & Priva boliviana & H scap & NAT & $\mathrm{CHA}$ & PAN & A, S \\
\hline Vitaceae & Cissus verticillata & $L$ frut & NAT & NEO & KOS & A, H, S \\
\hline Ximeniaceae & Ximenia americana & P scap & NAT & PAN & PAN & A, S \\
\hline Zygophyllaceae & Bulnesia bonariensis & $\mathrm{P}$ caesp/scap & ENDGC & CHA & VORAND & A, S \\
\hline Zygophyllaceae & Bulnesia sarmientoi & P scap & ENDGC & $\mathrm{CHA}$ & VORAND & $\mathrm{S}$ \\
\hline
\end{tabular}


Anhang 4-1: Liste der Probeflächen in den Salzmarschen. Angaben zum Datum der Vegetationsaufnahme, Untersuchungsort, Koordinaten, Höhe über NN (Höhe, m), Gesamtdeckung (Covtotal, \%), Streudeckung (Covlitt, \%), Anteil an offenen Boden (Opensoil, \%), maximale Höhe der Vegetation (Herbmax, m) und die Artenanzahl (Numspec). Die angegebenen Untersuchungsorte sind: Laguna Capitán (LC), Laguna Ganso (LG), Campo León (LE), Campo Maria (CM), Chaco Lodge (CL), Laguna Rojas Silva (RS), Riacho Florida (RF), Riacho Mosquito (RM), Centinella (Cen), Riacho González (RG), Fortín Salazar (FS) und Río Verde (RV).

\begin{tabular}{|c|c|c|c|c|c|c|c|c|c|c|}
\hline Einheit & Probefl. & Datum & Ort & Koordinaten & Höhe & Covtotal & Covlitt & Opensoil & Herbmax & Numspec \\
\hline \multirow{3}{*}{1} & 137 & 15.11 .2012 & $\mathrm{CM}$ & S22 3417.8 W59 2020.2 & 112 & 75 & 0 & 5 & 0 & 1 \\
\hline & 138 & 15.11.2012 & $\mathrm{CM}$ & S22 3420.7 W59 2014.6 & 110 & 40 & 0 & 0 & 0 & 1 \\
\hline & 139 & 15.11.2012 & $\mathrm{CM}$ & S22 34 17.6 W59 2019.1 & 110 & 95 & 0 & 0 & 0.3 & 1 \\
\hline \multirow{10}{*}{2} & 38 & 14.02.2010 & CL & S22 3252.0 W59 1839.3 & 111 & 90 & 30 & 0 & 0.3 & 1 \\
\hline & 39 & 15.02.2010 & CL & S22 3229.9 W59 1753.5 & 106 & 90 & 40 & 0 & 0.3 & 1 \\
\hline & 66 & 06.01 .2011 & RS & S22 3742.7 W59 0342.1 & 105 & 70 & 40 & 10 & 0.5 & 7 \\
\hline & 74 & 19.01.2011 & $\mathrm{LC}$ & S22 3225.9 W59 4035.3 & 122 & 90 & 20 & 10 & 0.4 & 3 \\
\hline & 77 & 19.01.2011 & $\mathrm{LC}$ & S22 3224.9 W59 4035.8 & 122 & 97 & 30 & 0 & 0.5 & 1 \\
\hline & 92 & 23.11.2011 & $\mathrm{RF}$ & S20 4553.1 W59 2107.3 & 110 & 90 & 30 & 15 & 0.3 & 2 \\
\hline & 95 & 24.11.2011 & $\mathrm{RF}$ & S20 4614.0 W59 2114.8 & 109 & 80 & 20 & 25 & 0.3 & 4 \\
\hline & 98 & 25.11.2011 & $\mathrm{RF}$ & S20 4537.0 W59 2055.7 & 110 & 75 & 40 & 20 & 0.3 & 2 \\
\hline & 110 & 17.01.2012 & Cen & S21 5633.8 W59 0502.2 & 109 & 97 & 20 & 0 & 0.6 & 2 \\
\hline & 149 & 25.11.2012 & CL & S22 3247.9 W59 1840.8 & 110 & 95 & 20 & 1 & 0.4 & 1 \\
\hline \multirow{3}{*}{3} & 165 & 22.11.2012 & RG & S22 4243.6 W59 3117.8 & 117 & 98 & 40 & 15 & 0.3 & 1 \\
\hline & 166 & 22.11.2012 & RG & S22 42 47.4 W59 3116.1 & 118 & 80 & 50 & 20 & 0.4 & 1 \\
\hline & 175 & 22.11.2012 & RG & S22 4251.3 W59 1733.1 & 110 & 85 & 25 & 30 & 0.9 & 2 \\
\hline \multirow{12}{*}{4} & 24 & 22.03.2010 & CM & S22 3407.7 W59 2026.0 & 111 & 100 & 10 & 2 & 1.5 & 4 \\
\hline & 34 & 14.02.2010 & CL & S22 3251.1 W59 1840.1 & 111 & 70 & 40 & 0 & 0.3 & 2 \\
\hline & 35 & 15.02.2010 & CL & S22 3230.1 W59 1754.2 & 106 & 85 & 30 & 5 & 0.4 & 4 \\
\hline & 36 & 19.03.2010 & CL & S22 3216.4 W59 1634.5 & 111 & 90 & 40 & 10 & 0.4 & 3 \\
\hline & 37 & 22.03.2010 & $\mathrm{CM}$ & S22 3407.1 W59 2025.3 & 111 & 70 & 50 & 10 & 0.7 & 4 \\
\hline & 111 & 17.01.2012 & Cen & S21 5632.4 W59 0503.5 & 109 & 98 & 20 & 0 & 1 & 2 \\
\hline & 140 & 15.11.2012 & CM & S22 3418.7 W59 2019.0 & 111 & 97 & 15 & 2 & 0.4 & 4 \\
\hline & 142 & 15.11.2012 & $\mathrm{CM}$ & S22 3409.7 W59 2029.6 & 112 & 85 & 40 & 20 & 1 & 3 \\
\hline & 146 & 15.11.2012 & $\mathrm{CM}$ & S22 3412.3 W59 2049.4 & 109 & 75 & 20 & 40 & 0.5 & 3 \\
\hline & 148 & 25.11.2012 & CL & S22 3247.0 W59 1840.2 & 110 & 90 & 30 & 10 & 0.3 & 1 \\
\hline & 153 & 25.11.2012 & CL & S22 3239.6 W59 1853.9 & 111 & 90 & 40 & 0 & 0.4 & 4 \\
\hline & 155 & 25.11.2012 & CL & S22 3237.3 W59 1841.0 & 112 & 85 & 30 & 20 & 0.3 & 3 \\
\hline \multirow{22}{*}{5} & 20 & 14.02 .2010 & CL & S22 3331.8 W59 1841.1 & 108 & 75 & 0 & 30 & 0.6 & 2 \\
\hline & 21 & 14.02.2010 & CL & S22 3254.0 W59 1838.3 & 111 & 70 & 0 & 30 & 0.8 & 1 \\
\hline & 22 & 19.03.2010 & CL & S22 3235.1 W59 1545.5 & 109 & 98 & 10 & 20 & 0.4 & 3 \\
\hline & 23 & 19.03.2010 & CL & S22 3237.2 W59 1845.0 & 108 & 80 & 20 & 20 & 0.5 & 2 \\
\hline & 25 & 19.02.2010 & $\mathrm{CM}$ & S22 3358.5 W59 2130.5 & 110 & 80 & 0 & 15 & 0.5 & 2 \\
\hline & 26 & 18.02.2010 & $\mathrm{CM}$ & S22 3410.0 W59 2028.9 & 112 & 75 & 15 & 15 & 0.5 & 3 \\
\hline & 27 & 14.02.2010 & CL & S22 3333.0 W59 1839.1 & 109 & 70 & 0 & 25 & 0.4 & 3 \\
\hline & 28 & 15.02 .2010 & CL & S22 3236.9 W59 1724.6 & 108 & 40 & 0 & 70 & 0.3 & 3 \\
\hline & 51 & 14.12.2010 & LG & S22 3418.7 W59 3519.0 & 118 & 70 & 0 & 50 & 0.3 & 2 \\
\hline & 62 & 05.01 .2011 & RS & S22 3741.4 W59 0248.1 & 104 & 70 & 5 & 40 & 0.6 & 5 \\
\hline & 68 & 07.01 .2011 & RS & S22 3758.5 W59 0245.6 & 104 & 95 & 0 & 10 & 0.8 & 6 \\
\hline & 75 & 19.01.2011 & $\mathrm{LC}$ & S22 3226.5 W59 4035.0 & 122 & 85 & 0 & 40 & 0.6 & 2 \\
\hline & 80 & 26.01.2011 & LC & S22 3217.3 W59 4037.7 & 121 & 100 & 5 & 10 & 0.8 & 2 \\
\hline & 83 & 26.01.2011 & LE & S22 3455.1 W59 3248.4 & 117 & 100 & 20 & 0 & 0.7 & 2 \\
\hline & 87 & 26.01.2011 & LE & S22 3331.0 W59 2826.9 & 117 & 98 & 10 & 30 & 0.7 & 1 \\
\hline & 88 & 26.01.2011 & LE & S22 3331.1 W59 2830.1 & 117 & 60 & 0 & 80 & 0.4 & 4 \\
\hline & 89 & 26.01.2011 & LE & S22 3332.9 W59 2830.9 & 117 & 98 & 0 & 40 & 0.6 & 2 \\
\hline & 115 & 17.01.2012 & Cen & S22 0218.0 W59 0518.2 & 108 & 50 & 0 & 0 & 0.4 & 2 \\
\hline & 130 & 13.01.2012 & FS & S23 0457.8 W59 1357.1 & 110 & 80 & 0 & 70 & 0.5 & 5 \\
\hline & 131 & 13.01.2012 & FV & S23 1254.7 W59 1209.1 & 109 & 90 & 10 & 40 & 1.2 & 4 \\
\hline & 157 & 25.11.2012 & CL & S22 3243.0 W59 1837.4 & 109 & 70 & 5 & 70 & 0.2 & 2 \\
\hline & 174 & 22.11.2012 & RG & S22 4252.3 W59 1731.5 & 110 & 90 & 30 & 20 & 0.4 & 2 \\
\hline
\end{tabular}


$15 \quad 19.02 .2010 \quad$ CM S22 33 56.9 W59 2118.1

$17 \quad \begin{array}{lll}19.03 .2010 & \text { CL } & \text { S22 } 3216.2 \text { W59 } 1634.4\end{array}$

18 19.03.2010 CL $\quad$ S22 3236.6 W59 1745.2

$19 \quad$ 19.03.2010 $\quad$ CL $\quad$ S22 3238.2 W59 1845.0

$46 \quad$ 08.12.2010 LG S22 3504.6 W59 3513.5

54 14.12.2010 LG S22 3501.1 W59 3534.3

$\begin{array}{llll}56 & \text { 15.12.2010 LG } & \text { S22 } 3440.3 \text { W59 } 3635.2\end{array}$

$\begin{array}{llll}57 & 08.12 .2010 & \text { LG } & \text { S22 } 3459.7 \text { W59 } 3613.8\end{array}$

69 17.01.2011 LC $\quad$ S22 3222.5 W59 4028.5

71 17.01.2011 LC S22 3235.4 W59 4015.4

$\begin{array}{llll}78 & 26.01 .2011 & \text { LC } & \text { S22 } 3237.7 \text { W59 } 4020.4\end{array}$

$90 \quad$ 26.01.2011 LE S22 3330.5 W59 2828.2

94 24.11.2011 RF $\quad$ S20 46 13.2 W59 2115.0

102 08.11.2011 Cen S22 0439.5 W59 0758.0

105 08.11.2011 Cen S22 04 42.4 W59 0749.3

107 06.01.2012 Cen S21 5839.2 W59 0512.5

108 06.01.2012 Cen S21 58 43.0 W59 0511.6

116 04.01.2012 RM S22 1458.0 W59 2757.0

118 04.01.2012 RM S22 14 56.4 W59 2756.4

132 13.01.2012 RV $\quad$ S23 1255.2 W59 1207.0

133 13.01.2012 RV S23 12 56.3 W59 1203.6

136 24.10.2012 RM S22 14 58.2 W59 2756.8

$\begin{array}{llll}161 & 22.11 .2012 & \text { RG } & \text { S22 } 3914.9 \text { W59 } 3649.8\end{array}$

109

110

109

111

108

108

119

115

116

119

120

119

120

117

110

110

110

111

113

119

119

109

109

119

80

20

20

$\begin{array}{lll}80 & 3 & 20\end{array}$

50

50
85

5

20
45

$60 \quad 15$

$60 \quad 15$

85

65

40

50

70

70
40

40
40

98

75

50
60

50

$$
60
$$

60

60
97

70

80

25

90
70

70
30

$120 \quad 60 \quad 15$ $164 \quad 22.11 .2012$ RG $\quad$ S22 4239.5 W59 3116.1

$\begin{array}{rrrrrr}117 & 40 & 5 & 70 & 0.4 & 2 \\ 109 & 75 & 20 & 20 & 1.2 & 2 \\ 110 & 80 & 30 & 25 & 1.5 & 2\end{array}$

$7 \quad-17022.11 .2012$ RG S22 42 50.8 W59 1800.6 $172 \quad 22.11 .2012$ RG $\quad$ S22 42 48.7 W59 $1800.7 \quad 110$ $173 \quad 22.11 .2012$ RG $\quad$ S22 4250.7 W59 1733.6

110

18.02.2010 CM S22 3409.6 W59 2029.1

29 15.02.2010 $\quad$ CL $\quad$ S22 3208.2 W59 1557.4

$\begin{array}{llll}30 & 15.02 .2010 & \text { CL } & \text { S22 } 3216.7 \text { W59 } 1634.4\end{array}$

$\begin{array}{lllll}31 & 15.02 .2010 & \text { CL } & \text { S22 } 3237.7 \text { W59 } 1742.3\end{array}$

32 19.02.2010 CM S22 33 58.9 W59 2131.3

33 19.03.2010 CL S22 32 16.7 W59 1634.8

$40 \quad$ 13.02.2010 $\quad$ CL $\quad$ S22 3259.0 W59 1710.9

41 19.03.2010 CL $\quad$ S22 32 39.6 W59 1841.8

42 22.03.2010 CM S22 34 09.2 W59 2027.3

$43 \quad 22.03 .2010 \quad$ CM $\quad$ S22 3439.8 W59 2247.5

47 14.12.2010 LG $\quad$ S22 3413.5 W59 3511.6

50 14.12.2010 LG S22 34 25.7 W59 3522.6

52 14.12.2010 LG S22 3410.5 W59 3518.0

$\begin{array}{llll}58 & 04.01 .2011 & \text { RS } & \text { S22 } 3739.7 \text { W59 } 0254.5\end{array}$

59 04.01.2011 RS S22 3738.9 W59 0254.3

$\begin{array}{llll}60 & 05.01 .2011 & \text { RS } & \text { S22 } 3742.9 \text { W59 } 0242.6\end{array}$

$\begin{array}{llll}61 & 05.01 .2011 & \text { RS } & \text { S22 } 3742.4 \text { W59 } 0242.7\end{array}$

$63 \quad 05.01 .2011$ RS S22 3741.6 W59 0248.8

$\begin{array}{llll}65 & 06.01 .2011 & \text { RS } & \text { S22 } 3741.5 \text { W59 } 0343.3\end{array}$

$67 \quad 07.01 .2011$ RS S22 3757.8 W59 0257.5

70 17.01.2011 LC S22 3221.5 W59 4028.6

72 17.01.2011 LC $\quad$ S22 3235.6 W59 4015.4

73 17.01.2011 LC S22 32 40.6 W59 4010.7

76 19.01.2011 LC $\quad$ S22 3227.9 W59 4034.8

79 26.01.2011 LC S22 3216.1 W59 4036.3

$81 \quad 26.01 .2011$ LC $\quad$ S22 32 18.4 W59 4037.9

$96 \quad 24.11 .2011$ RF $\quad$ S20 46 14.2 W59 2114.1

99 08.11.2011 Cen S22 0439.0 W59 0808.0

100 08.11.2011 Cen S22 04 39.8 W59 0809.0

109

111

111
107

111

111

109

110

112

111

118

117

117

105

105

104

104

103

106

104

120

119

119

122

121

121

109

111

101 08.11.2011 Cen S22 04 42.7 W59 0804.1

111

112

$80 \quad 30$

25

$\begin{array}{rrr}85 & 40 & 20 \\ 35 & 0 & 60\end{array}$

$40 \quad 0$

$50 \quad 5$

$70 \quad 5$

$80 \quad 0$

$70 \quad 10$

$40 \quad 0$

$85 \quad 20$

$70 \quad 20$

$70 \quad 10$

$60 \quad 10$

$\begin{array}{ll}60 & 10 \\ 60 & 10\end{array}$

$60 \quad 0$

65

95

80

80

50

70

80

0

70

$\begin{array}{ll}1.1 & 1 \\ 0.3 & 3\end{array}$

$0.3 \quad 3$

0.34

$0.3 \quad 3$

$0.3 \quad 1$

0.34

$0.3 \quad 4$

$\begin{array}{ll}0.3 & 4 \\ 0.3 & 2\end{array}$

$\begin{array}{ll}0.5 & 3\end{array}$

$\begin{array}{ll}0.4 & 2 \\ 0.5 & 4\end{array}$

$\begin{array}{ll}0.4 & 4\end{array}$

$0.4 \quad 3$

$\begin{array}{ll}0.4 & 3 \\ 0.6 & 2\end{array}$

$\begin{array}{ll}0.6 & 2 \\ 0.7 & 8\end{array}$




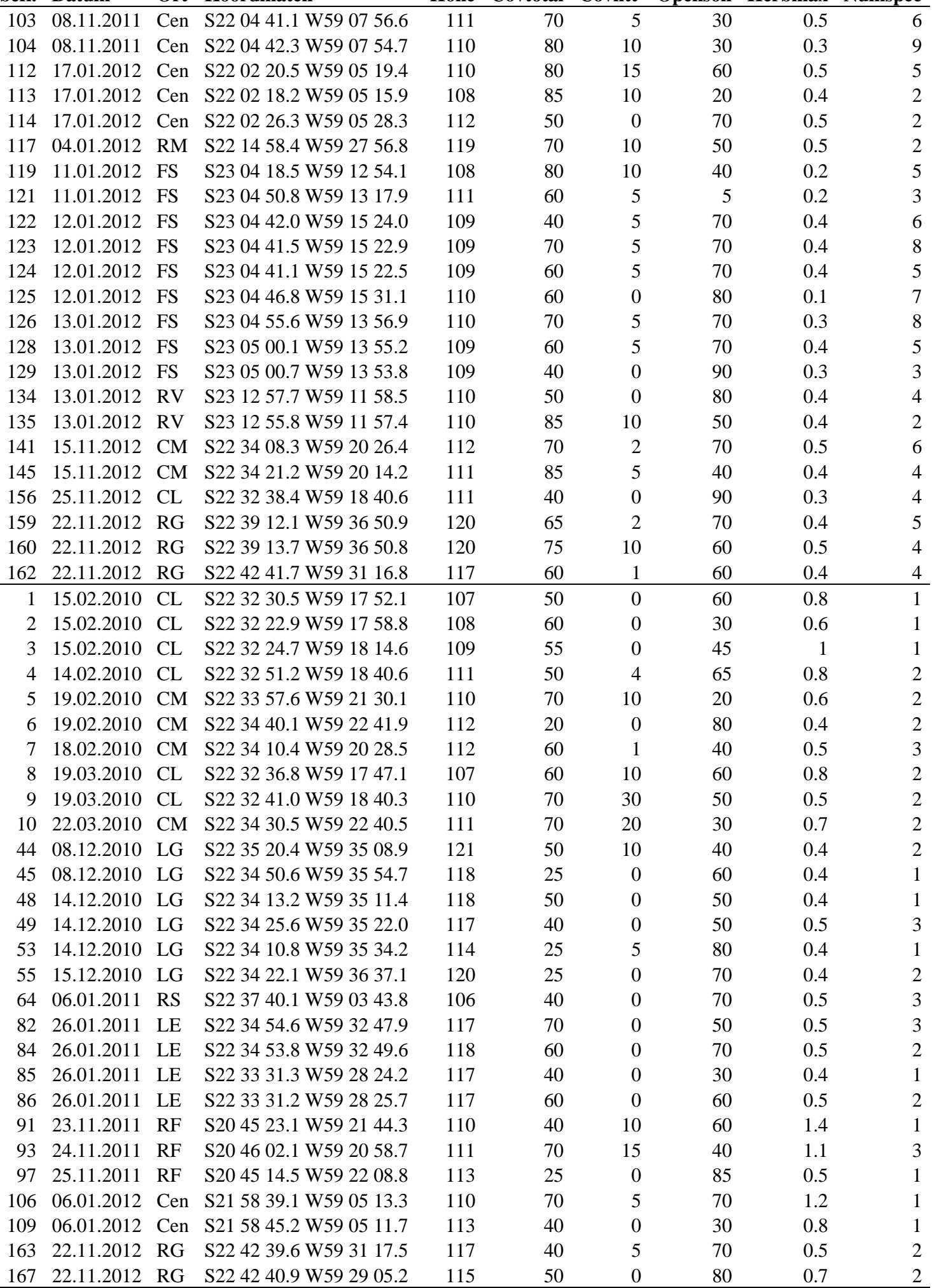


Anhang 4-2: Artenliste der Salzmarschen. Synoptische Tabelle mit Frequenzklassen und Abkürzungen.

\begin{tabular}{|c|c|c|c|c|c|c|c|c|c|c|}
\hline \multirow{2}{*}{ Arten } & \multirow{2}{*}{ Abkürzung } & \multicolumn{9}{|c|}{ Vegetationseinheiten } \\
\hline & & 1 & 2 & 3 & 4 & 5 & 6 & 7 & 8 & 9 \\
\hline Acacia praecox (Jungpflanzen) & ACACPRAE & & & & & I & & & & \\
\hline Alternanthera nodifera & ALTENODI & & & & & & & & & I \\
\hline Atriplex eximia & ATRIEXIM & & & & & & & & I & \\
\hline Coleataenia prionitis & COLEPRIO & & & & & & I & & & \\
\hline Commelina erecta & COMMEREC & & & & & & I & & & \\
\hline Cressa truxillensis & CRESTRUX & & & & I & II & I & & I & \\
\hline Croton bonplandianus & CROTBONP & & & & & & & & I & \\
\hline Cynodon niemfuensis & CYNONIEM & & & & $\mathrm{V}$ & & & & I & \\
\hline Cyperus corymbosus var. subnodosus & CYPECORY & & & $\mathrm{V}$ & & & & & & \\
\hline Echinopsis rhodotricha & ECHIRHOD & & & & & & & & & I \\
\hline Eclipta prostrata & ECLIPROS & & I & & & & & & I & \\
\hline Eleocharis elegans & ELEOELEG & & I & & & & & & & \\
\hline Euphorbia lasiocarpa & EUPHLASI & & I & & & & & & I & \\
\hline Euphorbia serpens & EUPHSERP & & I & & & $\mathrm{I}$ & & & I & \\
\hline Funastrum flavum & FUNAFLAV & & & & & & & & I & \\
\hline Heliotropium curassavicum var. argentinum & HELICURA & & & & I & $\mathrm{I}$ & I & & II & \\
\hline Heteranthera multiflora & HETEMULT & & I & & & & & & & \\
\hline Heterostachys ritteriana & HETERITT & & & & & $\mathrm{I}$ & I & & I & $\mathrm{V}$ \\
\hline Leptochloa fusca subsp. uninervia & LEPTFUUN & & & & III & $\mathrm{V}$ & II & & II & I \\
\hline Leptochloa virgata & LEPTVIRG & & & & I & & & & & \\
\hline Lycium americanum & LYCIAMER & & & & & & & & I & \\
\hline Neptunia pubescens & NEPTPUBE & & I & & & I & & & I & \\
\hline Pappophorum krapovickasii & PAPPKRAP & & & & & & & & I & \\
\hline Parkinsonia aculeata (Jungpflanzen) & PARKACUL & & & & II & & & & I & \\
\hline Paspalum species & PASPSPEC & & I & & & & & & & \\
\hline Pectis odorata & PECTODOR & & & & & & & & I & \\
\hline Portulaca cryptopetala & PORTCRYP & & & & & & I & & I & \\
\hline Portulaca grandiflora & PORTGRAN & & & & & & & & I & I \\
\hline Portulaca oleracea & PORTOLER & & & & & & I & & I & \\
\hline Prosopis ruscifolia (Jungpflanzen) & PROSRUSC & & & & $\mathrm{I}$ & & I & & II & \\
\hline Pterocaulon purpurascens & PTERPURP & & & & & & & & I & \\
\hline Ruellia simplex & RUELSIMP & & I & & & & I & & & \\
\hline Ruppia maritima & RUPPMARI & $\mathrm{V}$ & & & & & & & & \\
\hline Sarcocornia ambigua & SARCAMB & & I & & II & II & $\mathrm{V}$ & IV & II & I \\
\hline Sclerophylax spinescens & SCLESPIN & & & & & & & & I & \\
\hline Sesbania virgata & SESBVIRG & & & & & I & & & & \\
\hline Sesuvium portulacastrum & SESUPORT & & I & II & III & III & III & & $\mathrm{V}$ & I \\
\hline Setaria geminata & SETAGEMI & & $\mathrm{V}$ & & I & & & & & \\
\hline Solanum multispinum & SOLAMULT & & & & & & & & I & \\
\hline Spartina spartinae & SPARSPAR & & & & & & & $\mathrm{V}$ & & \\
\hline Spilanthes paraguayensis & SPILPARA & & I & & & I & & & & \\
\hline Sporobolus pyramidatus & SPORPYRA & & & & & II & II & & IV & I \\
\hline Tessaria dodonaeifolia & TESSDODO & & & & $\mathrm{I}$ & & & & & \\
\hline Trichloris crinita & TRICCRIN & & & & & & I & & I & \\
\hline Urochloa adspersa & UROCADSP & & & & & & & & I & \\
\hline
\end{tabular}




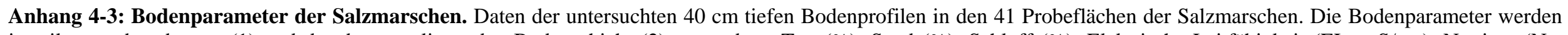

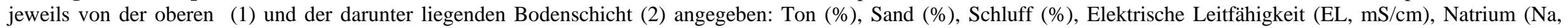
$\mathrm{Cmol}_{\mathrm{c}} / \mathrm{kg}$ ), $\mathrm{pH}$, Organische Substanz (Org, \%), Calcium (Ca, $\mathrm{cmol}_{\mathrm{c}} / \mathrm{kg}$ ) und Magnesium $\left(\mathrm{Mg}, \mathrm{cmol}_{\mathrm{C}} / \mathrm{kg}\right)$.

\begin{tabular}{|c|c|c|c|c|c|c|c|c|c|c|c|c|c|c|c|c|c|c|c|}
\hline Einheit & Probefl. & Ton1 & Ton2 & Sand1 & Sand2 & Schluff1 & Schluff2 & EL1 & EL2 & Na1 & $\mathrm{Na} 2$ & pH1 & pH2 & Org1 & Org2 & Ca1 & $\mathrm{Ca} 2$ & Mg1 & Mg2 \\
\hline 2 & 38 & 25.98 & 28.48 & 51.02 & 49.52 & 23.00 & 22.00 & 7.84 & 8.84 & 8.44 & 9.69 & 7.68 & 8.05 & 0.45 & 0.13 & 33.27 & 43.56 & 5.51 & 6.12 \\
\hline 2 & 66 & 44.00 & 37.40 & 46.00 & 52.80 & 18.00 & 16.20 & 3.16 & 2.96 & 6.57 & 8.92 & 5.11 & 5.39 & 3.15 & 2.64 & 10.61 & 10.03 & 10.06 & 9.97 \\
\hline 2 & 74 & 42.00 & 42.00 & 48.00 & 48.00 & 10.00 & 10.00 & 4.30 & 4.30 & 6.64 & 6.64 & 5.71 & 5.71 & 1.75 & 1.75 & 8.17 & 8.17 & 7.26 & 7.26 \\
\hline 2 & 92 & 40.00 & 40.00 & 16.00 & 16.00 & 4.00 & 4.00 & 4.20 & 4.20 & 6.62 & 6.62 & 4.13 & 4.13 & 5.91 & 5.91 & 4.54 & 4.54 & 1.91 & 1.91 \\
\hline 3 & 4 & 30.88 & 34.48 & 48.22 & 47.52 & 20.90 & 18.00 & 20.11 & 15.35 & 45.39 & 35.94 & 8.41 & 8.66 & 0.44 & 0.29 & 6.12 & 7.52 & 11.37 & 8.94 \\
\hline 3 & 7 & 25.88 & 30.48 & 54.82 & 53.52 & 19.30 & 16.00 & 7.40 & 9.45 & 18.31 & 23.44 & 8.00 & 8.15 & 0.49 & 0.26 & 10.95 & 15.50 & 5.93 & 7.06 \\
\hline 3 & 44 & 24.30 & 30.00 & 66.00 & 60.00 & 9.70 & 10.00 & 8.31 & 8.86 & 31.31 & 33.21 & 8.45 & 8.55 & 0.83 & 0.30 & 21.54 & 27.21 & 9.96 & 9.89 \\
\hline 3 & 53 & 5.05 & 5.00 & 90.05 & 90.00 & 4.90 & 5.00 & 5.81 & 2.19 & 7.11 & 5.69 & 8.44 & 8.46 & 0.13 & 0.12 & 9.02 & 7.37 & 7.92 & 7.82 \\
\hline 3 & 64 & 36.00 & 34.10 & 56.00 & 57.20 & 14.40 & 14.30 & 6.80 & 9.70 & 31.77 & 32.84 & 6.53 & 6.91 & 0.52 & 0.46 & 1.93 & 2.22 & 13.94 & 15.21 \\
\hline 3 & 82 & 5.20 & 24.75 & 91.60 & 68.25 & 3.20 & 8.50 & 5.42 & 6.33 & 28.09 & 31.64 & 6.77 & 8.10 & 0.38 & 0.25 & 5.11 & 4.08 & 8.98 & 8.28 \\
\hline 3 & 85 & 25.85 & 31.00 & 66.85 & 60.00 & 8.90 & 11.00 & 6.58 & 6.70 & 33.95 & 33.95 & 8.21 & 8.58 & 0.75 & 0.33 & 7.61 & 5.11 & 9.98 & 9.98 \\
\hline 3 & 91 & 28.75 & 41.00 & 39.85 & 15.00 & 31.40 & 44.00 & 15.94 & 15.20 & 32.61 & 31.48 & 6.67 & 7.05 & 1.19 & 0.51 & 0.65 & 0.79 & 3.56 & 3.64 \\
\hline 3 & 106 & 27.75 & 30.00 & 61.85 & 61.00 & 10.40 & 9.00 & 19.38 & 14.40 & 106.21 & 33.09 & 7.36 & 6.96 & 0.62 & 0.32 & 6.17 & 3.75 & 9.26 & 8.31 \\
\hline 6 & 34 & 31.98 & 36.48 & 45.02 & 41.52 & 23.00 & 22.00 & 11.25 & 5.00 & 6.37 & 6.09 & 6.23 & 6.67 & 1.67 & 0.71 & 2.48 & 2.38 & 4.00 & 4.14 \\
\hline 7 & 14 & 47.28 & 46.48 & 44.32 & 43.52 & 8.40 & 10.00 & 9.15 & 9.76 & 27.81 & 32.81 & 5.66 & 6.31 & 2.27 & 1.09 & 2.77 & 2.77 & 11.10 & 11.29 \\
\hline 7 & 46 & 37.20 & 42.00 & 52.80 & 47.25 & 10.00 & 10.00 & 4.71 & 5.77 & 31.47 & 31.43 & 7.78 & 8.20 & 0.60 & 0.14 & 6.80 & 14.93 & 9.71 & 9.95 \\
\hline 7 & 69 & 5.00 & 00 & 90.25 & 90.00 & 4.75 & 5.00 & 6.05 & 5.80 & 29.71 & 29.32 & 6.62 & .77 & .80 & 0.52 & 4.34 & 4.08 & 6.69 & 6.35 \\
\hline 7 & 71 & 8.20 & 11.00 & 85.80 & 81.00 & 6.00 & 8.00 & 5.94 & 5.90 & 28.09 & 27.78 & 8.35 & 8.65 & 0.35 & 0.17 & 3.06 & 3.06 & 6.26 & 6.35 \\
\hline 7 & 94 & 8.15 & 5.00 & 28.10 & 5.00 & 63.75 & 90.00 & 10.06 & 11.00 & 18.72 & 20.98 & 6.69 & 7.17 & 0.68 & 0.23 & 1.52 & 1.38 & 1.90 & 1.91 \\
\hline 7 & 107 & 40.00 & 40.00 & 34.75 & 16.00 & 25.25 & 44.00 & 8.10 & 6.90 & 34.50 & 33.90 & 6.84 & 7.00 & 1.82 & 1.15 & 7.45 & 6.71 & 8.84 & 8.31 \\
\hline 7 & 116 & 40.00 & 40.00 & 41.00 & 41.00 & 19.00 & 19.00 & 17.10 & 17.10 & 33.90 & 33.90 & 7.74 & 7.74 & 0.91 & 0.91 & 11.65 & 11.65 & 9.24 & 9.24 \\
\hline 7 & 132 & 13.60 & 40.00 & 77.20 & 42.00 & 7.60 & 18.00 & 5.76 & 10.40 & 30.18 & 34.70 & 7.95 & 7.49 & 0.57 & 0.81 & 6.12 & 11.65 & 8.12 & 10.18 \\
\hline 8 & 21 & 35.28 & 36.48 & 44.52 & 41.52 & 20.80 & 22.00 & 7.31 & 6.62 & 14.10 & 13.13 & 7.58 & 7.66 & 1.20 & 0.74 & 8.32 & 6.34 & 8.57 & 8.47 \\
\hline 8 & 26 & 22.88 & 26.48 & 52.72 & 51.52 & 24.40 & 22.00 & 3.63 & 5.00 & 4.16 & 4.53 & 7.50 & 7.64 & 1.65 & 1.09 & 3.40 & 2.77 & 3.71 & 3.86 \\
\hline 8 & 27 & 30.98 & 20.88 & 52.02 & 59.72 & 18.00 & 22.40 & 6.82 & 6.67 & 3.07 & 15.84 & 8.06 & 8.51 & 4.49 & 0.58 & 19.38 & 11.72 & 11.09 & 8.32 \\
\hline 8 & 51 & 22.00 & 16.00 & 66.00 & 70.00 & 12.80 & 14.00 & 1.21 & 1.49 & 19.61 & 22.14 & 8.05 & 8.42 & 0.89 & 0.15 & 14.74 & 6.42 & 7.78 & 6.67 \\
\hline 8 & 83 & 31.00 & 31.00 & 60.00 & 60.00 & 9.00 & 9.00 & 8.30 & 8.30 & 14.66 & 14.66 & 8.31 & 8.31 & 0.71 & 0.71 & 6.13 & 6.13 & 9.07 & 9.07 \\
\hline
\end{tabular}




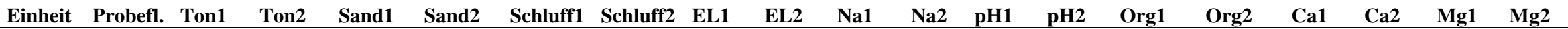

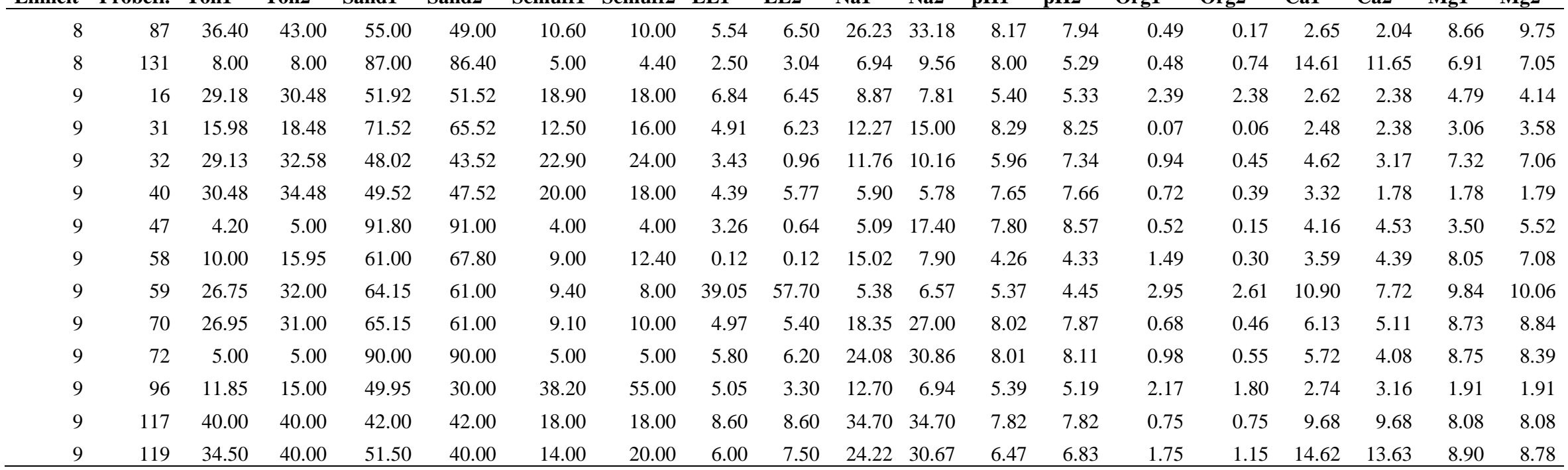




\section{Ordinationen}

Anhang 4-4: Log-file der Korrespondenzanalyse (DCA) zur Ermittlung der Gradientenlänge

\begin{tabular}{|c|c|c|c|c|c|}
\hline Axes & 2 & 3 & 4 & \multicolumn{2}{|c|}{ Total inertia } \\
\hline Eigenvalues & 0.872 & 0.477 & 0.317 & 0.203 & 5.119 \\
\hline Lengths of gradient & 5.369 & 3.460 & 3.255 & 2.617 & \\
\hline Species-environment correlations & : & 0.640 & 0.546 & 0.409 & 0.361 \\
\hline Cumulative percentage variance & & & & & \\
\hline of species data & 17.0 & 26.4 & 32.6 & 36.5 & \\
\hline of species-environment relation: & 30.9 & 44.6 & 0.0 & 0.0 & \\
\hline
\end{tabular}

Sum of all eigenvalues $\quad 5.119$

$\begin{array}{lr}\text { Sum of all canonical eigenvalues } & 1.030\end{array}$

Anhang 4-5: Log-file der Hauptgradientenanalyse (PCA) des Leptochloa-Sesuvium-Sarcocornia Komplexes

\begin{tabular}{|c|c|c|c|c|c|}
\hline Axes & 2 & 3 & 4 & \multicolumn{2}{|c|}{ Total variance } \\
\hline Eigenvalues & 0.314 & 0.263 & 0.147 & 0.067 & 1.000 \\
\hline Species-environment correlations & : & 0.558 & 0.533 & 0.471 & 0.412 \\
\hline \multicolumn{6}{|l|}{ Cumulative percentage variance } \\
\hline of species data $\quad:$ & 31.4 & 57.7 & 72.3 & 79.1 & \\
\hline of species-environment relation: & 38.9 & 68.7 & 81.6 & 86.1 & \\
\hline
\end{tabular}

$\begin{array}{ll}\text { Sum of all } \quad \text { eigenvalues } & 1.000 \\ \text { Sum of all canonical } \quad \text { eigenvalues } & 0.251\end{array}$

Anhang 4-6: Log-file der indirekten Gradientenanalyse (DCA) der Bodenparameter

\begin{tabular}{|c|c|c|c|c|c|}
\hline Axes & 2 & 3 & 4 & \multicolumn{2}{|c|}{ Total inertia } \\
\hline Eigenvalues & 0.957 & 0.562 & 0.326 & 0.152 & 5.280 \\
\hline Lengths of gradient & 7.743 & 4.201 & 2.980 & 1.680 & \\
\hline Species-environment correlations & : & 0.491 & 0.445 & 0.403 & 0.388 \\
\hline Cumulative percentage variance & & & & & \\
\hline of species data & 18.1 & 28.8 & 34.9 & 37.8 & \\
\hline of species-environment relation: & 22.2 & 35.0 & 0.0 & 0.0 & \\
\hline
\end{tabular}

Sum of all eigenvalues $\quad 5.280$

$\begin{array}{ll}\text { Sum of all canonical eigenvalues } & 0.926\end{array}$

Anhang 4-7: Log-file der direkten Gradientenanalyse (CCA) der Bodenparameter.

Analyse der 41 Probeflächen mit Bodenproben zur Ermittlung der signifikanten Bodenvariablen

No. of active species: 30

\begin{tabular}{|c|c|c|c|c|c|c|}
\hline Axes & 2 & 3 & 4 & \multicolumn{3}{|c|}{ Total inertia } \\
\hline Eigenvalues & 0.459 & 0.367 & 0.157 & 0.797 & & \\
\hline Species-environment correlations & 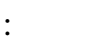 & 0.743 & 0.807 & 0.519 & 0.000 & \\
\hline \multicolumn{7}{|l|}{ Cumulative percentage variance } \\
\hline of species data & 8.7 & 15.6 & 18.6 & 33.7 & & \\
\hline of species-environment relation: & 46.7 & 84.1 & 100.0 & 0.0 & & \\
\hline eigenvalues & & & & & & 5.280 \\
\hline Sum of all canonical eigenvalue & & & & & & 0.983 \\
\hline
\end{tabular}


**** Start of forward selection of variables ****

*** Unrestricted permutation ***

Seeds: 23239945

$\underline{\text { Manuelle Auswahl der Umweltvariablen mit einer Signifikanz } \leq 0.05}$

Org2: P-value 0.0100 (variable 14; F-ratio= 3.09; number of permutations= 499)

Org1: P-value 0.0300 (variable 13; F-ratio= 3.00; number of permutations $=499$ )

Na1: P-value 0.0460 (variable 9 ; F-ratio $=2.04$; number of permutations $=499$ ) 
Anhang 5-1: Liste der Probeflächen in der Gehölzvegetation. Angaben zum Datum der Vegetationsaufnahme, Untersuchungsort, Koordinaten, Höhe über NN (Höhe, m), Deckung (Total, Baumschicht, Strauchschicht, Krautschicht, \%), Höhe der Vegetation (Baumschicht, Strauchschicht, Krautschicht, m) und die Artenanzahl (Art-Nr). Die angegebenen Untersuchungsorte sind: Riacho Florida (RF), Yaguareté Porá (YP), Centinella (Cen), Riacho Mosquito (RM), Laguna Capitán (LC), Laguna Ganso (LG), Campo León (LE), Campo Maria (CM), Chaco Lodge (CL), Laguna Rojas Silva (RS), Riacho González (RG), Fortín Salazar (FS) und Río Verde (RV).

\begin{tabular}{|c|c|c|c|c|c|c|c|c|c|c|c|c|c|}
\hline \multirow{2}{*}{$\begin{array}{l}\text { Ein } \\
\text { heit }\end{array}$} & \multirow{2}{*}{$\begin{array}{l}\text { Prob. } \\
\text { fl. }\end{array}$} & \multirow[t]{2}{*}{ Datum } & \multirow[t]{2}{*}{ Ort } & \multirow[t]{2}{*}{ Koordinaten } & \multirow{2}{*}{$\begin{array}{l}\text { Höhe } \\
\text { NN }\end{array}$} & \multicolumn{4}{|c|}{ Deckung - Vegetation } & \multicolumn{3}{|c|}{ Höhe - Vegetation } & \multirow{2}{*}{$\begin{array}{l}\text { Art- } \\
\mathrm{Nr}\end{array}$} \\
\hline & & & & & & Total & Baum & Strauch & Kraut & Baum & Strauch & Kraut & \\
\hline 10 & 94 & 04.01 .2011 & RS & S22 3736.7 W59 0254.5 & 104 & 85 & & 60 & 75 & & 3.5 & 1.2 & 11 \\
\hline 10 & 104 & 07.01 .2011 & RS & S22 3758.8 W59 0244.2 & 104 & 70 & & 60 & 60 & & 4.5 & 1.5 & 17 \\
\hline 10 & 105 & 07.01 .2011 & RS & S22 3747.1 W59 0238.6 & 112 & 60 & 4 & 60 & 50 & 6 & 3.5 & 1.5 & 11 \\
\hline 10 & 163 & 25.11.2011 & $\mathrm{RF}$ & S20 4446.7 W59 1808.1 & 112 & 70 & & 60 & 30 & & 4 & 1.5 & 16 \\
\hline 10 & 166 & 26.11.2011 & $\mathrm{RF}$ & S20 4530.9 W59 1848.2 & 117 & 85 & 5 & 40 & 50 & 5 & 4 & 1.5 & 7 \\
\hline 10 & 167 & 26.11.2011 & $\mathrm{RF}$ & S20 4527.5 W59 1848.7 & 110 & 50 & 30 & 10 & 20 & 5.5 & 3.5 & 1.3 & 13 \\
\hline 10 & 208 & 11.01.2012 & FS & S23 0436.5 W59 1243.2 & 112 & 80 & & 60 & 40 & & 4.5 & 0.8 & 13 \\
\hline 10 & 225 & 15.11 .2012 & $\mathrm{CM}$ & S22 34 17.7 W59 2020.5 & 112 & 50 & & 40 & 10 & & 3.5 & 0.4 & 6 \\
\hline 10 & 226 & 15.11.2012 & $\mathrm{CM}$ & S22 3420.9 W59 2013.7 & 121 & 70 & & 60 & 15 & & 3 & 0.4 & 6 \\
\hline 11 & 95 & 05.01 .2011 & RS & S22 3742.1 W59 0243.4 & 99 & 95 & 6 & 85 & 50 & 4.5 & 3.5 & 1 & 12 \\
\hline 11 & 96 & 05.01 .2011 & RS & S22 3740.8 W59 0243.8 & 106 & 70 & & 70 & 15 & & 4 & 1 & 8 \\
\hline 11 & 102 & 06.01 .2011 & RS & S22 3740.8 W59 0343.7 & 108 & 90 & & 90 & 10 & & 4 & 1 & 9 \\
\hline 11 & 106 & 07.01 .2011 & RS & S22 3747.2 W59 0238.1 & 107 & 80 & 25 & 20 & 60 & 7 & 3.5 & 1.3 & 18 \\
\hline 11 & 140 & 23.11.2011 & $\mathrm{RF}$ & S20 4522.6 W59 2146.1 & 108 & 70 & & 25 & 50 & & 3.5 & 0.6 & 9 \\
\hline 11 & 143 & 23.11.2011 & $\mathrm{RF}$ & S20 4553.3 W59 2105.5 & 116 & 70 & & 60 & 40 & & 3 & 0.5 & 15 \\
\hline 11 & 144 & 23.11.2011 & $\mathrm{RF}$ & S20 4553.1 W59 2106.7 & 119 & 75 & & 50 & 70 & & 3.5 & 0.7 & 9 \\
\hline 11 & 161 & 25.11.2011 & $\mathrm{RF}$ & S20 4537.7 W59 2056.6 & 103 & 70 & 4 & 40 & 30 & 5 & 4 & 0.5 & 12 \\
\hline 11 & 162 & 25.11.2011 & $\mathrm{RF}$ & S20 4536.4 W59 2056.8 & 111 & 80 & & 70 & 50 & & 4 & 1 & 6 \\
\hline 11 & 165 & 25.11.2011 & $\mathrm{RF}$ & S20 4442.0 W59 1812.2 & 104 & 85 & 10 & 50 & 10 & 5 & 4 & 0.6 & 15 \\
\hline 11 & 190 & 08.11 .2011 & Cen & S22 0444.9 W59 0751.2 & 108 & 85 & & 70 & 80 & & 5 & 0.6 & 8 \\
\hline 11 & 209 & 11.01.2012 & FS & S23 0434.8 W59 1242.5 & 120 & 60 & 5 & 5 & 40 & 6 & 3.5 & 1 & 27 \\
\hline 11 & 213 & 12.01 .2012 & FS & S23 0445.5 W59 1528.1 & 108 & 80 & & 60 & 75 & & 3 & 1.5 & 22 \\
\hline 11 & 231 & 25.11 .2012 & CL & S22 3208.5 W59 1845.2 & 115 & 50 & 40 & 2 & 10 & 5 & 2 & 0.6 & 7 \\
\hline 12 & 1 & 11.02 .2010 & CL & S22 3259.4 W59 1711.0 & 103 & 70 & 25 & 50 & 70 & 5 & 3 & 0.5 & 14 \\
\hline 12 & 2 & 11.02 .2010 & CL & S22 3259.7 W59 1710.7 & 120 & 75 & 15 & 65 & 70 & 5 & 3 & 0.6 & 17 \\
\hline 12 & 168 & 08.12 .2011 & YP & S21 4126.4 W58 4052.0 & 106 & 92 & & 50 & 90 & & 4 & 1.2 & 23 \\
\hline 12 & 169 & 08.12 .2011 & YP & S21 4126.0 W58 4048.4 & 107 & 85 & & 70 & 50 & & 4 & 1.2 & 19 \\
\hline 12 & 170 & 08.12 .2011 & YP & S21 4123.9 W58 4046.9 & 96 & 80 & & 60 & 50 & & 3.5 & 1.2 & 19 \\
\hline 12 & 171 & 08.12 .2011 & YP & S21 4128.7 W58 4053.3 & 107 & 90 & 30 & 50 & 60 & 5 & 3.5 & 1.3 & 18 \\
\hline 12 & 172 & 08.12 .2011 & YP & S21 4129.5 W58 4054.5 & 103 & 95 & 60 & 15 & 85 & 6 & 2.2 & 1.2 & 14 \\
\hline 12 & 189 & 08.11 .2011 & Cen & S22 0442.1 W59 0751.1 & 102 & 70 & & 30 & 40 & & 3 & 0.8 & 8 \\
\hline 12 & 203 & 10.01 .2012 & FS & S23 0452.5 W59 1701.3 & 108 & 80 & 60 & 15 & 40 & 5 & 3.5 & 1 & 20 \\
\hline 12 & 205 & 10.01 .2012 & FS & S23 0453.4 W59 1704.1 & 109 & 60 & & 50 & 60 & & 3 & 1 & 16 \\
\hline 12 & 206 & 10.01.2012 & FS & S23 0455.3 W59 1728.2 & 111 & 85 & 50 & 40 & 60 & 7 & 4 & 1 & 29 \\
\hline 12 & 232 & 25.11 .2012 & CL & S22 32 07.1 W59 1846.8 & 124 & 75 & 40 & 50 & 20 & 6.5 & 3.5 & 0.7 & 12 \\
\hline 13 & 23 & 15.02 .2010 & CL & S22 3223.0 W59 1749.8 & 123 & 75 & 30 & 35 & 70 & 4.5 & 3 & 1 & 18 \\
\hline 13 & 24 & 15.02 .2010 & CL & S22 3214.9 W59 1633.5 & 117 & 90 & & 80 & 5 & & 3.5 & 0.8 & 7 \\
\hline 13 & 65 & 08.12 .2010 & LG & S22 3449.4 W59 3618.2 & 120 & 85 & & 70 & 15 & & 3 & 1 & 11 \\
\hline 13 & 66 & 08.12 .2010 & LG & S22 3504.1 W59 3513.1 & 127 & 95 & 3 & 70 & 20 & 5.5 & 3.5 & 1 & 16 \\
\hline 13 & 92 & 15.12 .2010 & LG & S22 3436.8 W59 3643.1 & 116 & 95 & & 80 & 10 & & 4 & 1 & 12 \\
\hline 13 & 97 & 05.01 .2011 & RS & S22 3740.3 W59 0244.6 & 106 & 97 & & 90 & 15 & & 4.5 & 1 & 13 \\
\hline 13 & 116 & 17.01.2011 & LC & S22 3235.4 W59 4015.0 & 124 & 85 & & 80 & 30 & & 3.5 & 0.8 & 15 \\
\hline 13 & 117 & 17.01.2011 & LC & S22 3243.2 W59 4012.4 & 119 & 85 & & 75 & 40 & & 4 & 1 & 11 \\
\hline 13 & 122 & 17.01.2011 & LC & S22 3241.3 W59 4009.6 & 128 & 85 & & 75 & 20 & & 4.5 & 0.7 & 8 \\
\hline 13 & 124 & 19.01.2011 & LC & S22 3223.7 W59 4017.2 & 121 & 95 & 20 & 85 & 15 & 6 & 3.5 & 0.8 & 14 \\
\hline 13 & 157 & 24.11.2011 & $\mathrm{RF}$ & S20 4614.8 W59 2113.7 & 96 & 80 & & 75 & 50 & & 4 & 1 & 16 \\
\hline 13 & 158 & 25.11 .2011 & $\mathrm{RF}$ & S20 45 14.7 W59 2207.3 & 100 & 50 & & 50 & 20 & & 2.7 & 1.2 & 16 \\
\hline 13 & 186 & 08.11 .2011 & Cen & S22 0441.7 W59 0757.1 & 101 & 97 & & 70 & 40 & & 3.5 & 1.4 & 13 \\
\hline 13 & 201 & 04.01 .2012 & $\mathrm{RM}$ & S22 1459.5 W59 2757.7 & 127 & 80 & & 70 & 5 & & 3.5 & 0.5 & 8 \\
\hline 13 & 214 & 12.01 .2012 & FS & S23 0446.2 W59 1531.4 & 118 & 80 & & 80 & 70 & & 4.5 & 1.5 & 17 \\
\hline
\end{tabular}




\begin{tabular}{|c|c|c|c|c|c|c|c|c|c|c|c|c|c|}
\hline 13 & 229 & 25.11 .2012 & CL & S22 3236.2 W59 1851.6 & 113 & 95 & & 75 & 40 & & 3.5 & 0.7 & 5 \\
\hline 13 & 235 & 22.11.2012 & RG & S22 3915.2 W59 3650.2 & 117 & 95 & & 95 & 40 & & 4 & 1.3 & 20 \\
\hline 13 & 236 & 22.11.2012 & RG & S22 4243.3 W59 2907.2 & 113 & 85 & 20 & 40 & 80 & 5 & 3.5 & 1 & 11 \\
\hline 13 & 238 & 22.11.2012 & RG & S22 4241.6 W59 2749.1 & 118 & 70 & & 60 & 30 & & 3.5 & 0.7 & 14 \\
\hline 14 & 11 & 16.02 .2010 & $\mathrm{CM}$ & S22 3410.6 W59 2028.3 & 116 & 85 & 30 & 70 & 30 & 5.5 & 4 & 1 & 25 \\
\hline 14 & 20 & 13.02.2010 & CL & S22 3254.2 W59 1840.5 & 108 & 85 & & 70 & 40 & & 3.5 & 1 & 22 \\
\hline 14 & 21 & 14.02 .2010 & CL & S22 3221.8 W59 1808.8 & 95 & 90 & & 75 & 30 & & 3 & 1 & 8 \\
\hline 14 & 22 & 15.02 .2010 & CL & S22 3224.0 W59 1750.2 & 114 & 95 & & 90 & 10 & & 3 & 1 & 12 \\
\hline 14 & 26 & 19.03.2010 & CL & S22 3225.0 W59 1754.6 & 119 & 90 & & 80 & 30 & & 3.5 & 1 & 17 \\
\hline 14 & 27 & 19.02.2010 & $\mathrm{CM}$ & S22 3440.6 W59 2242.3 & 121 & 90 & & 75 & 30 & & 3 & 1 & 23 \\
\hline 14 & 29 & 20.02.2010 & $\mathrm{CM}$ & S22 3427.7 W59 2311.8 & 94 & 98 & & 95 & 30 & & 3 & 1 & 28 \\
\hline 14 & 30 & 20.02.2010 & $\mathrm{CM}$ & S22 3438.0 W59 2252.8 & 113 & 95 & & 90 & 20 & & 4 & 1 & 19 \\
\hline 14 & 31 & 20.02 .2010 & $\mathrm{CM}$ & S22 3414.3 W59 2054.8 & 117 & 90 & & 80 & 30 & & 4.5 & 1 & 16 \\
\hline 14 & 32 & 22.03.2010 & $\mathrm{CM}$ & S22 3430.1 W59 2240.2 & 118 & 95 & & 90 & 30 & & 4 & 1 & 21 \\
\hline 14 & 62 & 08.12 .2010 & LG & S22 3520.2 W59 3508.9 & 116 & 85 & 10 & 50 & 25 & 5 & 4 & 1 & 21 \\
\hline 14 & 68 & 10.12.2010 & LG & S22 3415.3 W59 3545.9 & 98 & 97 & & 70 & 30 & & 4 & 0.8 & 18 \\
\hline 14 & 79 & 14.12.2010 & LG & S22 34 13.4 W59 3509.1 & 115 & 95 & & 85 & 3 & & 3 & 1 & 13 \\
\hline 14 & 84 & 14.12 .2010 & LG & S22 3434.7 W59 3537.8 & 115 & 95 & & 85 & 20 & & 4 & 1.5 & 22 \\
\hline 14 & 85 & 15.12 .2010 & LG & S22 3428.8 W59 3629.1 & 121 & 97 & & 95 & 20 & & 4 & 1 & 26 \\
\hline 14 & 91 & 15.12.2010 & LG & S22 3422.2 W59 3638.6 & 116 & 90 & & 90 & 70 & & 3.5 & 1 & 15 \\
\hline 14 & 119 & 17.01.2011 & $\mathrm{LC}$ & S22 3222.0 W59 4019.9 & 115 & 95 & 20 & 80 & 40 & 5.5 & 3 & 1 & 19 \\
\hline 14 & 137 & 26.01.2011 & LE & S22 3331.6 W59 2823.7 & 111 & 100 & & 90 & 40 & & 4 & 1.2 & 20 \\
\hline 14 & 139 & 26.01.2011 & LE & S22 3334.2 W59 2826.9 & 116 & 95 & & 90 & 40 & & 4.5 & 1 & 21 \\
\hline 14 & 159 & 25.11.2011 & $\mathrm{RF}$ & S20 4514.1 W59 2209.5 & 106 & 95 & & 90 & 50 & & 3 & 1.2 & 18 \\
\hline 14 & 227 & 15.11.2012 & $\mathrm{CM}$ & S22 3418.8 W59 2021.8 & 114 & 85 & & 85 & 10 & & 3 & 1 & 12 \\
\hline 14 & 237 & 22.11.2012 & RG & S22 4241.6 W59 2905.4 & 118 & 92 & & 75 & 40 & & 3 & 1 & 15 \\
\hline 14 & 239 & 22.11.2012 & RG & S22 4242.2 W59 2746.7 & 125 & 97 & & 80 & 70 & & 4 & 1.2 & 27 \\
\hline 15 & 4 & 12.02 .2010 & CL & S22 3300.2 W59 1710.6 & 130 & 65 & 25 & 30 & 50 & 7 & 3 & 1.2 & 20 \\
\hline 15 & 5 & 12.02.2010 & CL & S22 3259.9 W59 1709.9 & 106 & 75 & 45 & 25 & 60 & 6 & 2.5 & 1.5 & 17 \\
\hline 15 & 6 & 12.02.2010 & CL & S22 3300.6 W59 1709.6 & 111 & 90 & 30 & 30 & 70 & 6.5 & 3 & 1.5 & 20 \\
\hline 15 & 7 & 12.02 .2010 & CL & S22 3300.6 W59 1709.4 & 113 & 70 & 30 & 40 & 50 & 7 & 3 & 1 & 21 \\
\hline 15 & 8 & 12.02.2010 & CL & S22 3300.5 W59 1709.1 & 116 & 80 & 70 & 50 & 60 & 8 & 3 & 1.5 & 24 \\
\hline 15 & 9 & 13.02.2010 & CL & S22 3300.6 W59 1708.5 & 103 & 75 & 30 & 60 & 50 & 8 & 3 & 1 & 22 \\
\hline 15 & 10 & 13.02 .2010 & CL & S22 3300.6 W59 1708.1 & 97 & 90 & 40 & 60 & 60 & 8 & 3 & 1 & 30 \\
\hline 15 & 17 & 18.02.2010 & $\mathrm{CM}$ & S22 3412.0 W59 2027.2 & 110 & 75 & 30 & 60 & 70 & 6 & 3 & 1.5 & 22 \\
\hline 15 & 179 & 10.12.2011 & YP & S21 3757.4 W58 5009.3 & 107 & 90 & 60 & 50 & 20 & 6 & 3.5 & 1.2 & 17 \\
\hline 15 & 180 & 10.12.2011 & YP & S21 3757.8 W58 5008.0 & 108 & 85 & 60 & 60 & 30 & 6 & 4 & 1.2 & 20 \\
\hline 15 & 181 & 10.12.2011 & YP & S21 3754.3 W58 5009.3 & 115 & 85 & 50 & 40 & 25 & 6 & 4 & 1.2 & 19 \\
\hline 15 & 182 & 10.12.2011 & YP & S21 3747.5 W58 5009.3 & 104 & 85 & 20 & 50 & 30 & 6 & 4 & 1.5 & 23 \\
\hline 15 & 204 & 10.01.2012 & FS & S23 0451.0 W59 1702.4 & 118 & 80 & 50 & 40 & 10 & 7 & 4 & 0.7 & 20 \\
\hline 15 & 207 & 10.01.2012 & FS & S23 0444.2 W59 1612.2 & 108 & 80 & 70 & 25 & 40 & 6.5 & 4 & 0.7 & 15 \\
\hline 16 & 25 & 19.03.2010 & CL & S22 3232.9 W59 1827.8 & 146 & 85 & & 75 & 30 & & 4 & 1.2 & 20 \\
\hline 16 & 28 & 19.02.2010 & $\mathrm{CM}$ & S22 3408.0 W59 2123.4 & 130 & 95 & & 75 & 60 & & 4 & 1.5 & 32 \\
\hline 16 & 63 & 08.12 .2010 & LG & S22 3520.1 W59 3507.6 & 120 & 80 & 60 & 10 & 30 & 6 & 3 & 1 & 24 \\
\hline 16 & 69 & 10.12 .2010 & LG & S22 3415.4 W59 3546.4 & 110 & 95 & 0 & 70 & 40 & & 4.5 & 1 & 20 \\
\hline 16 & 70 & 10.12 .2010 & LG & S22 3415.4 W59 3546.6 & 122 & 97 & 60 & 30 & 80 & 5 & 3 & 1.5 & 18 \\
\hline 16 & 71 & 10.12.2010 & LG & S22 3415.5 W59 3546.9 & 122 & 98 & 70 & 50 & 40 & 6 & 3.5 & 1.5 & 24 \\
\hline 16 & 72 & 10.12.2010 & LG & S22 3415.2 W59 3547.1 & 123 & 93 & 60 & 70 & 50 & 6 & 3 & 1.5 & 19 \\
\hline 16 & 125 & 19.01.2011 & $\mathrm{LC}$ & S22 3223.8 W59 4016.8 & 127 & 95 & 60 & 35 & 85 & 7 & 3.5 & 1.7 & 25 \\
\hline 16 & 130 & 19.01.2011 & $\mathrm{LC}$ & S22 3221.7 W59 4016.3 & 124 & 90 & 75 & 60 & 50 & 7 & 3.5 & 1.5 & 25 \\
\hline 16 & 131 & 19.01.2011 & $\mathrm{LC}$ & S22 3221.7 W59 4016.3 & 127 & 85 & 60 & 50 & 40 & 6 & 3.5 & 1.5 & 28 \\
\hline 16 & 132 & 19.01.2011 & LC & S22 3221.4 W59 4016.3 & 119 & 95 & 80 & 50 & 40 & 7 & 4 & 1.5 & 25 \\
\hline 16 & 133 & 19.01.2011 & $\mathrm{LC}$ & S22 3221.0 W59 4016.5 & 123 & 90 & 50 & 60 & 60 & 5.5 & 3.5 & 1.5 & 26 \\
\hline 16 & 142 & 23.11.2011 & $\mathrm{RF}$ & S20 4524.8 W59 2143.3 & 111 & 95 & 60 & 40 & 60 & 6.5 & 3.5 & 1 & 22 \\
\hline 16 & 145 & 23.11.2011 & $\mathrm{RF}$ & S20 4553.9 W59 2104.4 & 134 & 90 & 40 & 60 & 80 & 5 & 3.5 & 1 & 23 \\
\hline 16 & 146 & 23.11.2011 & RF & S20 4554.0 W59 2103.7 & 119 & 97 & 80 & 50 & 60 & 7 & 3.5 & 1 & 23 \\
\hline 16 & 147 & 24.11.2011 & $\mathrm{RF}$ & S20 4603.6 W59 2057.9 & 105 & 85 & 40 & 50 & 70 & 6.5 & 3.5 & 0.8 & 27 \\
\hline 16 & 187 & 08.11 .2011 & Cen & S22 0442.1 W59 0757.4 & 115 & 90 & 40 & 60 & 70 & 5 & 3.5 & 1 & 25 \\
\hline 16 & 188 & 08.11 .2011 & Cen & S22 0442.1 W59 0758.0 & 115 & 90 & 70 & 40 & 80 & 6.5 & 3.5 & 1 & 20 \\
\hline
\end{tabular}




\begin{tabular}{|c|c|c|c|c|c|c|c|c|c|c|c|c|c|}
\hline 16 & 191 & 06.01 .2012 & Cen & S21 5840.7 W59 0512.9 & 11 & 90 & & 70 & 85 & & 4 & 1 & 36 \\
\hline 16 & 196 & 17.01.2012 & Cen & S22 0223.7 W59 0524.2 & 109 & 90 & 20 & 70 & 70 & 6 & 4 & 1.5 & 19 \\
\hline 16 & 197 & 04.01.2012 & $\mathrm{RM}$ & S22 1452.4 W59 2755.8 & 118 & 95 & 25 & 85 & 30 & 5.5 & 3.5 & 0.6 & 30 \\
\hline 16 & 199 & 04.01 .2012 & $\mathrm{RM}$ & S22 1453.4 W59 2757.7 & 118 & 90 & 25 & 70 & 80 & 5.5 & 3.5 & 1.5 & 37 \\
\hline 16 & 216 & 24.10.2012 & $\mathrm{RM}$ & S22 1451.0 W59 2800.6 & 119 & 95 & 20 & 75 & 70 & 4 & 3.5 & 0.8 & 36 \\
\hline 16 & 217 & 24.10 .2012 & $\mathrm{RM}$ & S22 1450.7 W59 2800.2 & 127 & 85 & 30 & 50 & 75 & 6 & 3.5 & 1 & 32 \\
\hline 16 & 218 & 24.10 .2012 & $\mathrm{RM}$ & S22 1450.6 W59 2800.1 & 122 & 85 & 20 & 50 & 80 & 5 & 3.5 & 1.5 & 34 \\
\hline 16 & 219 & 24.10 .2012 & $\mathrm{RM}$ & S22 1451.4 W59 2759.2 & 118 & 95 & & 0 & 85 & 6 & 3.5 & 1.7 & 30 \\
\hline 16 & 220 & 24.10 .2012 & $\mathrm{RM}$ & S22 1451.9 W59 2759.0 & 111 & 93 & 50 & 70 & 75 & 6.5 & 3.5 & 1.7 & 27 \\
\hline 16 & 221 & 24.10 .2012 & $\mathrm{RM}$ & S22 1450.3 W59 2758.9 & 117 & 92 & 50 & 60 & 70 & 6.5 & 3.5 & 1.8 & 26 \\
\hline 16 & 222 & 24.10 .2012 & $\mathrm{RM}$ & S22 1449.5 W59 2758.9 & 126 & 85 & 50 & 50 & 80 & 6 & 3.5 & 1.5 & 30 \\
\hline 16 & 230 & 25.11.2012 & CL & S22 3234.7 W59 1832.1 & 116 & 85 & 5 & 80 & 40 & 5.5 & 3.5 & 1 & 21 \\
\hline 16 & 233 & 25.11.2012 & CL & S22 3218.4 W59 1846.3 & 123 & 85 & 6 & 75 & 30 & 4 & 3.5 & 1.4 & 19 \\
\hline 17 & 3 & 12.02.2010 & CL & S22 3259.8 W59 1710.9 & 115 & 80 & 70 & 50 & 50 & 7 & 3 & 1 & 22 \\
\hline 17 & 12 & 16.02.2010 & $\mathrm{CM}$ & S22 34 10.7 W59 2028.4 & 106 & 95 & 50 & 65 & 70 & 7 & 3 & 1 & 37 \\
\hline 17 & 13 & 16.02.2010 & $\mathrm{CM}$ & S22 3411.2 W59 2028.1 & 105 & 90 & 20 & 50 & 60 & 6.5 & 3 & 1.5 & 28 \\
\hline 17 & 14 & 16.02 .2010 & $\mathrm{CM}$ & S22 3411.4 W59 2028.1 & 126 & 85 & 50 & 40 & 50 & 7 & 3 & 1.5 & 25 \\
\hline 17 & 15 & 18.02.2010 & $\mathrm{CM}$ & S22 3411.7 W59 2027.8 & 115 & 95 & 80 & 70 & 70 & 6 & 3 & 1.5 & 25 \\
\hline 17 & 16 & 18.02.2010 & $\mathrm{CM}$ & S22 3411.7 W59 2027.3 & 98 & 90 & 60 & 30 & 70 & 6 & 3 & 1.5 & 21 \\
\hline 17 & 18 & 18.02.2010 & $\mathrm{CM}$ & S22 3412.3 W59 2027.1 & 104 & 85 & 20 & 50 & 70 & 6 & 3 & 1.5 & 26 \\
\hline 17 & 19 & 18.02.2010 & $\mathrm{CM}$ & S22 3412.9 W59 2027.1 & 115 & 70 & 40 & 50 & 50 & 7 & 3 & 1.5 & 20 \\
\hline 17 & 33 & 13.02.2010 & CL & S22 3254.2 W59 1841.2 & 105 & 60 & 40 & 30 & 40 & 5 & 3 & 1.2 & 24 \\
\hline 17 & 34 & 14.02 .2010 & CL & S22 3228.7 W59 1818.2 & 106 & 80 & 40 & 60 & 50 & 5 & 3 & 1.3 & 26 \\
\hline 17 & 35 & 14.02.2010 & CL & S22 3219.6 W59 1808.5 & 102 & 95 & 40 & 50 & 40 & 5 & 3 & 1 & 19 \\
\hline 17 & 36 & 15.02.2010 & CL & S22 3221.3 W59 1748.9 & 129 & 85 & 30 & 50 & 60 & 4.5 & 3 & 1.5 & 22 \\
\hline 17 & 37 & 19.03.2010 & CL & S22 3231.5 W59 1828.2 & 123 & 90 & 50 & 30 & 70 & 6 & 3 & 1.5 & 17 \\
\hline 17 & 38 & 19.03.2010 & CL & S22 3224.2 W59 1755.5 & 119 & 95 & 10 & 60 & 80 & 4.5 & 3 & 1.5 & 16 \\
\hline 17 & 39 & 19.02.2010 & $\mathrm{CM}$ & S22 3441.4 W59 2242.6 & 123 & 95 & 50 & 40 & 60 & 5 & 3 & 1.5 & 29 \\
\hline 17 & 40 & 22.03.2010 & $\mathrm{CM}$ & S22 3429.1 W59 2239.3 & 115 & 75 & 20 & 30 & 60 & 5 & 3 & 1.5 & 27 \\
\hline 17 & 41 & 14.02.2010 & CL & S22 3228.0 W59 1817.5 & 118 & 65 & 50 & 50 & 60 & 7 & 4 & 1.2 & 17 \\
\hline 17 & 42 & 13.02.2010 & CL & S22 3254.7 W59 1842.8 & 119 & 70 & 40 & 30 & 25 & 9 & 3 & 1.3 & 20 \\
\hline 17 & 43 & 14.02.2010 & CL & S22 3221.2 W59 1808.8 & 100 & 80 & 40 & 60 & 40 & 7 & 3 & 1.2 & 22 \\
\hline 17 & 44 & 19.03.2010 & CL & S22 3229.9 W59 1829.3 & 110 & 95 & 60 & 40 & 50 & 8 & 3 & 1.5 & 19 \\
\hline 17 & 45 & 19.03.2010 & CL & S22 3224.5 W59 1755.0 & 120 & 95 & 40 & 40 & 60 & 6 & 3 & 1.5 & 20 \\
\hline 17 & 46 & 19.02.2010 & $\mathrm{CM}$ & S22 3442.1 W59 2243.0 & 105 & 80 & 35 & 60 & 50 & 8 & 3 & 1.5 & 26 \\
\hline 17 & 47 & 19.02.2010 & $\mathrm{CM}$ & S22 3408.8 W59 2123.6 & 107 & 97 & 50 & 60 & 40 & 12 & 3 & 1.5 & 27 \\
\hline 17 & 48 & 20.02.2010 & $\mathrm{CM}$ & S22 3428.0 W59 2311.7 & 127 & 85 & 50 & 50 & 40 & 7 & 3 & 1.5 & 28 \\
\hline 17 & 49 & 20.02.2010 & $\mathrm{CM}$ & S22 3439.6 W59 2253.4 & 104 & 90 & 50 & 40 & 50 & 12 & 3 & 1.2 & 23 \\
\hline 17 & 50 & 20.02.2010 & $\mathrm{CM}$ & S22 3416.3 W59 2054.4 & 114 & 80 & 50 & 40 & 70 & 8 & 3.5 & 1.5 & 28 \\
\hline 17 & 67 & 08.12 .2010 & LG & S22 3503.3 W59 3512.2 & 135 & 85 & 50 & 20 & 40 & 8 & 3.5 & 1.5 & 25 \\
\hline 17 & 73 & 10.12.2010 & LG & S22 34 15.2 W59 3547.4 & 114 & 85 & 60 & 50 & 40 & 6 & 3.5 & 1.5 & 28 \\
\hline 17 & 74 & 10.12.2010 & LG & S22 3415.0 W59 3547.8 & 125 & 70 & 40 & 50 & 20 & 6.5 & 4 & 1.5 & 23 \\
\hline 17 & 75 & 10.12.2010 & LG & S22 3415.3 W59 3548.1 & 124 & 85 & 70 & 50 & 15 & 6 & 3.5 & 1.2 & 25 \\
\hline 17 & 76 & 10.12.2010 & LG & S22 34 15.2 W59 3548.4 & 127 & 85 & 15 & 70 & 15 & 6 & 4 & 1.2 & 21 \\
\hline 17 & 77 & 10.12.2010 & LG & S22 34 15.4 W59 3548.9 & 129 & 90 & 45 & 70 & 60 & 8 & 3.5 & 1.5 & 31 \\
\hline 17 & 78 & 10.12.2010 & LG & S22 3405.1 W59 3507.9 & 126 & 98 & 45 & 70 & 60 & 9 & 4 & 1.5 & 27 \\
\hline 17 & 80 & 14.12.2010 & LG & S22 3413.8 W59 3509.5 & 118 & 97 & 60 & 60 & 80 & 8 & 3.5 & 1.2 & 25 \\
\hline 17 & 86 & 15.12.2010 & LG & S22 3428.6 W59 3628.8 & 126 & 85 & 50 & 40 & 60 & 5 & 3.5 & 1.5 & 27 \\
\hline 17 & 87 & 15.12.2010 & LG & S22 3426.2 W59 3627.9 & 115 & 85 & 75 & 60 & 40 & 10 & 3.5 & 1 & 29 \\
\hline 17 & 93 & 15.12.2010 & LG & S22 3435.3 W59 3643.2 & 103 & 98 & 55 & 40 & 90 & 8 & 3.5 & 1 & 28 \\
\hline 17 & 98 & 05.01.2011 & RS & S22 3739.9 W59 0245.4 & 103 & 95 & 65 & 40 & 65 & 6 & 3.5 & 1.5 & 15 \\
\hline 17 & 99 & 05.01.2011 & RS & S22 3739.4 W59 0245.7 & 103 & 100 & 85 & 20 & 80 & 9 & 3 & 1.3 & 13 \\
\hline 17 & 100 & 06.01 .2011 & RS & S22 3740.8 W59 0345.0 & 108 & 80 & & 65 & 70 & & 4.5 & 1.2 & 31 \\
\hline 17 & 120 & 17.01.2011 & $\mathrm{LC}$ & S22 3221.5 W59 4019.8 & 126 & 97 & 50 & 40 & 90 & 6 & 3 & 1.5 & 25 \\
\hline 17 & 121 & 17.01.2011 & LC & S22 3220.9 W59 4019.4 & 117 & 97 & 80 & 40 & 80 & 7 & 4 & 1.5 & 28 \\
\hline 17 & 123 & 17.01.2011 & LC & S22 3241.8 W59 4008.8 & 122 & 95 & 70 & 50 & 85 & 6 & 3.5 & 1 & 18 \\
\hline 17 & 126 & 13.01.2011 & LC & S22 3223.1 W59 4016.5 & 122 & 80 & 40 & 60 & 85 & 7 & 4 & 1.8 & 26 \\
\hline 17 & 127 & 19.01.2011 & $\mathrm{LC}$ & S22 3222.9 W59 4016.5 & 122 & 98 & 90 & 20 & 95 & 8 & 3.5 & 1.7 & 31 \\
\hline 17 & 128 & 19.01.2011 & $\mathrm{LC}$ & S22 3222.6 W59 4016.2 & 119 & 90 & 40 & 50 & 70 & 6 & 3.5 & 1 & 25 \\
\hline
\end{tabular}




\begin{tabular}{|c|c|c|c|c|c|c|c|c|c|c|c|c|c|}
\hline 17 & 129 & 19.01 .2011 & $\mathrm{LC}$ & S22 3222.3 W59 4015.9 & 123 & 95 & 60 & 40 & 60 & 6 & 3 & 1.5 & 24 \\
\hline 17 & 138 & 26.01.2011 & LE & S22 3332.0 W59 2823.4 & 112 & 97 & 60 & 70 & 85 & 9 & 3.5 & 1.5 & 26 \\
\hline 17 & 141 & 23.11.2011 & RF & S20 45 23.9 W59 2143.8 & 108 & 95 & 25 & 80 & 70 & 5 & 3.5 & 1 & 25 \\
\hline 17 & 148 & 24.11.2011 & RF & S20 4603.4 W59 2057.7 & 110 & 90 & 60 & 30 & 80 & 6 & 3.5 & 1.5 & 21 \\
\hline 17 & 149 & 24.11.2011 & RF & S20 4603.3 W59 2057.5 & 109 & 90 & 70 & 30 & 70 & 6.5 & 2.5 & 1 & 23 \\
\hline 17 & 150 & 24.11.2011 & $\mathrm{RF}$ & S20 4603.0 W59 2057.5 & 115 & 92 & 70 & 40 & 70 & 7 & 3.5 & 1.2 & 23 \\
\hline 17 & 151 & 24.11.2011 & RF & S20 4602.7 W59 2057.6 & 106 & 95 & 80 & 40 & 75 & 7 & 3.5 & 1 & 23 \\
\hline 17 & 152 & 24.11.2011 & RF & S20 4602.1 W59 2057.6 & 108 & 85 & 75 & 30 & 50 & 6 & 3.5 & 0.8 & 22 \\
\hline 17 & 153 & 24.11.2011 & $\mathrm{RF}$ & S20 4602.0 W59 2057.4 & 121 & 85 & 80 & 15 & 25 & 5.5 & 3.5 & 0.6 & 15 \\
\hline 17 & 154 & 24.11.2011 & RF & S20 4601.7 W59 2057.2 & 105 & 90 & 80 & 30 & 40 & 6.5 & 3.5 & 0.6 & 21 \\
\hline 17 & 155 & 24.11.2011 & RF & S20 4601.3 W59 2057.1 & 118 & 93 & 80 & 50 & 85 & 7 & 3.5 & 1 & 26 \\
\hline 17 & 156 & 24.11.2011 & RF & S20 4601.1 W59 2057.0 & 119 & 95 & 75 & 40 & 85 & 6 & 3.5 & 0.8 & 25 \\
\hline 17 & 160 & 25.11.2011 & RF & S20 45 14.6 W59 2210.4 & 119 & 90 & 85 & 40 & 75 & 7 & 3.5 & 1.5 & 23 \\
\hline 17 & 202 & 04.01 .2012 & $\mathrm{RM}$ & S22 1458.5 W59 2755.9 & 124 & 97 & 85 & 40 & 90 & 9 & 4 & 1.2 & 29 \\
\hline 17 & 210 & 11.01.2012 & FS & S23 0434.6 W59 1243.3 & 109 & 90 & 50 & 60 & 70 & 6 & 4 & 1.5 & 28 \\
\hline 17 & 228 & 15.11.2012 & $\mathrm{CM}$ & S22 3419.5 W59 2022.2 & 116 & 98 & 80 & 50 & 90 & 8 & 4 & 1.2 & 18 \\
\hline 17 & 234 & 25.11 .2012 & CL & S22 3148.0 W59 1847.1 & 115 & 97 & 75 & 60 & 80 & 8 & 3.5 & 1.6 & 27 \\
\hline 18 & 51 & 13.02.2010 & CL & S22 3254.9 W59 1843.5 & 116 & 85 & 40 & 60 & 40 & 10 & 3 & 1 & 22 \\
\hline 18 & 52 & 19.02.2010 & $\mathrm{CM}$ & S22 34 09.4 W59 2122.7 & 108 & 95 & 40 & 70 & 50 & 10 & 3 & 1.5 & 26 \\
\hline 18 & 53 & 20.02.2010 & $\mathrm{CM}$ & S22 3429.7 W59 2312.5 & 117 & 80 & 40 & 30 & 70 & 8 & 3 & 1.5 & 32 \\
\hline 18 & 54 & 20.02.2010 & $\mathrm{CM}$ & S22 3418.5 W59 2053.4 & 121 & 95 & 75 & 50 & 50 & 10 & 3 & 1.5 & 27 \\
\hline 18 & 55 & 22.03.2010 & $\mathrm{CM}$ & S22 3445.5 W59 2245.4 & 115 & 90 & 60 & 50 & 60 & 8 & 3 & 1.5 & 21 \\
\hline 18 & 82 & 14.12.2010 & LG & S22 3416.7 W59 3512.5 & 118 & 85 & 70 & 60 & 70 & 8 & 4 & 1.5 & 27 \\
\hline 18 & 89 & 15.12 .2010 & LG & S22 3421.3 W59 3641.4 & 120 & 98 & 85 & 30 & 40 & 10 & 4 & 1.5 & 28 \\
\hline 18 & 101 & 06.01 .2011 & RS & S22 3741.0 W59 0346.5 & 117 & 100 & 80 & 50 & 80 & 7 & 4 & 1.5 & 40 \\
\hline 18 & 103 & 06.01 .2011 & RS & S22 3739.0 W59 0304.3 & 106 & 100 & 75 & 40 & 85 & 8.5 & 3.5 & 1.5 & 25 \\
\hline 18 & 107 & 07.01.2011 & RS & S22 3747.3 W59 0237.6 & 107 & 95 & 80 & 40 & 60 & 8 & 3.5 & 1.5 & 35 \\
\hline 18 & 108 & 07.01.2011 & RS & S22 3747.3 W59 0237.5 & 100 & 95 & 75 & 30 & 90 & 8 & 3 & 1.8 & 38 \\
\hline 18 & 109 & 07.01.2011 & RS & S22 3747.3 W59 0237.0 & 93 & 97 & 40 & 50 & 85 & 7 & 4 & 1.5 & 35 \\
\hline 18 & 110 & 07.01.2011 & RS & S22 3747.8 W59 0236.4 & 116 & 95 & 60 & 50 & 70 & 7 & 4 & 1.9 & 36 \\
\hline 18 & 111 & 07.01.2011 & RS & S22 3748.0 W59 0236.4 & 106 & 95 & 40 & 60 & 90 & 6.5 & 4 & 1.8 & 31 \\
\hline 18 & 112 & 07.01.2011 & RS & S22 3748.3 W59 0236.1 & 107 & 85 & 40 & 40 & 65 & 6 & 4 & 1.5 & 28 \\
\hline 18 & 113 & 07.01.2011 & RS & S22 3748.8 W59 0235.4 & 107 & 85 & 40 & 40 & 60 & 6 & 4 & 1.5 & 34 \\
\hline 18 & 173 & 08.12.2011 & YP & S21 4130.8 W58 4053.1 & 105 & 97 & 60 & 50 & 30 & 9 & 4 & 1.3 & 28 \\
\hline 18 & 174 & 09.12.2011 & YP & S21 35 47.6 W58 5344.6 & 114 & 90 & 40 & 60 & 60 & 7 & 4 & 1.5 & 17 \\
\hline 18 & 175 & 09.12.2011 & YP & S21 3434.6 W58 5345.9 & 105 & 95 & 50 & 50 & 80 & 6 & 4 & 1.5 & 15 \\
\hline 18 & 176 & 09.12.2011 & YP & S21 3436.4 W58 5347.4 & 103 & 95 & 70 & 40 & 60 & 11 & 4 & 1.5 & 23 \\
\hline 18 & 177 & 09.12.2011 & YP & S21 4126.5 W58 5057.3 & 109 & 95 & 80 & 40 & 50 & 8 & 4 & 1 & 34 \\
\hline 18 & 178 & 09.12.2011 & YP & S21 4125.6 W58 5057.2 & 101 & 95 & 70 & 30 & 75 & 7 & 4 & 1 & 24 \\
\hline 18 & 183 & 10.12.2011 & YP & S21 38 09.4 W58 5213.5 & 99 & 75 & 50 & 40 & 60 & 6 & 4 & 1.5 & 32 \\
\hline 18 & 184 & 10.12.2011 & YP & S21 3809.0 W58 5213.1 & 94 & 80 & 80 & 40 & 25 & 4 & 4 & 1.5 & 23 \\
\hline 18 & 185 & 10.12.2011 & YP & S21 3808.1 W58 5213.7 & 99 & 90 & 40 & 60 & 70 & 6.5 & 4 & 1.5 & 34 \\
\hline 18 & 192 & 17.01.2012 & Cen & S21 5836.5 W59 0508.9 & 105 & 95 & 70 & 40 & 85 & 8 & 4 & 1.7 & 38 \\
\hline 18 & 193 & 17.01.2012 & Cen & S21 5837.0 W59 0508.7 & 121 & 90 & 80 & 40 & 60 & 9 & 4 & 1.5 & 39 \\
\hline 18 & 194 & 17.01.2012 & Cen & S21 5838.3 W59 0510.0 & 118 & 98 & 85 & 60 & 98 & 10 & 4.5 & 1.8 & 35 \\
\hline 18 & 195 & 17.01.2012 & Cen & S22 0224.6 W59 0521.1 & 111 & 98 & 85 & 70 & 90 & 10 & 4 & 1.5 & 36 \\
\hline 18 & 198 & 04.01 .2012 & $\mathrm{RM}$ & S22 1451.8 W59 2755.4 & 120 & 96 & 60 & 60 & 80 & 6.5 & 4 & 1.6 & 37 \\
\hline 18 & 200 & 04.01 .2012 & $\mathrm{RM}$ & S22 1453.6 W59 2758.0 & 126 & 95 & 70 & 40 & 85 & 6 & 4 & 1.5 & 32 \\
\hline 18 & 212 & 11.01.2012 & FS & S23 04 17.2 W59 1240.1 & 126 & 95 & 70 & 70 & 80 & 8 & 4 & 1.5 & 36 \\
\hline 18 & 215 & 13.01.2012 & $\mathrm{RV}$ & S23 1258.0 W59 1200.2 & 112 & 90 & 60 & 60 & 80 & 7 & 4 & 1.5 & 46 \\
\hline 18 & 223 & 24.10 .2012 & $\mathrm{RM}$ & S22 1449.6 W59 2758.7 & 118 & 95 & 70 & 60 & 80 & 9 & 3.5 & 1.2 & 28 \\
\hline 18 & 240 & 22.11.2012 & RG & S22 4242.8 W59 2745.2 & 116 & 95 & 80 & 40 & 40 & 7 & 3.5 & 1.2 & 30 \\
\hline 19 & 56 & 22.03.2010 & $\mathrm{CM}$ & S22 3428.7 W59 2053.1 & 111 & 90 & 30 & & 90 & 7 & & 1.5 & 34 \\
\hline 19 & 57 & 22.03.2010 & $\mathrm{CM}$ & S22 3425.9 W59 2053.7 & 123 & 97 & 80 & 30 & 85 & 9 & 2.5 & 1.5 & 30 \\
\hline 19 & 58 & 22.03.2010 & $\mathrm{CM}$ & S22 3425.9 W59 2030.2 & 114 & 70 & 20 & & 50 & 6.5 & & 1.5 & 18 \\
\hline 19 & 59 & 22.03.2010 & $\mathrm{CM}$ & S22 3430.5 W59 2028.9 & 116 & 70 & & & 70 & & & 1.5 & 11 \\
\hline 19 & 60 & 22.03.2010 & $\mathrm{CM}$ & S22 3430.1 W59 2052.1 & 117 & 80 & 10 & 6 & 70 & 7 & 3 & 1.5 & 26 \\
\hline 19 & 61 & 22.03.2010 & $\mathrm{CM}$ & S22 3429.5 W59 2051.8 & 120 & 80 & 25 & 10 & 80 & 7 & 2 & 1.2 & 23 \\
\hline 19 & 64 & 08.12 .2010 & LG & S22 3450.4 W59 3553.3 & 120 & 65 & 40 & 5 & 30 & 9 & 2 & 0.8 & 18 \\
\hline
\end{tabular}




\begin{tabular}{|c|c|c|c|c|c|c|c|c|c|c|c|c|c|}
\hline \multirow{2}{*}{$\begin{array}{l}\text { Ein } \\
\text { heit }\end{array}$} & \multirow{2}{*}{$\begin{array}{l}\text { Prob. } \\
\text { fl. }\end{array}$} & \multirow[t]{2}{*}{ Datum } & \multirow[t]{2}{*}{ Ort } & \multirow[t]{2}{*}{ Koordinaten } & \multirow{2}{*}{$\begin{array}{l}\text { Höhe } \\
\text { NN }\end{array}$} & \multicolumn{4}{|c|}{ Deckung - Vegetation } & \multicolumn{3}{|c|}{ Höhe - Vegetation } & \multirow{2}{*}{$\begin{array}{l}\text { Art- } \\
\mathrm{Nr}\end{array}$} \\
\hline & & & & & & Total & Baum & Strauch & Kraut & Baum & Strauch & Kraut & \\
\hline 19 & 81 & 14.12 .2010 & LG & S22 3414.6 W59 3510.5 & 119 & 70 & 45 & 2 & 50 & 6 & 3.5 & 1 & 15 \\
\hline 19 & 83 & 14.12 .2010 & LG & S22 3427.2 W59 3520.9 & 121 & 85 & 50 & 15 & 70 & 12 & 4 & 0.8 & 28 \\
\hline 19 & 90 & 15.12 .2010 & LG & S22 3421.9 W59 3645.7 & 107 & 50 & 40 & 30 & 30 & 8 & 3 & 1 & 25 \\
\hline 19 & 114 & 08.01.2011 & RS & S22 37 19.4 W59 0630.9 & 112 & 80 & 25 & 10 & 70 & 7 & 2.3 & 1.4 & 35 \\
\hline 19 & 115 & 08.01 .2011 & RS & S22 3721.1 W59 0634.7 & 113 & 90 & 80 & 15 & 85 & 10 & 2.5 & 1.4 & 26 \\
\hline 19 & 134 & 26.01 .2011 & LC & S22 3226.9 W59 4013.1 & 125 & 50 & 5 & 20 & 30 & 5 & 3 & 1 & 13 \\
\hline 19 & 135 & 26.01.2011 & LC & S22 3229.6 W59 4010.1 & 118 & 70 & 35 & 20 & 60 & 6.5 & 3 & 1.5 & 10 \\
\hline 19 & 136 & 26.01.2011 & LC & S22 3235.6 W59 4021.6 & 116 & 80 & 25 & 30 & 70 & 6 & 2.5 & 1.2 & 14 \\
\hline 19 & 224 & 15.11 .2012 & $\mathrm{CM}$ & S22 3428.2 W59 2051.9 & 127 & 95 & & 7 & 85 & & 2.5 & 1.2 & 28 \\
\hline
\end{tabular}


Anhang 5-2: Synoptische Tabelle aller vorkommenden Arten in den Gehölzgesellschaften. Genannt werden die Abkürzungen, Trivialnamen und Frequenzklassen der Arten in den jeweiligen Gesellschaften.

\begin{tabular}{|c|c|c|c|c|c|c|c|c|c|c|c|c|}
\hline \multirow{2}{*}{ Arten } & \multirow{2}{*}{ Abkürzung } & \multirow{2}{*}{ Trivialname } & \multicolumn{10}{|c|}{ Gehölzgesellschaften } \\
\hline & & & 10 & 11 & 12 & 13 & 14 & 15 & 16 & 17 & 18 & 19 \\
\hline \multicolumn{13}{|l|}{ BAUMSCHICHT } \\
\hline Acacia praecox & ACAPRAE1 & Jukeri hû & & & & & & & & I & II & \\
\hline Acanthosyris falcata & ACAFALC1 & Saucillo, yva he'e & & & & & & & & & I & \\
\hline Anisocapparis speciosa & ANISPEC1 & Pajagua naranja & & & & & & & & & I & \\
\hline Aspidosperma quebracho-blanco & ASPQUBL1 & Quebracho blanco & & & & & & & I & I & III & \\
\hline Aspidosperma triternatum & ASPTRIT1 & Quebrachillo blanco & & & & & & & & & I & \\
\hline Astronium fraxinifolium & ASTFRAX1 & Urunde’y para & & & & & & & & & & II \\
\hline Bougainvillea campanulata & BOUCAMP1 & Gallo espuela & & & & & & & II & I & I & \\
\hline Bulnesia bonariensis & BULBONA1 & & & & & & & & & I & & \\
\hline Bulnesia sarmientoi & BULSARM1 & Palo santo & & & & I & & & I & IV & II & \\
\hline Caesalpinia paraguariensis & CAEPARA1 & Guayacán & & & & & & II & & I & I & \\
\hline Ceiba chodatii & CEICHOD1 & Samu’u, palo borracho & & & & & & $\mathrm{I}$ & I & I & II & \\
\hline Cercidium praecox & CERPRAE1 & Verde olivo & & & & & & & & I & & \\
\hline Cereus forbesii & CERFORB1 & Tuna & & & & & & & I & & & \\
\hline Cereus stenogonus & CERSTEN1 & Tuna, candelabro & & & & & & & & & I & \\
\hline Chloroleucon tenuiflorum & CHLTENU1 & Tataré & & & & & & & & I & & \\
\hline Coccoloba cordata & COCCORD1 & Duraznillo morado & & & & & & & & & I & \\
\hline Copernicia alba & COPALBA1 & Karanda’y & II & II & & & & & & & & \\
\hline Cordia americana & CORAMER1 & Guajayvi & & & & & & & & & I & \\
\hline Cordia glabrata & CORGLAB1 & Peterevy moroti & & & & & & & & & I & \\
\hline Cynophalla retusa & CYNRETU1 & Indio kumanda & & & & & & & & I & II & \\
\hline Diplokeleba floribunda & DIPFLOR1 & Palo piedra & & & & & & & & & I & \\
\hline Geoffroea spinosa & GEOSPIN1 & Guaikuru manduvi & & & & & & & & & I & \\
\hline Lycium boerhaaviaefolium & LYCBOER1 & Tala salada & & & & & & & I & & & \\
\hline Microlobius foetidus s. paraguensis & MICFOET1 & Yvyra ne & & & & & & & & & I & \\
\hline Mimozyganthus carinatus & MIMCARI1 & Jukeri pyta & & & & & I & & & I & & \\
\hline Parkinsonia aculeata & PARACUL1 & Cina cina & I & & & & & & & & & \\
\hline Phyllostylon rhamnoides & PHYRHAM1 & Palo lanza & & & & & & & & I & III & \\
\hline Piptadeniopsis lomentifera & PIPLOME1 & & & & & & & & II & I & I & \\
\hline Pisonia zapallo & PISZAPA1 & Jukyry vusu & & & & & & & & & I & \\
\hline Prosopis hassleri & PROHASS1 & & & & & & & & I & & & \\
\hline Prosopis kuntzei & PROKUNT1 & Karanda & & & & & & & & I & I & \\
\hline Prosopis rojasiana & PROROJA1 & & & & & & & & I & I & & \\
\hline Prosopis ruscifolia & PRORUSC1 & Viñal & & II & III & II & I & $\mathrm{V}$ & I & I & I & \\
\hline Prosopis sericantha & PROSERI1 & & & & & & & & I & I & & \\
\hline Quiabentia verticillata & QUIVERT1 & & & & & & & & I & & & \\
\hline Salta triflora & SALTRIF1 & Guaimi pire & & & & & & IV & III & III & $\mathrm{V}$ & \\
\hline Sarcotoxicum salicifolium & SARSALI1 & Sacha sandía & & & & & & & & I & & \\
\hline Schinopsis cornuta & SCHCORN1 & Urunde'y boli & & & & & & & & & & IV \\
\hline Schinopsis lorentzii & SCHLORE1 & Coronillo & & & & & & & & & I & \\
\hline Sideroxylon obtusifolium & SIDOBTU1 & Guajayvi ra’i & & & & & & & I & II & II & \\
\hline Stetsonia coryne & STECORY1 & Cardón, tuna & & & & & & & I & I & II & \\
\hline Tabebuia aurea & TABAURE1 & Paratodo & & & & & & & & & & II \\
\hline Tabebuia nodosa & TABNODO1 & Labón, palo cruz & & & I & I & & $\mathrm{V}$ & I & II & II & \\
\hline Trithrinax schyzophylla & TRISCHY1 & Karandilla & & & & & & & & I & III & \\
\hline Ximenia americana & XIMAMER1 & $\begin{array}{l}\text { Pata de monte, indio } \\
\text { kurupa'y }\end{array}$ & & & & & & & I & I & I & \\
\hline Ziziphus mistol & ZIZMIST1 & Mistol & & & & & I & & III & III & III & \\
\hline STRAUCHSCHICHT & & & & & & & & & & & & \\
\hline Acacia aroma & ACAAROM4 & Aromita & & & & & & & & & & III \\
\hline Acacia caven & ACACAVE4 & Aromita & & I & II & I & & & & & & \\
\hline Acacia curvifructa & ACACURV4 & & & & & & & & & & I & \\
\hline Acacia praecox & ACAPRAE4 & Jukeri hû & & & I & I & II & II & IV & IV & $\mathrm{V}$ & \\
\hline
\end{tabular}




\begin{tabular}{|c|c|c|c|c|c|c|c|c|c|c|c|c|}
\hline \multirow{2}{*}{ Arten } & \multirow{2}{*}{ Abkürzung } & \multirow{2}{*}{ Trivialname } & \multicolumn{10}{|c|}{ Gehölzgesellschaften } \\
\hline & & & 10 & 11 & 12 & 13 & 14 & 15 & 16 & 17 & 18 & 19 \\
\hline Acanthosyris falcata & ACAFALC4 & Saucillo, yva he'e & & & & & & & I & & & \\
\hline Achatocarpus praecox & ACHPRAE4 & Yvyra hû, arajú & & & & & I & III & I & I & III & \\
\hline Aloysia virgata & ALOVIRG4 & & & & & & & & & & I & I \\
\hline Amphilophium cynanchoides & AMPCYNA4 & & & & & & & & & I & I & \\
\hline Anisocapparis speciosa & ANISPEC4 & Pajagua naranja & & & & & & & II & I & III & \\
\hline Aspidosperma quebracho-blanco & ASPQUBL4 & Quebracho blanco & & & & & & I & & I & $\mathrm{I}$ & \\
\hline Aspidosperma triternatum & ASPTRIT4 & Quebrachillo blanco & & & & & & & & & I & \\
\hline Astronium fraxinifolium & ASTFRAX4 & Urunde'y para & & & & & & & & & & I \\
\hline Atriplex eximia & ATREXIM4 & & & & & I & & & & & & \\
\hline Basistemon spinosus & BASSPINO4 & & & & I & & & I & & & & I \\
\hline Bauhinia argentinensis & BAUARGE4 & & & & & & & & & & & $\mathrm{I}$ \\
\hline Bougainvillea campanulata & BOUCAMP4 & Gallo espuela & & & I & & I & III & III & III & II & \\
\hline Bulnesia bonariensis & BULBONA4 & & & & & & I & & I & & & \\
\hline Bulnesia sarmientoi & BULSARM4 & Palo santo & & & I & I & I & & I & II & & \\
\hline Caesalpinia paraguariensis & CAEPARA4 & Guayacán & & & & I & & I & & I & & \\
\hline Capparicordis tweediana & CAPTWEE4 & Sacha membrillo & & & III & I & II & II & III & II & IV & \\
\hline Cardiospermum halicacabum & CARHALI4 & Kamuvu & & & & & & & & & I & \\
\hline Castela coccinea & CASCOCC4 & Mistol del zorro & & & I & & & I & I & I & I & I \\
\hline Ceiba chodatii & CEICHOD4 & Samu’u & & & & & & & I & I & I & \\
\hline Celtis ehrenbergiana & CELEHRE4 & Juasy’y, tala & & & I & & I & I & II & III & III & I \\
\hline Cercidium praecox & CERPRAE4 & Verde olivo & & & I & & & & & & I & \\
\hline Cereus forbesii & CERFORB4 & Tuna & & & & & & & II & I & I & II \\
\hline Chloroleucon tenuiflorum & CHLTENU4 & Tataré & & & & & & & & I & & \\
\hline Cissus verticillata & CISVERT4 & & & & & & & & & I & I & \\
\hline Coccoloba cordata & COCCORD4 & Duraznillo morado & & & I & & & & & & I & \\
\hline Coccoloba spinescens & COCSPIN4 & & & & I & & & I & & & I & \\
\hline Copernicia alba & COPALBA4 & Karanda’y & II & I & I & & & & & I & & \\
\hline Cordia americana & CORAMER4 & Guajayvi & & & & & & & & & I & \\
\hline Cyclolepis genistoides & CYCGENI4 & Palo azul & & & & I & IV & & I & & & \\
\hline Cynophalla retusa & CYNRETU4 & Indio kumanda & & I & III & I & III & $\mathrm{V}$ & $\mathrm{V}$ & IV & $\mathrm{V}$ & I \\
\hline Diplokeleba floribunda & DIPFLOR4 & Palo piedra & & & & & & & & I & I & I \\
\hline Ditaxis montevidensis & DITMONT4 & & & & & & & & & & I & \\
\hline Erythroxylum cuneifolium & ERYCUNE4 & Coca del campo & & & & & & & & & I & \\
\hline Fridericia dichotoma & FRIDICH4 & & & & & & & & I & I & I & I \\
\hline Fridericia truncata & FRITRUN4 & & & & & & & I & I & I & I & \\
\hline Funastrum clausum & FUNCLAU4 & & III & I & & & & & & & & \\
\hline Guettarda uruguensis & GUEURUG4 & Jarmín del monte & & & & & & & & & I & \\
\hline Harrisia bonplandii & HARBOND4 & & & I & I & I & I & III & II & I & I & \\
\hline Herreria montevidensis & HERMONT4 & & & & & & & & & & II & \\
\hline Holmbergia tweedii & HOLTWEE4 & & & & & & I & & I & & & \\
\hline Ipomoea amnicola & IPOAMNI4 & & & & & & I & & I & I & I & \\
\hline Jatropha hieronymi & JATHIER4 & & & & & & & & & I & & \\
\hline Justicia xylosteoides & JUSXYLO4 & & & & & & I & & I & & & \\
\hline Lippia alba & LIPALBA4 & & & & & & & & & & I & \\
\hline Lophocarpinia aculeatifolia & LOPACUL4 & Hueso de sapo & & & & II & III & & I & I & & \\
\hline Lycium americanum & LYCAMER4 & & & & I & $\mathrm{V}$ & $\mathrm{V}$ & I & III & I & & \\
\hline Lycium boerhaaviaefolium & LYCBOER4 & Tala salada & & I & I & II & III & I & II & I & I & \\
\hline Lycium cuneatum & LYCCUNE4 & & & I & & & & & & I & I & \\
\hline Lycium morongii & LYCMORO4 & & & & I & & & & & & & \\
\hline Lycium nodosum & LYCNODO4 & & & II & I & & & & & & & \\
\hline Marsdenia castillonii & MARCAST4 & & & & & & I & & II & I & I & \\
\hline Maytenus vitis-idaea & MAYVITI4 & Sal de indio & & I & II & $\mathrm{V}$ & $\mathrm{V}$ & IV & $\mathrm{V}$ & $\mathrm{V}$ & II & \\
\hline Mimosa detinens & MIMDETI4 & Jukeri & & & I & I & I & & I & I & I & \\
\hline Mimozyganthus carinatus & MIMCARI4 & Jukeri pyta & & & & I & $\mathrm{I}$ & & I & I & & \\
\hline Monvillea spegazzinii & MONSPEG4 & & & & & & & & & I & & \\
\hline Opuntia anacantha $v$. retrorsa & OPURETR4 & & & & & & & & I & & & \\
\hline
\end{tabular}




\begin{tabular}{|c|c|c|c|c|c|c|c|c|c|c|c|c|}
\hline \multirow{2}{*}{ Arten } & \multirow{2}{*}{ Abkürzung } & \multirow{2}{*}{ Trivialname } & \multicolumn{10}{|c|}{ Gehölzgesellschaften } \\
\hline & & & 10 & 11 & 12 & 13 & 14 & 15 & 16 & 17 & 18 & 19 \\
\hline Parkinsonia aculeata & PARACUL4 & Cina cina & $\mathrm{V}$ & $\mathrm{I}$ & & & & & & & & \\
\hline Passiflora mooreana & PASMOOR4 & & & I & & & & & & & & \\
\hline Phyllostylon rhamnoides & PHYRHAM4 & Palo lanza & & & & & & & & & II & \\
\hline Piptadeniopsis lomentifera & PIPLOME4 & & & & & II & II & & III & II & I & I \\
\hline Pisonia hassleriana & PISHASS4 & & & & & & & & & & I & \\
\hline Pisonia zapallo & PISZAPA4 & Jukyry vusu & & & & & & & & & I & \\
\hline Prosopis hassleri & PROHASSL4 & & & & $\mathrm{I}$ & $\mathrm{I}$ & & & & & & I \\
\hline Prosopis kuntzei & PROKUNT4 & Karanda & & & & & & & & I & I & \\
\hline Prosopis nigra & PRONIGR4 & Algarrobo negro & & & I & & & & & & & \\
\hline Prosopis rojasiana & PROROJA4 & & & & & II & I & & II & I & & \\
\hline Prosopis ruscifolia & PRORUSC4 & Viñal & II & $\mathrm{V}$ & $\mathrm{V}$ & $\mathrm{V}$ & IV & & II & I & & \\
\hline Prosopis sericantha & PROSERI4 & & & & $\mathrm{I}$ & & I & I & I & I & & \\
\hline Pterogyne nitens & PTENITE4 & Yvyrarõ & & & & & & & & & & I \\
\hline Quiabentia verticillata & QUIVERT4 & & & & & & I & & & & & I \\
\hline Salta triflora & SALTRIF4 & Guaimi pire & & & I & & I & $\mathrm{V}$ & III & IV & III & \\
\hline Sarcotoxicum salicifolium & SARSALI4 & Sacha sandía & & & & & I & & II & II & II & \\
\hline Schaefferia argentinensis & SCHARGE4 & & & & & & & & & & I & \\
\hline Schinopsis cornuta & SCHCORN4 & Urunde'y boli & & & & & & & & & & II \\
\hline Schubertia grandiflora & SCHGRAN4 & & & & & & & & & & I & \\
\hline Sideroxylon obtusifolium & SIDOBTU4 & Guajayvi ra’i & & I & & & I & & II & II & II & \\
\hline Solanum glaucophyllum & SOLGLAU4 & & & I & & & & & & & & \\
\hline Stetsonia coryne & STECORY4 & Cardón, tuna & & & & & I & I & I & I & I & \\
\hline Tabebuia aurea & TABAURE4 & Paratodo & & & & & & & & & & I \\
\hline Tabebuia nodosa & TABNODO4 & Labón, palo cruz & & I & II & I & I & II & I & I & I & \\
\hline Trithrinax schyzophylla & TRISCHY4 & karandilla & & & & I & I & I & III & III & IV & \\
\hline Ximenia americana & XIMAMER4 & $\begin{array}{l}\text { Pata de monte, indio } \\
\text { kurupa'y }\end{array}$ & & & & & & & I & I & I & I \\
\hline Ziziphus mistol & ZIZMIST4 & Mistol & & & II & II & II & & IV & III & II & I \\
\hline KRAUTSCHICHT & & & & & & & & & & & & \\
\hline Abildgaardia ovata & ABIOVAT6 & & & & & & & & & & & I \\
\hline Acacia aroma & ACAAROM6 & Aromita & & & & & & & & & & III \\
\hline Acacia caven & ACACAVE6 & Aromita & & I & II & I & & & & & & \\
\hline Acacia praecox & ACAPRAE6 & Jukeri hû & & & & $\mathrm{I}$ & I & II & III & IV & IV & \\
\hline Achatocarpus praecox & ACHPRAE6 & Yvyra hú & & & & & I & & & I & I & \\
\hline $\begin{array}{l}\text { Aechmea distichantha } v \text {. } \\
\text { schlumbergeri }\end{array}$ & AECDIST6 & & & & & & & II & I & I & II & \\
\hline Aeschynomene histrix & AESHIST6 & & & & & & & & & & & I \\
\hline Aeschynomene mollicula & AESMOLL6 & & & & & & & & & & I & \\
\hline Aeschynomene viscidula & AESVISC6 & & & & & & & & & & & II \\
\hline Aloysia gratissima & ALOGRAT6 & & & & & & & & & I & & \\
\hline Aloysia virgata & ALOVIRG6 & Niño rupá, poleo & & & & & & & I & & & I \\
\hline Alternanthera kurtzii & ALTKURT6 & & & I & & I & & I & II & II & I & \\
\hline Alternanthera nodifera & ALTNODI6 & & & & & I & $\mathrm{I}$ & & & & & \\
\hline $\begin{array}{l}\text { Alternanthera paronychioides s. } \\
\text { chacoensis }\end{array}$ & ALTPARO6 & & II & II & & I & & & I & & & \\
\hline Amaranthus hybridus & AMAHYBR6 & & $\mathrm{I}$ & & & & & & & & & \\
\hline Amaranthus standleyanus & AMASTAN6 & & & & & & $\mathrm{I}$ & & I & & & \\
\hline Amaranthus viridis & AMAVIRI6 & & I & & & & & & & I & & \\
\hline Amphilophium cynanchoi & AMPCYNA6 & & & & & & & & & & I & \\
\hline Anemia tomentosa v. anthriscifolia & ANETOME6 & & & & & & & & & & I & \\
\hline Anisocapparis speciosa & ANISPEC6 & Pajagua naranja & & & $\mathrm{I}$ & & & & I & I & II & \\
\hline Araujia herzogii & ARAHERZ6 & & & & I & & & & & & & \\
\hline Araujia odorata & ARAODOR6 & & & & & & $\mathrm{I}$ & & & I & & I \\
\hline Araujia variegata & ARAVARI6 & & & & & & & & I & I & & \\
\hline Aristida mendocina & ARIMEND6 & & & & & & & & & & & IV \\
\hline
\end{tabular}




\begin{tabular}{|c|c|c|c|c|c|c|c|c|c|c|c|c|}
\hline \multirow{2}{*}{ Arten } & \multirow{2}{*}{ Abkürzung } & \multirow{2}{*}{ Trivialname } & \multicolumn{10}{|c|}{ Gehölzgesellschaften } \\
\hline & & & 10 & 11 & 12 & 13 & 14 & 15 & 16 & 17 & 18 & 19 \\
\hline Aspidosperma quebracho-blanco & ASPQUBL6 & Quebracho blanco & & & II & & & I & I & I & II & \\
\hline Aspilia pascalioides & ASPPASC6 & & & & & & & & & & & I \\
\hline Astraea lobata & ASTLOBA6 & & & & & & & & & & & II \\
\hline Astronium fraxinifolium & ASTFRAX6 & Urunde'y para & & & & & & & & & & I \\
\hline Atriplex eximia & ATREXIM6 & & I & III & & I & & & & & & \\
\hline Ayenia eliae & AYEELIA6 & & & & & & & & & & & II \\
\hline Ayenia odonellii & AYEODON6 & & & & II & & & & & I & I & \\
\hline Baccharis salicifolia & BACSALI6 & & & & I & & & & & & I & \\
\hline Baccharis trinervis & BACTRIN6 & & & & & & & & I & I & I & \\
\hline Basistemon spinosus & BASSPIN6 & & & & & & & & I & & I & I \\
\hline Bauhinia argentinensis & BAUARGE6 & Pata de buey & & & & & & & & & & II \\
\hline Boerhavia diffusa & BOEDIFF6 & & & & & & & & & & & $\mathrm{I}$ \\
\hline Borreria eryngioides & BORERYN6 & & & & & & & & & & & II \\
\hline Bougainvillea campanulata & BOUCAMP6 & Gallo espuela & & & & & & & I & I & I & \\
\hline Bromelia hieronymi & BROHIER6 & Karaguata & & I & I & & I & & II & II & I & \\
\hline Bromelia serra & BROSERR6 & Karaguata & & & I & I & I & I & III & IV & IV & \\
\hline Bulbostylis capillaris & BULCAPI6 & & & & & & & & & & & I \\
\hline Bulnesia bonariensis & BULBONA6 & & & & & & I & & I & & & \\
\hline Bulnesia sarmientoi & BULSARM6 & Palo santo & & & I & & I & & I & I & I & \\
\hline Capparicordis tweediana & CAPTWEE6 & Sacha membrillo & & II & III & I & $\mathrm{I}$ & & II & III & IV & \\
\hline Capsicum chacoënse & СAPCHAC6 & Ají silvestre & & & & & & & & I & I & \\
\hline Cardiospermum halicacabum & CARHALI6 & Kamuvu & & & & & & & & & I & I \\
\hline Carlowrightia sulcata & CARSULC6 & & & & & & & II & & & & \\
\hline Castela coccinea & CASCOCC6 & Mistol del zorro & & & I & & & I & I & I & II & I \\
\hline Ceiba chodatii & CEICHOD6 & Samu’u & & & & & I & & & I & I & \\
\hline Celtis ehrenbergiana & CELEHRE6 & Juasy’y, tala & & I & I & & & & I & II & II & I \\
\hline Centrosema virginianum & CENVIRG6 & & & & & & & & I & I & I & \\
\hline Cereus forbesii & CERFORB6 & Tuna & & I & & & & I & I & I & I & \\
\hline Chamaecrista arachiphylla & CHAARAC6 & & & & & & & & & & & II \\
\hline Chamaecrista serpens & CHASERP6 & & & & & & & & & & & III \\
\hline Cheilanthes obducta & CHEOBDU6 & & & & & & I & & I & & I & \\
\hline Chenopodium pilcomayense & CHEPILC6 & & I & I & & & & & & & & \\
\hline Chloris ciliata & CHLCILI6 & & & & & & & & & & & I \\
\hline Chloris elata & CHLELAT6 & & & & & & I & & & & & \\
\hline Cienfuegosia argentina & CIEARGE6 & & & & & & & & & & & I \\
\hline Cienfuegosia drummondii & CIEDRUM6 & $\begin{array}{l}\text { Planta del gallito de } \\
\text { agua, guaikuru ka'a }\end{array}$ & & I & & & & & & & & \\
\hline Cissus verticillata & CISVERT6 & Ka’avurã & & & & I & $\mathrm{I}$ & & I & & I & \\
\hline Cleistocactus baumannii & CLEBAUM6 & & & & & & II & & III & I & I & \\
\hline Cleome tucumanensis & CLETUCU6 & & & & & & & & & & & II \\
\hline Cnidoscolus albomaculatus & CNIALBO6 & & & & & & & & & & & IV \\
\hline Coccoloba spinescens & COCSPIN6 & & & & I & & & I & & & & \\
\hline Commelina erecta & COMEREC6 & & & & II & I & II & & II & I & II & \\
\hline Copernicia alba & COPALBA6 & Karanda’y & III & II & I & & & & & I & & \\
\hline Cordia glabrata & CORGLAB6 & Peterevy moroti & & & & & & & & & I & \\
\hline Croton bonplandianus & CROBONP6 & & $\mathrm{I}$ & II & $\mathrm{I}$ & & & I & & & & II \\
\hline Croton glandulosus & CROGLAN6 & & & & & & & & & & & II \\
\hline Croton gracilipes & CROGRAC6 & & & & & & & I & I & & II & \\
\hline Croton hieronymi & CROHIER6 & & & & & & & I & I & I & III & \\
\hline Croton orbignyanus & CORORBI6 & & & & & & & & & I & & \\
\hline Cucurbitella asperata & CUCASPE6 & & & & & & & & I & I & I & \\
\hline Cuscuta species & CUSSPEC6 & & & I & & & & & & & & \\
\hline Cyclolepis genistoides & CYCGENI6 & Palo azul & & & & & II & & I & I & & \\
\hline Cynodon dactylon & CYNDYCT6 & Pata de perdiz & I & I & & & & & & & & \\
\hline Cynodon niemfuensis & CYNNIEM6 & & II & I & I & & & & & & & \\
\hline Cynophalla retusa & CYNRETU6 & Indio kumanda & I & II & III & I & I & II & II & II & II & \\
\hline
\end{tabular}




\begin{tabular}{|c|c|c|c|c|c|c|c|c|c|c|c|c|}
\hline \multirow{2}{*}{ Arten } & \multirow{2}{*}{ Abkürzung } & \multirow{2}{*}{ Trivialname } & \multicolumn{10}{|c|}{ Gehölzgesellschaften } \\
\hline & & & 10 & 11 & 12 & 13 & 14 & 15 & 16 & 17 & 18 & 19 \\
\hline Cyperus aggregatus & CYPAGGR6 & & & & & & & & & & & I \\
\hline Cyperus corymbosus v. subnodosus & CYPCORY6 & & & & & I & & & & & & \\
\hline Cyperus species & CYPSPEC6 & & & & & & & I & & & & \\
\hline Deinacanthon urbanianum & DEIURBA6 & & & & I & I & $\mathrm{V}$ & IV & $\mathrm{V}$ & IV & III & \\
\hline Desmanthus virgatus & DESVIRG6 & & & I & II & & & & & & & I \\
\hline Desmodium incanum & DESINCA6 & & & & & & & & & & & I \\
\hline Deuterocohnia carmineoviridiflora & DEUCARM6 & & & & & & & & I & I & & \\
\hline Dicliptera squarrosa & DICSQUA6 & & & & & & & & & & I & I \\
\hline Digitaria insularis & DIGINSU6 & Kapi’i pororo & & & & & & & & & I & \\
\hline Digitaria species & DIGSPEC6 & & & & & & & & & & & I \\
\hline Diplokeleba floribunda & DIPFLOR6 & Palo piedra & & & & & & & & I & I & \\
\hline Ditaxis montevidensis & DITMONT6 & & & & I & & & & I & I & III & \\
\hline Dolichandra uncata & DOLUNCA6 & Uña de gato & & & & & & & & & I & \\
\hline Dolichandra unguiscati & DOLUNGU6 & Uña de gato & & & & & & & & & I & \\
\hline Dorstenia brasiliensis & DORBRAS6 & & & & & & & & I & & I & \\
\hline Dyckia ferox & DYCFERO6 & & & & & I & I & & & I & & \\
\hline Dyckia ragonesei & DYCRAGO6 & & & & & & I & & I & & I & \\
\hline Dyschoriste venturii & DYSVENT6 & & & & & & & & & & & I \\
\hline Echinopsis rhodotricha & ECHRHOD6 & & & & & I & II & I & II & & & \\
\hline Eclipta prostrata & ECLPROS6 & & I & & & & & & & & & \\
\hline Elionurus muticus & ELIMUTI6 & Espartillo & & & & & & & & & & III \\
\hline Eragrostis orthoclada & ERAORTH6 & & & & & & & & & & & I \\
\hline Erythroxylum cuneifolium & ERYCUNE6 & Coca del campo & & & & & & & & & I & \\
\hline Eupatorium catarium & EUPCATA6 & & & & & & & & & & & I \\
\hline Eupatorium christieanum & EUPCHRI6 & & & & & & & & & & I & \\
\hline Eupatorium patens & EUPPATE6 & & & & & & & & & & & I \\
\hline Euphorbia lasiocarpa & EUPLASI6 & & I & IV & I & II & II & II & I & I & I & I \\
\hline Euphorbia serpens & EUPSERP6 & tupasy kamby & III & & & & & & & & & \\
\hline Evolvulus convolvuloides & EVOCONV6 & & & I & III & & & & & & & \\
\hline Evolvulus sericeus & EVOSERI6 & & & & & & & & & & & II \\
\hline Fridericia dichotoma & FRIDICH6 & & & & I & & & & & I & II & II \\
\hline Fridericia truncata & FRIDTRUN6 & & & & & & & & I & I & II & \\
\hline Funastrum clausum & FUNCLAU6 & & III & I & I & & & & & & & \\
\hline Galactia latisiliqua & GALLATI6 & & & & I & & & & & & & \\
\hline Galactia longifolia & GALLONG6 & & & & II & & & II & & & & \\
\hline Gaya tarijensis & GAYTARI6 & & & & & & & & & & & I \\
\hline Gibasis geniculata & GIBGENI6 & & & I & & I & & & & & & \\
\hline Glinus radiatus & GLIRADI6 & & I & I & & & & & & & & \\
\hline Gomphrena perennis & GOMPERE6 & & & & & & & & & & I & III \\
\hline Gouinia paraguayensis & GOUPARA6 & & & & & I & I & I & II & III & I & \\
\hline Grindelia pulchella & GRIPULC6 & & & I & & I & & & & & & \\
\hline Gymnocalycium mihanovichii & GYMMIHA6 & & & & & & & & I & I & I & \\
\hline Harrisia bonplandii & HARBONP6 & & & & I & II & II & IV & I & II & II & \\
\hline Heliotropium campestre & HELCAMP6 & & & & & & & & & & & I \\
\hline $\begin{array}{l}\text { Heliotropium curassavicum } v . \\
\text { argentinum }\end{array}$ & HELCURA6 & & IV & III & & & & & I & & & \\
\hline Heliotropium dunaense & HELDUNA6 & & & & & & & & & & & I \\
\hline Heliotropium pallescens & HELPALL6 & & & & I & & & & & & & \\
\hline Herissantia crispa & HERCRIP6 & & & & & & & & & & & I \\
\hline Herreria montevidensis & HERMONT6 & Zarzaparrilla & & & & & & & I & I & II & \\
\hline Heterostachys ritteriana & HETRITT6 & & & & & & III & & I & & & \\
\hline Holmbergia tweedii & HOLTWEE6 & & & I & I & I & I & & I & & I & \\
\hline Ipomoea amnicola & IPOAMN6 & & & & & I & II & I & I & II & II & \\
\hline Ipomoea bonariensis & IPOBONA6 & & & & & & & I & & I & & \\
\hline Ipomoea grandifolia & IPOGRAN6 & & & & & & & & & I & & \\
\hline
\end{tabular}




\begin{tabular}{|c|c|c|c|c|c|c|c|c|c|c|c|c|}
\hline \multirow{2}{*}{ Arten } & \multirow{2}{*}{ Abkürzung } & \multirow{2}{*}{ Trivialname } & \multicolumn{10}{|c|}{ Gehölzgesellschaften } \\
\hline & & & 10 & 11 & 12 & 13 & 14 & 15 & 16 & 17 & 18 & 19 \\
\hline Jacaratia corumbensis & JACCORU6 & Yvy’a & & & & & & & & I & & \\
\hline Jacquemontia decumbens & JACDECU6 & & & & & & & & & & & II \\
\hline Janusia guaranitica & JANGUAR6 & & & & & & & & & & I & I \\
\hline Jatropha excisa & JATEXCI6 & & & & & & & & & & & I \\
\hline Jatropha grossidentata & JATGROSS6 & & & & & & & & & I & & \\
\hline Jatropha hieronymi & JATHIER6 & & & & & & & & & I & & \\
\hline Justicia corumbensis & JUSCORU6 & & & & & & & & & & I & \\
\hline Justicia dumetorum & JUSDUME6 & & & & I & & & II & & & I & \\
\hline Justicia squarrosa & JUSSQUA6 & & & & & & & & & & I & \\
\hline Justicia xylosteoides & JUSXYLO6 & & & & & & I & & II & II & I & \\
\hline Lantana balansae & LANBALA6 & & & & & & & & & I & I & \\
\hline Lasiacis sorghoidea & LASSORG6 & & & & & & & & & & I & \\
\hline Leptochloa fusca subsp. uninervia & LEPFUUN6 & & III & I & I & I & I & & I & & & \\
\hline Leptochloa virgata & LEPVIRG6 & & & & & & & & & & I & \\
\hline Lippia alba & LIPALBA6 & & & & & & & & I & & & \\
\hline Lophocarpinia aculeatifolia & LOPACUL6 & Hueso de sapo & & & & I & & & I & I & & \\
\hline Lycium americanum & LYCAMER6 & & II & II & II & IV & III & & III & I & & I \\
\hline Lycium boerhaaviaefolium & LYCBOER6 & Tala salada & & I & & & & & I & & & I \\
\hline Lycium cuneatum & LYCCUNE6 & & & & & & & & & & I & \\
\hline Lycium nodosum & LYCNODO6 & & II & II & III & I & & & & & & \\
\hline Macroptilium bracteatum & MACBRAC6 & & & & & & & & & & & I \\
\hline Macroptilium lathyroides & MACLATH6 & & & & & & & & & I & & I \\
\hline Malvastrum coromandelianum & MALCORO6 & & & & & & & & & & & I \\
\hline Mandevilla angustifolia & MANANGU6 & & & & & & & & & I & & \\
\hline Manihot guaranitica & MANGUAR6 & & & & & & & & I & I & I & \\
\hline Marsdenia castillonii & MARCAST6 & & & & & & & II & I & I & I & \\
\hline Mascagnia brevifolia & MASBREV6 & & & & I & & & & & & I & \\
\hline Maytenus vitis-idaea & MAYVITI6 & Sal de indio & & II & I & II & III & II & III & II & I & \\
\hline Megathyrsus maximus & MEGMAXI6 & & I & & & & & I & I & I & II & II \\
\hline Melochia ministella & MELMINI6 & & & & I & & & & & & & \\
\hline Melochia parvifolia & MELPARV6 & & & & I & & & & & & & \\
\hline Melochia werdermannii & MELWERD6 & & & & & & & & & & & III \\
\hline Microstachys hispida & MICHISP6 & & & & & & & & & & & II \\
\hline Mimosa debilis & MIMDEBI6 & & & & & & & & & & & III \\
\hline Mimosa detinens & MIMDETI6 & Jukeri & & & I & & & & I & & & \\
\hline Mimosa xanthocentra & MIMXANT6 & & & & & & & & & & & I \\
\hline Monvillea cavendischii & MONCAVE6 & & & I & I & & II & II & IV & II & III & \\
\hline Monvillea kroenleini & MONKROE6 & & & & & & & & & & I & \\
\hline Monvillea spegazzinii & MONSPEG6 & & & & & & I & & I & II & I & \\
\hline Neptunia pubescens & NEPPUBE6 & & III & & & & & & & & & \\
\hline Noticastrum acuminatum & NOTACUM6 & & & & & & & & & & & I \\
\hline Opuntia anacantha v. retrorsa & OPURETR6 & & & II & I & II & II & IV & III & I & I & \\
\hline Opuntia colubrina & OPUCOLU6 & & & & & & & & II & I & I & \\
\hline Opuntia elata var. cardiosperma & OPUCARD6 & & & & I & II & IV & & II & I & & \\
\hline Oxalis frutescens & OXAFRUT6 & & & & & & & & I & I & I & \\
\hline Oxypetalum arnottianum & OXYARNO6 & & & & & & & & & & & I \\
\hline Pappophorum krapovickasii & PAPKRAP6 & & & & & I & & & & & & IV \\
\hline Parkinsonia aculeata & PARACUL6 & Cina cina & IV & I & & & & & & & & \\
\hline Passiflora foetida & PASFOET6 & Mburukuja & & & & & I & & & I & & I \\
\hline Pavonia hieronymi & PAVHIER6 & & & & & & & & & & & I \\
\hline Pavonia vannii & PAVVANN6 & & & & & & & & & & & I \\
\hline Phyllostylon rhamnoides & PHYRHAM6 & Palo lanza & & & I & & & & & & III & \\
\hline Piptadeniopsis lomentifera & PIPLOME6 & & & & & & & & II & I & & I \\
\hline Pisonia zapallo & PISZAPA6 & & & & & & & & & & I & \\
\hline Polycarpon suffruticosum & POLSUFF6 & & & & I & & & I & & & & \\
\hline
\end{tabular}




\begin{tabular}{|c|c|c|c|c|c|c|c|c|c|c|c|c|}
\hline \multirow{2}{*}{ Arten } & \multirow{2}{*}{ Abkürzung } & \multirow{2}{*}{ Trivialname } & \multicolumn{10}{|c|}{ Gehölzgesellschaften } \\
\hline & & & 10 & 11 & 12 & 13 & 14 & 15 & 16 & 17 & 18 & 19 \\
\hline Porophyllum ruderale & PORRUDE6 & & & & & & & & & & & II \\
\hline Portulaca cryptopetala & PORCRYP6 & & I & I & I & & & & & & & $\mathrm{I}$ \\
\hline Portulaca grandiflora & PORGRAN6 & & I & & I & & & & & & & \\
\hline Portulaca oleracea & POROLER6 & & I & I & I & I & & & & & & I \\
\hline Portulaca species & PORSPEC6 & & & & & & I & & & & & I \\
\hline Priva boliviana & PRIBOLI6 & & & & II & & & & I & I & I & \\
\hline Prosopanche americana & PROAMER6 & & & & $\mathrm{I}$ & I & & & & I & & \\
\hline Prosopis hassleri & PROHASSL6 & & & & I & & & & & & & I \\
\hline Prosopis ruscifolia & PRORUSC6 & Viñal & III & IV & III & II & I & & I & I & & I \\
\hline Prosopis sericantha & PROSERI6 & & & & & & & & & I & & \\
\hline Pseudabutilon callimorphum & PSECALLI6 & & & & & & & & & I & I & \\
\hline Pseudabutilon virgatum & PSEVIRG6 & & & & & & & & & & & I \\
\hline Pterocaulon purpurascens & PTEPURP6 & Vira-vira poty & & I & & I & & & & & & \\
\hline Pterocaulon virgatum & PTEVIRG6 & & & & & & & & & & & I \\
\hline Pteroglossa lurida & PTELURI6 & & & & & & & & & I & & \\
\hline Quiabentia verticillata & QUIVERT6 & & & & & & I & & & & & \\
\hline Rhynchosia burkartii & RHYBURK6 & & & & & & & & & & & IV \\
\hline Rivina humilis & RIVHUMI6 & & & & I & & & & & I & II & \\
\hline Ruellia erythropus & RUEERYT6 & & & & & & & & I & I & III & \\
\hline Ruellia hygrophila & RUEHYGR6 & & & & & & & III & & I & II & \\
\hline Ruellia simplex & RUESIMP6 & & I & III & $\mathrm{V}$ & II & II & IV & I & IV & I & \\
\hline Salta triflora & SALTRIF6 & Guaimi pire & & & & & & I & II & II & II & \\
\hline Salvia cardiophylla & SALCARD6 & & & & & & & & & & & I \\
\hline Sarcocornia ambigua & SARAMB6 & & III & IV & I & III & II & & I & & & \\
\hline Sarcotoxicum salicifolium & SARSALI6 & Sacha sandía & & & & & I & & I & I & I & \\
\hline Schaefferia argentinensis & SCHARGE6 & & & & & & & & & I & I & \\
\hline Schinopsis cornuta & SCHCORN6 & Urunde'y boli & & & & & & & & & & II \\
\hline Schizachyrium sp. & SCHSPEC6 & Pasto rojo & & & & & & & & & & I \\
\hline Schubertia grandiflora & SCHGRAN6 & & & & & & & & & & I & \\
\hline Schwenckia americana & SCHAMER6 & & & & & & & & & & & I \\
\hline Sclerophylax spinescens & SCLSPIN6 & & & I & & I & I & & & & & \\
\hline Scoparia dulcis & SCODULC6 & & & & & & & & & & & III \\
\hline Selaginella sellowii & SELSELL6 & & & & & & & & I & & & I \\
\hline Senna morongii & SENMORO6 & & & & I & & & & & & & \\
\hline Senna pendula & SENPEND6 & Taperyvaimi & & & & & & & & I & & \\
\hline Serjania marginata & SERMARG6 & & & & I & & & & & & I & \\
\hline Serjania species & SERSPEC6 & & & & & & & & & I & I & \\
\hline Sesbania virgata & SESVIRG6 & Palo negro & II & & & & & & & & & \\
\hline Sesuvium portulacastrum & SESPORT6 & & III & IV & & $\mathrm{V}$ & II & & I & & & \\
\hline Setaria geminata & SETGEMI6 & & II & I & & & & & & & & \\
\hline Setaria nicorae & SETNICO6 & & & I & $\mathrm{V}$ & I & II & $\mathrm{V}$ & IV & $\mathrm{V}$ & IV & II \\
\hline Sida anomala & SIDANOM6 & & & I & $\mathrm{I}$ & & & I & & & & I \\
\hline Sida cabreriana & SIDCABR6 & & & & & & & I & & I & & \\
\hline Sida dictyocarpa & SIDDICT6 & & & & I & & & I & II & I & III & \\
\hline Sideroxylon obtusifolium & SIDOBTU6 & Guajayvi ra’i & & & & & & & & I & I & \\
\hline Solanum aridum & SOLARID6 & & & & I & & I & & I & I & I & \\
\hline Solanum elaeagnifolium & SOLELAE6 & & & I & & I & & & & & & \\
\hline Solanum glaucophyllum & SOLGLAU6 & & II & I & & & & & & & & \\
\hline Solanum multispinum & SOLMULT6 & & I & & & & & & & & & \\
\hline Solanum sisymbriifolium & SOLSISY6 & & I & & & & & & & & & \\
\hline Spathicarpa hastifolia & SPAHAST6 & ka’a-ite & & & & & & & & & I & \\
\hline Spergula ramosa & SPERAMO6 & & & I & & & & & & & & \\
\hline Spilanthes paraguayensis & SPIPARA6 & & & & I & & & & & & & \\
\hline Sporobolus pyramidatus & SPOPYRA6 & & IV & $\mathrm{V}$ & & III & II & & & I & & \\
\hline Staelia virgata & STAVIRG6 & & & & & & & & & & I & I \\
\hline
\end{tabular}




\begin{tabular}{|c|c|c|c|c|c|c|c|c|c|c|c|c|}
\hline \multirow{2}{*}{ Arten } & \multirow{2}{*}{ Abkürzung } & \multirow{2}{*}{ Trivialname } & \multicolumn{10}{|c|}{ Gehölzgesellschaften } \\
\hline & & & 10 & 11 & 12 & 13 & 14 & 15 & 16 & 17 & 18 & 19 \\
\hline Stemodia ericifolia & STEERIC6 & & $\mathrm{I}$ & II & $\mathrm{I}$ & & & & & & & \\
\hline Stetsonia coryne & STECORY6 & Cardón, tuna & & & & & & & I & & & \\
\hline Tabebuia aurea & TABAURE6 & Paratodo & & & & & & & & & & II \\
\hline Tabebuia nodosa & TABNODO6 & Labón, palo cruz & & I & II & $\mathrm{I}$ & & II & & I & I & \\
\hline Talinum fruticosum & TALFRUT6 & & & & & & I & & I & I & I & I \\
\hline Talinum paniculatum & TALPANI6 & & I & I & I & II & II & & I & I & & \\
\hline Talinum polygaloides & TALPOLY6 & & & & & & & & & I & & \\
\hline Tillandsia mereliana & TILMERE6 & & & & & $\mathrm{I}$ & IV & & I & I & & \\
\hline Tournefortia rubicunda & TOURUBI6 & & & & & & & & & & I & \\
\hline Tragia hieronymii & TRAHIER6 & & & & & & & & I & I & III & I \\
\hline Trianthema portulacastrum & TRIPORT6 & & & $\mathrm{I}$ & & $\mathrm{I}$ & & & & & & \\
\hline Trichloris crinita & TRICRIN6 & & & $\mathrm{I}$ & II & II & III & & III & I & & I \\
\hline Trithrinax schyzophylla & TRISCHY6 & Karandilla & & & & & II & II & IV & $\mathrm{V}$ & $\mathrm{V}$ & \\
\hline Trixis divaricata & TRIDIVA6 & & & & & & & & & & I & \\
\hline Turnera grandiflora & TURGRAN6 & & & & & & & & & & & I \\
\hline Turnera krapovickasii & TURKRAP6 & & & & & & & & & & & II \\
\hline Turnera weddelliana & TURWEDD6 & & & & & & & & & & I & \\
\hline Urochloa adspersa & UROADSP6 & & & $\mathrm{I}$ & $\mathrm{I}$ & & I & & I & I & I & \\
\hline Urochloa paucispicata & UROPAUC6 & & & & & & & & & & & III \\
\hline Vernonia niederleinii & VERNIED6 & & & & & & & & & & & I \\
\hline Vigna luteola & VIGLUTE6 & & & & I & & & II & & & & \\
\hline Waltheria communis & WALCUMM6 & & & & & & & & & & I & I \\
\hline Waltheria indica & WALINDI6 & & & & & & & & & & & I \\
\hline Wedelia species & WEDSPEC6 & & & & & & & & & & & I \\
\hline Wissadula densiflora & WISDENS6 & & & & III & & I & II & II & II & II & \\
\hline Ximenia americana & XIMAMER6 & $\begin{array}{l}\text { Pata de monte, indio } \\
\text { kurupa'y }\end{array}$ & & & & & & & & I & I & I \\
\hline Zanthoxylum fagara & ZANFAGA6 & Mbarakajá pyapê & & & & & & & & & I & \\
\hline Zexmenia brachylepis & ZEXBRAC6 & & & & & & & & I & & I & II \\
\hline Ziziphus mistol & ZIZMIST6 & Mistol & I & & II & $\mathrm{I}$ & II & I & II & III & I & I \\
\hline Zornia latifolia & ZORLATI6 & & & & & & & & & & & II \\
\hline EPIPHYTEN & & & & & & & & & & & & \\
\hline Microgramma vacciniifolia & MICVACC7 & Anguja ruguái & & & & & & & I & I & II & \\
\hline Phoradendron argentinum & PSICORD7 & Ka’avó tyre’y & & II & $\mathrm{I}$ & $\mathrm{I}$ & & & & & I & \\
\hline Psittacanthus cordatus & PHOARGE7 & & & $\mathrm{I}$ & & & & & & & & \\
\hline Rhipsalis baccifera & RHIBACC7 & Suelta con suelta & & & & & & & & & I & \\
\hline Selenicereus setaceus & SELSETA7 & & & & & & & II & I & I & I & \\
\hline Tillandsia bandensis & TILBAND7 & Clavel del aire & & I & & $\mathrm{I}$ & I & I & I & I & I & \\
\hline Tillandsia duratii v. saxatilis & TILDURA7 & Clavel del aire & & $\mathrm{I}$ & II & II & $\mathrm{V}$ & II & III & $\mathrm{V}$ & III & I \\
\hline Tillandsia funebris & TILFUNE7 & Clavel del aire & & & & $\mathrm{I}$ & I & I & I & II & I & $\mathrm{I}$ \\
\hline Tillandsia loliacea & TILLOLI7 & Clavel del aire & & & $\mathrm{I}$ & II & III & IV & $\mathrm{V}$ & IV & IV & \\
\hline Tillandsia mereliana & TILMERE7 & & & & & & II & & I & I & & \\
\hline Tillandsia minutiflora & TILMINU7 & Clavel del aire & & & & & I & & & I & I & \\
\hline Tillandsia recurvifolia & TILRECU7 & Clavel del aire & & I & & $\mathrm{I}$ & I & IV & II & II & II & \\
\hline Tillandsia reichenbachii & TILREICH7 & Clavel del aire & & & $\mathrm{I}$ & $\mathrm{I}$ & & I & III & I & II & \\
\hline Tillandsia vernicosa & TILVERN7 & Clavel del aire & & & & & I & & I & I & I & \\
\hline
\end{tabular}




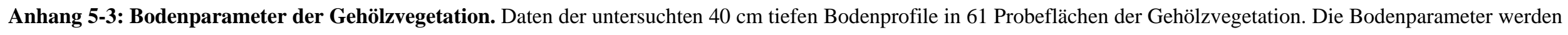

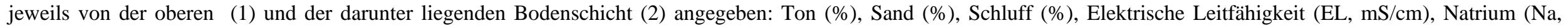
$\mathrm{cmol}_{\mathrm{c}} / \mathrm{kg}$ ), $\mathrm{pH}$, Organische Substanz (Org, \%), Calcium (Ca, $\mathrm{cmol}_{\mathrm{c}} / \mathrm{kg}$ ) und Magnesium $\left(\mathrm{Mg}, \mathrm{cmol}_{\mathrm{c}} / \mathrm{kg}\right.$ ).

\begin{tabular}{|c|c|c|c|c|c|c|c|c|c|c|c|c|c|c|c|c|c|c|c|}
\hline Einheit & Probefl. & Ton1 & Ton2 & Sand1 & Sand2 & Schluff1 & Schluff2 & EL1 & EL2 & Na1 & $\mathrm{Na} 2$ & pH1 & pH2 & Org1 & Org2 & Ca1 & $\mathrm{Ca} 2$ & Mg1 & Mg2 \\
\hline 10 & 94 & 31.00 & 8.90 & 60.00 & 84.65 & 11.00 & 4.20 & 0.06 & 1.71 & 6.57 & 6.44 & 4.25 & 5.99 & 3.03 & 0.88 & 6.75 & 7.57 & 7.91 & 6.68 \\
\hline 10 & 166 & 40.00 & 40.00 & 42.00 & 42.00 & 18.00 & 18.00 & 1.25 & 1.25 & 1.26 & 1.26 & 6.50 & 6.50 & 1.86 & 1.86 & 5.92 & 5.92 & 1.91 & 1.91 \\
\hline 10 & 208 & 23.40 & 30.00 & 68.10 & 60.00 & 8.50 & 10.00 & 0.43 & 0.53 & 3.34 & 4.36 & 5.72 & 5.93 & 2.10 & 0.84 & 10.17 & 8.69 & 7.80 & 8.08 \\
\hline 11 & 96 & 43.00 & 43.00 & 47.00 & 47.00 & 18.00 & 18.00 & 2.92 & 2.92 & 30.55 & 30.55 & 7.72 & 7.72 & 0.86 & 0.86 & 4.82 & 4.82 & 9.34 & 9.34 \\
\hline 11 & 140 & 41.70 & 40.00 & 14.15 & 15.00 & 44.15 & 45.00 & 3.20 & 6.00 & 8.68 & 25.83 & 5.30 & 6.25 & 4.45 & 0.69 & 2.97 & 1.87 & 1.90 & 1.87 \\
\hline 11 & 209 & 30.00 & 30.00 & 62.00 & 62.00 & 8.00 & 8.00 & 4.50 & 4.50 & 10.49 & 10.49 & 7.02 & 7.02 & 2.72 & 2.72 & 15.60 & 15.60 & 9.24 & 9.24 \\
\hline 12 & 1 & 33.48 & 36.48 & 51.02 & 49.52 & 15.50 & 14.00 & 4.03 & 5.33 & 4.57 & 5.78 & 7.19 & 7.56 & 1.85 & 0.77 & 1.74 & 1.39 & 1.61 & 1.69 \\
\hline 12 & 2 & 29.68 & 34.48 & 52.32 & 53.52 & 18.00 & 12.00 & 1.37 & 3.17 & 1.80 & 2.50 & 6.94 & 7.33 & 2.64 & 0.77 & 4.52 & 2.38 & 3.67 & 3.53 \\
\hline 12 & 168 & 24.60 & 15.00 & 35.40 & 31.00 & 40.00 & 54.00 & 0.99 & 1.29 & 4.88 & 4.68 & 4.52 & 4.75 & 4.32 & 2.87 & 2.84 & 2.76 & 1.89 & 1.91 \\
\hline 12 & 172 & 15.00 & 15.00 & 32.00 & 32.00 & 53.00 & 53.00 & 2.10 & 2.10 & 4.68 & 4.68 & 5.01 & 5.01 & 4.25 & 4.25 & 4.54 & 4.54 & 48.93 & 48.93 \\
\hline 12 & 206 & 6.00 & 6.00 & 86.00 & 86.00 & 8.00 & 8.00 & 0.33 & 0.33 & 2.10 & 2.10 & 6.10 & 6.10 & 1.80 & 1.80 & 7.70 & 7.70 & 7.38 & 7.38 \\
\hline 13 & 97 & 32.00 & 17.70 & 61.00 & 76.95 & 8.00 & 5.80 & 3.04 & 3.60 & 25.20 & 22.68 & 8.44 & 8.56 & 1.04 & 0.64 & 24.12 & 16.16 & 10.06 & 7.69 \\
\hline 13 & 116 & 11.00 & 11.00 & 80.00 & 80.00 & 9.00 & 9.00 & 4.80 & 4.80 & 20.83 & 20.83 & 8.28 & 8.28 & 1.44 & 1.44 & 2.04 & 2.04 & 7.03 & 7.03 \\
\hline 13 & 117 & 5.50 & 6.00 & 90.50 & 90.00 & 4.00 & 4.00 & 3.40 & 4.90 & 15.20 & 25.46 & 7.94 & 7.50 & 1.20 & 0.49 & 4.61 & 2.04 & 4.77 & 4.99 \\
\hline 13 & 201 & 40.00 & 40.00 & 41.00 & 41.00 & 19.00 & 19.00 & 5.60 & 5.60 & 34.70 & 34.70 & 7.07 & 7.07 & 0.69 & 0.69 & 9.68 & 9.68 & 8.08 & 8.08 \\
\hline 14 & 11 & 18.48 & 18.48 & 54.52 & 53.52 & 22.00 & 26.00 & 2.44 & 4.67 & 7.25 & 12.50 & 7.37 & 7.99 & 2.97 & 1.03 & 12.28 & 17.43 & 25.41 & 42.35 \\
\hline 14 & 62 & 31.60 & 32.00 & 59.80 & 60.00 & 8.60 & 8.00 & 7.94 & 7.90 & 29.42 & 33.21 & 8.34 & 8.51 & 1.49 & 0.49 & 24.75 & 24.37 & 9.57 & 9.43 \\
\hline 14 & 84 & 8.60 & 11.00 & 72.00 & 60.00 & 7.40 & 9.00 & 1.59 & 2.36 & 27.99 & 29.26 & 7.34 & 8.48 & 1.77 & 0.39 & 8.88 & 7.37 & 8.33 & 9.89 \\
\hline 14 & 137 & 11.00 & 25.25 & 80.00 & 66.50 & 9.00 & 9.75 & 4.00 & 5.20 & 2.93 & 22.72 & 8.61 & 7.55 & 4.40 & 1.63 & 12.25 & 11.49 & 9.30 & 8.28 \\
\hline 14 & 159 & 24.00 & 15.00 & 48.40 & 31.00 & 27.60 & 54.00 & 3.96 & 6.90 & 10.78 & 20.18 & 6.64 & 7.08 & 4.04 & 1.18 & 7.58 & 8.29 & 1.96 & 1.96 \\
\hline 15 & 4 & 30.48 & 32.48 & 53.02 & 51.52 & 16.50 & 16.00 & 4.61 & 5.96 & 4.77 & 6.09 & 6.41 & 6.96 & 1.99 & 0.58 & 3.96 & 4.36 & 2.46 & 2.02 \\
\hline 15 & 5 & 27.98 & 26.48 & 52.52 & 53.52 & 19.50 & 20.00 & 1.22 & 1.46 & 8.37 & 10.94 & 6.30 & 6.61 & 2.09 & 0.74 & 3.47 & 3.37 & 2.58 & 2.07 \\
\hline 15 & 6 & 25.98 & 30.48 & 50.52 & 47.52 & 23.50 & 22.00 & 1.31 & 1.96 & 2.38 & 5.94 & 6.74 & 7.33 & 3.61 & 1.54 & 8.37 & 8.51 & 4.41 & 4.24 \\
\hline 15 & 7 & 31.98 & 34.48 & 49.02 & 47.52 & 19.00 & 18.00 & 0.43 & 0.51 & 10.84 & 14.06 & 6.80 & 7.24 & 2.11 & 0.71 & 7.57 & 8.51 & 3.70 & 3.76 \\
\hline 15 & 8 & 31.48 & 32.48 & 50.02 & 49.52 & 18.50 & 18.00 & 10.21 & 11.58 & 4.22 & 5.31 & 6.20 & 6.44 & 2.23 & 1.96 & 4.06 & 4.36 & 2.55 & 2.07 \\
\hline 15 & 9 & 32.48 & 34.48 & 49.02 & 47.52 & 18.50 & 18.00 & 5.43 & 7.13 & 10.60 & 13.75 & 7.54 & 8.00 & 1.60 & 0.42 & 6.24 & 6.73 & 3.88 & 3.86 \\
\hline 15 & 10 & 33.48 & 36.48 & 43.52 & 41.52 & 23.00 & 22.00 & 5.08 & 6.62 & 10.22 & 13.13 & 7.16 & 7.71 & 1.74 & 0.29 & 6.43 & 6.73 & 4.28 & 4.42 \\
\hline 15 & 17 & 28.48 & 32.48 & 46.52 & 43.52 & 25.00 & 24.00 & 0.52 & 0.94 & 3.91 & 6.25 & 6.30 & 7.14 & 3.61 & 2.28 & 5.15 & 5.15 & 6.07 & 8.00 \\
\hline 15 & 179 & 40.00 & 40.00 & 16.00 & 16.00 & 44.00 & 44.00 & 2.70 & 2.70 & 16.95 & 16.95 & 5.69 & 5.69 & 4.16 & 4.16 & 18.56 & 18.56 & 6.21 & 6.21 \\
\hline 16 & 63 & 12.00 & 12.00 & 79.30 & 79.00 & 8.70 & 9.00 & 0.42 & 0.55 & 3.75 & 5.22 & 5.07 & 5.06 & 1.78 & 1.27 & 5.20 & 5.48 & 4.00 & 4.83 \\
\hline 16 & 142 & 40.00 & 40.00 & 16.00 & 16.00 & 44.00 & 44.00 & 1.02 & 1.02 & 4.52 & 4.52 & 6.45 & 6.45 & 4.87 & 4.87 & 4.15 & 4.15 & 1.87 & 1.87 \\
\hline 16 & 191 & 40.00 & 40.00 & 41.00 & 41.00 & 19.00 & 19.00 & 3.00 & 3.00 & 16.95 & 16.95 & 7.18 & 7.18 & 1.12 & 1.12 & 15.60 & 15.60 & 9.01 & 9.01 \\
\hline 16 & 197 & 5.00 & 5.00 & 90.00 & 90.00 & 5.00 & 5.00 & 5.40 & 5.40 & 26.63 & 26.63 & 7.48 & 7.48 & 1.09 & 1.09 & 6.71 & 6.71 & 7.61 & 7.61 \\
\hline
\end{tabular}




\begin{tabular}{|c|c|c|c|c|c|c|c|c|c|c|c|c|c|c|c|c|c|c|c|}
\hline Einheit & Probefl. & Ton1 & Ton2 & Sand1 & Sand2 & Schluff1 & Schluff2 & EL1 & EL2 & Na1 & $\mathrm{Na} 2$ & pH1 & pH2 & Org1 & Org2 & Ca1 & $\mathrm{Ca} 2$ & Mg1 & Mg2 \\
\hline 16 & 199 & 15.00 & 15.00 & 71.00 & 71.00 & 14.00 & 14.00 & 2.50 & 2.50 & 29.05 & 29.05 & 6.48 & 6.48 & 2.07 & 2.07 & 14.61 & 14.61 & 8.54 & 8.54 \\
\hline 17 & 3 & 30.48 & 36.48 & 52.52 & 49.52 & 17.00 & 14.00 & 1.85 & 3.52 & 10.86 & 15.30 & 6.36 & 7.51 & 2.60 & 0.90 & 2.18 & 1.58 & 2.80 & 2.07 \\
\hline 17 & 12 & 28.38 & 30.48 & 54.22 & 53.52 & 17.40 & 16.00 & 3.11 & 4.68 & 8.67 & 13.13 & 7.16 & 7.80 & 3.75 & 2.70 & 18.95 & 25.74 & 2.20 & 1.41 \\
\hline 17 & 13 & 31.98 & 34.48 & 52.52 & 51.52 & 15.50 & 14.00 & 0.04 & 0.00 & 8.73 & 11.56 & 6.51 & 6.97 & 2.92 & 2.05 & 12.87 & 15.05 & 7.22 & 8.47 \\
\hline 17 & 14 & 32.48 & 34.48 & 49.02 & 47.52 & 18.50 & 18.00 & 0.17 & 0.20 & 3.35 & 4.38 & 6.50 & 6.95 & 2.50 & 1.16 & 10.80 & 12.28 & 6.24 & 7.06 \\
\hline 17 & 15 & 24.48 & 24.48 & 49.52 & 49.52 & 24.20 & 26.00 & 2.88 & 4.09 & 5.78 & 8.13 & 6.29 & 6.88 & 3.67 & 2.54 & 7.96 & 8.32 & 3.94 & 4.14 \\
\hline 17 & 16 & 33.28 & 34.48 & 47.92 & 47.52 & 18.80 & 18.00 & 5.31 & 6.62 & 11.28 & 13.75 & 6.36 & 6.70 & 2.04 & 0.93 & 8.79 & 9.50 & 6.85 & 7.53 \\
\hline 17 & 18 & 29.48 & 30.48 & 47.52 & 47.52 & 23.00 & 22.00 & 0.21 & 0.21 & 6.33 & 7.19 & 6.36 & 6.50 & 2.53 & 2.38 & 4.26 & 4.36 & 7.88 & 8.00 \\
\hline 17 & 19 & 27.38 & 28.48 & 45.72 & 43.52 & 26.90 & 28.00 & 0.05 & 0.06 & 1.20 & 2.13 & 5.82 & 6.40 & 3.93 & 2.73 & 5.36 & 5.15 & 4.04 & 4.14 \\
\hline 17 & 78 & 43.00 & 43.00 & 46.00 & 46.00 & 12.25 & 19.00 & 1.94 & 6.60 & 7.16 & 25.30 & 5.86 & 7.80 & 3.58 & 0.58 & 13.51 & 20.59 & 9.43 & 10.12 \\
\hline 17 & 98 & 5.00 & 11.00 & 91.00 & 80.00 & 4.00 & 9.00 & 2.70 & 8.10 & 5.19 & 21.38 & 5.82 & 8.44 & 5.11 & 1.04 & 15.44 & 11.58 & 8.15 & 8.63 \\
\hline 17 & 99 & 7.15 & 4.00 & 87.05 & 92.00 & 5.80 & 4.00 & 2.66 & 4.38 & 2.73 & 4.58 & 6.39 & 7.48 & 3.93 & 1.94 & 28.94 & 37.63 & 6.17 & 6.71 \\
\hline 17 & 100 & 24.95 & 11.00 & 66.60 & 81.00 & 7.90 & 7.00 & 1.52 & 2.50 & 17.27 & 29.02 & 7.00 & 8.12 & 2.63 & 0.68 & 18.04 & 24.12 & 9.63 & 10.06 \\
\hline 17 & 120 & 21.00 & 31.00 & 70.50 & 61.00 & 9.50 & 10.00 & 2.85 & 5.50 & 12.66 & 24.69 & 5.05 & 5.19 & 3.49 & 1.22 & 16.85 & 25.53 & 7.37 & 9.52 \\
\hline 17 & 121 & 11.40 & 12.00 & 80.60 & 80.00 & 8.00 & 8.00 & 1.87 & 4.50 & 8.21 & 17.75 & 6.70 & 4.94 & 5.04 & 3.01 & 6.33 & 5.11 & 7.67 & 9.30 \\
\hline 17 & 138 & 11.00 & 11.00 & 79.65 & 79.00 & 9.35 & 10.00 & 3.10 & 5.70 & 10.24 & 22.38 & 7.66 & 5.88 & 5.15 & 2.86 & 22.41 & 33.69 & 8.95 & 9.98 \\
\hline 17 & 141 & 40.00 & 40.00 & 15.00 & 15.00 & 45.00 & 45.00 & 4.30 & 4.30 & 6.30 & 6.30 & 7.28 & 7.28 & 3.70 & 3.70 & 7.90 & 7.90 & 1.91 & 1.91 \\
\hline 17 & 160 & 12.80 & 40.00 & 76.80 & 40.00 & 10.40 & 20.00 & 2.58 & 6.10 & 5.55 & 12.91 & 5.91 & 7.82 & 5.42 & 1.15 & 29.30 & 113.36 & 1.56 & 0.14 \\
\hline 17 & 210 & 29.00 & 29.00 & 63.00 & 63.00 & 8.00 & 8.00 & 0.92 & 0.92 & 6.13 & 6.13 & 6.72 & 6.72 & 2.69 & 2.69 & 16.59 & 16.59 & 8.78 & 8.78 \\
\hline 18 & 82 & 5.00 & 5.00 & 91.00 & 91.00 & 4.00 & 4.00 & 0.04 & 0.06 & 0.98 & 2.21 & 5.00 & 4.87 & 1.43 & 0.79 & 4.91 & 5.48 & 2.99 & 4.37 \\
\hline 18 & 101 & 43.50 & 44.00 & 46.00 & 46.00 & 18.50 & 18.00 & 1.10 & 2.16 & 3.51 & 6.57 & 6.14 & 6.90 & 2.67 & 0.98 & 10.13 & 9.65 & 9.10 & 10.30 \\
\hline 18 & 103 & 11.00 & 11.00 & 79.00 & 79.00 & 8.00 & 8.00 & 0.20 & 0.20 & 0.61 & 0.61 & 4.75 & 4.75 & 4.57 & 4.57 & 5.79 & 5.79 & 4.07 & 4.07 \\
\hline 18 & 173 & 40.00 & 40.00 & 41.00 & 41.00 & 19.00 & 19.00 & 1.21 & 1.21 & 6.46 & 6.46 & 6.83 & 6.83 & 2.72 & 2.72 & 18.56 & 18.56 & 9.48 & 9.48 \\
\hline 18 & 198 & 5.00 & 5.00 & 87.00 & 87.00 & 8.00 & 8.00 & 2.20 & 2.20 & 15.33 & 15.33 & 5.98 & 5.98 & 3.36 & 3.36 & 22.51 & 22.51 & 8.78 & 8.78 \\
\hline 19 & 64 & 5.00 & 5.00 & 90.50 & 91.00 & 4.50 & 4.00 & 0.01 & 0.01 & 0.55 & 1.11 & 5.06 & 4.75 & 0.59 & 0.42 & 4.06 & 4.53 & 1.84 & 2.30 \\
\hline 19 & 81 & 5.00 & 5.00 & 90.65 & 90.00 & 4.35 & 5.00 & 0.45 & 1.28 & 1.42 & 3.48 & 4.67 & 4.61 & 0.26 & 0.18 & 2.65 & 2.65 & 1.09 & 1.84 \\
\hline 19 & 114 & 11.00 & 11.00 & 79.00 & 79.00 & 8.00 & 8.00 & 0.10 & 0.10 & 0.15 & 0.15 & 5.63 & 5.63 & 1.22 & 1.22 & 2.89 & 2.89 & 1.92 & 1.92 \\
\hline 19 & 134 & 4.00 & 4.00 & 92.00 & 92.00 & 4.00 & 4.00 & 0.12 & 0.12 & 1.08 & 1.08 & 6.83 & 6.83 & 0.77 & 0.77 & 2.04 & 2.04 & 4.76 & 4.76 \\
\hline
\end{tabular}




\section{Ordinationen}

Anhang 5-4: Log-file der Korrespondenzanalyse (DCA) der Gesellschaften 10 bis 16. Ermittlung der Gradientenlänge der Gesellschaften 10 bis 16

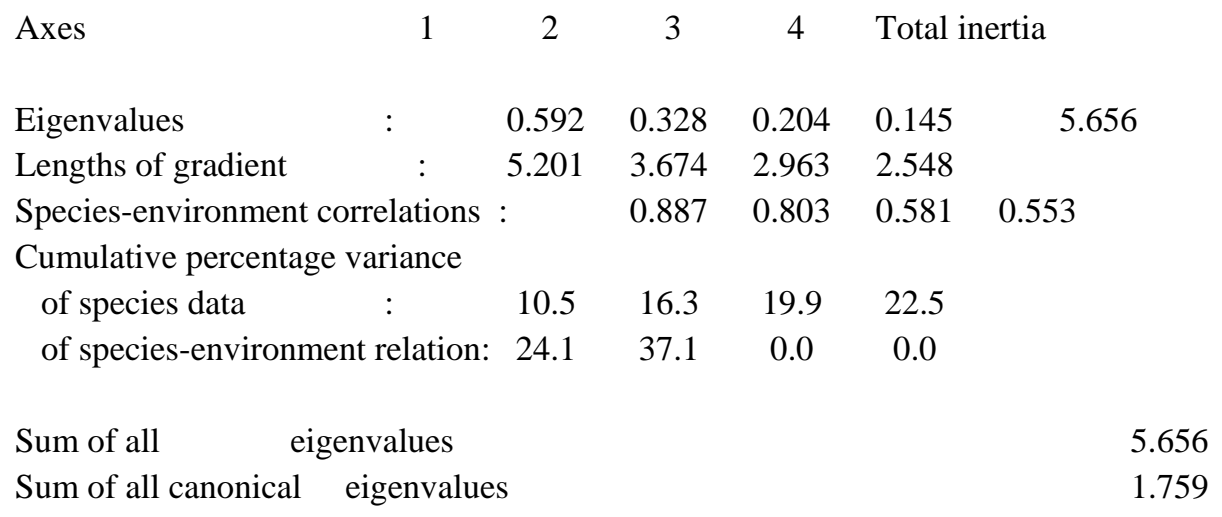

\section{Anhang 5-5: Log-file der Hauptgradientenanalyse (PCA) der Waldgesellschaften (Einheiten 17 und 18)}

Da die Korrespondenzanalyse (DCA) einen kurzen Gradienten auf der ersten Achse (2.082) aufwies, wurde für weitere Schritte eine Hauptgradientenanalyse gewählt.

\begin{tabular}{|c|c|c|c|c|c|}
\hline Axes & 2 & 3 & 4 & \multicolumn{2}{|c|}{ Total variance } \\
\hline Eigenvalues & 0.082 & 0.063 & 0.048 & 0.047 & 1.000 \\
\hline Species-environment correlations & : & 0.892 & 0.666 & 0.645 & 0.624 \\
\hline \multicolumn{6}{|l|}{ Cumulative percentage variance } \\
\hline of species data & 8.2 & 14.4 & 19.3 & 24.0 & \\
\hline of species-environment relation: & 18.9 & 26.9 & 32.8 & 38.1 & \\
\hline
\end{tabular}

$\begin{array}{ll}\text { Sum of all } \quad \text { eigenvalues } & 1.000\end{array}$

$\begin{array}{ll}\text { Sum of all canonical eigenvalues } & 0.344\end{array}$

\section{Anhang 5-6: Log-file der indirekte Gradientenanalyse (DCA) der Bodenparameter}

No. of active samples: 57

No. of active species: 233

\begin{tabular}{|c|c|c|c|c|c|c|}
\hline Axes & 1 & 2 & 3 & 4 & \multicolumn{2}{|c|}{ Total inertia } \\
\hline Eigenvalues & $:$ & 0.707 & 0.255 & 0.190 & 0.141 & 5.273 \\
\hline Lengths of gradient & : & 6.741 & 2.730 & 2.338 & 2.163 & \\
\hline Species-environment & elations & & 0.584 & 0.758 & 0.711 & 0.656 \\
\hline \multicolumn{7}{|c|}{ Cumulative percentage variance } \\
\hline of species data & & 13.4 & 18.2 & 21.8 & 24.5 & \\
\hline of species-environ & elatiol & 10.4 & 18.9 & 0.0 & 0.0 & \\
\hline
\end{tabular}

Sum of all eigenvalues $\quad 5.273$

$\begin{array}{ll}\text { Sum of all canonical eigenvalues } & 1.818\end{array}$ 
Anhang 5-7: Direkte Gradientenanalyse (CCA) der Bodenparameter. Analyse der 57 Probeflächen (Einheiten 10 bis 18) mit Bodenproben zur Ermittlung der signifikanten Bodenvariablen

\begin{tabular}{|c|c|c|c|c|c|}
\hline Axes & 2 & 3 & 4 & \multicolumn{2}{|c|}{ Total inertia } \\
\hline Eigenvalues & 0.326 & 0.169 & 0.114 & 0.088 & 5.273 \\
\hline Species-environment correlations & : & 0.881 & 0.856 & 0.813 & 0.691 \\
\hline \multicolumn{6}{|l|}{ Cumulative percentage variance } \\
\hline of species data & 6.2 & 9.4 & 11.6 & 13.2 & \\
\hline of species-environment relation: & 41.7 & 63.3 & 77.8 & 89.1 & \\
\hline
\end{tabular}

Sum of all eigenvalues $\quad 5.273$

$\begin{array}{ll}\text { Sum of all canonical eigenvalues } & 0.783\end{array}$

*** Unrestricted permutation ***

Seeds: 23239945

$\underline{\text { Manuelle Auswahl der Umweltvariablen mit einer Signifikanz } \leq 0.05}$

Na2: P-value 0.0020 (variable $10 ; \mathrm{F}$-ratio $=2.52$; number of permutations $=499$ )

Sand1: P-value 0.0120 (variable $3 ; \mathrm{F}$-ratio= 1.65; number of permutations $=499$ )

pH1: P-value 0.0280 (variable $11 ; \mathrm{F}$-ratio= 1.54; number of permutations $=499$ )

Schluff2: P-value 0.0500 (variable 6 ; F-ratio= 1.49; number of permutations $=499$ )

Org1: P-value 0.0340 (variable 13; F-ratio= 1.49; number of permutations $=499$ ) 